\title{
Risiken und Chancen des Paulownia-Anbaus unter mitteleuropäischen Bedingungen
}

\author{
Dissertation \\ zur Erlangung des Doktorgrades: Doctor forestalium (Dr. forest.) \\ der Fakultät für Forstwissenschaften und Waldökologie \\ der Georg-August-Universität Göttingen
}

vorgelegt von

M.Sc. Ray Wollenzien

geboren am 17.01.1984 in Rathenow

Göttingen im März 2021 
1. Gutachter: $\quad$ Prof. Dr. Achim Dohrenbusch

Abteilung Waldbau und Waldökologie der gemäßigten Zonen

Fakultät für Forstwissenschaften und Waldökologie

Georg-August-Universität Göttingen

2. Gutachter: $\quad$ Prof. Dr. Norbert Lamersdorf

Abteilung Ökopedologie der gemäßigten Zonen

Fakultät für Forstwissenschaften und Waldökologie

Georg-August-Universität Göttingen

Tag der mündlichen Prüfung: $\quad$ Freitag, den 27.08.2021 


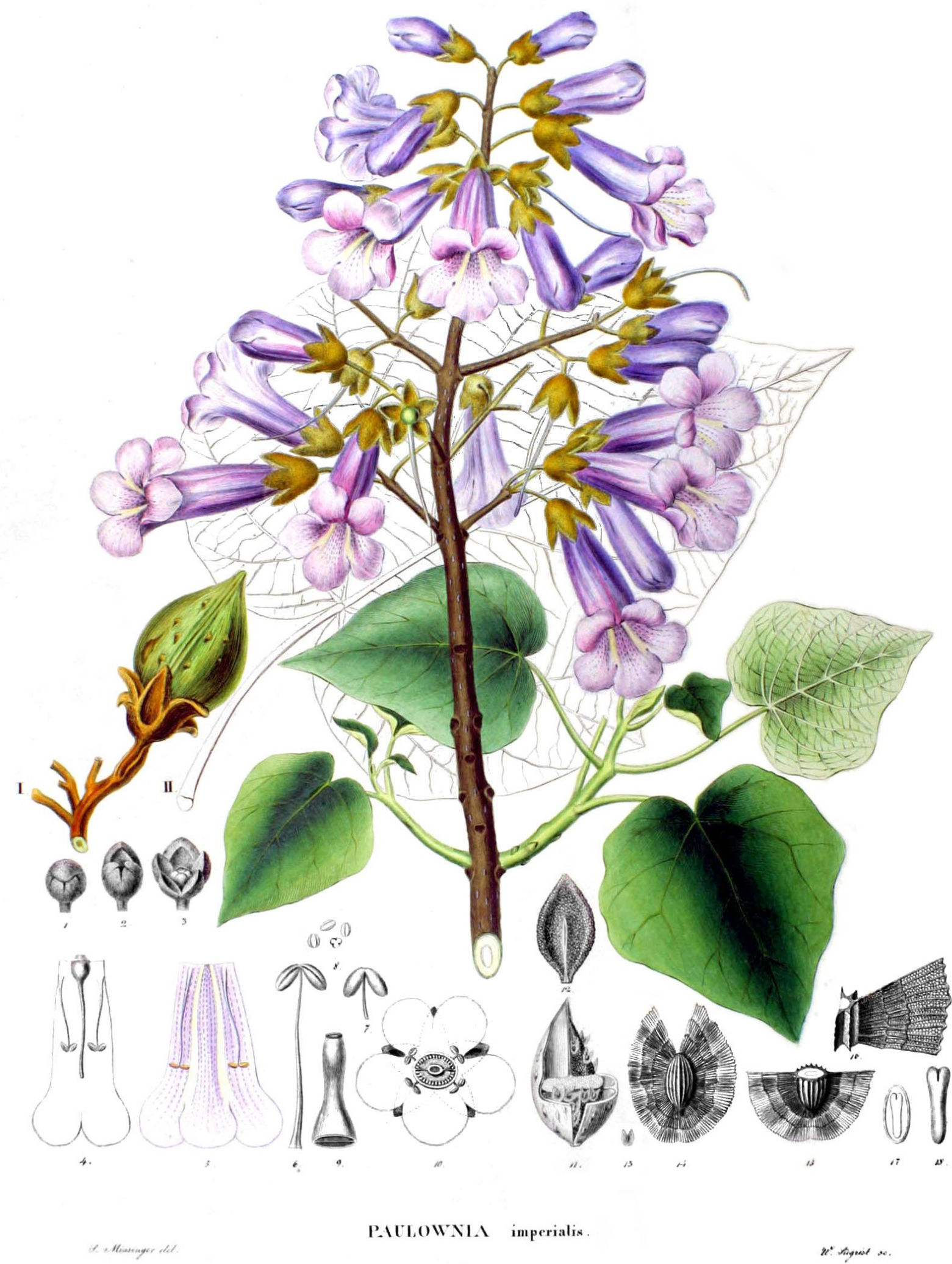

Abb. 1: Paulownia imperialis, Bildquelle: https://deacademic.com/pictures/dewiki/80/Paulowni_imperialis_SZ10.jpg 


\section{Vorwort}

Das Verfassen einer Monographie ist verständlicherweise ein langwieriger Prozess, der in meinem Falle die sieben Jahre schon überschritten hat. Für jeden leicht nachvollziehbar, weil vielleicht am häufigsten gebraucht, ist das Bild eines steilen, steinigen und vor allem unbekannten Weges.

Vermutlich ist es die Kernaufgabe einer Promotion bzw. dieses Lebensabschnittes diesen Weg zu beschreiten und das hier vorliegende Manuskript ist nur ein kleiner, aber nicht unbedeutender vorletzter Schritt der Reise?!

Ich glaube, dass wir an den Universitäten, meiner Erfahrung nach, über keine sehr ausgeprägte Fehlerkultur verfügen. Dabei wären doch genau die Momente, wo das erste Stolpern kam, das große Zaudern begann, die Unsicherheiten überhandnahmen und die Probleme anfänglich unüberwindbar erschienen und sich dann vielleicht doch nicht lösen, aber zumindest irgendwie umgehen ließen - genau über diese Punkte und die sehr individuellen Lösungen und Wege, die sich dabei auftaten - darüber wird zu oft geschwiegen, viel zu selten berichtet.

Dabei sollten wir den Mut haben genau darüber zu sprechen, auf das Andere daraus lernen und wir uns gegenseitig helfen können. Und das nicht nur um uns, sondern gerade auch die Wissenschaft für die Allgemeinheit zu verbessern, indem wir sie dadurch vor allem verständlicher und menschlicher gestalten.

So will ich also die mir hier gegebene einmalige Gelegenheit (ganz gewiss ist dies hier definitiv die erste und letzte Dissertation, die ich jemals zu schreiben gedenke) nutzen und offen eingestehen, dass sich von der Ursprungsidee zu der gesamten Promotion vielleicht nur gefühlte $10 \%$ des Kernthemas bis zum Schluss erhalten haben. Angedacht war mal eine wissenschaftlich begleitete Kooperation mit einem Privatunternehmen und Pflanzenteile sollten gefrostet und deren Überlebensrate bestimmt werden. Leider konnten die dafür benötigten Kühlkammer-Kapazitäten nie bereitgestellt werden. So lag also alle Hoffnung auf den Freilandversuchen, die dann aber binnen weniger Jahre die volle Härte der Natur zu spüren bekamen und von unerwartet hohen Ausfallzahlen gezeichnet waren, was die Datenausbeute sehr schmälerte. Ein ergänzender Gewächshausversuch zeigte auch nicht die erhofften Pflanzenreaktionen und so musste ich mich mit nur Wenigem zufriedengeben und hatte doch so viel erhofft, gar erwartet.

Die frühere Präsidentin der Universität, Frau Prof. Dr. Ulrike Beisiegel, fragte mich mal in einem Gespräch ganz beiläufig, ob ich denn jemals damit zufrieden sein könnte, meine Promotion nur so halb und nebenbei zu gestalten, weil die andere Hälfte, eigentlich ja der größere Teil des Tages, der Arbeit als Dozent gewidmet werden musste. Und ob ich es dann nicht lieber ganz bleiben lassen und mich der Arbeit im Wissenschaftsmanagement verschreiben sollte?

Eine klare Antwort blieb ich ihr damals schuldig, und ihre Worte wirkten lange in mir nach. Nun, fast am Ende des Weges angekommen, nach vielen schlaflosen Nächten und sehr vielen Selbstzweifeln, fällt mir eine Antwort doch relativ leicht. Im Grunde bin ich doch sehr zufrieden, über das was mir, in der Zeit, die vom Tage übrigblieb, möglich war und über das, was ich bis hierher erreichen konnte - und das empfinde ich als sehr viel. 
Den langen Weg von Anfang bis Ende begangen zu haben, zu stolpern, unzählige Male auf die Nase zu fallen und dann doch immer wieder weiter zu tippeln, zeigt ja entweder schon ein Stück Lebenskunst oder zumindest verdammt viel Sturheit. So oder so hat es mich bis hierher gebracht, und allein hätte ich es bestimmt nicht schaffen können Denn bei alldem habe ich unglaublich viel gelernt und das vor allem mit und durch die vielen tollen Leute, dir mir auf dem Weg begegnet sind und die mich mit ihren Ideen und ihrem ganzen Wesen immer wieder bereichert haben. Beispielsweise durfte ich fast zwei Dutzend Abschlussarbeiten in der Zeit mitbetreuen, und ein großer Teil davon gestaltete das Fundament, aus dem sich das vorliegende Manuskript erbaut, mit.

Bevor ich aber diesen Menschen ansatzweise versuchen will zu danken und dabei hoffentlich nicht allzu viele vergesse - noch ein paar abschließende Worte zur eigentlichen Thematik:

Das Anbaupotential der Gattung Paulownia unter mitteleuropäischen Klimabedingungen erschließt sich der Forschung erst sehr langsam, was ganz allgemein dem, meiner Meinung nach, noch viel zu wenigen Experimenten im Zusammenhang mit neuartigen Baumarten geschuldet ist. Die nächsten Jahre und Jahrzehnte werden von der Wissenschaftsgemeinde neue, bessere und vor allem schnellere Lösungen zum Erhalt der Wälder, unter den sich abzeichnenden Klimaänderungen, verlangen. Ein neues Denken wird hoffentlich nicht zu spät Einzug finden, und vielleicht leistet dieses Manuskript gar einen kleinen Beitrag dazu?!

\section{Danksagung}

Zuallererst möchte ich Prof. Dr. Achim Dohrenbusch für seine Idee zu dieser Thematik danken, denn sie war der Ausgangspunkt meiner Reise. Gemeinsam hatten wir in all diesen Jahren so einige, zumeist sehr intensive Diskussionen, in denen er mir immer wieder wertvolle Anregungen mitgab, die ich dann auch soweit irgend möglich umsetzte. Das wäre nicht so einfach möglich gewesen, wenn er nicht vom ersten Tage an, ein so offenes und freundliches Gemüt gezeigt hätte, welches es mir ermöglichte mit inm im alltäglichen (ich als Dozent - er als Chef) und im fachlichen (ich als „FreizeitPromovierender" - er als Chef) einen steten Austausch fast auf Augenhöhe führen zu können. Ganz gewiss habe ich es inm dabei nicht immer leicht gemacht - er mir aber auch nicht. Und er weiß ja gar nicht wie oft er mir in all den Jahren, in so vielen Situationen ein Vorbild war und es auch immer sein wird. In diesem Sinne bin ich inm für so vieles dankbar.

Begonnen hat meine fachliche Reise mit der handfesten Unterstützung meiner ersten BSc-Studentin Laura Nobis. Gemeinsam haben wir uns nach Brandenburg auf die erste Paulownia-Plantage gewagt und der Thematik zum ersten Mal angenähert. Trotz kleinerer Rückschläge haben wir uns dabei nicht entmutigen lassen und sind den Weg gemeinsam ein Stück gegangen. 
Zusammen mit Raphael Göller habe ich die Versuchsanlage auf der wunderschönen Insel Föhr, auf dem Land seiner Familie, errichtet. Sein steter Wille zur Arbeit, seine enorme Gastfreundschaft, seine ganze menschliche Natur haben mich durchweg fasziniert. Wie kalt ist doch die Nordseeküste und wie gut tut doch ein wärmender Ofen und ein steter, nie endender Nachschub an Kalthopfenschorle in Verbindung mit wunderbar tiefsinnigen Gesprächen und sanftmelodischem Friesenrock-Radio.

Sowohl die Versuchsanlage in Göttingen als auch in Brandenburg habe ich zusammen mit Florian Büker errichtet. Er ist mir in all den Jahren ein wirklicher Freund geworden, den ich nicht mehr missen will. Umso mehr hat es mich dann auch gefreut, dass er sich in seiner BSc-Arbeit mit meinen Pflanzen beschäftigt und dabei einen wichtigen Grundstein für dieses Manuskript hier gelegt hat.

Für die viele beispiellose Arbeit beim Aufbau der Gewächshausversuche gilt mein Dank besonders Franziska Dannenberg und David Runge, die beide ihre Masterarbeit der Paulownia gewidmet haben.

Eines Tages kam Prof. Dohrenbusch mit ernster Miene in mein Büro und fragte, ob ich die BSc-Arbeit von Fritz Hofheinz wirklich durchgeschaut hätte, bevor sie eingereicht wurde? Also ehrlich gesagt, habe ich natürlich immer versucht die Betreuung von Abschlussarbeiten ernst zu nehmen und dafür auch zu jeder Tages- und Nachtzeit die nötigen Stunden aufzubringen, vor allem zum Ende hin, wenn es bei allen immer etwas knapp wird. Aber bei aller Bescheidenheit bin ich nur wenig schuld daran, dass die BScArbeit von Fritz eine der besten wurde, die Prof. Dohrenbusch, nach eigenem Bekunden, je gelesen hatte. So haben einige der genialen Gedankengänge von Fritz auch in dieses Manuskript indirekt Einzug gefunden. Und so bin ich inm natürlich auch für mehr als nur eine tolle Zusammenarbeit, die weit über den DT-, WG- und sonstige Keller hinausging, dankbar.

Am Ende der ersten Vegetationsperiode brauchte ich dringend Hilfe beim Abernten der Biomasse der Gewächshauspflanzen und was bin ich froh darüber, dass Anne Wortmeier mich so viele Stunden dabei unterstütze und dass, obwohl nur ein kleiner Teil davon die Datengrundlage für ihre BSc-Arbeit bilden sollte. Allerdings nehme ich es ihr noch immer ein wenig übel, dass sie eine kleine Unachtsamkaeit meinerseits ausnutzte, um das ach so große Rätsel um mein Geburtsdatum zu lüften. Ihr ewiges Stillschweigen konnte ich mir nur mit einer persönlichen Postkarte vom afrikanischen Kontinent aus teuer erkaufen ;-)

Im zweiten Jahr der Gewächshausversuche gab es so viel Arbeit, die ich, neben meiner Vollauslastung durch die $75 \%$ Dozentenstelle, niemals ohne die Hilfe von Corinna Hoffmann und Daniel Eckardt hätte leisten können. Wir waren echt ein tolles Trio und die beiden ein Spitzen-Duo und vermutlich war der Spaß, den unserere gemeinsame Arbeit uns bereitete, noch weit über das Gewächshausareal hinaus zu hören. Corinna hatte im Übrigen den Mut, sowohl ihre BSc- als auch ihre MSc-Arbeit der Paulownia zu widmen und viele ihrer entworfenen Grafiken haben mich so manche statistische Nachtstunde beschäftigt. Einen großen Dank euch beiden für so viel Lebensfreude im selbstgezüchteten Dschungel!

Die Betreuung und Bewertung der Masterarbeit von Kai Thielen erlaubte mir einen Ausflug und Einblick in die Nachbarfakultät der Agrar. So konnte ich also mal einer mündlichen Verteidigung von der anderen Seite des Schreibtisches aus beiwohnen, was auch seinen Reiz hat. Kai hat mir bei vielen Gedankengängen zur Praxis maßgeblich weitergeholfen, wofür ich inm sehr dankbar bin. 
Abschließend muss ich beim Thema Betreuung noch die BSc-Abeit von Lang Lang (alias Jan Wieting) erwähnen. Zwar hat sie thematisch nicht das Geringste mit meinem Manuskript zu tun, aber Lang Lang hat mich gelehrt, dass aus Lebensfreude, Intelligenz und ein wenig Druck, wenn die Zeit knapp wird, so manches Mal kleine Diamanten entstehen können. Danke dafür alter Knabe.

Natürlich wäre all meine Arbeit, sowohl im Felde als auch im Gewächshaus nicht möglich gewesen ohne die vielseitige fachliche wie auch technische Unterstützung seitens meiner liebgewonnenen Waldbau-Abteilung. Stellvertretend gilt mein Dank hierbei vor allem Michael Unger, der jederzeit und egal bei welcher meiner Fragen immer die gleiche Antwort für mich parat hielt: „naayyyhhhhhh“.

Mit Manuel Punzet verbindet mich weit mehr als nur die Tatsache, dass er zeitgleich mit mir ein Doktorand von Prof. Dohrenbusch war. Neben den sehr vielen Stunden des fachlichen Austausches, haben wir uns auch immer wieder gegenseitig handfeste Unterstützung gegeben. Sei es nun, dass ich sein Sicherungsseil gehalten habe, oder er meine technische Ausrüstung gemanagt und gewartet hat. Langweilig wurde uns dabei nie, und so konnten wir in diesen lehrsamen Stunden viel voneinander profitieren. Unerwartet, aber mit viel Neugierde durfte ich dann alsbald das Büro mit Jessica RebolaLichtenberg und Maximilian Rößner teilen. Euch beide habe ich wirklich ins Herz geschlossen, und das lag nicht nur an den vielen ausufernden MontagvormittagGesprächen, bei denen uns Gott und die Welt thematisch nie genug waren. Immer hatten wir auch ein paar tröstende Worte füreinander parat, wenn der Uni-Alltag jeden von uns regelmäßig in den Wahnsinn trieb. Vor allem bei den viel zu seltenen „Mariaspring“Gesprächen mit Max, zirkelten wir die großen Fragen des Lebens ab, und die Erinnerung daran wird mir hoffentlich auf immer ein Quell langwährender körperlicher und geistiger Gesundheit bleiben.

Alsbald hat dann auch Liane Neudam ihren Weg in unser Waldbau-Team gefunden. Sie war mir gerade in den letzten Monaten und Wochen immer wieder eine liebgewonnene Gesprächspartnerin, der gegenüber ich auch mal das eine oder andere Wort zum Thema - steiniger Weg und so - loswerden konnte. Leider konnte ich ihre guten Ratschläge nicht immer sogleich umsetzen, aber ich war doch immer stets bemüht auch mal ein paar sonnige, leider viele zu kurze Momente außerhalb meines selbstgewählten Kellerverlieses, auf ihr anraten hin, zu erhaschen.

Sehr großen Dank muss ich unbedingt Dominik Seidel aussprechen, dem ich egal wann immer mit meinen Fragen, Bemerkungen und sei es manchmal auch nur der Wunsch nach einem kurzen Gespräch, belästigen konnte. Sein Fachwissen, sein Humor und vor allem seine warmherzige, menschliche Art haben mich immer wieder aufgerichtet und mir geholfen den Weg weiterzugehen. 
Neben dem beruflichen Alltag als Dozent und dem Freizeitvergnügen als Promovierender, war es doch immer wieder eine Überraschung für mich, wenn es schon wieder Mittwochabend und Zeit für eine weitere Übungsstunde beim Jagdhornbläserchor der Forstlichen Fakultät wurde. Ganz plötzlich war mein Kopf wieder frei und konnte, so eben noch versunken in den tiefen Nebeln irgendwelcher statistischer Analysen, wieder emporschnellen und Notenblätter lesen und sich der melodischen Abfolge französischer Jagdmusik hingeben. Für diese Flucht in eine andere so wohlklingende Welt (die ich jedem jederzeit empfehlen würde) muss ich, stellvertretend für die ganze Truppe, vor allem dem Leiter des Chors Andreas Buck-Gramcko und meinem Freund Robert Grenzdörffer danken. Gerade Robert hat sich ja in vielen privaten Stunden der Aufbauarbeit meines so rudimentär ausgeprägten Musikverstandes gewidmet. Und ob das wirklich was gebracht hat, ist auch weiterhin fraglich? Naja, zumindest gab es nie Beschwerden bei den aberdutzenden von Disputations-Feiern, zu denen wir so regelmäßig gemeinsam geblasen haben.

Der Tatsache, dass im vorliegenden Manuskript nicht allzu viele Rechtschreibfehler zu finden sind, habe ich einzig und allein Gaby Jürgens zu verdanken. Sie hat es wirklich immer wieder binnen Stunden geschafft, sich durch die vielen Seiten, wofür ich Tage, wenn nicht gar Wochen gebraucht hätte, zu wühlen und noch so jedes kleinste fehlende Komma zu finden. So muss ich ihr einen großen Dank für so viel Freude beim Finden und Rot-Markieren meiner Unzulänglichkeiten aussprechen.

Fast schon seit Beginn meiner Zeit in Göttingen, konnte ich immer wieder auf die Weisheiten meines Freundes Falk Müller-Braun bauen. Er hat in so vielen schwierigen Situationen fern des Uni-Alltags zu mir gehalten, was inm natürlich nicht immer leicht fiel und mir doch so viel bedeutet hat. Ich danke dir, dass du mich trotz meiner Dummheiten, nur selten, aber verdient über den Jordan getrieben und wieder zurückgelassen hast.

In all den vielen Jahren hatte ich immer eine große moralische Stütze, mein allzeit bereites schlechtes Gewissen ad persona vertreten durch meinen alten Schulfreund Jörg Heise. Jedes Jahr trafen wir uns wieder am gleichen Orte, zu unserem „Kinderurlaub“ (seine Tochter Lina = mein Patenkind), und er stellte mir jedes Jahr erneut die gleichen Fragen: „Und? Was hast du so erreicht? Wohin soll's gehen? Wie lange willst du noch brauchen?". Eine oft schmerzvolle Inventur, aber auch immer ein klarer, wohltuender Strich. Die Zeit verging, und der Weg wurde länger, aber Jörg hat im Grunde fortwährend an mich geglaubt - er war immer auf Seiten „Team-Ray“ und dafür kann ich inm nicht genug danken.

Zum Ende hin, möchte ich natürlich das Wichtigste nicht vergessen - die Familie. Allen voran meine so liebgewonnene Schwägerin Caterina Rönnert. So viele Stunden hat sie sich meine Daseinssorgen angehört und hatte immer einen guten Rat für mich parat, und ich war nicht verlegen um den ein oder anderen für sie.

Liebe Familie, was habt ihr mir in all den Jahren doch für Sorgen, Nöte, Kopfschmerzen und schlaflose Nächte bereitet?!

Was bin ich so unendlich dankbar dafür, dass ich euch habe.

Denn mal ganz ehrlich, was wäre ich ohne euch!

Und dann gibt es da noch zwei so wunderbare Wesen - meine Freundin Anica und unser gemeinsamer Hund Ida - die erst sehr spät, aber doch im genau richtigen Augenblick in meine Welt getreten sind. Was freue ich mich darauf zu sehen, was die Zukunft für uns drei bereithält. 
Wobei ich auch sehr gespannt bin, was für ein Mensch ich sein werde, wenn diese langjährige Reise, dieser lange, steinige Weg sein Ende gefunden hat und wie es von dort aus weitergehen wird. In diesem Sinne also auf, auf in die Neue Welt ;-)

Zum Abschluss meiner persönlichen Worte, will ich mir noch eine kleine Metapher erlauben:

Das Schreiben einer Dissertation habe ich mal als den Bau eines Segelbootes, beschrieben, das im Grunde schon vom ersten Tage an auf dem Fluss treibt. Nur nirgends gibt es einen Bauplan und irgendwann soll es auf das offene Meer hinausgehen, weil alle Flüsse früher oder später dort enden. Also heißt es sofort und Stück für Stück an die mühevolle, mehrjährige Arbeit zu gehen.

Doch wie wird dieses Boot jemals aussehen, geschweige denn seetüchtig?

Und selbst wenn eine ungefähre Vorstellung besteht, dann wird es mit Sicherheit trotzdem ganz anders werden, wie zu Anfang schon beschrieben.

Woher jetzt eigentlich dieser Vergleich mit dem Bootsbau stammt?

Nun ja, die letzten Monate und erst recht die letzten Wochen haben sich so angefühlt, als ob ein Loch nach dem anderen in der Bordwand zu stopfen war. Immerzu muss irgendwie das viele Wasser von drinnen wieder nach draußen geschöpft werden, was stetig über die noch zu kurze Bordwand herein schwappt.

Und ständig dieses Gefühl kurz vor dem Ertrinken zu stehen.

Jetzt bloß nicht die Laune verlieren,

einfach weiter machen - Schritt für Schritt - einen Eimer Wasser nach dem anderen, noch ist die Nase über der Wasseroberfläche - nur immer weiter ...

Und dann kommt auch schon viel zu schnell die Mündung des Flusses in Sicht.

Die ersten Wellenberge des offenen Meeres werden spürbar.

Ist es wirklich schon so weit?

Sind alle Löcher gestopft - werden sie halten??

Wird es schwimmen???

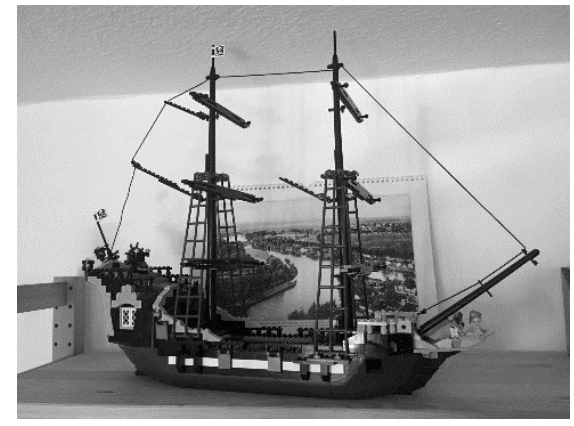

Gott sind Wellen und Wind,

aber Segel und Steuer sind euer!1

\footnotetext{
${ }^{1}$ Mir zur Lebensweisheit geworden und ursprünglich angelehnt an den Ausspruch von Gorch Fock: „Gottes sind Wogen und Wind, Segel aber und Steuer, dass ihr den Hafen gewinnt, sind euer." (Gorch Fock, eigentlich Johannes Kienau, 1880-1916, niederdeutscher Erzähler, Lyriker, Elbfischer und Marinesoldat, gefallen in der Seeschlacht beim Skagerrak.)
} 


\section{Zusammenfassung}

\section{Einleitung}

Die Paulownia ist eine der ältesten vom Menschen aktiv angebauten Baumgattungen der Welt. Erste schriftliche Erwähnungen gehen bis 1000 v.Chr. zurück (Barton, 2007). Eine genaue Bestimmung der natürlichen Verbreitung ist, aufgrund der jahrhundertelangen, anthropogenen Streuung der verschiedenen Arten heutzutage nicht mehr möglich. Aber auf Grundlage der ökologischen Amplitude der Gattung lässt sich das natürliche Verbreitungsgebiet von Indochina über Taiwan und China bis nach Korea und Japan beschreiben.

Aus der Gattung Paulownia haben sich, durch die geographische Isolation in sich stark voneinander unterscheidenden Klimaregionen und die damit verbundene natürliche Anpassung an die unterschiedlichen Umwelteinflüsse, verschiedene Arten herausgebildet. Die Taxonomie ist auch bisher nicht zweifelsfrei geklärt, wobei in der Mehrzahl der jüngeren Literatur die Bildung einer eigenen Familie der Paulowniaceae (Blauglockenbaumgewächse) und eine Einteilung in sieben Arten (eFloras, 2014) favorisiert wird.

Der Baum und mit inm sein leichtes und formstabiles Holz, die weiß-lila Blüten und die großen Blätter, sind tief in der asiatischen Mythologie verankert und es wird mit ihnen Stärke, Reinheit und Erneuerung verbunden (Lacy, 2000).

Um 1830 gelangte nachweislich das erste Exemplar der Gattung nach Europa. Heutzutage sind Paulownia-Baumarten in jedem Land des Kontinents zu finden, wobei die Bäume nur selten in den für sie natürlichen Vegetationstypen wie bspw. Auenstandorte und Waldlichtungen anzutreffen sind (Essel, 2007). In vielen europäischen Länder wird aber von sich natürlich verbreitenden Bäumen berichtet, die oft im Zusammenhang mit bereits vorhandenen Plantagenbäumen stehen (Essel, 2007). Im deutschsprachigen Raum wurde das erste verwilderte Vorkommen (P.tomentosa) bereits um 1925 beschrieben (Kiermeier, 1977). In den Bundesländern BadenWürttemberg, Hessen, Nordrhein-Westfalen gilt sie heute als eingebürgert (Buttler and Thieme, 2013). In Berlin, Bayern, Niedersachsen und Rheinland-Pfalz wurden noch weitere bisher unbeständige Vorkommen gesichtet, wobei es sich dabei zumeist um Grenzstandorte in urbanen Bereichen wie bspw. Bahndämme, Mauerritzen, usw. handelt (Keil and Loos, 2004).

Die häufigste Form der Verbreitung erfolgt durch den Anbau von Hybriden auf Kurzumtriebsplantagen (Cathaia, 2020). Auch werden die verschiedenen Arten seit vielen Jahren im Pflanzenhandel angeboten (Stimm et al., 2013) und finden auf diese Weise Einzug in die deutschen Vorgärten und von dort aus weiter in die freie Natur.

\section{Fragestellung}

Bei jeder Kulturbegründung ist die Phase des Anwuchses besonders kritisch für die Pflanzen. Dabei sind für den Anwuchserfolg sehr viele unterschiedliche Faktoren bedeutend, wie z.B. die Baumart, ihre Provenienz, der Zustand des Pflanzmaterials usw. Von besonderer Wichtigkeit sind aber die Witterungsbedingungen zum Zeitpunkt der Pflanzung, als auch in den Wochen und Monaten danach. Die in den letzten Jahren immer häufiger auftretenden saisonalen Trockenereignisse gefährden den Anwuchserfolg der Pflanzen zunehmend. 
Dieses Manuskript widmet sich mittels einem Freiland- und einem Gewächshausversuch der Beantwortung unterschiedlicher und sich ergänzender Fragestellungen zu dieser Problematik. Aufgrund ihrer Wuchseigenschaften und der Häufigkeit ihres weltweiten Anbaus wurden dafür die drei P.-Arten: P.elongata, P.fortunei und P.tomentosa als Untersuchungsobjekte ausgewählt.

In einem dreijährigen Freilandversuch werden die Wachstumsreaktionen der drei P.Arten unter mitteleuropäischen Klimabedingungen im Vergleich zueinander beobachtet. Der zweijährige Gewächshausversuch ergänzt und erweitert diese Fragestellung, indem das Wachstumsverhalten unter Beeinflussung verschiedener Belichtungs- und Bewässerungsstufen betrachtet wird.

\section{Methodik}

Zum experimentellen Anbau im Freiland wurden drei von den Boden- und den Klimaverhältnissen her unterschiedliche Standorte ausgewählt, die sich im westlichen Brandenburg, in Süd-Niedersachsen und auf der Nordseeinsel Föhr befinden.

Von den Bodenverhältnissen ergab sich dadurch eine Variation von einer Braunerde über eine Rendzina aus Kalkgestein, bis hin zu einem Marschboden mit einer PodsolParabraunerde. Des Weiteren konnten sowohl ein Kontinental- als auch ein Übergangsklima abgebildet und um ein raues, klar atlantisch beeinflusstes Seeklima ergänzt werden.

Auf den Versuchsflächen wurden Plots der drei P.-Arten zu gleichen Anteilen gepflanzt. Die Plots wurden vorab in vier verschiedene Behandlungsgruppen unterteilt, um einen möglichen Wachstumseffekt durch die Verwendung von dem Bodenhilfsstoff Geohumus bzw. einer Mykorrhizierung beobachten zu können.

Aufbauend auf den ersten Erfahrungen im Freiland wurde die Fragestellung mit Hilfe eines Gewächshausversuches ergänzt. Dazu wurden Keimlinge der drei P.-Arten vereinzelt in Töpfe gepflanzt und ihr Wachstum in einem überdachten, vollverglasten und nach außen hin offenem Gewächshausabteil beobachtet. Die Pflanzen wurden vorab in drei verschiedene Belichtungs- und Bewässerungsstufen mit der jeweiligen Abstufung von $100 \mathrm{zu} 60 \mathrm{zu} 40 \%$ unterteilt. Insgesamt ergaben sich folglich 27 verschiedene Behandlungsstufen (3 Pflanzenarten $\times 3$ Lichtstufen $\times 3$ Wasserstufen). Die Lichtabstufungen wurden mittels Beschattungsnetzen realisiert und die Bewässerung orientierte sich mit ihrem $100 \%$ Wert an dem Jahresniederschlag in Rheinland-Pfalz.

\section{Ergebnisse}

Der Anbau von Paulownia ist unter mitteleuropäischen Klimabedingungen möglich, wie die Freilandversuche gezeigt haben. Auf die immer häufiger auftretenden Frühjahrs- und Sommertrockenheit reagieren die jungen Paulownia-Pflanzen jedoch mit hohen Ausfallraten. Der Anwuchserfolg ist folglich stark von den vorherrschenden Witterungsverhältnissen abhängig.

Beim Längenwachstum blieben die drei P.-Arten weit hinter den Erwartungen, die im Allgemeinen mit dieser Gattung verknüpft werden, zurück. Die verschiedenen Behandlungsarten, unter Einsatz von Geohumus und Mykorrhizierung, haben keinen nachweislichen Effekt auf das Wachstum der Pflanzen genommen. 
Im Gewächshaus haben die P.-Pflanzen verschiedenartig auf die gegebenen Licht- und Wasserverhältnisse reagiert. Sowohl das Längen- als auch das Durchmesserwachstum nehmen bei einer Verringerung des Lichtes zu. Dabei verschiebt sich aber das Verhältnis von Höhe zu Durchmesser und es entwickeln sich bei starker Beschattung lange, aber dafür dünne Pflanzen.

Die Wasserverfügbarkeit hat keinen nachweislichen Effekt auf das Längenwachstum. Jedoch zeichnet sich ein Zusammenhang zwischen der gegebenen Wassermenge und der Entwicklung des Pflanzendurchmessers ab.

Ein Rückgang der Wasserverfügbarkeit ist mit einer Verringerung der Stängelbiomasse und kleineren Blattflächen verbunden. Auch im Gewächshaus haben sich keine Unterschiede zwischen den drei P.-Arten gezeigt.

\section{Schlussfolgerungen}

Um den Anwuchserfolg zu sichern und die Ausfallraten im Freiland gering zu halten, empfiehlt sich eine Bewässerung der Kultur oder die Pflanzung älterer aber stabilerer Pflanzenindividuen (Cathaia, 2020; EnPf, 2021; WeGrow, 2020), was wiederum die Anbaukosten erhöhen würde.

Um arttypische Unterschiede und einen möglichen positiven Einfluss von Bodenhilfsstoffen (Mehrotra, 1996; Mehrotra et al., 1998; Mehrotra, 1997a) im Wachstum der P.-Pflanzen nachweisen zu können, bedarf es voraussichtlich eines längeren Beobachtungszeitraumes der über drei Vegetationsperioden hinaus geht.

Die Keimung, als auch das spätere Heranreifen der Samen zu Jungpflanzen, erfordert intensiven Lichtgenuss, da laut Zhu et al. (1986) bereits eine Beschattung um $70 \%$ tödlich auf die Verjüngung wirken kann. Die maximale Beschattung im Gewächshaus erreichte $60 \%$ und schon bei dieser Stufe zeigten sich hohe Ausfallzahlen im Verlauf der zweiten Vegetationsperiode.

Aufgrund dieser geringen Schattentoleranz erscheint es mehr als fraglich ob Paulownia das Potential zu einer natürlichen Verbreitung in mitteleuropäischen Wälder besitzt (Bork et al., 2015). Mosandl and Stimm (2015) gehen davon aus, dass sie im Verlauf der natürlichen Sukzessionsprozesse der Wälder verdrängt werden würde.

Einmal abgesehen von den Ziergehölzen in den Vorgärten und der ungewollten Verbreitung in den Wäldern, sind die in Deutschland zurzeit denkbaren Landnutzungsysteme zum aktiven Anbau von Paulownia Kurzumtriebsplantagen und Agroforstsysteme.

Seit vielen Jahren findet bereits der Anbau von Paulownia-Baumarten und ihrer Hybriden auf Kurzumtriebsplantagen (KUP) in Deutschland statt (Cathaia, 2020, siehe auch WeGrow, 2020). Dabei besitzt die Gattung eine ganze Reihe von vorteilhaften Eigenschaften die sie im Besonderen als Plantagenbaum im Kurzumtrieb empfehlen, wie Zhu et al. (1991, 1986) ausführlich darlegen (siehe auch Hofheinz, 2016).

Bei der Anlage einer Paulownia-KUP braucht es keine Extraplanung da sie sich in Punkto Pflanzverfahren und Pflege nicht von anderen üblichen KUP-Gehölzen unterscheidet (Schildbach et al., 2009). Eine Paulownia-KUP besitzt durchaus ein hohes Potential das Landschaftsbild zu bereichern und in ökologischer Hinsicht aufzuwerten. Allerdings wäre hierfür eine Anpassung des rechtlichen Rahmens ratsam, um Paulownia in die Liste der förderfähigen KUP-Baumarten aufnehmen zu können (VwG Köln, 2014). 
Ein Anbau von Paulownia in Form von Agroforstsystemen (AFS) wird in China seit Jahrhunderten praktiziert (Wang and Shogren, 1992; Zhu et al., 1991, 1986). Erst seit den 2010er Jahren hat sich auch die Forschung zur Paulownia in Deutschland intensiviert (Bork et al., 2015; Felbermeier et al., 2015; Mosandl and Stimm, 2015; Stimm et al., 2013). Infolgedessen ist die Verwendung in AFS noch nicht sehr weit bekannt, verbreitet und erforscht.

Die Neu-Etablierung von AFS in Deutschland ist rechtlich noch nicht abschließend geklärt (Böhm et al., 2017; Chalmin and Möndel, 2009; Zehlius-Eckert, 2018). Der Anbau von Paulownia kann aber, unter Beachtung der in den Freilandversuchen ausführlich beschriebenen Anwuchsprobleme, ökologisch und ökonomisch sinnvoll sein (Möndel et al., 2009).

Seit geraumer Zeit sind P.-Arten mit einer gewissen Selbstverständlichkeit in der mitteleuropäischen Naturlandschaft zu finden. Die Anzeichen von Invasivität, wie sie bspw. nach Waldbränden in den USA, in Form von Massenvermehrungen, beschrieben werden (Innes, 2009; Remaley, 2005), sind laut Nehring et al. (2013) aber nicht einfach auf mitteleuropäische Verhältnisse übertragbar. Die Autoren Vor et al. (2015) beschreiben fünf Kriterien um eine mögliche Gefahr der Invasivität bewerten zu können, die in Teilen durchaus auch auf die Paulownia zutreffend sind. Daher verwundert es folglich nicht, dass bspw. die Baumart P.tomentosa als nicht anbauwürdig für die deutschen Wälder eingestuft wird (Vor et al., 2015). Einzelne Exemplare die sich auf natürliche Weise verbreitet haben, würden voraussichtlich schon allein durch die natürliche Sukzession der mitteleuropäischen Wälder verdrängt werden (Mosandl and Stimm, 2015). Ein Anbau auf landwirtschaftlichen Flächen ist, anders als es im Wald der Fall ist, weiterhin jederzeit möglich (EnPf, 2021; WeGrow, 2020). Ob dies im Laufe der Zeit eine Neubewertung hinsichtlich der potenziellen Invasivität (Nehring et al., 2013) nach sich zieht und Paulownia evtl. in ähnlicher Form wie die Art Robinia pseudoacacia bewertet wird (Vor et al., 2015), und sie somit als „bedingt anbauwürdig“ eingestuft wird, bleibt aber weiterhin fraglich.

\section{Summary}

\section{Introduction}

Paulownia is one of the oldest tree genera in the world actively cultivated by humans. First written records date back to 1000 BC (Barton, 2007). An exact determination of the natural distribution is no longer possible today, due to the anthropogenic dispersion of the different species over centuries. However, based on the ecological amplitude of the genus, the natural distribution area can be described from Indochina via Taiwan and China to Korea and Japan.

Different species have evolved from the genus Paulownia due to the geographic isolation in climatic regions that differ greatly from each other and the natural adaptation to the different environmental influences associated with this. The taxonomy has also not yet been clarified beyond doubt, with the majority of recent literature favouring the formation of a separate family of the Paulowniaceae (in German: Blauglockenbaum) and a division into seven species (eFloras, 2014).

The tree, and with it its light and dimensionally stable wood, white-purple flowers and large leaves, are deeply rooted in Asian mythology and are associated with strength, purity and renewal (Lacy, 2000). 
Around 1830, the first specimen of the genus is documented to have reached Europe. Today, Paulownia tree species can be found in every country on the continent, although the trees are rarely found in their natural vegetation types, such as floodplain sites and forest clearings (Essel, 2007). In many European countries, however, naturally spreading trees are reported, often in association with existing plantation trees (Essel, 2007). In German-speaking countries, the first feral occurrence (P.tomentosa) was already described around 1925 (Kiermeier, 1977). In the federal states of BadenWürttemberg, Hesse, North Rhine-Westphalia, it is now considered naturalised (Buttler and Thieme, 2013). In Berlin, Bavaria, Lower Saxony and Rhineland-Palatinate, other hitherto unstable occurrences have been sighted, most of which are border sites in urban areas such as railway embankments, wall cracks, etc. (Keil and Loos, 2004).

The most common form of spread is through the cultivation of hybrids on short-rotation plantations (Cathaia, 2020). The various species have also been offered in the plant trade for many years (Stimm et al., 2013) and are thus finding their way into German front gardens and from there further into the wild.

\section{Issue}

In every cultivation, the phase of establishment is particularly critical for the plants. Many different factors are important for successful establishment, such as the tree species, its provenance, the condition of the planting material, etc. However, the weather conditions at the time of planting as well as in the weeks and months afterwards are of particular importance. Of particular importance, however, are the weather conditions at the time of planting as well as in the weeks and months afterwards. Seasonal drought events, which have become more frequent in recent years, are increasingly endangering the success of plant growth.

This manuscript is dedicated to answering different and complementary questions on this problem by means of an field and a greenhouse experimental setup. Due to their growth characteristics and the frequency of their worldwide cultivation, the three Paulownia species: P. elongata, P. fortunei and P. tomentosa were selected as objects of study.

In a three-year field experimental setup, the growth reactions of the three Paulownia species are observed in comparison to each other under Central European climatic conditions. The two-year greenhouse experimental setup complements and expands this question by observing the growth behaviour under the influence of different light and irrigation levels.

\section{Methods}

Three study sites with different soil and climatic conditions were selected for experimental cultivation in the field. They are located in western Brandenburg, in southern Lower Saxony and on the North Sea island of Föhr.

The soil conditions varied from a brown earth to a rendzina of limestone to a marshy soil with a podsol-parabrown earth. Furthermore, both a continental and a transitional climate could be mapped and supplemented by a rough, clearly Atlantic-influenced maritime climate.

Study plots of the three Paulownia species were planted in equal proportions. The study plots were divided into four different treatment groups in advance in order to be able to observe a possible growth effect through the use of the soil additive Geohumus or mycorrhisation. 
Based on the first experiences in the field, the question was supplemented with the help of a greenhouse experimental setup. For this purpose, seedlings of the three Paulownia species were planted individually in pots and their growth was observed in a covered, fully glazed greenhouse compartment that was open to the outside. The plants were divided in advance into three different light exposure and watering levels with the respective gradation of 100 to 60 to $40 \%$. This resulted in a total of 27 different treatment levels ( 3 plant species $\times 3$ light levels $\times 3$ water levels). The light levels were realised by means of shading nets and the irrigation was oriented with its $100 \%$ value to the annual precipitation in Rhineland-Palatinate.

\section{Results}

The cultivation of Paulownia is possible under Central European climatic conditions, as the field trials have shown. However, the young Paulownia plants react with high failure rates to the increasingly frequent spring and summer droughts. Consequently, the growth success is strongly dependent on the prevailing weather conditions.

In terms of length growth, the three Paulownia species fell far short of the expectations generally associated with this genus. The various treatments, using Geohumus and mycorrhization, did not have any demonstrable effect on the growth of the plants.

In the greenhouse, the Paulownia plants reacted differently to the given light and water conditions. Both length and diameter growth increase when light is reduced. However, the ratio of height to diameter shifts and long but thin plants develop in the case of strong shading.

Water availability has no demonstrable effect on length growth. However, there is a correlation between the given amount of water and the development of the plant diameter.

A decrease in water availability is associated with a reduction in stem biomass and smaller leaf areas. No differences between the three Paulownia species were shown in the greenhouse either.

\section{Conclusions}

To ensure establishment success and keep failure rates low in the field, it is recommended to irrigate the crop or plant older but more stable plant individuals (Cathaia, 2020; EnPf, 2021; WeGrow, 2020), which in turn would increase cultivation costs.

In order to be able to prove species-specific differences and a possible positive influence of soil additives (Mehrotra, 1996; Mehrotra et al., 1998; Mehrotra, 1997b) in the growth of Paulownia plants, a longer observation period of more than three vegetation periods is probably required.

Germination, as well as the later maturation of the seeds into young plants, requires intensive light enjoyment, since according to Zhu et al. (1986), shading of as little as 70 $\%$ can have a lethal effect on regeneration. The maximum shading in the greenhouse reached $60 \%$ and already at this level high numbers of failures appeared in the course of the second vegetation period.

Due to this low shade tolerance, it seems more than questionable whether Paulownia has the potential to spread naturally in Central European forests (Bork et al., 2015). Mosandl and Stimm (2015) assume that it would be displaced in the course of the natural succession processes of forests. 
Apart from the ornamental shrubs in front gardens and the unintentional spread in forests, the land use systems currently conceivable in Germany for the active cultivation of paulownia are short-rotation plantations and agroforestry systems.

For many years, Paulownia tree species and their hybrids have been cultivated on shortrotation plantations (SRC) in Germany (Cathaia, 2020, see also WeGrow, 2020). The genus possesses a whole range of advantageous characteristics that recommend it in particular as a plantation tree in short rotation, as Zhu et al. $(1991,1986)$ explain in detail (see also Hofheinz, 2016).

The establishment of a Paulownia SRC plantation does not require any extra planning as it does not differ from other common SRC trees in terms of planting methods and maintenance (Schildbach et al., 2009). A Paulownia SRC plantation has a high potential to enrich the landscape and to enhance its ecological value. However, it would be advisable to adapt the legal framework to include Paulownia in the list of eligible SRC tree species (VwG Köln, 2014).

Cultivation of Paulownia in the form of agroforestry systems (AFS) has been practised in China for centuries (Wang and Shogren, 1992; Zhu et al., 1991, 1986). Only since the 2010s has research on Paulownia also intensified in Germany (Bork et al., 2015; Felbermeier et al., 2015; Mosandl and Stimm, 2015; Stimm et al., 2013). As a result, its use in AFS is not yet very widely known, disseminated and researched.

The new establishment of AFS in Germany has not yet been finally clarified legally (Böhm et al., 2017; Chalmin and Möndel, 2009; Zehlius-Eckert, 2018). However, the cultivation of Paulownia can be ecologically and economically sensible, taking into account the growth problems described in detail in the field experimental setup (Möndel et al., 2009).

For some time now, Paulownia species have been found in the Central European natural landscape with a certain degree of self-evidence. However, according to Nehring et al. (2013), the signs of invasiveness described, for example, after forest fires in the US, in the form of mass propagation (Innes, 2009; Remaley, 2005), cannot simply be transferred to Central European conditions. The authors Vor et al. (2015) describe five criteria for assessing a possible risk of invasiveness, some of which are also applicable to Paulownia. It is therefore not surprising that the tree species P.tomentosa, for example, is classified as not worthy of cultivation in German forests (Vor et al., 2015). Individual specimens that have spread naturally would probably be displaced by the natural succession of Central European forests alone (Mosandl and Stimm, 2015). Cultivation on agricultural land, unlike in forests, is still possible at any time (EnPf, 2021; WeGrow, 2020). Whether this will lead to a reassessment of the potential invasiveness (Nehring et al., 2013) over time and whether Paulownia will be assessed in a similar way to the species Robinia pseudoacacia (Vor et al., 2015) and thus be classified as "conditionally worth cultivating" remains questionable. 


\section{Inhaltsverzeichnis:}

VORWORT.

.II

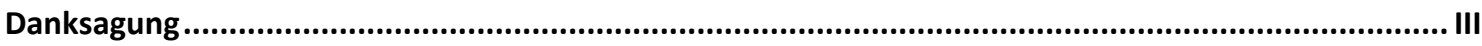

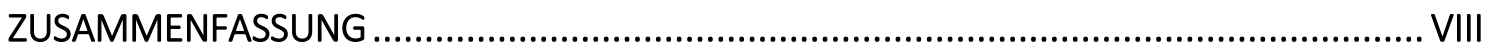

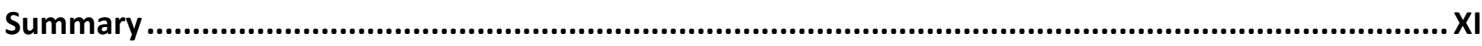

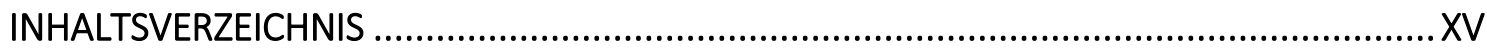

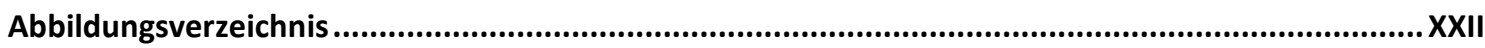

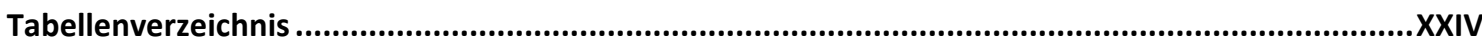

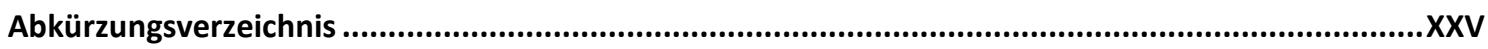

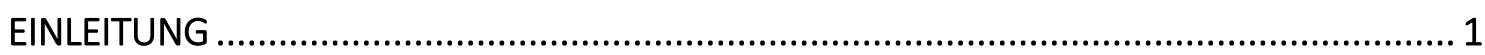

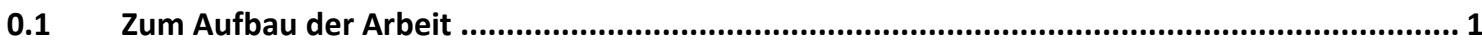

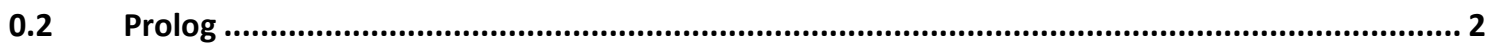

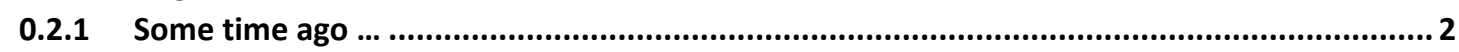

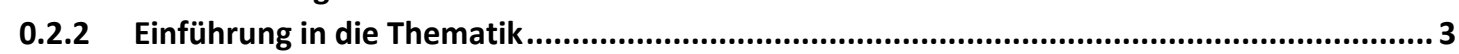

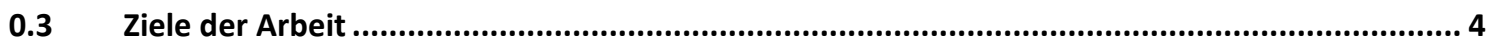

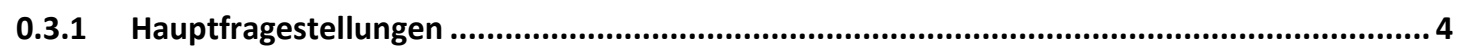

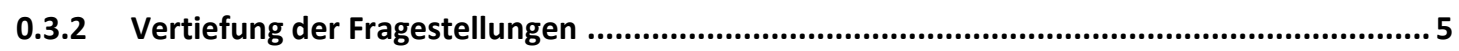

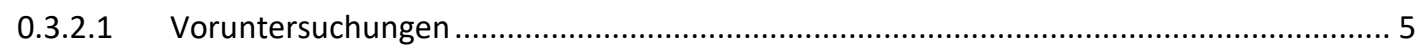

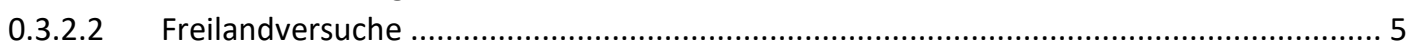

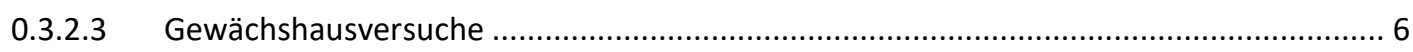

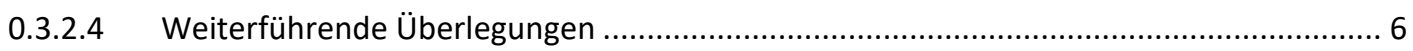

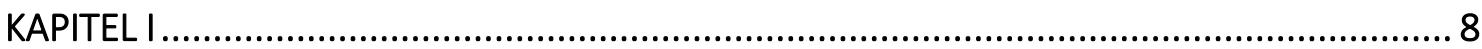

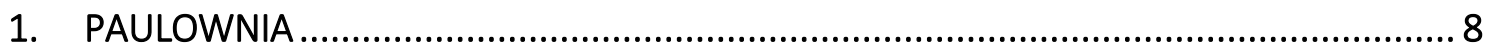

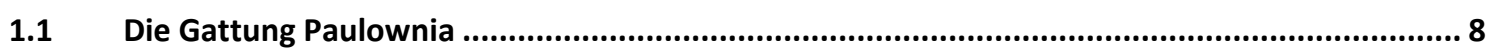

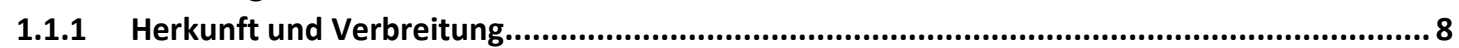

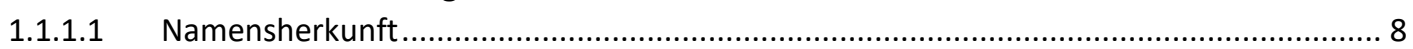

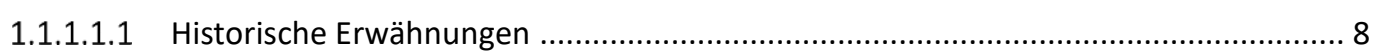

1.1.1.1.2 Entstehung des westlichen Namens .............................................................. 9

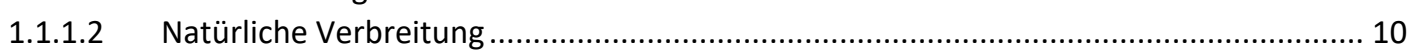

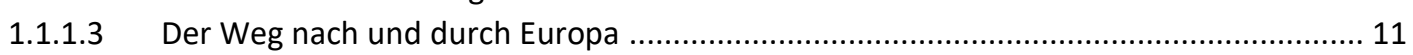

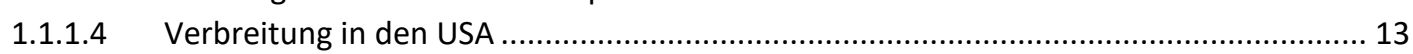

1.1.1.4.1 Wiedereinführung auf dem amerikanischen Kontinent........................................... 13

1.1.1.4.2 Anbau- und Invasivitätsproblematik in den USA ................................................ 14

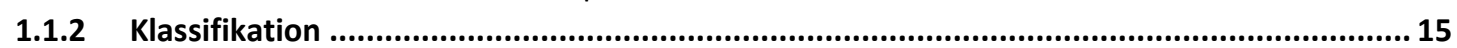

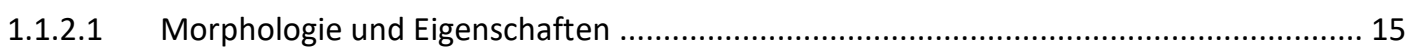

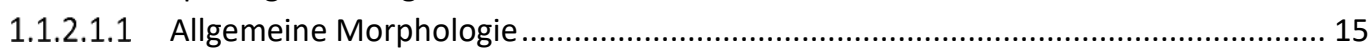


1.1.2.1.2 Taxonomische Eigenschaften zur Charakterisierung .......................................... 15

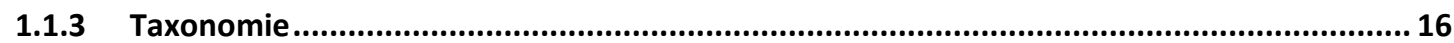

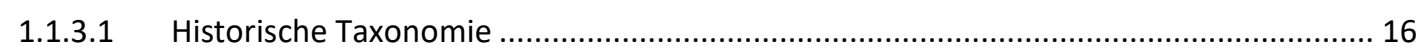

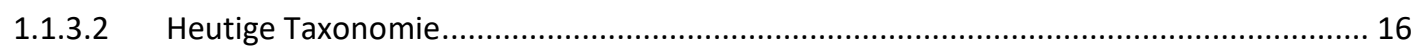

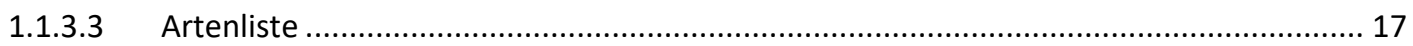

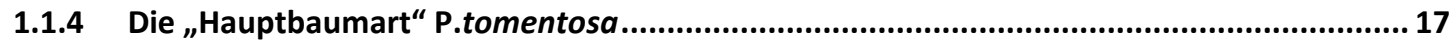

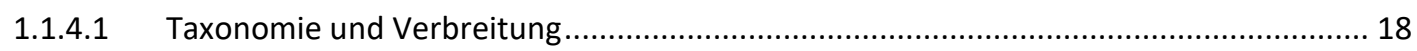

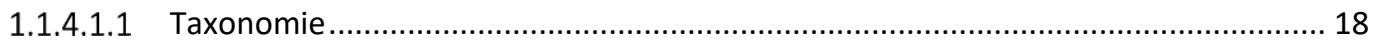

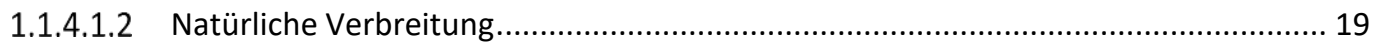

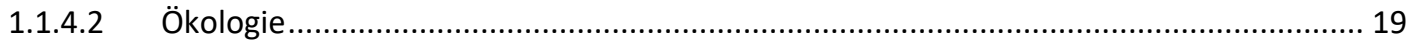

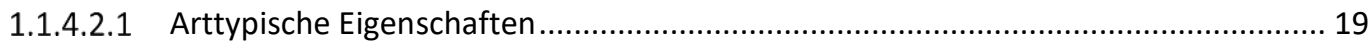

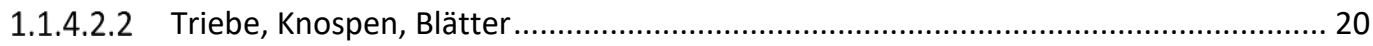

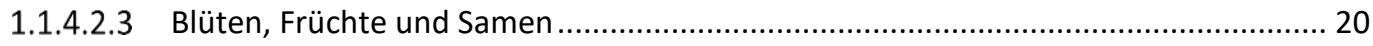

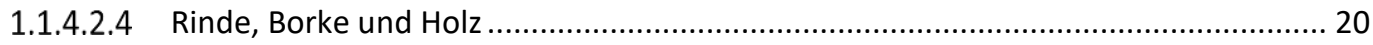

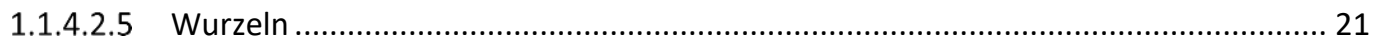

1.1.4.3 Waldbauliche und Ertragskundliche Behandlung ................................................... 21

1.1.4.3.1 Wachstum, Entwicklung und Ertrag .............................................................. 21

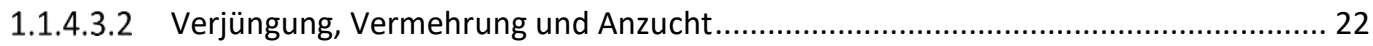

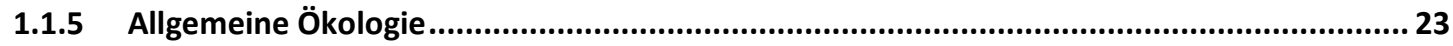

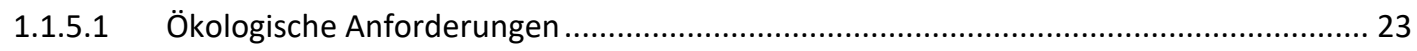

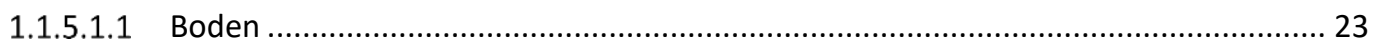

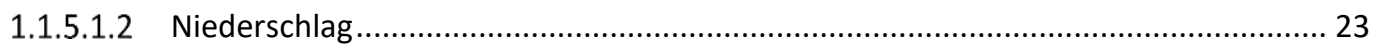

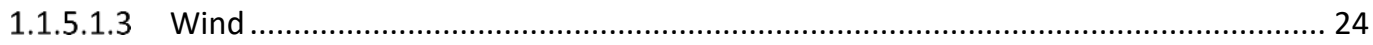

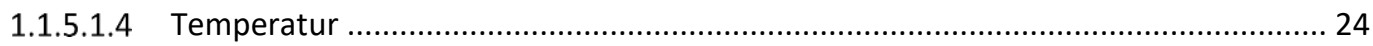

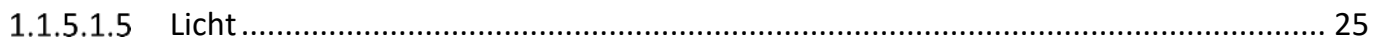

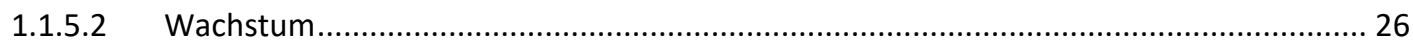

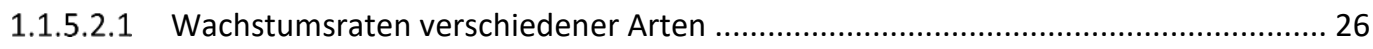

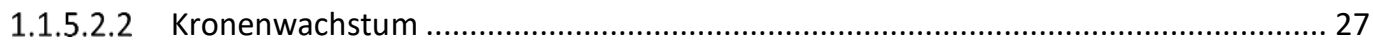

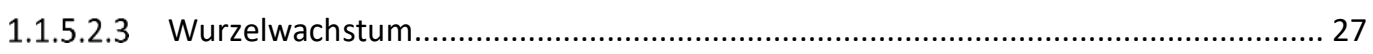

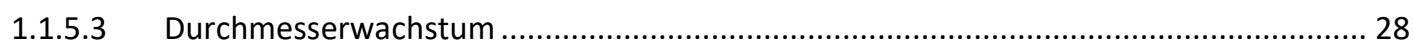

1.1.5.3.1 Durchmesserwachstum am Bsp. P.elongata ........................................................ 28

1.1.5.3.2 Durchmesserwachstum am Bsp. P.fortunei ......................................................... 28

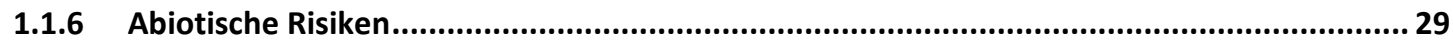

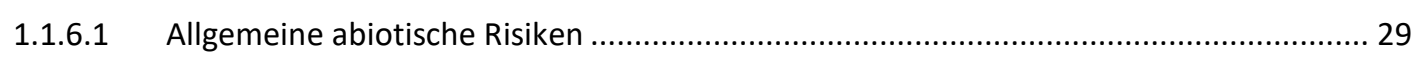

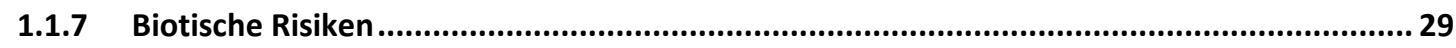

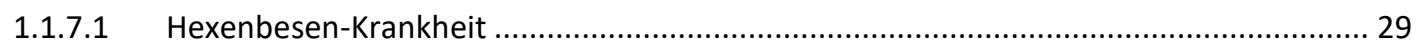

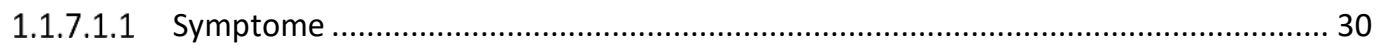

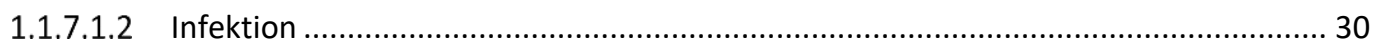

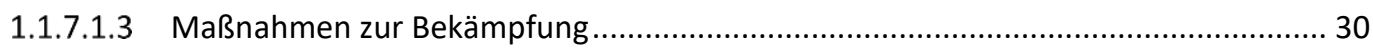

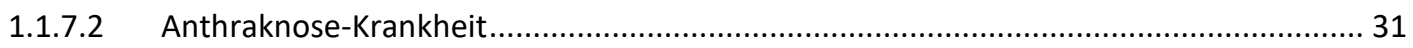

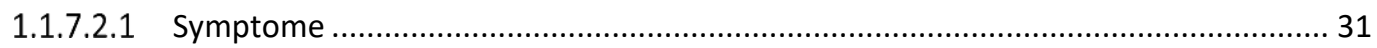

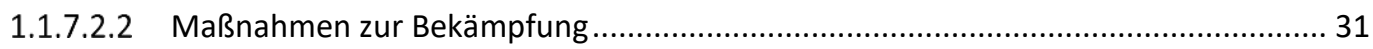

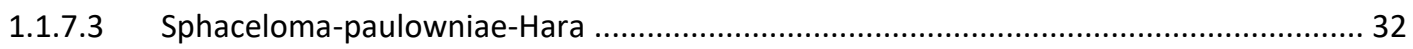

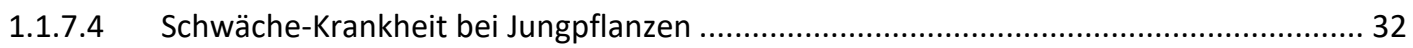

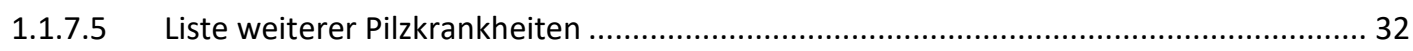

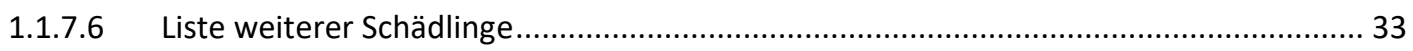

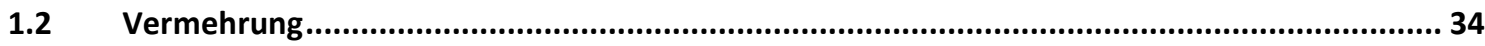

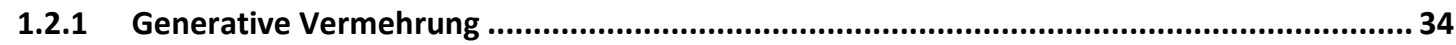

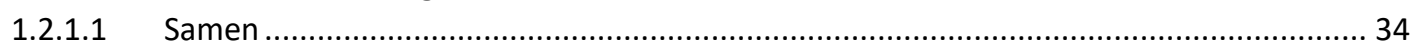

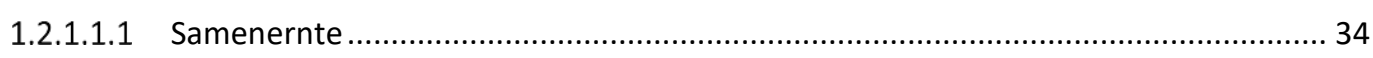

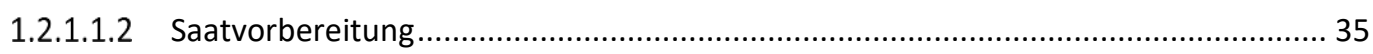

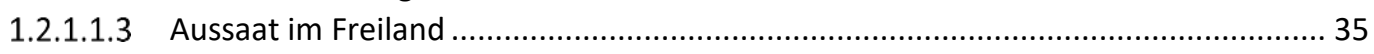

1.2.1.1.4 Anzucht in Pflanzcontainern ............................................................................. 35 
1.2.1.2 Setzlinge

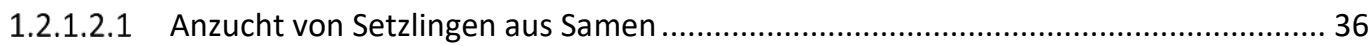

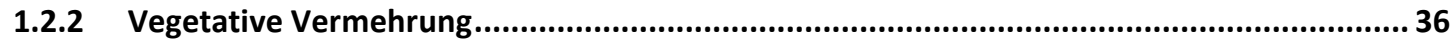

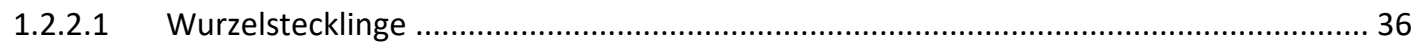

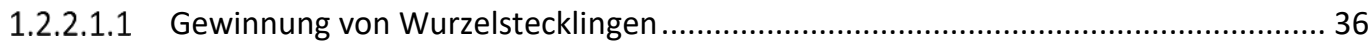

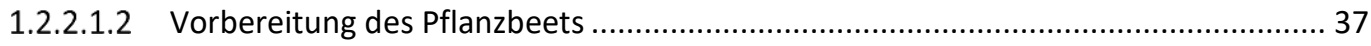

1.2.2.1.3 Anzucht von Setzlingen aus Wurzelstecklingen ................................................ 37

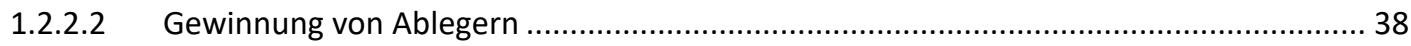

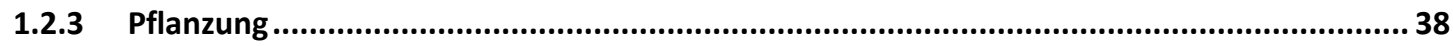

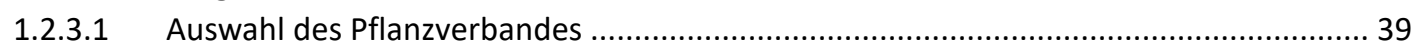

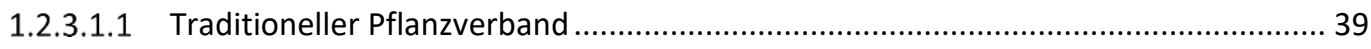

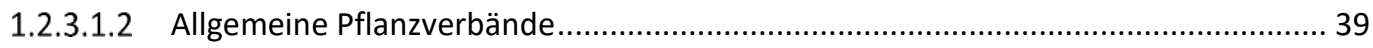

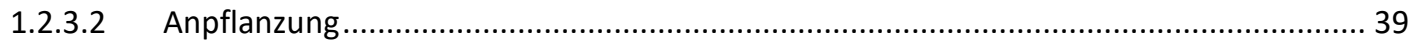

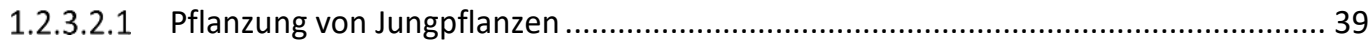

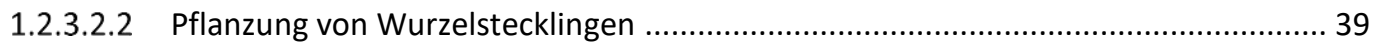

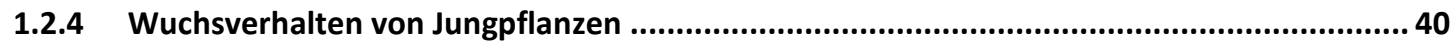

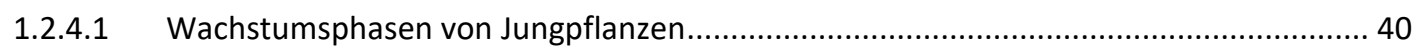

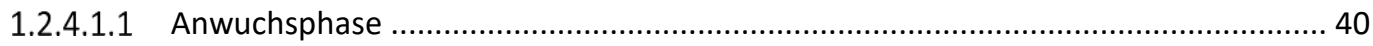

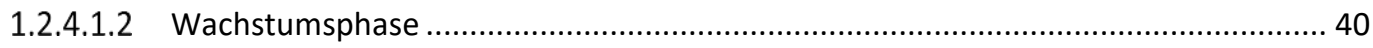

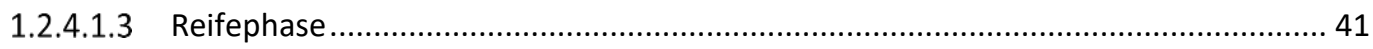

1.2.4.2 Regenerierung von minderwüchsigen Jungpflanzen .............................................. 41

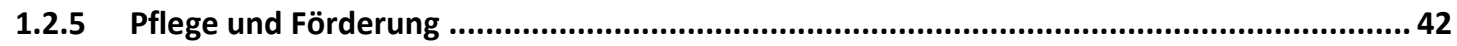

1.2.5.1 Allgemeine Pflege und Bewirtschaftung............................................................ 42

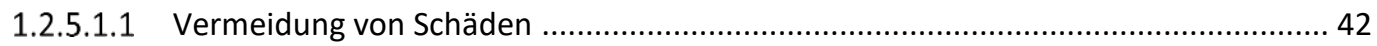

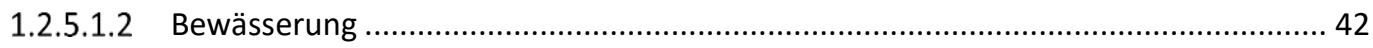

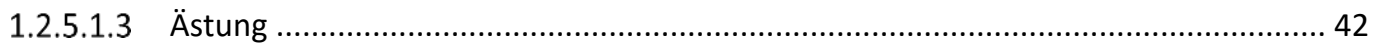

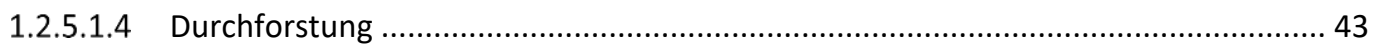

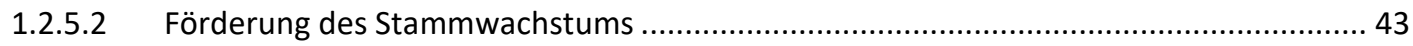

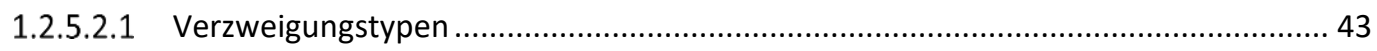

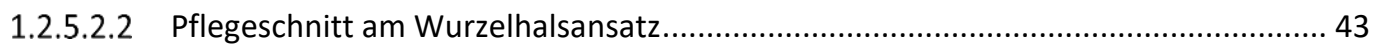

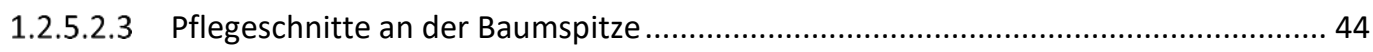

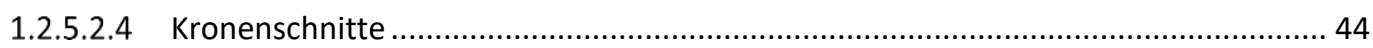

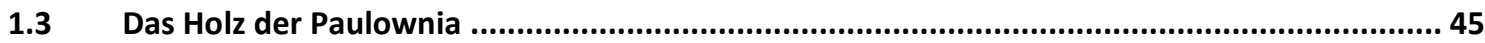

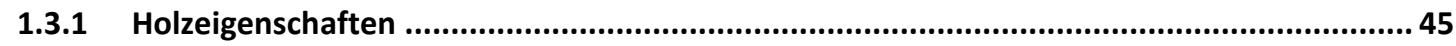

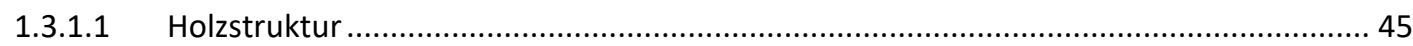

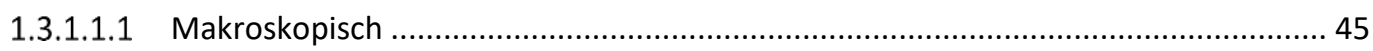

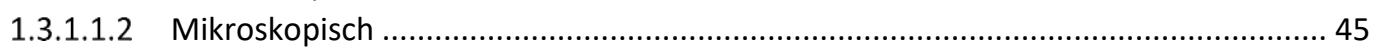

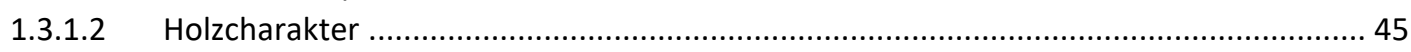

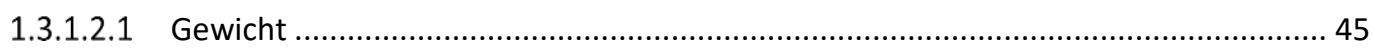

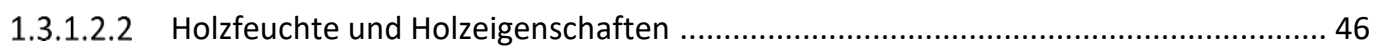

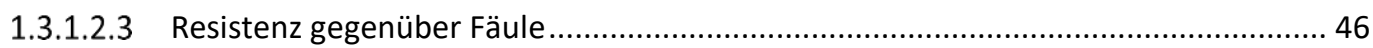

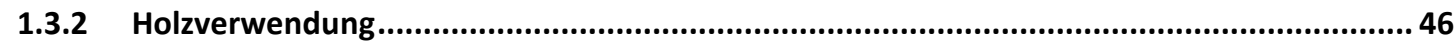

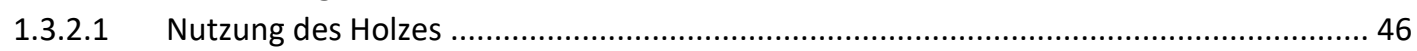

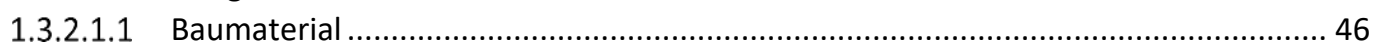

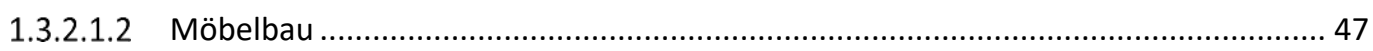

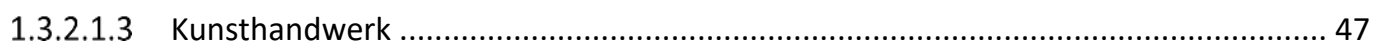

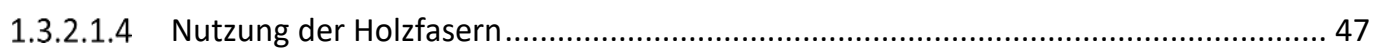

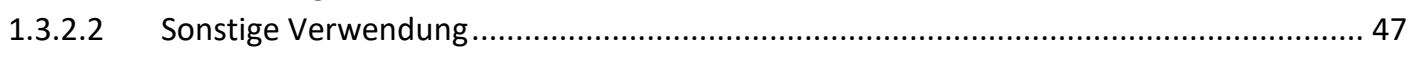

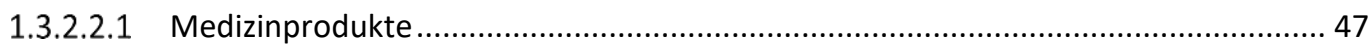

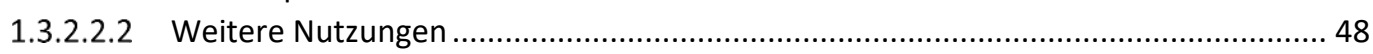


KAPITEL II

2. INVASIVITÄT, KURZUMTRIEB UND AGROFORST 49

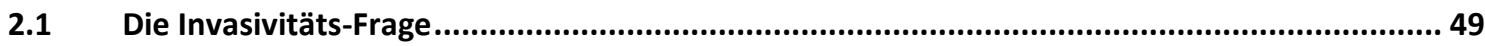

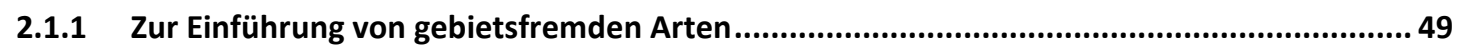

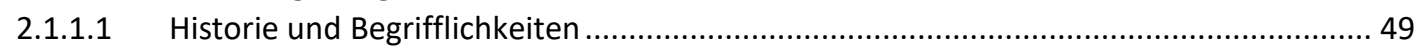

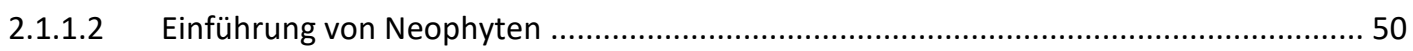

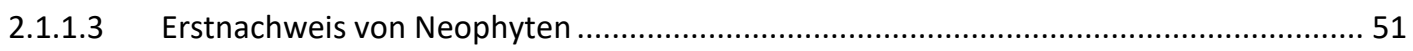

2.1.1.4 Ausbreitungspotential von Neophyten in Deutschland ............................................... 51

2.1.1.4.1 Zur Einführung von gebietsfremden Baumarten ................................................. 52

2.1.2 Rechtliche Rahmenbedingungen und Definitionen ....................................................53

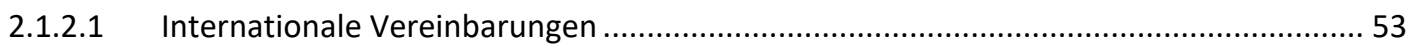

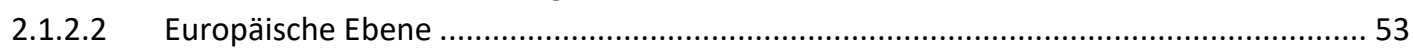

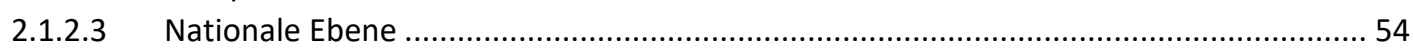

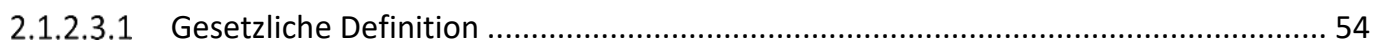

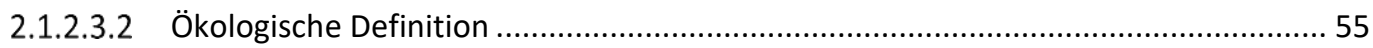

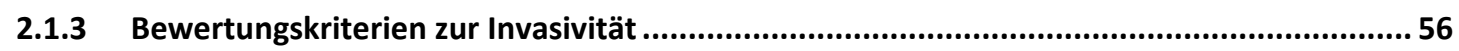

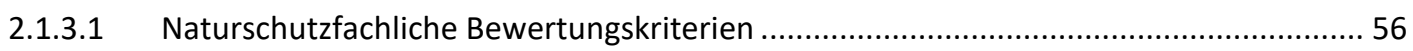

2.1.3.1.1 Kritik seitens der Forstwissenschaften ............................................................. 56

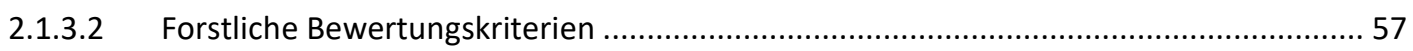

2.1.4 Bewertung der Invasivität von P.tomentosa .............................................................5 58

2.1.4.1 Naturschutzfachliche Invasivitätsbewertung ........................................................ 58

2.1.4.1.1 Hauptkriterien zur Invasivitätsbewertung ......................................................... 58

2.1.4.1.2 Zusatzkriterien zur Invasivitätsbewertung .......................................................... 59

2.1.4.2 Versuch einer forstlichen Invasivitätsbewertung .................................................... 59

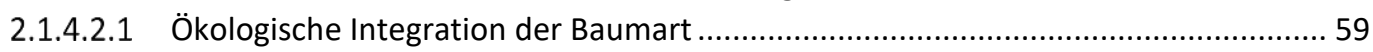

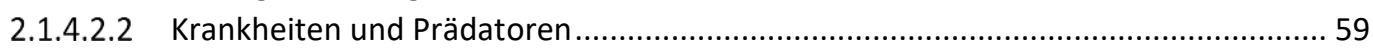

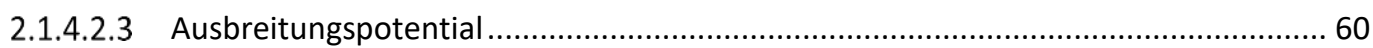

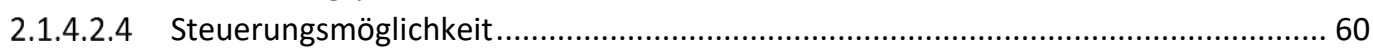

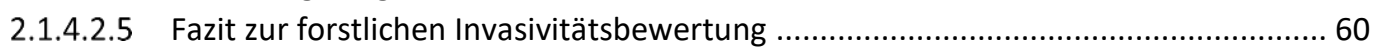

2.1.5 Maßnahmen im Umgang mit gebietsfremden Arten......................................................61

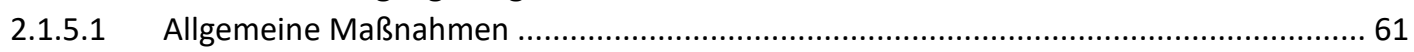

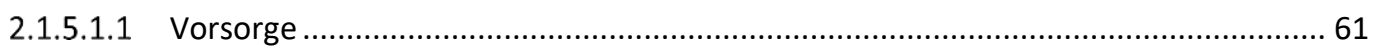

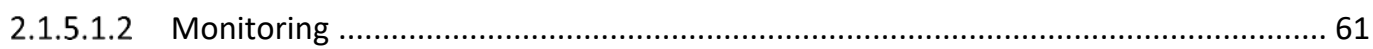

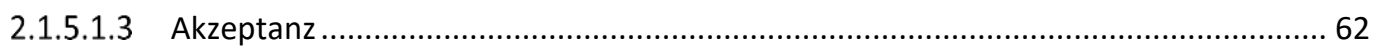

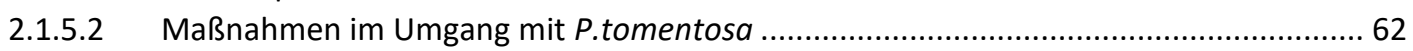

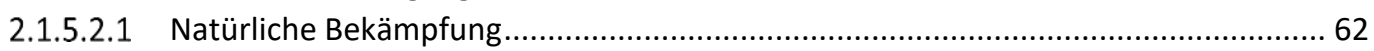

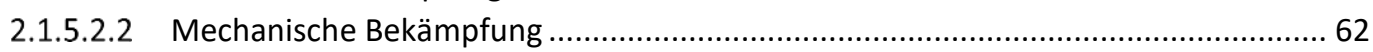

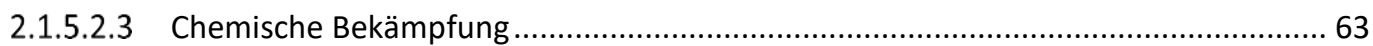

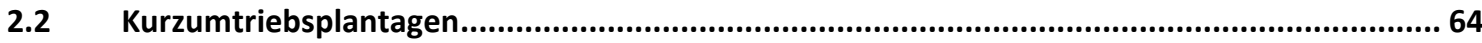

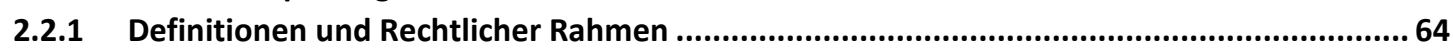

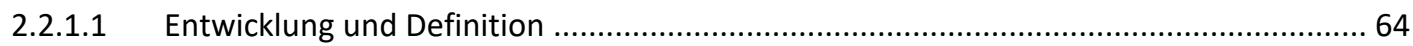

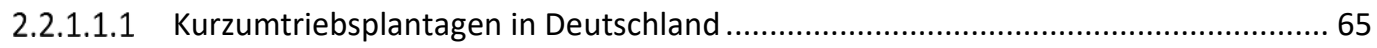

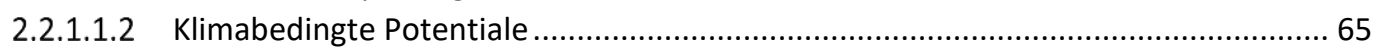

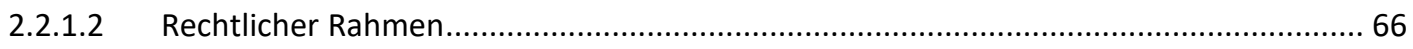

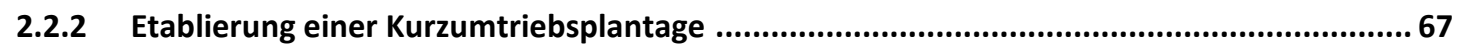

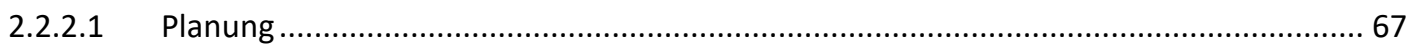

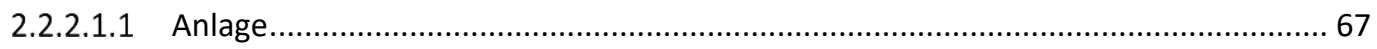

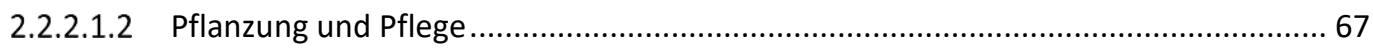

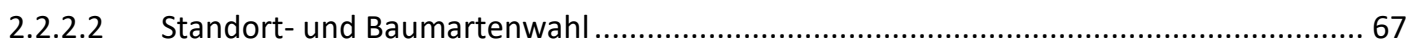

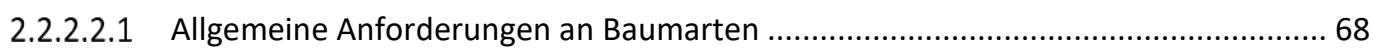


2.2.2.2.2 Standortbedingungen und Ziele des Bewirtschafters .......................................... 68

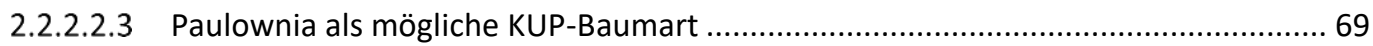

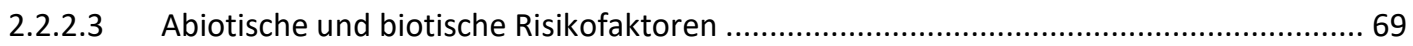

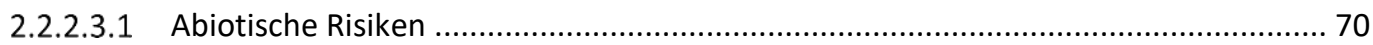

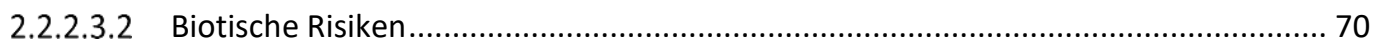

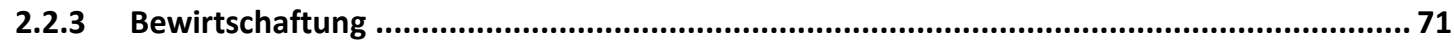

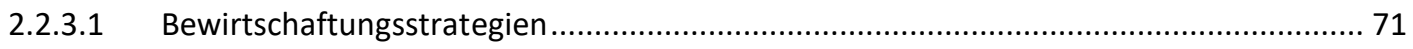

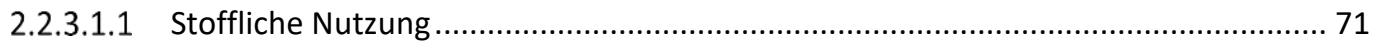

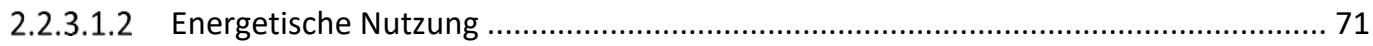

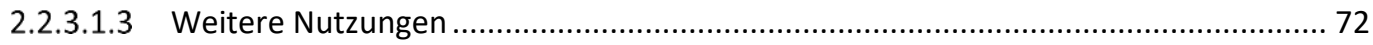

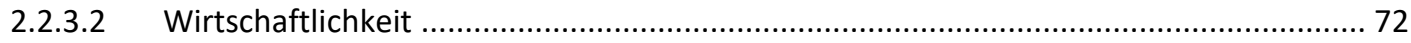

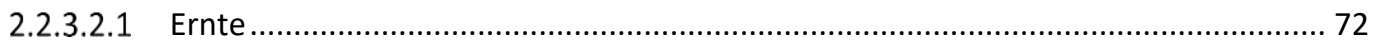

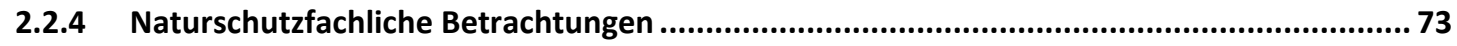

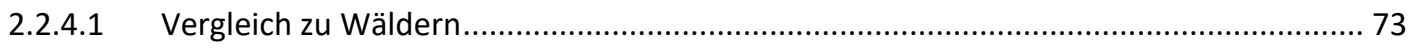

2.2.4.1.1 Natürlichkeitsgrad versus Bewirtschaftungsintensität ...................................... 73

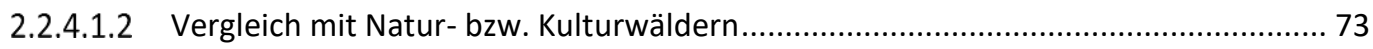

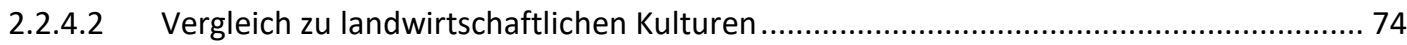

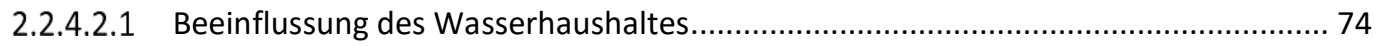

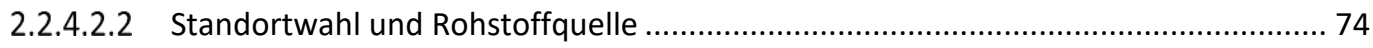

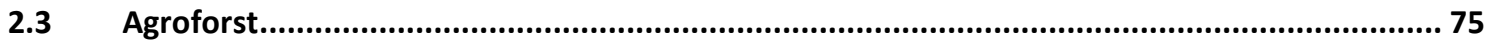

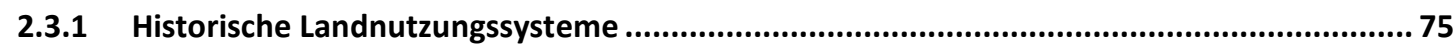

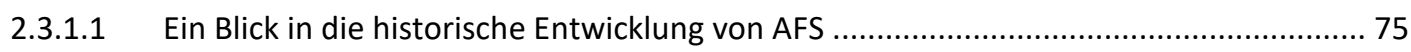

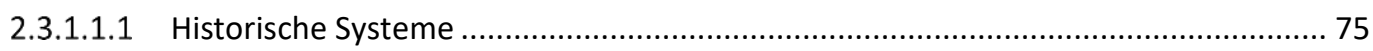

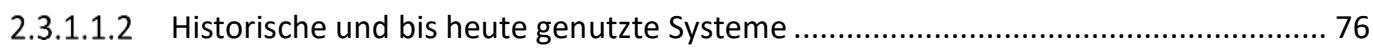

2.3.2 Definition und Rechtliche Rahmenbedingungen ........................................................... 76

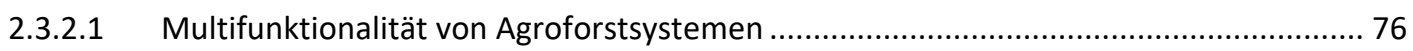

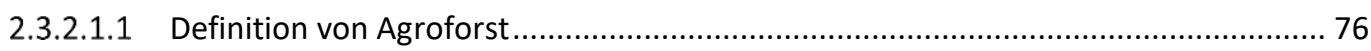

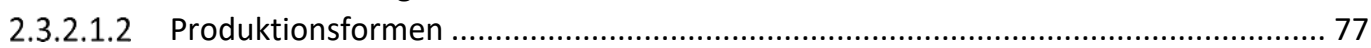

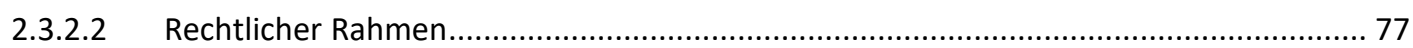

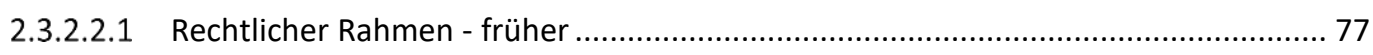

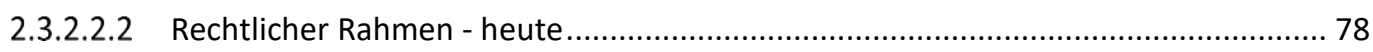

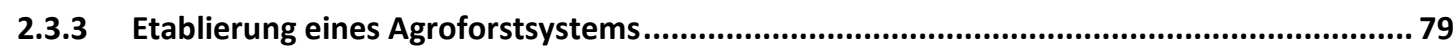

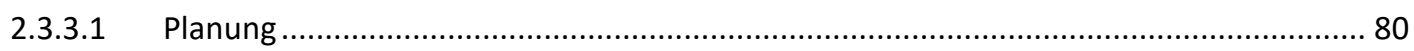

2.3.3.1.1 Besonderheiten von Agroforstsystemen mit Werthölzern .................................... 80

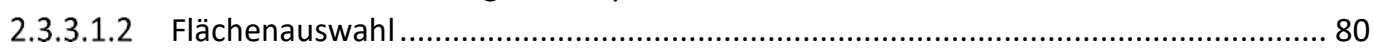

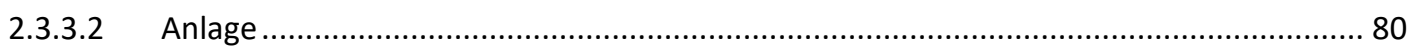

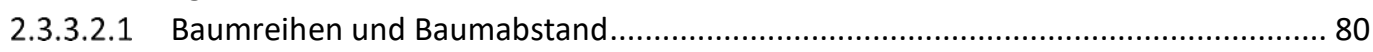

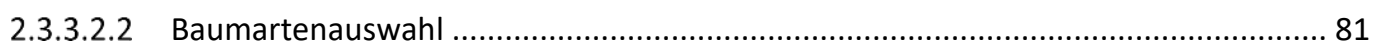

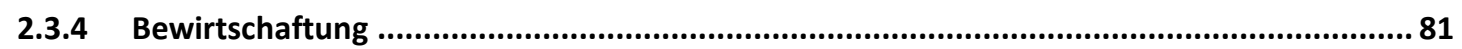

2.3.4.1 Ebenen der Bewirtschaftung ........................................................................... 81

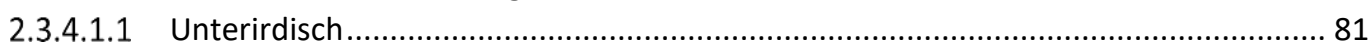

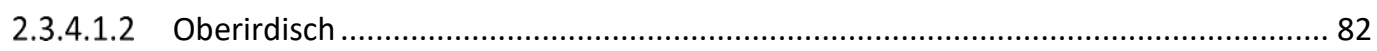

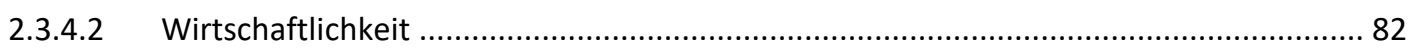

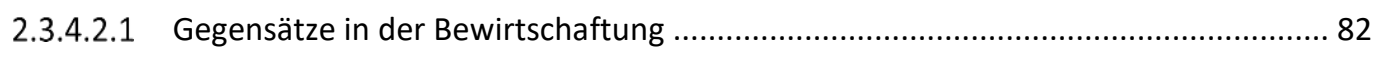

2.3.5 Naturschutzfachliche Betrachtungen .......................................................................83

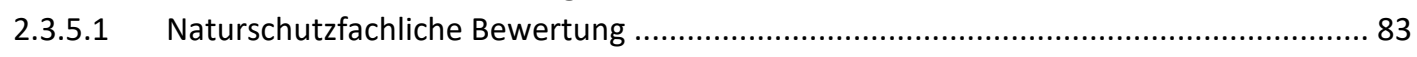

2.3.5.2 Möglichkeiten der Bewertung ........................................................................... 83

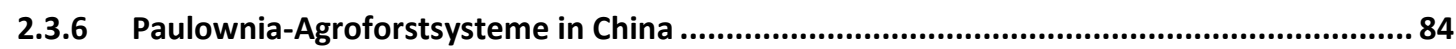

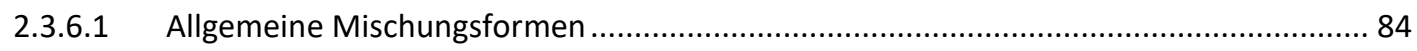

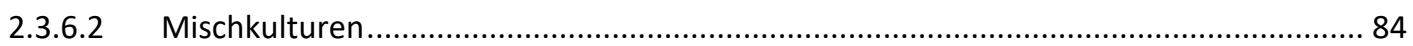

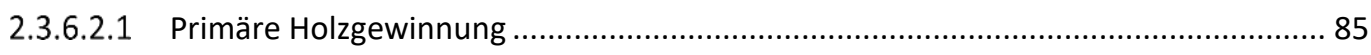

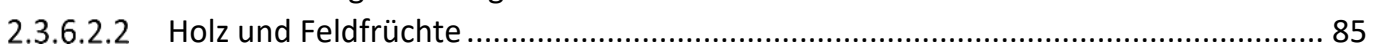

2.3.6.2.3 Primärer Feldfrüchteanbau ............................................................................. 85 
KAPITEL III

3. FREILAND- UND GEWÄCHSHAUSVERSUCHE

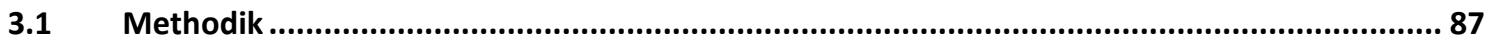

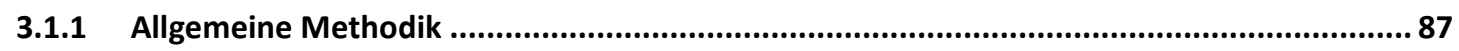

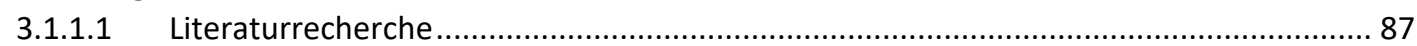

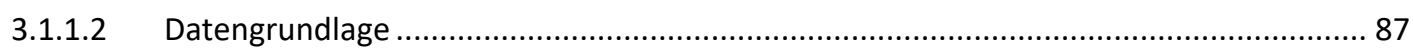

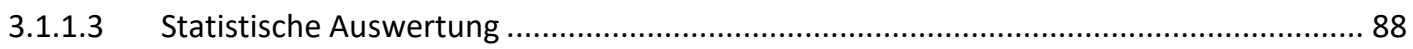

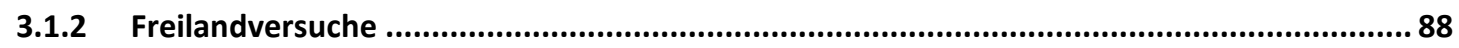

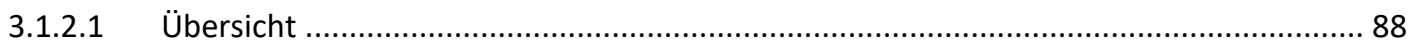

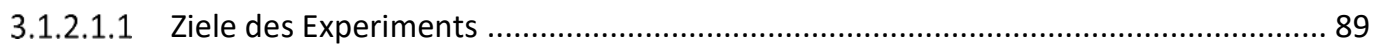

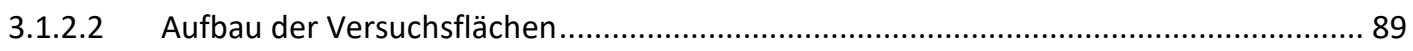

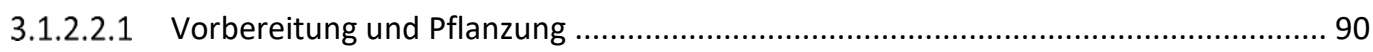

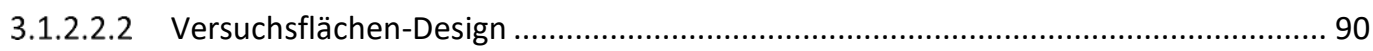

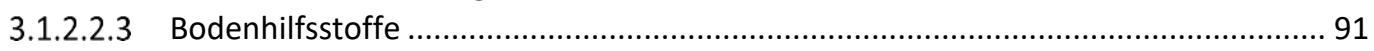

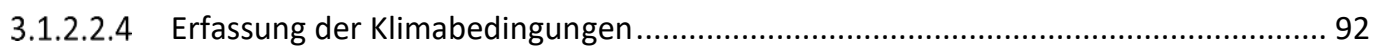

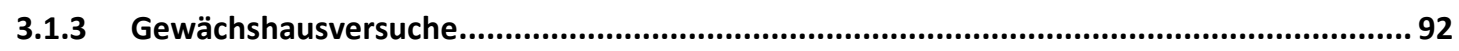

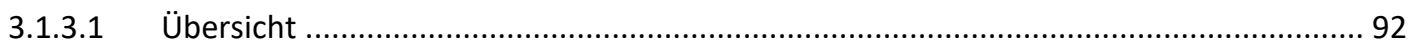

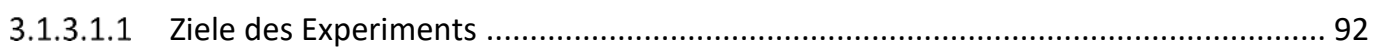

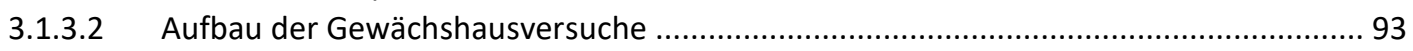

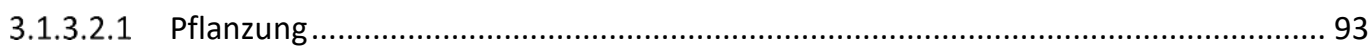

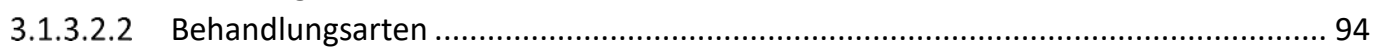

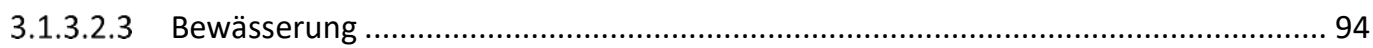

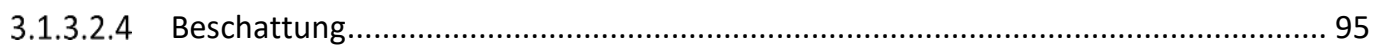

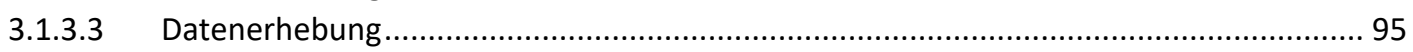

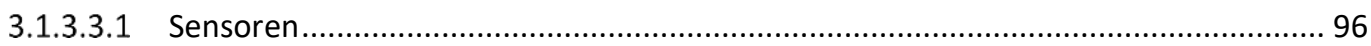

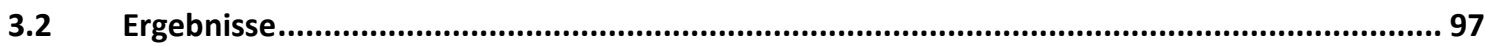

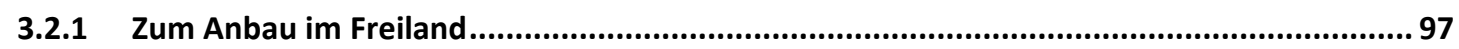

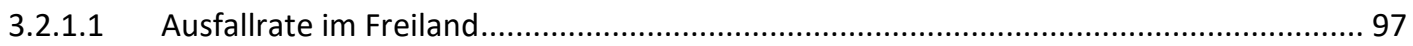

3.2.1.2 Witterungsverhältnisse auf den Versuchsflächen ........................................................ 99

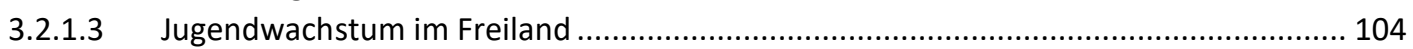

3.2.1.3.1 Jugendwachstum nach Behandlungsart......................................................... 104

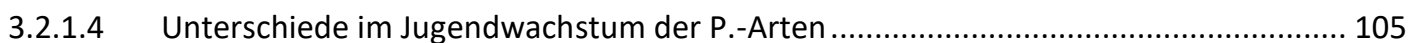

3.2.1.4.1 Brandenburger Versuchsfläche ...................................................................... 106

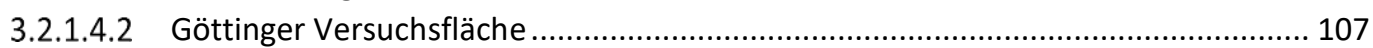

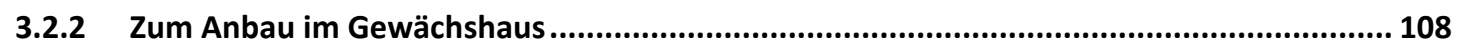

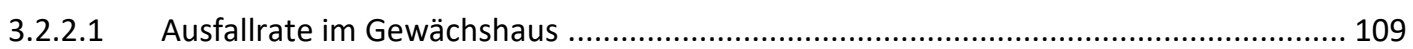

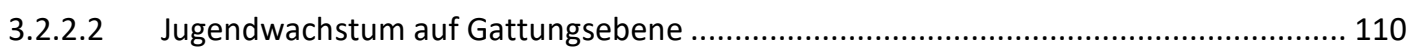

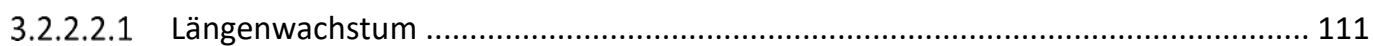

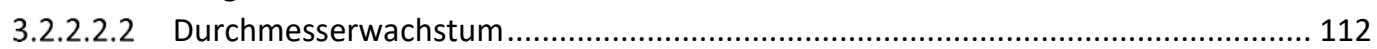

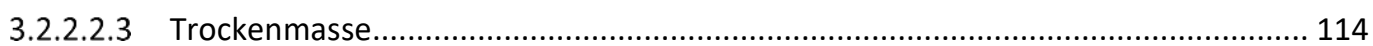

3.2.2.3 Jugendwachstum bei einem Vergleich der Arten ........................................................ 115

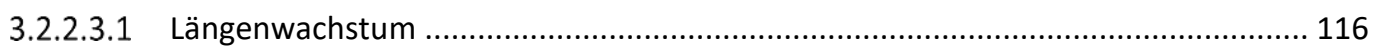

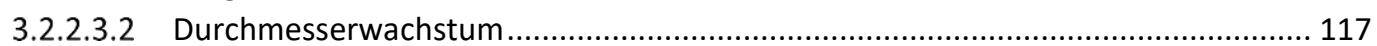

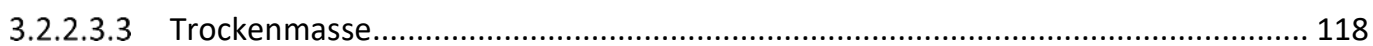

3.2.2.3.4 Blattfläche, Blattanzahl und Triebanzahl ......................................................... 120

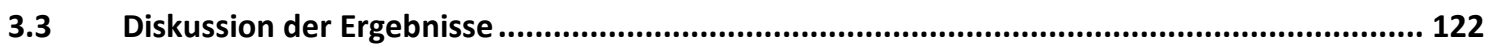

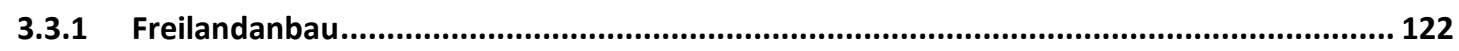

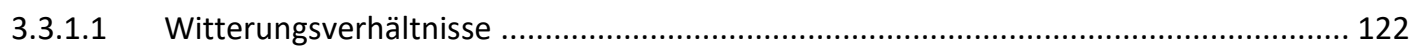

3.3.1.2 Bodenbearbeitung und Bodenverhältnisse …...................................................... 124 


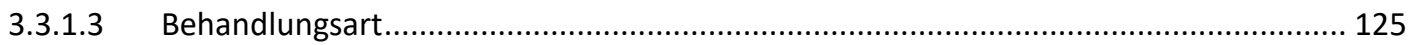

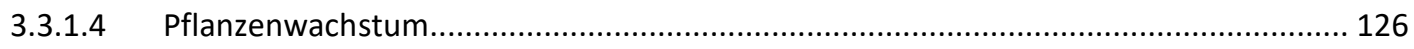

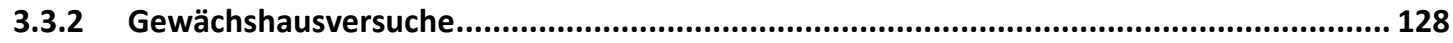

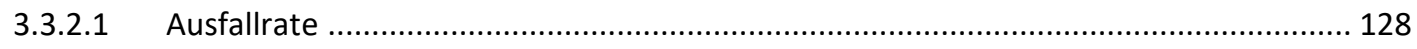

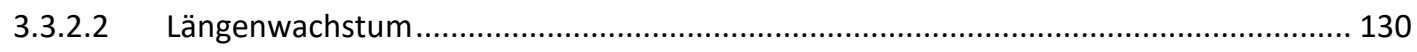

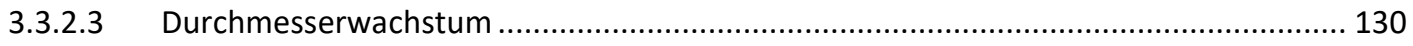

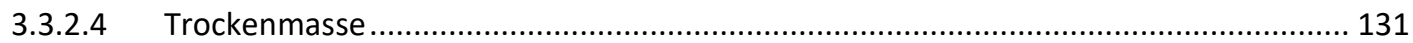

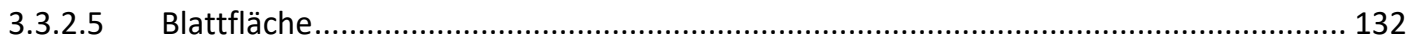

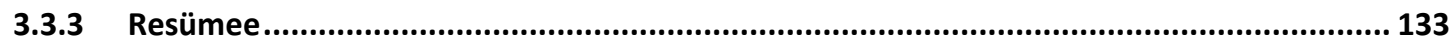

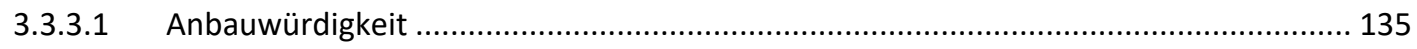

3.3.3.1.1 P.-Anbau auf Kurzumtriebsplantagen ........................................................ 135

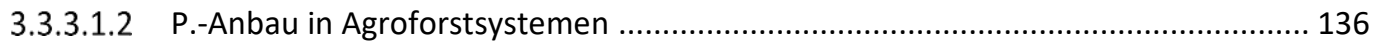

3.3.3.2 Anbauwürdigkeit vs. Invasivität....................................................................... 138

ANHANG ZUM KAPITEL I .................................................................................XXVI

ANHANG ZUM KAPITEL II ............................................................................. XXV

ANHANG ZUM KAPITEL III ............................................................................. XXVI

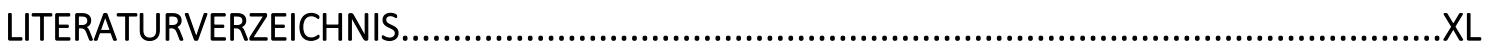

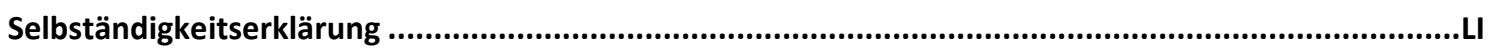

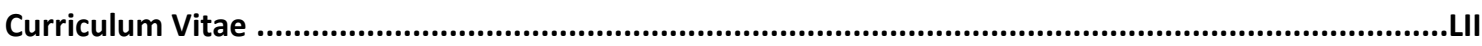




\section{Abbildungsverzeichnis}

ABB. 1: PAULOWNIA IMPERIALIS, BILDQUELLE:

HTTPS://DEACADEMIC.COM/PICTURES/DEWIKI/80/PAULOWNI_IMPERIALIS_SZ10.JPG I

ABB. 2: PAULOWNIA-BÄUME AUF DEN SÜDLICHEN SCHILLERWIESEN IN GÖTTINGEN.

ABB. 3: NATÜRLICHE VERBREITUNGSGEBIETE DER PAULOWNIA-ARTEN: P.ELONGATA, P.FORTUNEI, P.TOMENTOSA; VERÄNDERT NACH BARTON (2007).

ABB. 4: OBERES BILD ZEIGT EINJÄHRIGEN PAULOWNIA-WILDWUCHS IN DER GOßLER-STR.; UNTERES BILD ZEIGT EINJÄHRIGEN PAULOWNIA-WILDWUCHS AM INSTITUT FÜR DEMOKRATIEFORSCHUNG, IN DER WEENDER-LANDSTR., IN GÖTTINGEN.

ABB. 5: BLÜTENSTÄNDE UND SAMENKAPSELN EINER PAULOWNIA, IN DER GOßLER-STR., IN GÖTTINGEN.

ABB. 6. VERBREITUNGSGEBIET VON P.TOMENTOSA, VERÄNDERT NACH ZHU ET AL. (1991). 19

ABB. 7: BEGRIFFE ZUR EINTEILUNG DES ARTENBESTANDS, VERÄNDERT NACH NEOBIOTA (2020). 49

ABB. 8: PAULOWNIA-KUP MIT KÜNSTLICHER BEWÄSSERUNG (SCHWARZE LINIEN), BEI DER STADT PREMNITZ, LAND BRANDENBURG.

ABB. 9: VERGLEICH DER BEWIRTSCHAFTUNGSINTENSITÄT UND DES NATÜRLICHKEITSGRADES VON GEHÖLZÖKOSYSTEMEN, NACH SCHMIDT \& GEROLD (2008) ÜBERNOMMEN UND VERÄNDERT AUS SCHMIDT \& GLASER (2009).

ABB. 10: VERGLEICH VON WALD MIT AGROFORSTSYSTEMEN ZUR PRODUKTION VON WERTHÖLZERN, VERÄNDERT NACH CHALMIN \& MÖNDEL (2009).

ABB. 11: STANDORTE DER VERSUCHSFLÄCHEN, QUELLE: EIGENE DARSTELLUNG BASIEREND AUF GOOGLE MAPS.

ABB. 12: PAULOWNIA-PFLANZE UMRINGT VON BEGLEITWUCHS, AUF DER VERSUCHSFLÄCHE IN GÖTTINGEN.

ABB. 13: V.O.N.U. FUNKWETTERSTATIONEN AUF DER BRANDENBURGER-, FÖHRER- UND DER GÖTTINGER VERSUCHSFLÄCHE.

ABB. 14: GEOHUMUS GRANULAT, VERÄNDERT NACH BÜKER (2015).

ABB. 15: MYKORRHIZAPILZ-KONZENTRAT, VERÄNDERT NACH BÜKER (2015).

ABB. 16: SCHEMATISCHER AUFBAU DER PFLANZTISCHE, UNTERTEILUNG IN 3 P.-ARTEN (P.ELONGATA, P.FORTUNEI, P.TOMENTOSA), 3 LICHTSTUFEN (100 \%, $60 \%, 40 \%$ ) UND 3 WASSERSTUFEN (100\%, $60 \%, 40 \%)$.

ABB. 17: AUFBAU DER PFLANZTISCHE IM GEWÄCHSHAUS; LINKS IM VORDERGRUND DER TISCH MIT 100 $\%$ LICHT, GEFOLGT VOM 60 \% LICHT- UND 40 \% LICHT-TISCH UND ANSCHLIEßEND NOCH EINMAL WIEDERHOLT.

ABB. 18: PFLANZTISCHE IM GEWÄCHSHAUS - ABGEERNTET.

ABB. 19: GESAMT-AUSFALLRATE DER BAUMARTEN (IN \%) AUF DEN VERSUCHSFLÄCHEN BRANDENBURG (BB) UND GÖTTINGEN (GÖ), UNTERTEILT IN DIE VEGETATIONSPERIODEN DER JAHRE 2015 BIS 2017.

ABB. 20: OBERE GRAFIK BESCHREIBT DIE EVAPOTRANSPIRATION DER JAHRE 2015, 2016 UND 2017 DER VERSUCHSFLÄCHE BRANDENBURG (BB): VGSLMM = REALE EVAPOTRANSPIRATION VON GRAS ÜBER SANDIGEM LEHM (AMBAV), BF10\% = BODENFEUCHTE UNTER GRAS BEI SANDIGEM LEHM IN EINER TIEFE ZWISCHEN $0-10 \mathrm{CM}$, BFGSL\% = GIBT DIE NUTZBARE FELDKAPAZITÄT AN UND BESCHREIBT DIE BODENFEUCHTE UNTER GRAS BEI SANDIGEM LEHM IN EINER TIEFE ZWISCHEN 0 60 CM; UNTERE GRAFIK: MONATLICHE NIEDERSCHLAGSWERTE DER VERSUCHSFLÄCHE BRANDENBURG IN 1 ZU 10 MM (BLAUE BALKEN = VEGETATIONSPERIODE), MONATLICHE DURCHSCHNITTSTEMPERATUR IN $C^{\circ}$ (VIOLETTE LINIE); QUELLE: EIGENE DARSTELLUNG AUF BASIS VON DWD-DATEN.

ABB. 21: OBERE GRAFIK BESCHREIBT DIE EVAPOTRANSPIRATION DER JAHRE 2015 BIS 2017 DER VERSUCHSFLÄCHE GÖTTINGEN (GÖ): VGSLMM = REALE EVAPOTRANSPIRATION VON GRAS ÜBER SANDIGEM LEHM (AMBAV), BF10\% = BODENFEUCHTE UNTER GRAS BEI SANDIGEM LEHM IN EINER TIEFE ZWISCHEN 0 - $10 \mathrm{CM}$, BFGSL\% = GIBT DIE NUTZBARE FELDKAPAZITÄT AN UND BESCHREIBT DIE BODENFEUCHTE UNTER GRAS BEI SANDIGEM LEHM IN EINER TIEFE ZWISCHEN 0 - 60 CM; 
UNTERE GRAFIK: MONATLICHE NIEDERSCHLAGSWERTE DER VERSUCHSFLÄCHE GÖTTINGEN IN 1 ZU 10 MM (BLAUE BALKEN = VEGETATIONSPERIODE), MONATLICHE

DURCHSCHNITTSTEMPERATUR IN C ${ }^{\circ}$ (VIOLETTE LINIE); QUELLE: EIGENE DARSTELLUNG AUF BASIS VON DWD-DATEN.

ABB. 22: HÖHENWACHSTUM DER PAULOWNIA-PFLANZEN (IN CM) FÜR DIE VEGETATIONSPERIODEN (VP) 2015, 2016 UND 2017, FÜR DIE VERSUCHSFLÄCHEN BRANDENBURG (BB) UND GÖTTINGEN (GÖ); UNTERTEILT NACH BEHANDLUNGSARTEN: N = UNBEHANDELT (NULLFLÄCHE), G = GEOHUMUS, $M=$ MYKORRHIZIERUNG, GM = GEOHUMUS + MYKORRHIZIERUNG.

ABB. 23: HÖHENWACHSTUM DER PAULOWNIA-ARTEN (IN CM) AUF DER VERSUCHSFLÄCHE IN BRANDENBURG; PE = P.ELONGATA; PF = P.FORTUNEI; PT = P.TOMENTOSA FÜR DIE VEGETATIONSPERIODEN (VP) 2015, 2016 UND 2017 UNTERTEILT NACH BEHANDLUNGSARTEN: $N=$ UNBEHANDELT (NULLFLÄCHE), G = GEOHUMUS, M = MYKORRHIZIERUNG, GM = GEOHUMUS + MYKORRHIZIERUNG.

ABB. 24: HÖHENWACHSTUM DER PAULOWNIA-ARTEN (IN CM) AUF DER VERSUCHSFLÄCHE IN GÖTTINGEN; PE = P.ELONGATA; PF = P.FORTUNEI; PT = P.TOMENTOSA FÜR DIE VEGETATIONSPERIODEN (VP) 2015, 2016 UND 2017 UNTERTEILT NACH BEHANDLUNGSARTEN: $N=$ UNBEHANDELT (NULLFLÄCHE), G = GEOHUMUS, M = MYKORRHIZIERUNG, GM = GEOHUMUS + MYKORRHIZIERUNG.

ABB. 25: VERGLEICH DES LÄNGENWACHSTUMS ALLER PAULOWNIA-PFLANZEN ZWISCHEN DER ERSTEN UND ZWEITEN VEGETATIONSPERIODE (VP). LINKE GRAFIK BESCHREIBT UNTERSCHIEDLICHE LICHTSTUFEN (100LI, 60LI, 40LI) BEI GLEICHBLEIBENDER 100 \% BEWÄSSERUNG. RECHTE GRAFIK BESCHREIBT DIE LICHTSTUFE 40 \% BEI ABNEHMENDER BEWÄSSERUNG (100WA, 60WA, 40WA). 111

ABB. 26: VERGLEICH DES LÄNGENWACHSTUMS ALLER PAULOWNIA-PFLANZEN ZWISCHEN DER ERSTEN UND ZWEITEN VEGETATIONS-PERIODE (VP), BEI ABNEHMENDEN LICHTSTUFEN (100LI, 60LI, 40LI) UND GLEICHBLEIBENDER $40 \%$ BEWÄSSERUNG.

ABB. 27: VERGLEICH DES WURZELHALSDURCHMESSERS ZWISCHEN ERSTER UND ZWEITER VEGETATIONSPERIODE (VP). LINKE GRAFIK BESCHREIBT UNTERSCHIEDLICHE LICHTSTUFEN (100LI, 60LI, 40LI) BEI GLEICHBLEIBENDER $100 \%$ BEWÄSSERUNG. RECHTE GRAFIK BESCHREIBT DIE $40 \%$ LICHTSTUFE BEI ABNEHMENDER BEWÄSSERUNG (100WA, 60WA, 40WA).

ABB. 28: VERGLEICH DES WURZELHALSDURCHMESSERS ZWISCHEN ERSTER UND ZWEITER VEGETATIONSPERIODE (VP) BEI GLEICHBLEIBENDER $100 \%$ LICHTSTUFE UND ABNEHMENDER BEWÄSSERUNG (100WA, 60WA, 40WA).

ABB. 29: VERHÄLTNIS VON HÖHE ZU DURCHMESSER IN ERSTER (BLAU) UND ZWEITER (GRÜN) VEGETATIONSPERIODE (VP).

ABB. 30: VERGLEICH DES TROCKENGEWICHTS (IN GRAMM) ALLER PAULOWNIA-PFLANZEN ZWISCHEN DER ERSTEN UND ZWEITEN VEGETATIONSPERIODE (VP). LINKE GRAFIK MIT $100 \%$ LICHT, RECHTE GRAFIK MIT 40 \% LICHT BEI JEWEILS UNTERSCHIEDLICHER BEWÄSSERUNG (100WA, 60WA, 40WA).

ABB. 31: VERGLEICH DES TROCKENGEWICHTS ALLER PAULOWNIA-PFLANZEN ZWISCHEN DER ERSTEN UND ZWEITEN VEGETATIONSPERIODE (VP). BEI UNTERSCHIEDLICHEN LICHTSTUFEN (100LI, 60LI, 40LI) UND $40 \%$ BEWÄSSERUNG.

ABB. 32: VERHÄLTNIS VON HÖHE ZU GEWICHT ALLER PAULOWNIA-PFLANZEN IM VERGLEICH VON ERSTER (GRÜN) ZU ZWEITER (BLAU) VEGETATIONSPERIODE (VP).

ABB. 33: VERGLEICH DES LÄNGENWACHSTUMS DER PAULOWNIA-ARTEN BEI DER 40 \% LICHTSTUFE UND UNTERSCHIEDLICHER WASSERVERSORGUNG (100WA, 60WA, 40WA). LINKE GRAFIK BESCHREIBT ERSTE UND RECHTE GRAFIK DIE ZWEITE VEGETATIONSPERIODE.

ABB. 34: VERGLEICH DES LÄNGENWACHSTUMS DER PAULOWNIA-ARTEN BEI $100 \%$ BEWÄSSERUNG ABER UNTERSCHIEDLICHEN LICHTSTUFEN (100LI, 60LI, 40LI). LINKE GRAFIK BESCHREIBT ERSTE UND RECHTE GRAFIK DIE ZWEITE VEGETATIONSPERIODE.

ABB. 35: VERGLEICH DES WURZELHALSDURCHMESSERS DER P.-ARTEN ZWISCHEN ERSTER (LINKE GRAFIK) UND ZWEITER (RECHTE GRAFIK) VEGETATIONSPERIODE. BEIDE GRAFIKEN ZEIGEN DIE $40 \%$ LICHTSTUFE BEI UNTERSCHIEDLICHER BEWÄSSERUNG (100WA, 60WA, 40WA). 
ABB. 36: VERGLEICH DES WURZELHALSDURCHMESSERS DER P.-ARTEN ZWISCHEN ERSTER (LINKE GRAFIK) UND ZWEITER (RECHTE GRAFIK) VEGETATIONSPERIODE. BEIDE GRAFIKEN ZEIGEN DIE 100 \% BEWÄSSERUNGSSTUFE BEI UNTERSCHIEDLICHER LICHTZUFUHR (100LI, 60LI, 40LI). 118

ABB. 37: VERGLEICH DES TROCKENGEWICHTS DER PAULOWNIA-ARTEN ZWISCHEN DER ERSTEN (LINKE GRAFIK) UND ZWEITEN (RECHTE GRAFIK) VEGETATIONSPERIODE. JEWEILS MIT $100 \%$ BEWÄSSERUNG BEI UNTERSCHIEDLICHER BELICHTUNG (100LI, 60LI, 40LI).

ABB. 38: VERGLEICH DES TROCKENGEWICHTS DER PAULOWNIA-ARTEN ZWISCHEN DER ERSTEN (LINKE GRAFIK) UND ZWEITEN (RECHTE GRAFIK) VEGETATIONSPERIODE. JEWEILS MIT $100 \%$ LICHT BEI UNTERSCHIEDLICHER BEWÄSSERUNG (100WA, 60WA, 40WA).

ABB. 39: VERGLEICH DES TROCKENGEWICHTS DER PAULOWNIA-ARTEN ZWISCHEN DER ERSTEN (LINKE GRAFIK) UND ZWEITEN (RECHTE GRAFIK) VEGETATIONSPERIODE. JEWEILS MIT $40 \%$ BEWÄSSERUNG BEI UNTERSCHIEDLICHER LICHTZUFUHR (100LI, 60LI, 40LI).

ABB. 40: ANZAHL BLÄTTER PRO PFLANZE UNTERTEILT NACH BEHANDLUNGSSTUFEN (LICHT UND WASSER) UND AUFGESCHLÜSSELT NACH PAULOWNIA-ARTEN.

ABB. 41: BLATTFLÄCHE PRO PFLANZE IN CM² UNTERTEILT NACH BEHANDLUNGSSTUFEN (LICHT UND WASSER) UND AUFGESCHLÜSSELT NACH PAULOWNIA-ARTEN.

ABB. 42: DURCHSCHNITTLICHE ANZAHL VON TRIEBEN PRO PFLANZE UNTERTEILT NACH BEHANDLUNGSSTUFEN (LICHT UND WASSER) UND AUFGESCHLÜSSELT NACH PAULOWNIA-ARTEN.

ABB. 43: VITALITÄTSSTUFEN NACH VISNJIC (2006), V.L.N.R.: STUFE 1 = VITALE PFLANZE, STUFE 2 = MÄßIG VITALE PFLANZE, STUFE 3 = GERING VITALE PFLANZE, STUFE 4 = VERTROCKNETE PFLANZE; VERÄNDERT NACH BÜKER (2015).

ABB. 44: WELKEN DER PAULOWNIA-BLÄTTER DURCH TROCKENSTRESS, AUF DER VERSUCHSFLÄCHE IN BRANDENBURG.

ABB. 45: PAULOWNIA-PFLANZE MIT EINER HÖHE VON 45 CM IM ALTER VON 6 MONATEN AUF DER VERSUCHSFLÄCHE IN BRANDENBURG.

ABB. 46: ABGEERNTETE PAULOWNIA-STÄNGEL AUS DEM GEWÄCHSHAUS; ERKENNBAR DER RÖHRENQUERSCHNITT.

ABB. 47: JAHRESTRIEB EINER P.ELONGATA IN EINEM PRIVATEN VORGARTEN IN DER LEONARD-NELSONSTR., IN GÖTTINGEN.

ABB. 48: NATURSCHUTZFACHLICHE INVASIVITÄTSBEWERTUNG VON P.TOMENTOSA, ENTNOMMEN UND VERÄNDERT NACH NEHRING ET AL. (2013).

ABB. 49: INFORMATIONSBLÄTTER ZUM BODENHILFSSTOFF: GEOHUMUS.

$X X X V$

ABB. 50: INFORMATIONSBLATT ZUM MYKORRHIZAPILZ-KONZENTRAT.

$X X X V I$

ABB. 51: KARTEN ZU DEN VERSUCHSFLÄCHEN IN BRANDENBURG, FÖHR UND GÖTTINGEN.

$X X X V I I$ $X X X V I I$

\section{Tabellenverzeichnis}

TAB. 1: VERGLEICH DER PAULOWNIA ARTEN ANHAND VON VERBREITUNGSGEBIET, TEMPERATUR, NIEDERSCHLAG UND BODENVERHÄLTNISSEN, VERÄNDERT NACH ZHU ET AL. (1986), S. 22.

TAB. 2: VERGLEICH DES WACHSTUMS VON 5-JÄHRIGEN BÄUMEN DER ARTEN P.ELONGATA,

P.TOMENTOSA, P.FORTUNEI, P.GLABRATA AUS DER WU GUENG REGION (SÜDLICH DES YANGTZE-

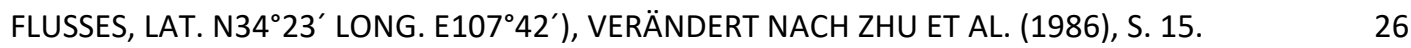

TAB. 3: AKTUELLE ANZAHL NEOBIOTA IN DEUTSCHLAND, STAND 2015.

TAB. 4: WASSERMENGEN DER DREI BEWÄSSERUNGSSTUFEN. 94

TAB. 5: VERGLEICH DER MONATLICHEN TEMPERATUR- UND NIEDERSCHLAGSENTWICKLUNG, DER JAHRE 2015 BIS 2017 AUF DEN VERSUCHSFLÄCHEN IN BRANDENBURG (BB) UND GÖTTINGEN (GÖ), MIT DEM LANGJÄHRIGEN MITTELWERT DER JAHRE 1981 - 2010. QUELLE: EIGENE DARSTELLUNG AUF BASIS VON DWD-DATEN.

TAB. 6: TABELLARISCHER ÜBERBLICK ÜBER DIE BODENBESCHAFFENHEIT AUF DEN VERSUCHSFLÄCHEN IN BRANDENBURG, FÖHR UND GÖTTINGEN. QUELLE: EIGENE DARSTELLUNG MITTELS GEOVIEWER UND DWD-DATEN. 
TAB. 7: AUSFALLRATE DER PAULOWNIA-PFLANZEN (IN \%) AUFGESCHLÜSSELT NACH BEHANDLUNGSSTUFEN (LICHT UND WASSER) UND UNTERTEILT IN ERSTE UND ZWEITE VEGETATIONSPERIODE UND NACH ARTEN (P.ELONGATA (PE), P.FORTUNEI (PF), P.TOMENTOSA (PT)).

TAB. 8: BESTIMMUNGSSCHLÜSSEL DER PAULOWNIA ARTEN IM DIREKTEN VERGLEICH, VERÄNDERT NACH ZHU ET AL. (1986), S. 9.

TAB. 9: GESAMTÜBERBLICK ÜBER DIE ARTEN UND HYBRIDEN DIE VON PAULOWNIA ÜBER INTERNATIONAL PLANT NAMES INDEX (2020) BEKANNT SIND:

TAB. 10: TABELLE ÜBER PILZE UND ARTHROPODEN DIE PAULOWNIA NACHWEISLICH BESIEDELN. DIE FOLGENDE ÜBERSICHT WURDE ÜBERNOMMEN UND VERÄNDERT NACH HAO ET AL. (2004): XXVIII

TAB. 11: BEWERTUNG DER INVASIVITÄT VON BAUMARTEN AUS SICHT DER FORSTWISSENSCHAFTEN, ENTNOMMEN UND VERÄNDERT NACH VOR ET AL. (2015).

TAB. 12: TABELLARISCHE AUFLISTUNG DER HOLZEIGENSCHAFTEN VON VERSCHIEDENEN PAULOWNIAARTEN, ÜBERNOMMEN UND VERÄNDERT NACH ZHU ET AL. (1986), S. 59.

TAB. 13: TABELLARISCHE AUFLISTUNG DER SIGNIFIKANZEN (MITTELWERTVERGLEICHE) ZWISCHEN DEN VERSCHIEDENEN BAUMARTEN UND BEHANDLUNGSARTEN, UNTERTEILT NACH DEN VEGETATIONSPERIODEN 2015, 2016 UND 2017 UND NACH DEN STANDORTEN BRANDENBURG (BB) UND GÖTTINGEN (GÖ). LEERE FELDER ZEIGEN KEINE SIGNIFIKANZEN. GRAUE FELDER MARKIEREN FEHLENDE DATEN WEGEN DER HOHEN AUSFALLRATEN.

\section{Abkürzungsverzeichnis}

\begin{tabular}{|l|l|}
\hline AFS & Agroforstsystem \\
\hline APA & American Paulownia Association \\
\hline BfN & Bundesamt für Naturschutz \\
\hline BHD & Brusthöhendurchmesser, gemessener Baumdurchmesser auf 1,3 m Höhe \\
\hline BNatSchG & Bundesnaturschutzgesetz \\
\hline BSc & Bachelor of Science \\
\hline BWaldG & Bundeswaldgesetz \\
\hline CO ${ }^{2}$ & Kohlendioxid \\
\hline EU & Europäische Union \\
\hline ha & Hektar \\
\hline KUP & Kurzumtriebsplantage \\
\hline Ix & Lux = Einheit der Beleuchtungsstärke \\
\hline $\mathrm{m}$ ü. NN & Meter über Normal Null \\
\hline $\mathrm{mm}$ & Millimeter als Längeneinheit \\
\hline $\mathrm{mm}$ & Millimeter als Einheit für Niederschlag, $1 \mathrm{~mm}=1$ Liter Regen auf einem Quadratmeter Boden \\
\hline MSc & Master of Science \\
\hline $\mathrm{n} . \mathrm{Br}$. & Nördliche Breite \\
\hline ö.L. & Östliche Länge \\
\hline $\mathrm{O}^{3}$ & Ozon \\
\hline $\mathrm{P}$. & Paulownia \\
\hline p.a. & Pro Jahr \\
\hline VP & Vegetationsperiode \\
\hline$\mu m$ & Mikrometer als Längeneinheit, 1 Mü = 0,001 mm \\
\hline
\end{tabular}




\section{Einleitung}

\subsection{Zum Aufbau der Arbeit}

Wie bereits am Inhaltsverzeichnis erkennbar ist, folgt dieses Manuskript keinem klassischen Aufbau. Stattdessen wurde zur besseren Darstellung der Gesamtthematik eine Unterteilung in drei Hauptkapitel vorgenommen. Diese Kapitel bauen argumentativ aufeinander auf, können aber auch separat voneinander gelesen werden.

Die Nummerierung der Überschriften folgt den Kapitelnummern, was die Orientierung erleichtern soll. Aus diesem Grund beginnt die Einleitung mit der Nummer 0.1, da sie der eigentlichen Gesamtthematik vorangestellt ist.

\section{Einleitung:}

Durch einen Prolog wird die Gattung Paulownia eingeführt und ein erster Überblick über die Gesamtthematik ermöglicht. Daran anschließend werden die Hauptfragestellungen formuliert, die als Grundlage für die Versuche dienen. Mittels einer Vertiefung dieser Fragestellungen wird ein zeitlicher Überblick über die Versuchsaufbauten gegeben und der Rahmen für die spätere Diskussion gebildet.

\section{Kapitel I:}

Das gesamte erste Kapitel widmet sich der Gattung Paulownia und erklärt deren Verbreitung, Taxonomie, Ökologie sowie abiotische und biotische Risiken. Weitere thematische Schwerpunkte werden auf die Vermehrung der Baumart und die Eigenschaften sowie die Verwendung des Holzes gelegt.

\section{Kapitel II:}

Das zweite Kapitel beginnt mit einem Überblick zur Frage der Invasivität, bei der Einbringung gebietsfremder Arten und beschreibt Kriterien zur Bewertung und Möglichkeiten im Umgang mit der Baumart.

Dem schließen sich Erläuterungen zu Kurzumtriebsplantagen und Agroforstsystemen an, um damit mögliche Anbauverfahren der Paulownia in Deutschland vorzustellen.

Schwerpunkte bilden hierbei der rechtliche Rahmen, die Etablierung und die Bewirtschaftung solcher Landnutzungssysteme sowie naturschutzfachliche Betrachtungen zum Anbau von Paulownia im Freiland.

\section{Kapitel III:}

Das gesamte dritte Kapitel widmet sich der ausführlichen Darstellung der Versuche im Freiland und im Gewächshaus, in der Reihenfolge wie sie in der Einleitung aufgeführt werden. Das Kapitel schließt mit einer ausführlichen Diskussion der in der Einleitung formulierten Fragestellungen ab und erweitert diesen Rahmen um die in Kapitel II beschriebenen Landnutzungssysteme und der damit verbundenen Problematik der Invasivität. 


\subsection{Prolog}

\subsubsection{Some time ago ...}

"Some time ago I received a foreign seed, which produced a tree. This tree I kept two years in the hot-house because I had but a single specimen, and I was fearful of losing it. But soon after finding that the shelter did not suit its habits, I planted it in the open air. There it found a temperature similar to that of its native country. It soon developed itself with great luxuriance. The leaves became at least ten times larger than when in the hot house, which was probably too warm for it. Here it soon showed its flower and fruit and was in fact the fine tree from Japan to which botanists have since given the name of Paulownia imperialis. I am far from wishing to boast of having naturalized or acclimated it, since we cannot say that its nature has changed, or that it would not have stood at first with the greatest facility in our climate. But we can say that it finds at Paris almost the same temperature as in Japan, and that it thrives very well here."

Verfasst wurde diese Beschreibung von Joseph Henri Francois Neumann, Botaniker und zuständig für die Gewächshäuser des Jardin des Plantes in Paris. Es wurde aus dem Französischen ins Englische übersetzt von Andrew Jackson Downing und veröffentlicht in „The Horticulturalist and Journal of Rural Art and Rural Taste“ von 1846 (David, 2012).

Diese Schilderung bezieht sich auf den vermutlich ersten Baum der Gattung Paulownia in Europa, welcher als Mutterbaum aller Paulownien in Frankreich damaliger Zeit gilt. Browne (1851) beschreibt in seinem Buch "Trees of America" die Besichtigung dieser Paulownia im Pariser Garten Jardin des Plantes. Der Baum wird zu der Zeit mit einem Durchmesser von zwei $\mathrm{Fuß}^{2}$ bei einer Höhe von $20 \mathrm{Fuß}$ angegeben und in der Beschreibung erschien es erwähnenswert, dass er den harten Winter von 1838 zu 1839 ohne jede Abdeckung überstanden hatte.

Die Baumart Paulownia hat durch ihre seit mehr als 200 Jahren fortschreitende weltweite Verbreitung nicht nur viele Kosenamen wie bspw. Empress (Kaiserin) oder Princess (Prinzessin) erhalten (Snow, 2015).

Der Baum ist auch in vielen alltäglichen Formen präsent. Beispielsweise findet sich in Japan auf der 500-Yen-Münze die Abbildung eines P.-Blütenstandes. Weiterhin schmückt die „5-7-5 Paulownie“, wie sie inoffiziell genannt wird, das ursprüngliche Wappen der Familie der Ashikaga-Fürsten, was heutzutage dem Wappen des japanischen Premierministers bzw. der Regierung entspricht.

Auch in dem Buch „Romanov Prophecy“, einem New York Times Best-Bestseller aus dem Jahr 2005, spielt die Baumart eine Hauptrolle (Berry, 2005).

${ }^{2} \mathrm{Fuß}=$ verschiedenartig verwendetes Längenmaß, $1 \mathrm{Fuß}$ entspricht zwischen 28 bis $32 \mathrm{~cm}$. 
Selbst in dem Film „Rocky“ von 1976 hat sie einen kleinen Cameoauftritt, wenn die Straßen von Philadelphia (Benjamin Franklin Parkway) gezeigt werden und der Protagonist energiegeladen zu dem Song "Gonna Fly Now“, von Bill Conti, die letzten Stufen zum „Philadelphia Museum of Art“ emporspringt, sind Paulownia-Bäume im Bildhintergrund für den geschulten Beobachter zweifelsohne erkennbar (siehe hierzu auch David, 2012; Lacy, 2000).

Die Paulownia scheint im Alltag fast allgegenwärtig und bleibt doch so häufig unbemerkt. Das hier vorliegende Manuskript soll einen vertiefenden Einblick ermöglichen und für die zukünftige Wahrnehmung von Paulownia-Bäumen in der unmittelbaren Umgebung sensibilisieren.

\subsubsection{Einführung in die Thematik}

Der Mensch besitzt die Fähigkeit die inn umgebende Umwelt zu formen. Ob dieser Gestaltungswille dabei aktiv und zielgerichtet erfolgt oder passiv eine Form von Unachtsamkeit darstellt, ist für die dabei ausgelösten bzw. sich verändernden, natürlichen Prozesse irrelevant.

Schon vor dem Übergang von einer Jäger- und Sammlerkultur hin zu einer Hirten- und Bauernkultur - eine Zeit, die wir rückblickend als Jungsteinzeit bezeichnen (Neolithikum, ca. 9.500 - 1.900 v.Chr.) - wurden Pflanzenarten, durch die Wanderbewegungen des Menschen, in neue Lebensräume überführt. Die sich anschließende Domestizierung, von für den Menschen nützlichen Pflanzen, hält bis in die heutige Zeit an.

Die Gattung Paulownia ist eine der am längsten vom Menschen aktiv angebauten Baumarten der Welt, deren erste schriftliche Erwähnung bis noch vor 1000 v.Chr. zurückgeht (Barton, 2007). Sie gehört zu den schnellwachsenden Baumarten und ihre Samen können an den unwirtlichsten Orten gedeihen (Remaley, 2005; Snow, 2015).

Seit vielen Jahren schon wird sie in Baumärkten angeboten und wächst daher vielfach als Ziergehölz in deutschen Gärten und das obwohl ihr natürliches Verbreitungspotential (siehe Invasivität, Kapitel II) unter mitteleuropäischen Klimabedingungen noch immer nicht abschließend geklärt ist.

Sie benötigt, gerade in der Jugendphase, viel Licht und kann nur bei ausreichender Wasserversorgung, innerhalb weniger Jahre, große Mengen an Biomasse erzeugen. Daher verwundert es nicht, dass die Baumart auch wirtschaftlich in Form von Kurzumtriebsplantagen genutzt wird (Cathaia, 2020).

Zur erfolgreichen Etablierung einer neuen Art, außerhalb ihres natürlichen Verbreitungsgebietes, bedarf es jedoch des Verständnisses einer ganzen Reihe von pflanzlichen Bedürfnissen. Abiotische ${ }^{3}$ und biotische ${ }^{4}$ Umweltfaktoren nehmen darauf mannigfaltigen Einfluss. Sie können in Maßen bspw. durch die Wahl des Standortes und der Bewirtschaftung beeinflusst werden.

Die biotischen Umweltfaktoren sind nur randläufig Gegenstand der weiteren Betrachtungen (siehe Kapitel I).

\footnotetext{
${ }^{3}$ abiotisch: (griech. $=$ nichtlebend), bezeichnet alle Umweltfaktoren an denen Lebewesen nicht beteiligt sind, wie z.B. Temperatur, Licht, Klima, usw.

${ }^{4}$ biotisch: alle Umweltfaktoren an denen Lebewesen beteiligt sind.
} 
Bei den abiotischen Umweltfaktoren hingegen ist das vorherrschende Klima im Anbaugebiet von größerer Bedeutung. Die ökologischen Parameter wie Licht und Wasser beeinflussen das Wachstum der Pflanzen direkt. Dabei entscheiden die ersten Vegetationsperioden sowohl über den Anwuchserfolg als auch über die weitere Entwicklung der Pflanzen. Daher wird diese zeitliche Periode im Folgenden näher wissenschaftlich besprochen.

Als Untersuchungsobjekte wurden die drei weltweit am häufigsten angebauten P.-Arten ausgewählt:

Am bekanntesten, weil in der Fachliteratur am ausführlichsten beschrieben, ist die Art P.tomentosa. Sie wird aufgrund ihres geraden Wuchses und vor allem wegen ihrer Frosthärte für den Anbau in Mitteleuropa bevorzugt.

Die P.elongata zeigt das schnellste Wachstum der Gattung und wird daher häufig für Züchtungen bzw. Hybridisierungen verwendet. Sie ist allerdings nur in Maßen winterhart. Die P.fortunei lässt sich bezüglich ihrer Wachstumsgeschwindigkeit und ihrer Toleranz gegenüber Minustemperaturen grob zwischen der P.tomentosa und der P.elongata einordnen. Aufgrund ihres guten Wuchsverhaltens, bei dem meist keine Ästung nötig ist, wird sie ebenfalls weltweit angebaut.

\subsection{Ziele der Arbeit}

Das erste und zweite Kapitel widmet sich der Beschreibung der Gattung Paulownia, ihrer Verwendung und sowohl den Problemen als auch den Möglichkeiten ihres Anbaus. Zur Abrundung des Gesamtbildes werden im dritten Kapitel zwei sich ergänzende Versuchsanordnungen beschrieben. Die Fragestellungen dieser Versuche werden hier im Folgenden näher erläutert:

\subsubsection{Hauptfragestellungen}

Die drei P.-Arten wurden aufgrund ihrer Wuchseigenschaften und der Häufigkeit ihres weltweiten Anbaus als Untersuchungsobjekte ausgewählt. Ihr Wuchsverhalten in den ersten Vegetationsperioden sowohl im Freiland wie auch im Gewächshaus war Inhalt der hier vorliegenden wissenschaftlichen Beobachtungen. Die folgenden Fragestellungen begleiteten diese Untersuchungen:

Bezüglich des Anbaus im Freiland:

- Welche Wachstumsreaktionen zeigen die drei P.-Arten im Vergleich, bei einem Freilandanbau unter mitteleuropäischen Klimabedingungen?

- Zeigen sich Unterschiede beim Wachstumsverlauf im Vergleich zum natürlichen Verbreitungsgebiet der Arten?

Bezüglich der Versuche im Gewächshaus:

- Wie verändert sich das Wachstumsverhalten unter verschiedenen Licht- und Wasserverhältnissen?

- Zeigen sich dabei Unterschiede in der Entwicklung bei einem Vergleich der drei P.-Arten? 


\subsubsection{Vertiefung der Fragestellungen}

Die folgenden Untersuchungen bzw. Experimente wurden zur Vertiefung dieser Fragestellungen durchgeführt und mit einer Reihe von BSc.- und MSc.-Abschlussarbeiten unterstützend begleitet:

\subsubsection{Voruntersuchungen}

Zur Eingrenzung des Themenfeldes und zur weiteren Ausarbeitung der Fragestellungen wurden zu Beginn, neben dem üblichen Literaturstudium, auch zeitgleich erste Voruntersuchungen durchgeführt.

Im Jahr 2014 wurde ein privater Landwirtschaftsbetrieb in Brandenburg besucht und deren Paulownia-Anbau aus P.superelongata (eine Kreuzung aus P.elongata und P.fortunel) auf 2,7 ha mittels einer BSc.-Arbeit ausgewertet:

- Anbaumöglichkeiten und Risiken von Paulownia spec.“ (Nobis, 2014)

In der darauffolgenden Vegetationsperiode fanden erste Gewächshausversuche in Göttingen zum Wachstumsverhalten und zur Trockenresistenz der Arten P.elongata, P.fortunei und P.tomentosa statt. Eine Auswertung erfolgte in Form einer BSc.-Arbeit:

- „Jugendwachstum und Trockenresistenz von verschiedenen Paulownia-Arten“ (Büker, 2015).

\subsubsection{Freilandversuche}

Auf Grundlage dieser Voruntersuchungen wurden im Frühjahr 2015 in Brandenburg, in Göttingen und auf der Nordseeinsel Föhr mehrjährige Freilandversuche mit den Arten P.elongata, P.fortunei und P.tomentosa angelegt. Es sollte das Wuchsverhalten unter den klimatischen und standörtlichen Bedingungen und unter dem Einfluss verschiedener Bodensubstrate wie bspw. Geohumus und mittels künstlicher Mykorrhizierung beobachtet werden. Die folgenden Annahmen wurden dabei näher betrachtet:

Die klimatischen und standörtlichen Bedingungen beeinflussen direkt

- sowohl das Anwuchsverhalten als auch

- das Längenwachstum der P.-Pflanzen.

Bezüglich der verschiedenen Behandlungsarten:

- Der Einsatz von Geohumus führt zu einer Verbesserung des Längenwachstums der Pflanzen und verringert die Ausfallrate.

- Eine Mykorrhizierung des Pflanzloches erhöht das Längenwachstum der Pflanzen.

Die P.-Arten zeigen:

- Arttypische Unterschiede (Längenwachstum) in der Reaktion auf die klimatischen und standörtlichen Bedingungen.

- Diese arttypischen Reaktionen werden sich von Vegetationsperiode zu Vegetationsperiode weiter verstärken. 


\subsubsection{Gewächshausversuche}

In Ergänzung zu den Freilandversuchen wurde im Frühjahr 2016 ein für zwei Vegetationsperioden geplanter Gewächsversuch in Göttingen angelegt. Hierbei sollte das Wuchsverhalten von den Arten P.elongata, P.fortunei und P.tomentosa unter verschiedenen Beschattungs- und Bewässerungsstufen beobachtet werden.

Die folgenden Annahmen sollten dabei näher untersucht werden:

Es ist davon auszugehen, dass durch die Verringerung des Lichts:

- Das Längenwachstum der Pflanzen zunimmt,

- das Durchmesserwachstum im Verhältnis dazu abnimmt und,

- weniger Biomasse gebildet wird.

Eine Verringerung der Wasserzufuhr wird:

- Das Längenwachstum negativ beeinflussen und

- sowohl das Durchmesserwachstum,

- als auch die Bildung von Biomasse verringern.

Die P.-Arten werden:

- Unterschiedlich starke Reaktionen auf die verschiedenen Beschattungs- und Bewässerungsstufen zeigen.

- Im Vergleich von der ersten zur zweiten Vegetationsperiode werden sich die für die Art typischen Reaktionen der Pflanzen verstärken.

Im fachlichen Zusammenspiel mit den verschiedenen Gewächshausversuchen entstanden die folgenden Abschlussarbeiten:

BSc.-Arbeiten:

- „Reaktionen von zweijährigen Paulownia-Stockausschlägen auf unterschiedliche Wasserversorgung" (Hoffmann, 2017).

- „Biomasseentwicklung von Paulownia elongata, fortunei und tomentosa bei unterschiedlichen Bewässerungsstufen“" (Wortmeier, 2017).

- „Reaktionen von zweijährigen Stockausschlägen der Arten Paulownia elongata, fortunei und tomentosa auf unterschiedliche Beschattung“ (Eckardt, 2018).

MSc.-Arbeiten:

- „Jugendwachstum von Paulownia elongata, fortunei und tomentosa bei unterschiedlicher Lichtintensität" (Dannenberg, 2016).

- „Biomassenzuwachs von Jungpflanzen der Arten Paulownia elongata, Paulownia fortunei und Paulownia tomentosa unter modifizierten Standortbedingungen“ (Hoffmann, 2019).

\subsubsection{Weiterführende Überlegungen}

In weiterführenden Betrachtungen ergeben sich Fragen nach der Anbauwürdigkeit und Anbaumöglichkeit der Baumarten. Die in Deutschland zurzeit dafür denkbaren Landnutzungsysteme sind Kurzumtriebsplantagen und Agroforstsysteme (bspw. auf Dauergrünland). Im Zusammenhang mit diesen Überlegungen wurden die folgenden Abschlussarbeiten begleitet und thematisch miteinbezogen: 
BSc.-Arbeiten:

- „Die Bedeutung der Niederwaldbewirtschaftung in ausgewählten Ländern Europas“ (Meyerhof, 2014).

- „Umtriebszeiten von Kurzumtriebsplantagen“ (Hofheinz, 2016).

- „Niederwaldumwandlung / - überführung am Beispiel der SIEGERLÄNDER Hauberge“" (Setzer, 2019)

MSc.-Arbeit:

- "Paulownia tomentosa - der Blauglockenbaum in der landwirtschaftlichen Nebennutzung auf Dauergrünland - Eine Beispielrechnung" (Thielen, 2019).

Weitere Betrachtungen zur Anbauwürdigkeit und Anbaumöglichkeit der Baumart finden sich im Kapitel II und Kapitel III dieses Manuskripts.

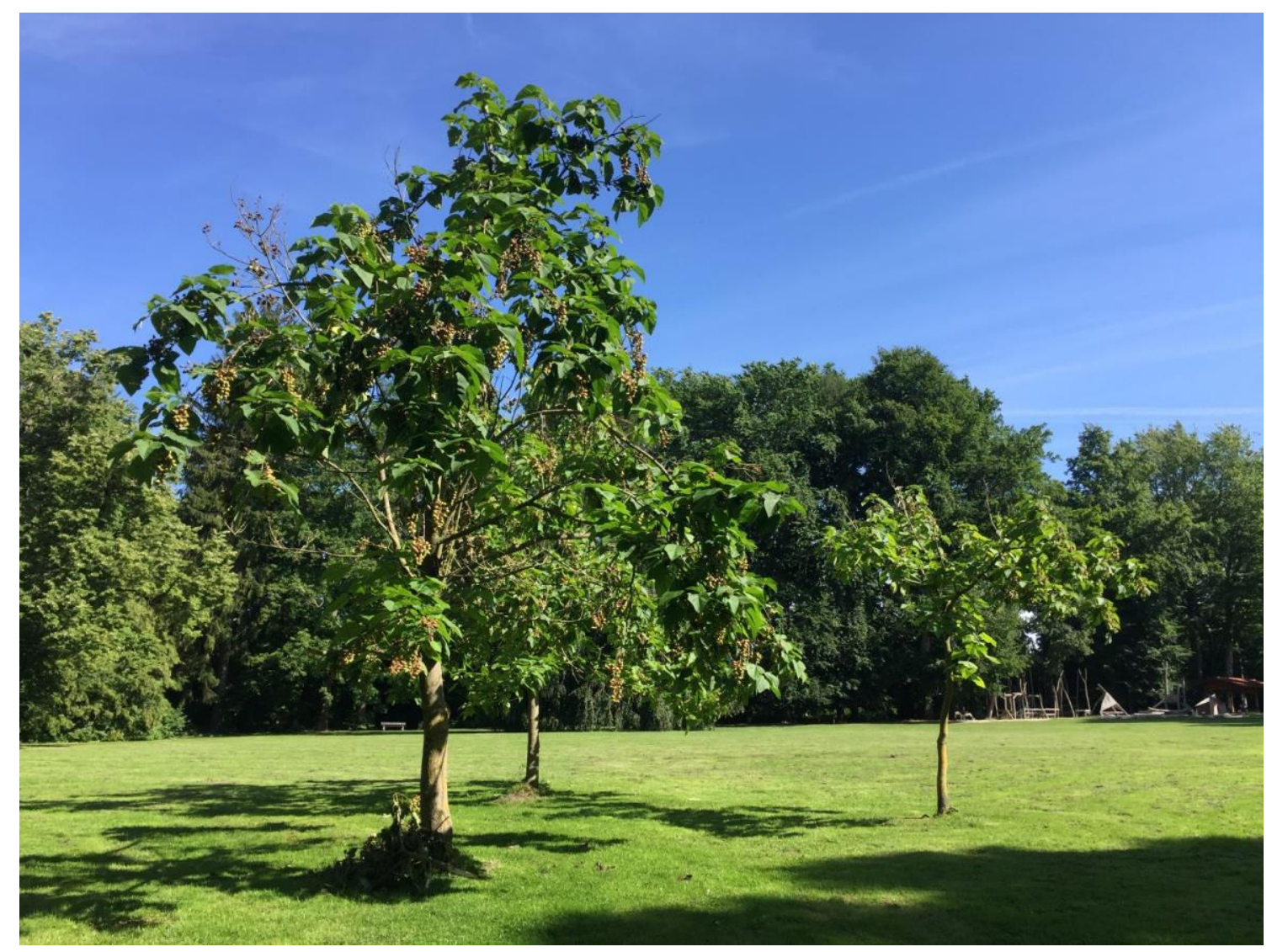

Abb. 2: Paulownia-Bäume auf den südlichen Schillerwiesen in Göttingen. 


\section{Kapitel I}

\section{Paulownia}

\subsection{Die Gattung Paulownia}

Die Gattung Paulownia und mit ihr die weltweit bekannteste, weil am häufigsten angebaute Vertreterin ihrer Art, die P.tomentosa ist einer der wichtigsten Baumarten Chinas mit über einer Milliarde Plantagen-Bäumen, die jährlich ca. 3 Mio. Kubikmeter Holz erzeugen, was einem Wertzuwachs von 55 Mio. US-Dollar entspricht (Cabi, 2019). Ihre Fähigkeit des schnellen Holzzuwachses hat über der ökonomischen Bedeutung hinweg im Laufe der Geschichte auch tiefe soziale Auswirkungen auf die chinesische Bevölkerung und ihren Traditionen gehabt. So wird die Baumart damals wie heute vielfältig in der chinesischen Medizin verwendet und hat so auch ihren Weg in zahlreiche mystische Geschichten gefunden.

Eine Bewertung ihres Einflusses auf ihre Umwelt und speziell auf die von ihr besiedelten Waldgesellschaften, fällt weltweit sehr verschieden aus und befindet sich auch weiterhin im Wandel. Die Bewertungen sind dabei stark von den vorherrschenden Naturschutzbestimmungen (Invasivitäts-Frage) der jeweiligen Länger geprägt und hängen im direkten Zusammenhang mit dem aktiven Anbau bspw. in Form von Plantagen oder mit der indirekt einhergehenden natürlichen Verbreitung der Art und deren Folgen ab.

\subsubsection{Herkunft und Verbreitung}

\subsubsection{Namensherkunft}

\subsubsection{Historische Erwähnungen}

Der Baum ist tief in der asiatischen Mythologie verankert - vermutlich wegen seines Erscheinungsbildes, seiner Widerstandskraft und seiner Fähigkeit sehr viele Samen zu produzieren, die sich schnell, sehr weit verbreiten können (bis zu 9,7 km laut Remaley (2005); siehe auch Snow (2015)). So verwundert es also nicht, dass sowohl in China als auch in Japan der Baum mit Stärke, Reinheit und Erneuerung in Verbindung gebracht wird (Lacy, 2000).

Die von dem Schreiber Chen Chu (1061 - 982 v.Chr.) verfasste „Monographie der Paulownia“ ist der erste schriftliche Nachweis über die Baumart im asiatischen Raum (Barton, 2007). In ihr werden die Kultivierung und die Nutzung des Holzes beschrieben und die vielen erwähnten Details im Bereich des Anbaus der Jungpflanzen, der Wiederaufforstung und des Waldmanagements sind selbst nach so langer Zeit für die heutige Forschung noch von großem Wert (Zhu et al., 1986).

Beispielsweise gibt es eine alte Sage nach der der chinesische König Yui (ca. 600 v.Chr.) mit einem Sarg zu Grabe getragen wurde, welcher aus 3 „cuen“ (alte chin. Maßeinheit, 1 cuen $=3 \mathrm{~cm}$ ) dickem Paulownia-Holz bestand. 
Bemerkenswert ist auch eine frühe Erwähnung des Holzes in der „Erya“, einer vorzeitlichen chinesischen Enzyklopädie, die vermutlich mit ihrem Hauptwerk zwischen dem vierten und zweiten Jahrhundert v.Chr. entstand (Barton 2007).

Im „Zhuangzi - Das wahre Buch vom südlichen Blütenland“, welches um 300 v.Chr. von dem chinesischen Philosophen und Dichter Zhuang Zhou verfasst wurde und als eines der Hauptwerke des Daoismus gilt, wird beschrieben wie der Vogel Phoenix, welcher in einem Meer aus Flammen stirbt und aus seiner eigenen Asche aufersteht, vom Südzum Nordmeer fliegt und dabei nur auf den heiligen Bäumen (Paulownia) rastet.

Im alten Japan gibt es diesbezüglich eine ähnliche Geschichte nachdem der Ho-o, eine Kreatur die dem Phoenix ähnelt, ebenfalls sein Zuhause in diesem Baum findet (zitiert nach Meyer, 2012). Diese Mythen haben vermutlich den realen Hintergrund, dass der Baum, nachdem er gefällt wurde, schnell wieder austreiben - also wortwörtlich wiederauferstehen kann.

Die erste Anlage einer Plantage wird auf das Jahr 221 v.Chr. zu Zeiten der ersten Dynastie des chinesischen Kaiserreiches der Qin (221 - 207 v.Chr.) datiert (Barton, 2007). Dies geht einher mit einer Erwähnung in dem „Buch der Dokumente - Shang Shu“, welches um 300 v.Chr. verfasst wurde und beschreibt wie die Bauern der Provinz ihren König regelmäßigen mit Paulowniaholz belieferten.

In der „Zeit der Streitenden Reiche“ („Zhànguó Shídài“ 475 - 211 v.Chr.) entstand das Buch "Mencius" in welcher die ersten Formen zur Kultivierung von Bäumen in China beschrieben werden. Es findet sich darin die Regel, dass nur der ein guter Bauer sei, der immer auch eine Paulownia und einen Catalpa ovata (Gelber Trompetenbaum) pflanzt. (Zhu et al., 1986)

Der Baumart werden sowohl in China als auch in Japan große Heilkräfte nachgesagt. Beispielsweise soll eine Mixtur aus den Blättern gegen Fieber, Warzen und auch gegen Kahlköpfigkeit helfen, und die Blüten bei Problemen mit der Leber Milderung versprechen. (Foster and Duke, 2000)

Noch heute gibt es in Japan die Tradition, dass bei der Geburt einer Tochter mehrere Paulownia gepflanzt werden. Wenn die Tochter dann alt genug ist, um in die Ehe zu gehen, so sind die Bäume ausgewachsen und ihr Holz wird als Mitgift verwendet (Zhu et al., 1986).

Aber auch über den asiatischen Kontinent hinaus gibt es historische Erwähnungen in neuerer Zeit. Beispielsweise wurden in einer Vielzahl von amerikanischen Bundesstaaten P.-Fossilien gefunden, die erdgeschichtlich dem Zeitalter des Tertiärs (vor 2,6 - 66 Mio. Jahren) zugeordnet werden können. Es wird vermutet, dass die Gattung ursprünglich über die gesamte nördliche Hemisphäre (heutiges Alaska, Nordamerika und Europa) verbreitet war und erst durch verschiedene aufeinanderfolgende Eiszeiten ausgestorben ist. (Smiley, 1961)

\subsection{Entstehung des westlichen Namens}

Erst durch die Einführung der Baumart in die westliche Hemisphäre kam es zu dem heutigen allseits bekannten königlichen Beinamen: Empress oder Princess (Snow, 2015).

Karl Thunberg, ein schwedischer Botaniker, hat die Baumart nach seinem Besuch Japans im Jahre 1770 erstmalig in der europäischen Literatur erwähnt (Lacy, 2000). 
Der Name Paulownia leitet sich ursprünglich von der niederländischen Aussprache der russischen Prinzessin Anna Pavlovna (1795-1865), Enkeltochter der Kaiserin Katharina die Große (1729-1796) und Tochter des Zaren Paul I (1754-1801) ab. Im Jahre 1816 heiratete sie den niederländischen König William II (1792-1849) und war von seither als Anna Paulowna bekannt.

Anfang des 19. Jahrhunderts wurde in den damaligen Niederlanden an der Nordwestküste ein Polder trockengelegt und nach ihr benannt, auf dem sich heutzutage das größte zusammenhängende Blumenzuchtfeld der Welt erstreckt.

Ursprünglich wurde die Gattung Paulownia der Familie Scrophulariaceae (Braunwurzgewächse) zugeordnet, was auch heute noch gerne geschieht (Cabi, 2019). Allerdings führten neuere molekularbiologische Untersuchungsmethoden zur Bildung einer eigenständigen Familie der Blauglockenbaumgewächse (Paulowniaceae) (APG III, 2009). Die erstmalige Erwähnung der Familie der Paulowniaceae erfolgte 1949 durch den japanischen Botaniker Takenoshin Nakai (1882-1952) in einem Artikel im „Journal of Japanes Botany“ (Nakai, 1949).

\subsubsection{Natürliche Verbreitung}

Auf Grund der nachweislichen Jahrtausende langen Nutzung der Baumart in China und der damit einhergehenden anthropogenen Streuung der verschiedenen Arten ist eine genaue Bestimmung der natürlichen Verbreitung nicht mehr möglich. Jedoch bietet die ökologische Amplitude eine gute Grundlage zur Beschreibung der Extrema in welchen die Baumart gedeiht und lässt somit Rückschlüsse auf die natürliche Verbreitung zu.

Die Gattung Paulownia erstreckt sich von ihrem natürlichen Verbreitungsgebiet von Indochina über Taiwan und China bis nach Korea und Japan (Abb. 3).

Die von Zhu et al. (1986) beschriebenen neun Arten sind alle auf das chinesische Festland begrenzt, mit Ausnahme der P.fortunei die ebenfalls auch in Laos und Vietnam wächst und P.tomentosa die auch über Japan und Korea hinweg zu finden ist. Im Norden ist die Baumart begrenzt auf die Gebiete südlich von Liaoning, Beijing, Taiyuan, Yianan und Pinglian. Im Osten erstreckt sich ihre Verbreitung von Gansu über Szechuan bis nach Taiwan und im Süden von Kwangtung bis Kwangsi und im Südwesten bis in die Gebiete von Yunnan. Sie erreicht dabei Höhen von $1.900 \mathrm{~m}$ im westlichen Honan bis zu $2.400 \mathrm{~m}$ im Süden Chinas und den Bergregionen im Südwesten. (Zhu et al., 1986)

Im mittleren Verlauf des Yangtse-Flusses, von der westlichen Hupeh-Provinz bis zur Region der Drei-Schluchten im östlichen Szechuan, überlappt sich das Verbreitungsgebiet der meisten Arten, weswegen Zhu et al. (1986) davon ausgehen, dass sich hier eines der Ursprungsgebiete der Art befindet. Nur P.catalpifolia und P.taiwaniana sind die einzigen beiden Arten die dort nicht vorhanden sind.

In diesem vermuteten Ursprungsgebiet kommt es zur Ausbildung einer Vielzahl von natürlichen Hybriden, was deren genaue Bestimmung deutlich erschwert. Es ist aber zu vermuten, dass dies nicht das einzige Zentrum zur Ausbreitung der Art ist, da die meisten Baumarten im Laufe ihrer natürlichen Ausdehnung sich den unterschiedlichen Umweltbedingungen anpassen und somit eine Vielzahl von Variationen innerhalb einer Art bilden können. Als Beispiel gilt hier die P.tomentosa in der Sheng Long Jia Bergregion (Hupeh-Provinz) die dort ein großes Spektrum an Variationen ausgebildet hat. (Zhu et al., 1986) 
Auch Funde von Paulownia-Fossilien in amerikanischen Bundesstaaten legen nahe, dass die Art vor den letzten Eiszeiten um ein vielfaches weiter verbreitet war, als es
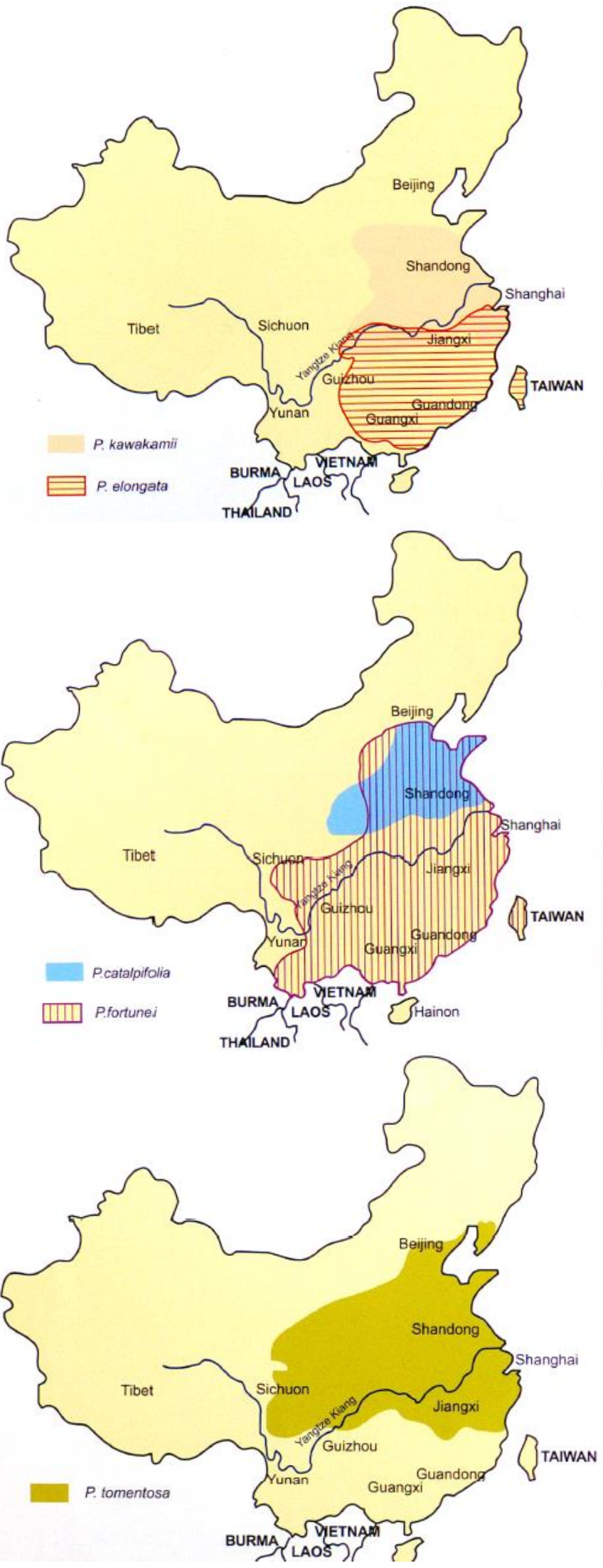

Abb. 3: Natürliche Verbreitungsgebiete der PaulowniaArten: P.elongata, P.fortunei, P.tomentosa; verändert nach Barton (2007). heute der Fall zu sein scheint (Smiley, 1961). Zhu et al. (1986) weisen in einer früheren Veröffentlichung von 1980 daraufhin, dass Paulownia auch Charakteristika von subtropischen und tropischen Arten besitzt und erklären so die Verbreitung aller Arten auch südlich des YangtseFlusses bei südtropischen Klima mit Ausnahme von P.australis, P.fortunei und P.kawakamii die darüber hinaus auch noch im tropischen Klima gedeihen können.

\subsubsection{Der Weg nach und durch Europa}

Nach Europa und zuallererst nach Frankreich gelangte die Paulownia um 1830 durch Philipp Franz von Siebold (1796-1866), wobei sie namentliche Erwähnung in der „Flora Japonica, Sectio Prima (Textband I) (1835)" fand.

Von Siebold war ein bayerischer Arzt und leidenschaftlicher Ethnologe und Botaniker und zu Beginn des 19. Jahrhunderts im Dienste der Niederländischen Ostindien-Kompanie (niederländisch: VOC, Vereenigde Oostindische Compagnie) in, dem zu der Zeit noch stark von ausländischen Einflüssen abgeschotteten, Japan beschäftigt. Auf Grundlage seines medizinischen Wissens und seines Interesses für die japanische Fauna und deren Verwendung in der dortigen Heilmedizin, sammelte er eine Vielzahl von Pflanzen die er nach Europa verschiffte. (David, 2012) 
Bereits seit Jahrzehnten ist Paulownia nun schon in ganz Europa verbreitet, wobei sie aber bisher nur selten in für den sie natürlichen Vegetationstypen wie bspw. Auenstandorte und Waldlichtungen anzutreffen ist (Essel, 2007) und das obwohl sie bspw. in Italien seit 1989 in Form von Plantagen und als Windschutz angebaut wird (Mezzalira and Colonna, 2002). Die verwilderten, synanthropen ${ }^{5}$ Vorkommen in Italien wurden klassifiziert und gelten als etabliert (Wilhalm et al., 2002).

In der Schweiz wird die Art für Basel ebenfalls als etabliert eingestuft, da sie an vielen Orten in der Stadt als verwilderte Einzelexemplare anzutreffen ist, die sich aber nicht zu großen Bäumen weiterentwickeln (Brodtbeck et al., 1999). Für Zürich wird von ersten Ausbreitungen seit 1985 berichtet und die Art gilt seitdem als sich schnell verbreitend (Landolt, 1993).

Aus weiteren europäische Länder wird von Sichtungen berichtet, die oft im Zusammenhang mit bereits vorhandenen Plantagebäumen stehen (Essel, 2007).

Im deutschsprachigen Raum wurde das erste verwilderte Vorkommen (P.tomentosa) 1925 beschrieben. In den wärmeren Regionen von Baden-Württemberg und Rheinland-Pfalz ist seit den 1970er und 80er Jahren von weiteren verwilderten Vorkommen berichtet worden (Kiermeier, 1977). In den 1990er Jahren wurden nur noch wenige weitere Sichtungen verwilderter Einzelexemplare gemeldet (Adolphi, 1997; Keil and Loos, 2004).

Die Art gilt laut Buttler \& Thieme (2013) heutzutage in den Bundesländern Baden-Württemberg, Hessen, Nordrhein-Westfalen als eingebürgert. Weitere noch unbeständige Vorkommen wurden in Berlin, Bayern, Niedersachsen und Rheinland-Pfalz gesichtet. Dabei handelt es sich zumeist um Grenzstandorte in urbanen Bereichen wie bspw. Bahndämme, Mauerritzen, usw. (Keil and Loos, 2004) (Abb. 4).

Vorteilig für die Art ist ihre Trockenresistenz und dass sie selbst hohe Temperaturen übersteht, wie sie zumeist bei exponierter Lage im Sommer auftreten können. Hecker \& Weisgerber (2014) sprechen in diesem Zusammenhang von einem für die Pflanze sogar förderlichen „Kachelofeneffekt“.

Eine der häufigsten Formen der Verbreitung erfolgt durch Kurzumtriebsplantagen auf deutschen Ackerflächen und hierbei bevorzugt vor allem P.tomentosa und P.elongata (Cathaia, 2020). Aber auch andere P.-Arten und zahlreiche Hybride werden vermehrt im Pflanzenhandel angeboten

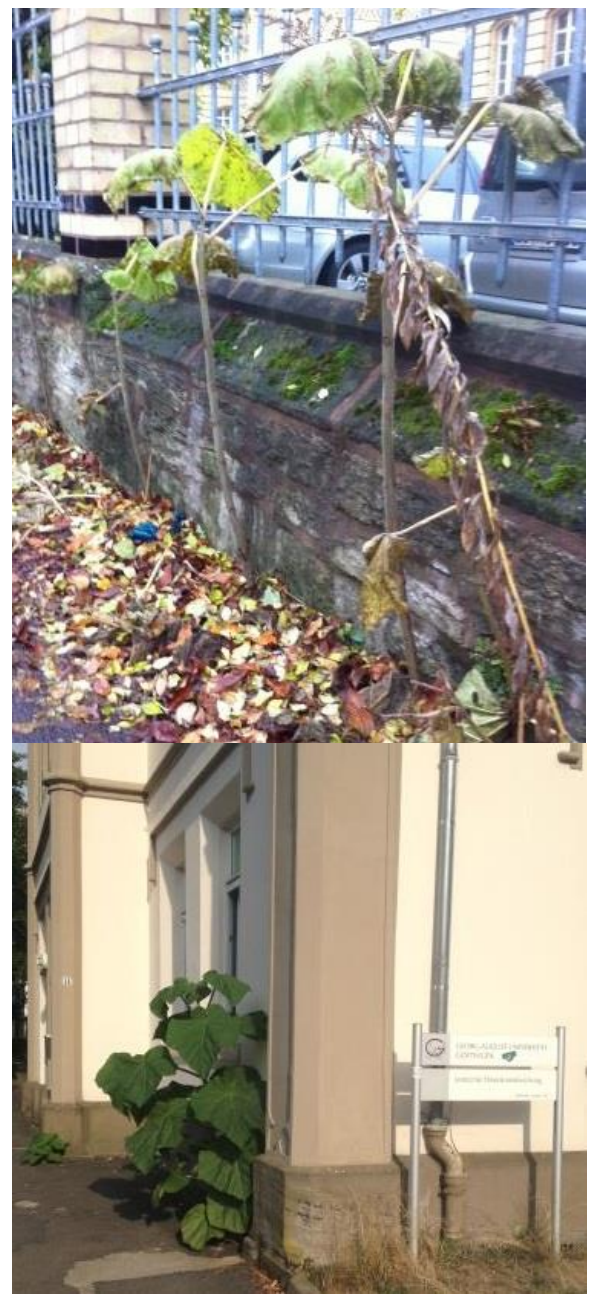

Abb. 4: Oberes Bild zeigt einjährigen Paulownia-Wildwuchs in der GoßlerStr.; Unteres Bild zeigt einjährigen Paulownia-Wildwuchs am Institut für Demokratieforschung, in der WeenderLandstr., in Göttingen. (Stimm et al., 2013) und finden sich in deutschen Gärten wieder.

${ }^{5}$ Synanthropie $w$ [von griech. synanthrōpein = mit den Menschen leben; Adj. synanthrop], mehr oder weniger fest an den engeren Siedlungsbereich des Menschen gebundenes Auftreten von Organismen (hemerophil, Kulturfolger). Quelle: LexBio (2020) 


\subsubsection{Verbreitung in den USA}

1.1.1.4.1 Wiedereinführung auf dem amerikanischen Kontinent Das Wiedereintreffen der Baumart auf dem amerikanischen Kontinent, nachdem die Art vermutlich schon im Tertiär vorhanden war (Smiley, 1961), gibt Shiu-Ying (1961) mit der Jahreszahl 1844 an, wobei sich durch die Zeitschrift "The American Agriculturalist“" von August 1843 bereits eine erste Erwähnung, mit dem Verweis auf die „Parson's Nursery“ in Queens, belegen lässt.

Allerdings wird davon ausgegangen, dass auch schon weit früher P.-Samen in die USA gelangten, da die Samenkapseln (Abb. 5) vielfach als Verpackungsmaterial für den Warentransport aus China verwendet wurden. (David, 2012)

Diese frühe, unkontrollierte Form der Einführung einer fremden Art in die USA stellt, neben den von Staats wegen her immer wieder neuaufgelegten Programmen zu Wiederaufforstungszwecken bzw. zur Verhinderung von fortschreitender Bodenerosion oder einfach nur aus Gründen von Stadtverschönerungen (siehe hierzu auch David, 2012; Lacy, 2000), keine Ausnahme dar sondern entspricht eher der Regel, wie Snow (2015) beiläufig bemerkt.

Die amerikanische Holzindustrie verwendet Paulownia und dabei bevorzugt P.elongata seit 1970 (Snow, 2015), wobei die Meinungen über die Baumart von damals bis heute großen Schwankungen unterliegen. Hauptauslöser für diese Entwicklung war der japanische Holzmarkt, der zu der Zeit seinen Holzverbrauch nicht mit der heimischen Produktion decken konnte, was wiederum den Preis für P.-Holz stark nach oben trieb (Kays, 1996).

Hinzu kam bspw. in Virginia noch der Hurrikan Camille dessen großräumige Windwürfe von den Waldbesitzern mit P.tomentosa aufgeforstet wurden (Williams, 1993).

Landbesitzer wurden in den USA in damaliger Zeit aktiv durch Werbeprospekte von der Überlegenheit dieser „magic-tree“ Baumart informiert um den Anbau zu fördern. Schnell wurden aber Gegenstimmen laut, die nicht daran glauben wollten, dass ein Wachstum auf jedem Standort möglich wäre, es keine Baumkrankheiten bei dieser neuen Art gebe und selbst Baumpflegemaßnahmen nicht nötig wären.

Trotz dessen entwickelte sich die Art in den 70er Jahren zur neuen Trendbaumart und bereits 10 Jahre später war nicht mehr so klar, wie Snow (2015) erwähnt, ob sich die getätigten Investitionen lohnen würden bzw. ob der Trend nicht doch ein jähes Ende finden könnte.

Aber auch andere Länder wie China, Australien und Brasilien begannen aktiv um 1970 herum die Baumart für den Handel mit Japan in Form von Plantagen zu kultivieren (DMN,

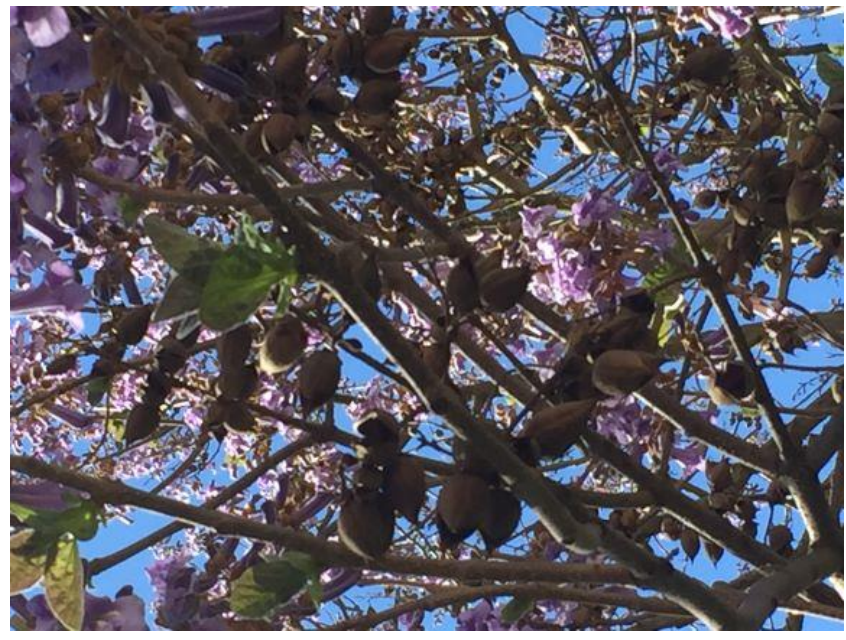

Abb. 5: Blütenstände und Samenkapseln einer Paulownia, in der Goßler-Str., in Göttingen. 1975). 


\subsection{Anbau- und Invasivitätsproblematik in den USA}

Im Mai 1983 kam es in Philadelphia (Pennsylvania) zu einem Holzdiebstahl, der von den Medien als „Fairmount Park Chainsaw Massacre" betitelt wurde, was den Hype um die Baumart ein weiteres Mal entfachte. Der Holzpreis auf dem japanischen Markt hatte zu der Zeit die 1.000 Dollar Marke, für ca. drei Meter lange Stammenden, überschritten und so plünderten zahlreiche Holzdiebe die Parks von Delaware, New York City, New York und Wilmington auf der Suche nach Paulownia-Bäumen. (Lacy, 2000)

So verwundert es nicht, dass zu der Zeit erneut zahlreiche Plantagen angelegt wurden, wobei P.elongata am häufigsten Verwendung fand und es zu Züchtungsversuchen von Hybriden kam, mit dem Ziel ein noch schnelleres Wachstum und höhere Robustheit bei Kälte zu ermöglichen (Hall, 2008). Im Zuge dessen wurde 1991 die „American Paulownia Association" (APA) gegründet, um Plantagenbesitzer miteinander zu vernetzen und Anbauerfahrungen auszutauschen. $\mathrm{Zu}$ der Zeit beruhte der gesamte japanische Holzimport zu dreiviertel auf Paulowniaholz. (APA, 1991)

Snow (2015) beschreibt erste Probleme mit der invasiven Verbreitung von speziell P.tomentosa in den 1940er Jahren in den USA, was eine Verschiebung des Anbaus mehr hin zu P.elongata zur Folge hatte.

Die Executive Order 13112 (1999), die vom damaligen Präsidenten Bill Clinton unterzeichnet wurde, führte zur Gründung des „National Invasive Species Council“ der darüber entscheiden sollte, ob Paulownia eine nichtheimische invasive Art ist. Die Verbreitung von P.tomentosa erstreckte sich zu dem Zeitpunkt auf über 27 Staaten von Main bis Texas (Beasley and Pijut, 2010).

Die Bundesstaaten Connecticut, Georgia, Kentucky, Louisiana, Maryland, North Carolina, New Jersey, Oregon, Pennsylvania, Tennessee, Virginia, Indiana und West Virginia erklärten P.tomentosa daraufhin als invasiv, da die Baumart sowohl Buschfeuer, Planierung mittels Bulldozer als auch direktes Abschneiden ohne Probleme übersteht und nur der direkte Einsatz von Glyphosat bzw. Triclopy auf dem Stubben ein erneutes Austreiben verhindert (Remaley, 2005).

Natürliche und anthropogene Störungen, wie bspw. großflächige Feuerereignisse, fördern die Verbreitung der P. in den Wäldern. Innes (2009) erwähnt die Verwendung von Paulownia zur Rekultivierung von Tagebauten und der schnellen Wiederbegrünung von großen Waldbrandflächen, wobei ein Management der Art nach erstmaligem Ausbringen und selbständiger Verbreitung als äußerst schwierig beschrieben wird. Durch ihr schnelles Höhenwachstum kann sie ab einer Mindesthöhe von 1,5 m mittels ihres Blätterdaches die umliegenden Arten ausdunkeln und somit die Regeneration einer natürlichen Waldgesellschaft stark behindern. (Innes, 2009) 


\subsubsection{Klassifikation}

\subsubsection{Morphologie und Eigenschaften}

\subsection{Allgemeine Morphologie}

Die Borke der Paulownia variiert farblich von Braun über Grau bis hin zu einem leichten Schwarz. Sie ist bei jungen Bäumen glatt und über den gesamten Stamm hinweg mit Lenticellen versehen. Bei älteren Bäumen kommt es durch das rasch voranschreitende Wachstum zu Rissen in der Borke die in Längsrichtung verlaufen.

Die Krone kann bleistiftartige bis regenschirmartige Formen annehmen und wirkt durch die wenigen aber großen Blätter eher licht. Die älteren Äste einmal ausgenommen, sind alle Blattteile der Krone mit feinen Härchen bedeckt.

Im Laufe des Wachstums von der Jungpflanze zum Baum und stark abhängig vom Standort und der Verfügbarkeit von Licht unterscheiden sich die Blätter in Form und Größer voneinander.

Die Blüte ist vergleichsweise groß und zeigt sich in einem Farbspektrum von Weiß bis Lila.

Die Samen sind zweischichtig ummantelt und verfügen so über eine flügelartige Gewebeschicht, die der schnelleren Verbreitung im Wind dienlich ist. Sie entwickeln sich in braunen, anfänglich weichen und später verholzenden Fruchtkapseln, die von außen klebrig behaart sind. (Zhu et al., 1986)

\subsection{Taxonomische Eigenschaften zur Charakterisierung}

Im Laufe der Jahrtausende langen Entwicklungsgeschichte der Paulownia kam es immer wieder zur geographischen Isolation in sich stark voneinander unterscheidenden Klimaregionen. Die Gattung hat sich dabei auf natürlichem Wege an die unterschiedlichen Umwelteinflüsse angepasst und es haben sich verschiedene Arten ausgebildet.

Am einfachsten lassen sich diese Arten bei älteren Bäumen anhand der Größe der Blätter, der Farbe der Blüten und mittels der Dichte der Härchen-Bedeckung voneinander unterscheiden.

Als stabile Charakteristika und als Basis zur Bestimmung ${ }^{6}$ der Arten führen Zhu et al. (1986) die Länge des Blütenstandes, mit oder ohne Pedunculus (Blütenstängel), die Tiefe des Kelches, die Form der Blüte und der Samenkapsel, die Dicke des Perikarps (Fruchtwand) und die Form der Plazenta auf.

Als weitere bestimmende Eigenschaften, die aber ihrer Ansicht nach mit ein wenig Vorsicht zu genießen sind, gelten die Größe der Früchte und der Blüte, die Form und Größe des paniculaten (rispenförmigen) Blütenstandes und die Form der vollentwickelten Blätter an älteren Bäumen.

Mit dem Alter der Bäume variiert die Bedeckung der Blätter mit Härchen in den verschiedenen Kronenabschnitten. Die Kelchblätter und die Behaarung der Blattspreiten zeigen Variationen innerhalb einer Art und sollten daher zur Bestimmung nur als letztes herangezogen werden. (Zhu et al., 1986)

\footnotetext{
${ }^{6}$ Ein Bestimmungsschlüssel mit systematischer, bildlicher Unterstützung ist dem Anhang zum Kapitel I beigefügt (Tab. 8); entnommen und verändert nach Zhu et al. (1986).
} 


\subsubsection{Taxonomie}

\subsubsection{Historische Taxonomie}

In der „Monographie der Paulownia“ von Chen Chu (1061 - 982 v.Chr.) findet sich die erste detaillierte Beschreibung der morphologischen Charakteristika der Art (Zhu et al., 1986). In dem Buch „Die Flora Japans“ von 1781 wird die Baumart von dem schwedischen Botaniker Karl Thunberg erwähnt und der Gattung Bignoniaceae zugeordnet, wobei er die Art Bignonia tomentosa hervorhebt, die gleichzusetzen ist mit der heutigen Bezeichnung P.tomentosa.

Der bayrische Botaniker Joseph G. Zuccarini (1797 - 1848) und der bayrische Arzt, Ethnologe und Botaniker Philipp Franz von Siebold (1796 - 1866) studierten die Baumart noch genauer und ordneten sie 1835 erstmalig der Gattung Scrophulariaceae zu (Flora Japonica, 1835).

Die Fachliteratur, des sich anschließenden Jahrhunderts, trägt reiche Blüte an verschiedenen neuen Arten und Bezeichnungen, wobei der Großteil davon sich bei näherer Betrachtung als Synonyme herausstellt. Erst mit der Monographie von $\mathrm{Hu}$ (1959) wird ein Teil der Namensverwirrungen aufgelöst und es erfolgt eine Zuordnung die sechs Arten umfasst.

Bis heute ist der Streit der Gelehrten über die korrekte Einteilung der Arten und die Zuordnung zu den Familien Bignoniaceae oder Scrophulariaceae nicht vollständig abgeschlossen (Cabi, 2019). Die neuere Literatur favorisiert jedoch in ihrer Mehrzahl die Bildung einer eigenen Familie der Paulowniaceae (Blauglockenbaumgewächse) und eine Einteilung in sieben Arten (eFloras, 2014).

\subsubsection{Heutige Taxonomie}

Zur leichtern Verbildlichung der heutigen Taxonomie bietet sich der Taxonomie-Baum nach Cabi (2019) an:

Domain: Ekaryota

Kingdom: Plantae

Phylum: Spermatophyta

Subphylum: Angiospermae

Class: Dicotyledonae

Order: Scrophulariales

Family: Scrophulariaceae (oder Paulowniaceae)

Genus: Paulownia

Species: ...

Laut Hecker und Weisgerber (2014) und eFloras (2014) sollte die Gattung Paulownia mit sieben Arten wie folgt systematisiert werden:

$\begin{array}{ll}\text { P.catalpifolia } & \text { T.Gong ex D.Y.Hong } \\ \text { P.elongata } & \text { S.Y.Hu } \\ \text { P.fargesii } & \text { Franchet } \\ \text { P.fortunei } & \text { (Seemann) Hemsley } \\ \text { P.kawakamii } & \text { T.Ito }\end{array}$


P.taiwaniana

P.tomentosa
T.W.Hu \& H.J.Chang - Synonym: P.australis Gong Tong natürlicher Hybrid zwischen P.fortunei und P.kawakamii (Wang u. a. 1994)

Thunb. Steudel - (Blauglockenbaum, Kaiser-Paulownie) mit den Varietäten: P.tomentosa var. Tomentosa

P.tomentosa var. Tsinlingensis Thunb. Steudel

Laut Cabi (2019) noch zusätzlich die Varietäten:

Pai Gong Tong
P.tomentosa var. lucida
P.tomentosa var. lanata

Zhu et al. (1986) ergänzen diese Systematik noch um die Art P.albipholea und unterscheiden zwischen P.taiwaniana und P.australis als eigenständige Arten.

Weiterhin betonen die Autoren Zhu et al. (1986) wie wichtig die Unterscheidung der Arten sowohl für den kommerziellen Anbau als auch für die wissenschaftliche Forschung ist und verweisen auf das Ziel der schon frühzeitigen Unterscheidung der Sämlinge und das obwohl sich diese nach eigener Aussage noch stark im Laufe ihres Wachstums sich vom Erscheinungsbild her wandeln.

\subsubsection{Artenliste}

Die Website „International Plant Names Index“ (2020) listet 32 Arten und Hybriden der Gattung Paulownia auf. Die vorab schon dargestellte Systematik soll hier nur mittels einer Aufzählung im Anhang zum Kapitel I (Tab. 9) als Gesamtüberblick ergänzt werden. Zuerst wird dabei in alphabetischer Reihenfolge die Art genannt, dann die Quelle ihrer ersten Erwähnung in verkürzter Form aufgeführt und in Klammern dahinter die Jahreszahl der Veröffentlichung.

\subsubsection{Die „Hauptbaumart“ P.tomentosa}

Die am meisten verbreitete, daher am besten erforschte und umstrittenste P.-Art ist die P.tomentosa. Sie soll beispielgebend für alle anderen Arten im Folgenden etwas näher beschrieben werden.

Sie wird häufig mit Bäumen der Familie Bignoniaceae (Trompetenbaum) und darin bevorzugt mit Catalpa speciosa (Prächtiger Tompetenbaum) verwechselt. Sowohl bei der Baumgröße als auch bei den Blättern und Blüten bestehen große Ähnlichkeiten, jedoch ist die Unterscheidung hinsichtlich der Form und Anzahl der Früchte sehr einfach. Bei Catalpa-Bäumen sind es dünne, ca. $20-50 \mathrm{~cm}$ längliche Schoten und bei der Paulownia kleine gebündelte Kapseln (Abb. 5) (Cabi, 2019) 


\subsubsection{Taxonomie und Verbreitung}

\subsection{Taxonomie}

Die Zusatzbezeichnung "tomentosa“ bezieht sich auf die filzige, beidseitige Behaarung der Blätter, der Blütenstände und der noch jungen Triebe (Hecker and Weisgerber, 2014). Anhand der Behaarung können die Varietäten unterschieden werden, da bspw. die P.tomentosa var. tomentosa im Vergleich zu var. tsinlingensis von der Sprossachse abgewandte (abaxial) stark behaarte Blätter hat (Vor et al., 2015).

Die folgende Taxonomie erfolgt auf Grundlage von Cabi (2019) und Vor et al. (2015):

Familie: $\quad$ Paulowniaceae (Blauglockenbaumgewächse)

(ehemals Familie Scrophulariaceae - Braunwurzgewächse)

Gattung: Paulownia - Siebold et Zucc. (Blauglockenbaum)

Art: $\quad$ P.tomentosa (Thunb. ex Murray) Steud. (1841)

Varietäten: P.tomentosa f. pallida Rehd.

P.tomentosa var. lanata (Dode) Schneid.

P.tomentosa var. lucida Z. X. Chang \& S. L. Shi

P.tomentosa var. tomentosa

P.tomentosa var. tsinlingensis (Pai) Gong Tong

Weitere wissenschaftliche (historische) Namen:

Bignonia tomentosa Thunb. (J. A. Murray, Syst. veg. ed. 14:563. 1784

May-Jun (Fl. jap. 252. 1784 Aug))

Incarvillea tomentosa (Thunb.) Spreng.

P.grandifolia Hort. ex Wettst.

P.imperialis Siebold \& Zucc.

P.recurva Rehd.

P.tomentosa (Thunb.) Siebold \& Zucc. ex Steud.

International gebräuchliche Namen:

China: Maopaotong, Ribenpaotong, Rongmaopaotong, Zihuapaotong, Zitong

Deutschland: Kaiser-Paulownie, Blauglockenbaum

Großbritannien: Chinese empress tree, Princess tree, Empress tree

Paulownia, Foxglove tree, Karri-tree

Frankreich: Paulownia (de Chine), Paulownia impérial, Paulownia tomenteux, Arbré d`anna paulownia

Italien: Paulovia

Japan: Kiri

Niederlande: Anna-paulownaboom 


\subsection{Natürliche Verbreitung}

Die P.tomentosa wurde früher zur Familie der Braunwurzgewächse und darin als einzige baumförmige Art gezählt. Heutzutage wird sie aber der eigenständigen Familie der Paulowniaceae (Blauglockenbaumgewächse) zugeordnet. Ihr natürliches Verbreitungsgebiet (Abb. 6) befindet sich in Zentral-China und Teilen von West-China mit einem Verlauf im Bereich von ca. $28^{\circ}-40^{\circ} \mathrm{n} . \mathrm{Br}$. bis hin zu 105-123 ö.L. (Bean, 1973; Lee, 1983).

Sie ist in Höhenlagen von $500-1.800$ mü.NN zu finden und kommt daher verstärkt in den Provinzen Ganzu, Henan, Hubei, Jiangxi, Liaoning, Shaanxi und Shanxi vor, wobei sich sehr wüchsige Plantagen in Ost-Gansu und im Großraum von Beijing (Peking) befinden (Bean, 1973).

$\mathrm{Da}$ die Art in den letzten zweitausend Jahren verschiedenartig in den Ländern Süd- und Ostasien kultiviert wurde und dabei vor allem in Japan und Korea, ist eine genaue Beschreibung der natürlichen Verbreitung nicht mehr möglich

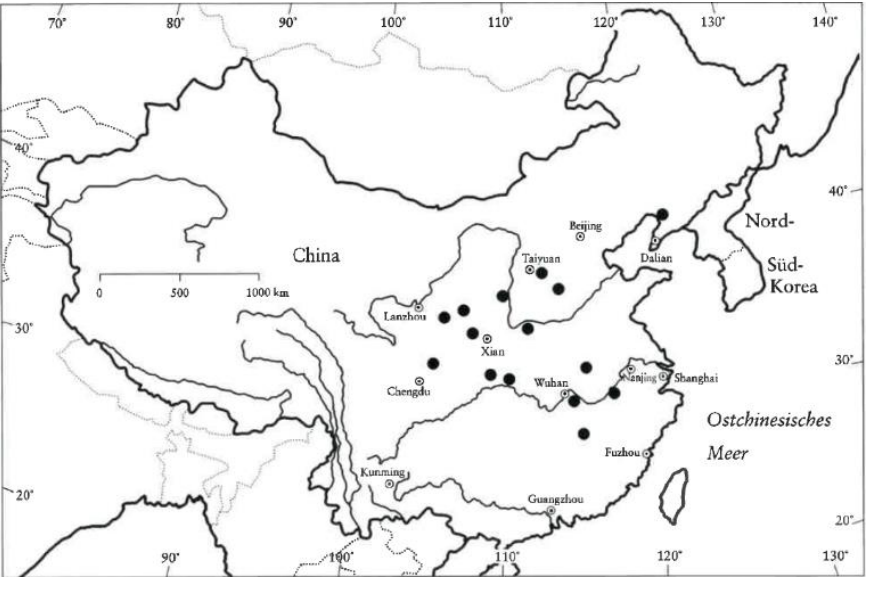

Abb. 6. Verbreitungsgebiet von P.tomentosa, verändert nach Zhu et al. (1991). (eFloras, 2014).

\subsubsection{2 Ökologie}

Von der Baumart werden große Temperaturunterschiede im Bereich von $-20^{\circ} \mathrm{C}$ bis + $40{ }^{\circ} \mathrm{C}$ toleriert. P.tomentosa ist die frostresistenteste aller Arten, wobei auch sie ihr Wuchsoptimum nur bei langanhaltenden Wärmeperioden, ideal wären Tagesdurchschnittswerte zwischen $24-29^{\circ} \mathrm{C}$, erreichen kann.

Sie bevorzugt vollsonnige, windgeschützte Standorte und tiefgründige sandige Lehmböden bis zu gut drainiertem Lösslehm. Auf Böden mit hohem Tongehalt kann sich das Wuchspotential nicht vollständig entfalten, wie Versuche in den USA gezeigt haben (Johnson et al., 2003). Als Pioniergehölz kann P.tomentosa auf Rohböden gedeihen die einen $\mathrm{pH}$-Wert zwischen 4,0 bis 8,5 aufweisen. In natürlichen Mischwäldern kommt sie, als lichtliebende Art, nur mit gleichwüchsigen bzw. wuchsunterlegenen Arten zur Vergesellschaftung, und sie besiedelt dabei Areale mit Jahresniederschlägen von 500 bis $1.500 \mathrm{~mm}$. (Zhu et al., 1986)

\subsection{Arttypische Eigenschaften}

P.tomentosa erreicht im Durchschnitt Höhen von 12 - $15 \mathrm{~m}$, wobei auch Exemplare von bis zu $25 \mathrm{~m}$ bekannt sind. Ab einer Stammhöhe von ca. $2 \mathrm{~m}$ beginnt die Verzweigung über wenige Hauptäste hinweg, die sich etagenartig fortsetzt und im Kroneninneren von Reiterations-Trieben ${ }^{7}$ wiederholt wird und somit das lockere Erscheinungsbild der Gesamtkrone prägen. (Dening, 1937)

${ }^{7}$ Reiteration bezeichnet die Ausbildung von Trieben aus Proventivknospen (schlafenden Knospen), Quelle: (Bartels, 1993) 


\subsection{Triebe, Knospen, Blätter}

Die grünen, filzig behaarten Jungtriebe besitzen große Lenticellen. Das vorwiegend weiße Mark kann bei den Trieben hohl sein, was sich auch bei den ausgewachsenen Bäumen verstärkt beobachten lässt.

Eine Endknospe fehlt, wobei an den Jungtrieben Seitenknospen häufiger auftreten. Die Winterknospen stehen oberhalb der elliptischen bzw. kreisförmigen Blattnarben.

Anders als bei mitteleuropäischen Baumarten üblich, zeigt P.tomentosa keinerlei Verfärbung der Blätter zum Herbst hin. Stattdessen verbleiben diese bis zum ersten Frost an den Ästen hängen. (Hecker and Weisgerber, 2014)

Die Größe der Blätter variiert stark im Laufe der Entwicklung von der Jungpflanze (mehr als $50 \mathrm{~cm}$ in Länge und Breite) zum Baum und erreichen im Durchschnitt $12-30 \mathrm{~cm}$ in Länge und Breite, mit einem 15 - $20 \mathrm{~cm}$ langen Blattstiel. Unterseitig sind eine Nervatur und filzige, graubraune mehrfach verzweigte Haare (Baumhaare „dendritic branched trichomes“ (Hoppe, 2020)) erkennbar. (Hecker and Weisgerber, 2014)

\subsection{Blüten, Früchte und Samen}

Die P.tomentosa blüht normalerweise schon im zweiten Jahr was im Vergleich zu den anderen Arten sehr früh ist, da diese normalerweise erst im fünften bzw. sechsten Jahr Blüten tragen. Der Blühzeitpunk ist zu Beginn der Vegetationsperiode bei den Arten sehr unterschiedlich ausgeprägt und tritt bei der P.tomentosa, noch vor dem Laubaustrieb Ende Mai, vergleichsweise spät auf. Die Knospen der entomophilien ${ }^{8}$ Blüten werden im Jahr zuvor vom Baum gebildet. (Zhu et al., 1986)

Die Niederschlags- und Temperaturverhältnisse im Spätsommer in den Monaten August bis September sind entscheid für die Entwicklung der Knospen, da bei einsetzender Trockenheit die Anzahl vom Baum verringert wird. Diese verströmen einen intensiven Duft und werden von Bienen bestäubt.

Die eiförmigen Fruchtkapseln sind ca. $3-4 \mathrm{~cm}$ lang, dich behaart, anfänglich lederig und bei Kontakt klebrig und verholzen erst später. Die Samen, die sich zu ca. 2000 3.200 Stück in jeder Kapsel befinden, sind ungefähr $4 \mathrm{~mm}$ lang und verbreiten sich anemochor (durch den Wind). Ein ausgewachsener Baum produziert im Durchschnitt bis zu 20 Millionen Samenkörner pro Jahr (Cabi, 2019). Ein Kilo Saatgut enthält geschätzte 4-6 Mio. Samen, bei einem durchschnittlichen Tausendkorngewicht von ca. 0,17-0,25 g. Das Aufplatzen der Kapseln ist deutlich hörbar und erfolgt bei trockenem und vor allem sonnigem Wetter erst Anfang Januar. (Hecker and Weisgerber, 2014)

Die Verbreitung kann sowohl durch Wind als auch Wasser über größere Distanzen bis zu Entfernungen von $3 \mathrm{~km}$ von der Mutterpflanze erfolgen. Die Samen können, insofern sie nicht gleich austreiben, zwischen 2 bis 3 Jahre keimfähig im Boden überdauern. (Innes, 2009)

\subsection{Rinde, Borke und Holz}

Das Holz der P.tomentosa gehört mit $0,3 \mathrm{~g} / \mathrm{cm}^{3}$ zu den leichtesten Hölzern Chinas und schwindet nur bis zu ca. 8,5\% (Mayr, 1906). Die deutlich erkennbaren Jahrringgrenzen des hellbraunen Kernholzes werden von einem dünnen, hellgrauen Splint umgeben, an den sich die graubraune Rinde und die Borke anschließen.

\footnotetext{
8 entomophil bzw. Entomogamie $w$ [von entomo- , griech. gamos = Hochzeit], Entomophilie, Insektenbestäubung, Insektenblütigkeit, die Übertragung des Pollens einer Blüte auf die Narbe einer artgleichen anderen Blüte durch Insekten. Quelle: LexBio (2020)
} 
Nahezu alle Baumstämme weisen ein im Innern zylindrisch durch den Stamm verlaufenden Hohlraum auf. Generell sind die Zweige und Äste windbruchgefährdet, was durch eine reiche Fruchtbildung noch verstärkt werden kann. Die dabei entstehenden Wundstellen zeigen nur eine mäßige Vernarbung und auch eine Überwallung erfolgt nur in geringem Umfang. (Hecker and Weisgerber, 2014)

\subsection{Wurzeln}

Die P.tomentosa besitzt ein gut entwickeltes Wurzelsystem, welches von mehreren unterschiedlich stark ausgeprägten Seitenwurzeln, statt einer Pfahlwurzel, gebildet wird. Sie verlaufen meist senkrecht in die Tiefe und können mit den von ihnen ausgehenden Seitenwurzeln weit über die oberflächliche Kronenprojektion hinausgehen. Die in einem Radius von ca. 0,5-4 m um die Stammmitte herum verlaufenden oberflächennahen Wurzeln sind dicht verzweigt. (Zhu et al., 1986)

P.tomentosa geht eine Symbiose mit Endomykorrhiza ein, die großen Einfluss auf das Höhen-, Durchmesser- und somit das Biomassewachstum haben können (Büker, 2015; Mehrotra, 1996; Mehrotra et al., 1998; Mehrotra, 1997b).

\subsubsection{Waldbauliche und Ertragskundliche Behandlung}

Aufgrund der sehr geringen Schattentoleranz wäre nur nach größeren Störereignissen, wie bspw. nach großflächigen Bränden bzw. Windwürfen, eine Einbringung der Baumart in die Waldgesellschaften Mitteleuropas denkbar. Sie unterliegt nicht dem Vermehrungsgutgesetz und es gibt keine Informationen über die natürliche Variabilität (Vor et al., 2015).

Sehr häufig ist der Anbau in Form von Wertholz- bzw. Kurzumtriebsplantagen auf Ackerflächen zu finden (Cathaia, 2020; Thielen, 2019). Als ergänzende Baumart in Agroforstsystemen wird sie in Kombination mit Feldfrüchten oder als Reinkultur verwendet. (Cabi, 2019)

\subsection{Wachstum, Entwicklung und Ertrag}

Das Längenwachstum von P.tomentosa kann sich im Juli - August auf bis zu $5 \mathrm{~cm}$ pro Tag erhöhen, was erklärt warum ein jährlicher Höhenzuwachs von $4 \mathrm{~m}$ bei einem BHDZuwachs von $5,5 \mathrm{~cm}$ möglich ist. Dieses Wachstum verläuft bis in den beginnenden Herbst hinein, was dazu führt, dass die jüngeren Sprossabschnitte nicht mehr ausreifen und im Laufe des Winters zurückfrieren bzw. absterben. (Zhu et al., 1986)

Das durchschnittlich erreichbare Baumalter wird allgemein mit 60 - 70 Jahren angegeben, unter sehr guten Verhältnissen mit 100 Jahren, wobei die Wuchskraft im Alter zwischen 20 - 30 Jahren kulminiert (Innes, 2009; Kiermeier, 1977). Diese vergleichsweise Kurzlebigkeit kombiniert mit Raschwüchsigkeit, einer großen Samenproduktion und einer hohen Lichtbedürftigkeit sind klare Merkmale eines Pioniergehölzes (Kiermeier, 1977).

In China werden in agroforstlichen Produktionsformen ca. 1,5 Mio. ha mit P.-Arten in Kombination mit Hirse, Mais, Raps, Weizen und verschiedenen Gemüsesorten angebaut (Hecker and Weisgerber, 2014). 
Von Vorteil sind dabei die lockere und daher sehr lichtdurchlässige Krone, der vergleichsweise späte Blattaustrieb und die nur geringe Konkurrenz, im oberflächennahen Durchwurzelungsbereich des Bodens, mit den anderen Nutzpflanzen. Weitere abiotische und biotische Aspekte wie bspw. die Verbesserung der mikroklimatischen Bedingungen und der Frost- und Windschutz bei Reihenpflanzungen, wirken sich positiv auf die Erträge aus. (Kiermeier, 1977)

Daher verwundert es nicht, dass in Mischkulturen eine größere Wüchsigkeit erreicht wird, die bei ca. 10 - jährigen Bäumen mittlere BHDs zwischen $35-40 \mathrm{~cm}$ und eine Holzmasse von 0,5-1,5 $\mathrm{m}^{3}$ pro Baum erzeugen. Auch die Getreideerträge lassen sich auf diesen Flächen um bis zu 23 \% steigern. (Zhu et al., 1991)

Wenn statt ausschließlicher Biomasse auch Wertholz produziert werden soll, so ist aufgrund der sympodialen Verzweigung ein erhöhter Pflegeaufwand nötig (siehe auch 1.1.5.2.2 Kronenwachstum) (Stimm et al., 2013).

\subsection{Verjüngung, Vermehrung und Anzucht}

Die natürliche Verjüngung kann nur auf Freiflächen ohne Konkurrenz von Begleitwuchs bzw. Beschattung anderer Arten erfolgen, da die Sämlinge auf einen hohen Lichtgenuss angewiesen sind.

Generell ist die Dauer der Keimung stark von der über Tage hinweg vorherrschenden Durchschnittstemperatur abhängig. Hecker und Weisgerber (2014) geben bei $30^{\circ} \mathrm{C}$ eine Dauer von 9 Tagen an, welche sich bei niedrigeren Temperaturen auf drei Wochen verlängern kann. Dies erklärt warum P.tomentosa-Keimlinge häufig zwischen Pflastersteinen und Gesteinsritzen zu finden sind, da hier höhere Temperaturen durch den sogenannten Kacheloffeneffekt erreicht werden, was die Konkurrenz durch andere Pflanzen wiederum gering hält.

Das Saatgut lässt sich gekühlt zwei bis drei Jahre aufbewahren und erreicht dann noch immer eine Keimungsrate zwischen 70 - 90 \% (Zhu et al., 1986). Um die Keimung zu beschleunigen empfiehlt sich die Behandlung in einem Wasserbad für 10 Minuten bei 40 ${ }^{\circ} \mathrm{C}$ und dem weiteren Einweichen bei Raumtemperatur für 24 Stunden (Hecker and Weisgerber, 2014).

Bereits ein- bis zweijährige Sämlingspflanzen eignen sich zur vegetativen Vermehrung. Hierbei werden Wurzelstücke mit einer Länge von $15-18 \mathrm{~cm}$ und einer Breite zwischen $1-4 \mathrm{~cm}$ geschnitten. Diese werden dann im Frühjahr aufrecht stehend, ca. einen Zentimeter unter die Bodenoberfläche gesteckt. Es erfolgt in den ersten Wochen ein rasches Wurzelwachstum, und die sich anschließende verspätete Sprossentwicklung bedarf zusätzlicher Pflegemaßnahmen, z.B. dem Ausgeizen (Entfernung von Trieben in den Blattachseln) der unerwünschten Seitentriebe. (Zhu et al., 1986)

Hecker und Weisgerber (2014) erwähnen auch gute Vermehrungs-Ergebnisse bei verschiedenen In-Vitro-Verfahren. 


\subsubsection{Allgemeine Ökologie}

\subsubsection{1 Ökologische Anforderungen}

\subsection{Boden}

Die Tatsache dass die Paulownia über ganz China hinweg Verbreitung findet, ist ein Zeichen dafür, dass sie auf den unterschiedlichsten Bodentypen gedeihen kann.

Die Toleranz gegenüber unterschiedlichen $\mathrm{pH}-$ Werten variiert zwischen den Arten von 4,5 bis 8,5 . Außerhalb dieser Spannweite kann die Gattung überleben, gedeiht dann aber nur mit stark eingeschränktem Wachstum. Auch zeigen sich bei Böden mit einem Salzgehalt von über $1 \%$ Störungen beim Wachstum. (Zhu et al., 1986)

Paulownia kann auch auf sehr armen Böden gute Zuwachsraten erreichen, wobei natürlich eine bessere Nährstoffversorgung für deutliche Steigerungen sorgen kann. Zhu et al. (1986) erwähnen diesbezüglich Experimente mit zusätzlichem Dünger und raten zu Substraten mit hohem Stickstoff-, Phosphat- und Kaliumgehalten.

Für ein gutes und schnelles Wachstum braucht Paulownia ein gut ausgebildetes Wurzelsystem und bevorzugt daher tiefgründige, lockere, feuchte und gut durchlüftete Böden. Daher ist sie am häufigsten auf sandigen und lehmigen Böden zu finden. Sie bevorzugt eine Boden-Porigkeit im Bereich zwischen $10-50 \%$ und eine Bodendurchlüftung von über $30 \%$, bei einer Lagerungsdichte zwischen $1,03 \mathrm{bis} 1,3 \mathrm{~g} / \mathrm{cm}^{3}$. Die Baumart kann nur auf Böden gedeihen deren Grundwasser tiefer als 1,5 m anliegt, da sie gegenüber Staunässe empfindlich ist und schon nach 3 - 4 Tagen abzusterben droht. (Zhu et al., 1986)

Tab. 1: Vergleich der Paulownia Arten anhand von Verbreitungsgebiet, Temperatur, Niederschlag und Bodenverhältnissen, verändert nach Zhu et al. (1986), S. 22.

\begin{tabular}{|c|c|c|c|c|c|c|c|c|c|c|}
\hline \multirow[b]{2}{*}{ Species } & \multicolumn{3}{|c|}{ Distribution } & \multicolumn{3}{|c|}{ Temperature } & \multicolumn{2}{|c|}{ Rainfall } & \multicolumn{2}{|r|}{ Soil } \\
\hline & LAT & LONG & ALT & $\operatorname{Max}$ & Min & Mean & $\begin{array}{l}\mathrm{AN} \\
\mathrm{mm}\end{array}$ & $\begin{array}{c}\text { Dry } \\
\text { months }\end{array}$ & $\mathrm{pH}$ & Texture \\
\hline P. tomentosa & $\mathrm{N} 28-40$ & E105-128 & 1500 & $40^{\circ} \mathrm{C}$ & $-20^{\circ} \mathrm{C}$ & $17-11^{\circ} \mathrm{C}$ & $1500-500$ & $3-9$ & $5-8.5$ & light clay-sandy \\
\hline P. elongata & N28-36 & E112-120 & 1200 & $40^{\circ} \mathrm{C}$ & $-15^{\circ} \mathrm{C}$ & $17-12^{\circ} \mathrm{C}$ & $1500-600$ & $3-9$ & $5-8.5$ & heavy loam-sandy \\
\hline P. catalpifolia & N32-36 & E113-120 & 800 & $38^{\circ} \mathrm{C}$ & $-15^{\circ} \mathrm{C}$ & $15-12^{\circ} \mathrm{C}$ & $1300-700$ & $4-8$ & $6-8.0$ & light clay-sandy \\
\hline P. fortunei & N18-30 & E105-122 & 1100 & $40^{\circ} \mathrm{C}$ & $-10^{\circ} \mathrm{C}$ & $23-15^{\circ} \mathrm{C}$ & $2500-1200$ & $2-3$ & $4.5-7.5$ & light clay-sandy \\
\hline P. taiwaniana & $\mathrm{N} 22-25$ & E120-122 & 1000 & $39^{\circ} \mathrm{C}$ & $2^{\circ} \mathrm{C}$ & $20-23^{\circ} \mathrm{C}$ & $2300-1800$ & $2-3$ & $4.5-7.0$ & light clay-sandy \\
\hline P. albiphloea & $\mathrm{N} 28-30$ & E100-110 & 600 & $41^{\circ} \mathrm{C}$ & $-3^{\circ} \mathrm{C}$ & $18-20^{\circ} \mathrm{C}$ & $900-1400$ & $3-4$ & $4.5-7.5$ & medium clay-sandy \\
\hline P. australis & $\mathrm{N} 22-30$ & E110-122 & 700 & $38^{\circ} \mathrm{C}$ & $-6^{\circ} \mathrm{C}$ & $14-20^{\circ} \mathrm{C}$ & $900-2100$ & $2-3$ & $4.5-7.0$ & light clay-sandy \\
\hline P. kawakamii & $\mathrm{N} 22-30$ & E110-122 & 800 & $38^{\circ} \mathrm{C}$ & $-8^{\circ} \mathrm{C}$ & $14-20^{\circ} \mathrm{C}$ & $1100-2200$ & $2-4$ & $4.5-7.5$ & medium clay-sandy \\
\hline P. fargesii & $\mathrm{N} 23-31$ & E100-110 & 2000 & $34^{\circ} \mathrm{C}$ & $-11^{\circ} \mathrm{C}$ & $13-18^{\circ} \mathrm{C}$ & $1200-1900$ & $1-2$ & $4.5-6.5$ & medium clay-sandy \\
\hline
\end{tabular}

\subsection{Niederschlag}

Eine ausreichende Wasserversorgung während der Vegetationsperiode ist für das schnelle Wachstum der Paulownia nach Meinung von Zhu et al. (1986) von größter Bedeutung. Um dies zu ermöglichen erschließt sich der Baum, mittels Ausbildung eines weitflächigen und auch tiefgehenden Wurzelsystems (Longbrake, 2001), ein großes Bodenvolumen und bildet mit vergleichsweise nur wenigen Blättern einen großen Blattflächenindex aus. 
Die Niederschlagsrate die von Paulownia toleriert wird liegt zwischen 500 bis $3000 \mathrm{~mm}$ pro Jahr (p.a.). Speziell bei dem Minimumwert von $500 \mathrm{~mm}$ p.a. ist es laut Zhu et al. (1986) wichtig, dass der Großteil des Niederschlages während der Warmperiode, also der Zeit der größten Wachstumsrate der Paulownia, fällt.

Die Gattung zeigt keine erhöhte Sensitivität gegenüber Luftfeuchte, wie Versuche in der Sha Che Provinz (Sinkiang Uighur Region) mit künstlicher Bewässerung und einer durchschnittlichen Tagesluftfeuchte von 52 \% gezeigt haben. (Zhu et al., 1986)

\subsection{Wind}

Wind kann, aufgrund der großen Blätter, starke mechanische Kräfte auf die Pflanze bzw. dem Baum ausüben, was leicht mit Schäden, wie bspw. Astabbrüchen an Sämlingen, Jungbäumen und auch noch an ausgewachsenen Bäumen einhergehen kann.

Jedoch spielt der Wind eine entscheidende Rolle bei der Verbreitung der P.-Samen. Diese werden in großer Stückzahl vom Baum produziert. Sie sind klein, leicht und mit einer Gewebeschicht ummantelt, die wie Flügel fungieren und dafür sorgen, dass die Samen zwischen 0,5 - $1 \mathrm{~km}$ vom Mutterbaum verweht werden. (Zhu et al., 1986)

\subsection{Temperatur}

Das Wachstum der Paulownia beginnt ab einem Temperaturwert von $8{ }^{\circ} \mathrm{C}$. Es ist stark von der täglichen Durchschnittstemperatur abhängig, die laut Zhu et al. (1986) im optimalen Bereich zwischen $24-29^{\circ} \mathrm{C}$ liegt und je länger dieses Optimum erreicht wird umso besser ist das Gesamtwachstum.

Wie auch bei anderen Baumarten üblich hat die Paulownia im Laufe ihrer Entwicklung verschiedene Möglichkeiten ausgebildet um Überhitzungen der Gewebeoberfläche bei starken Temperaturschwankungen zu verhindern. Beispielsweise sind die Blütenknospen von dicken, fleischigen Kelchblütenblättern umwickelt, die einen hohen Stärkeund Zuckergehalt aufweisen und somit isolierend wirken. Hinzu kommt eine Ummantelung mit feinen Härchen und Schuppenteilen. Auch die Blätter sind beidseitig von Härchen besetzt, wodurch kleine Luftpolster an der Oberfläche der Epidermis entstehen, die eine isolierende Wirkung entfalten. (Zhu et al., 1986)

Die Arten beenden ihr Höhenwachstum zum Ende der Vegetationszeit bei unterschiedlichen Temperaturen und somit zu verschiedenen Zeiten im Jahr. In China wurde beobachtet dass P.tomentosa dieses bereits im September bei weniger als $20^{\circ} \mathrm{C}$ einstellt. P.elongata stoppt bei $18^{\circ} \mathrm{C}$ zu Oktoberbeginn und P.fortunei etwas später, in etwa zwischen Mitte Oktober und Anfang November. Nur P.kawakamii reagiert erst bei unter $15^{\circ} \mathrm{C}$ Tagesdurchschnittstemperatur mit dem Einstellen des Höhenwachstums. Im Verhältnis dazu verläuft das Dickenwachstum bei allen Arten relativ ähnlich und stoppt erst mit dem Blattfall. (Zhu et al., 1986)

Wie der Tab. 1 zu entnehmen ist, variiert die Kälteresistenz der Arten stark voneinander. Im Allgemeinen wird P.tomentosa als am robustesten beschrieben und widersteht Temperaturen von bis zu $-20^{\circ} \mathrm{C}$. P.elongata (ähnlich P.catalpifolia) toleriert zwischen 15 bis $-18^{\circ} \mathrm{C}$ und P.fortunei (ähnlich P.australis, P.kawakamii und P.fargesii) widersteht noch Temperaturen von - 5 bis $-10^{\circ} \mathrm{C}$. (Zhu et al., 1986) 
Bei einem Freilandversuch im Frühjahr 1976 wurden in Beijing (Peking) alle verfügbaren Arten der Paulownia gepflanzt und beobachtet wie sich die anschließende Winterzeit, mit einer Tiefsttemperatur von - $16^{\circ} \mathrm{C}$, auswirken würde. P.tomentosa hatte erwartungsgemäß keinerlei Schädigungen. P.elongata (ähnlich P.catalpifolia) zeigte auf den sonnigen Standorten nur leichte Frostschäden. Die P.fortunei-Provenienz, aus den Guangzhou / Hongzhou (22-30 $\left.{ }^{\circ} \mathrm{N}\right)$ Gebieten, zeigte eine $100 \%$ Mortalitätsrate, wohingegen die Provenienz der Nanjing-Region $\left(32^{\circ} \mathrm{N}\right)$ mit starken Frostschäden überlebte. Besonders hervorzuheben ist die Beobachtung, dass auch alle Sämlinge von P.fargesii starben und das obwohl diese Art sehr kalte und nasse Klimate in höheren Gebirgsregionen gewöhnt ist. Es wird vermutet, dass der kalte und vor allem trockene Winter des nördlichen Zentralchinas, an den sich die Art nicht anpassen konnte, zu dieser hohen Sterblichkeit beigetragen hat. (Zhu et al., 1986)

Besonders empfindlich sind die Terminalknospen der P.fortunei, weil diese entgegen allen anderen P.-Arten keinen Frostschutz aufweisen. Folglich stirbt im Laufe des Winters ein Großteil der Knospen ab und die Überlebenden bilden sich stark verzweigende Äste im kommenden Frühjahr aus (Zhu et al., 1986), was wiederum erhöhte Pflegekosten zur Erziehung astfreien Stammholzes nach sich zieht.

Natürlich sind die Jungpflanzen mit ihren noch nicht lignifizierten Sprossachsen am schwersten von Frösten betroffen. Die dabei auftretenden Schäden werden hauptsächlich von den großen Temperaturunterschieden zwischen Tag und Nacht verursacht und sind nicht nur eine Folge der Tiefsttemperaturen in der Nacht. An den Schadstellen entsteht Fäulnis und Pilzsporen dringen in das Gewebe ein, was großen Einfluss auf das Baumwachstum und die spätere Holzqualität nimmt. Um dies zu verhindern, ist es in China üblich die Stämme vorab mit nassem Kalk zu bestreichen oder sie mit einer Bandage aus Gräsern zu umwickeln. (Zhu et al., 1986)

\subsection{Licht}

Der Lichtkompensationspunkt der Baumart liegt bei ca. $2.0001 \mathrm{x}^{9}$. Versuche mit P.elongata und P.taiwaniana haben gezeigt, dass der Sättigungspunkt bei ca. $60.000 \mathrm{~lx}$ erreicht wird, Die P.-Arten zeigen unterschiedliche Schattentoleranzen, wobei P.fortunei (ähnlich P.fargesii) am schattenintolerantesten ist, was sich daran zeigt, dass die Verjüngung nur im Offenland auflaufen kann. (Zhu et al., 1986)

Paulownia findet sich in Waldbeständen meist nur einzelstammweise an exponierten Standorten mit großem Lichtangebot, wie beispielsweise entlang von Flüssen oder offenen Tälern. Natürlich entstandene P.-Waldbestände sind daher selten und weisen nur eine geringe Stammanzahl auf bzw. einen niedrigen Bestockungsgrad. Wenn die Baumart in Mischung mit anderen Arten vorkommt, so ist sie bezogen auf die Kronenhöhe zumindest gleichgroß oder größer als die anderen Baumarten.

Sowohl die Keimung als auch das Heranreifen der Samen zu Jungpflanzen erfordert intensiven Lichtgenuss. Laut Zhu et al. (1986) haben Experimente zur Schattentoleranz von Setzlingen gezeigt, dass bereits eine Beschattung von ca. $70 \%$ tödlich auf die Verjüngung wirken kann. Daraus schlussfolgern die Autoren, dass Paulownia eine lichtliebende Art ist, was die Grundlage für ihr schnelles Wachstum ist.

\footnotetext{
${ }^{9} \mathrm{Ix}=$ Lux, Einheit der Beleuchtungsstärke.
} 
Deshalb ist sie ungeeignet für Mischungen mit anderen Baumarten wie ein Experiment in Wu Gueng (Shensi Provinz) zeigte, bei der mittels eines Pflanzverbandes von $3 \times 2 \mathrm{~m}$ P.tomentosa und Populus nigra in Mischung gepflanzt wurden. Die P.tomentosa zeigte im Laufe ihrer Entwicklung nur minderwertiges Wachstum und hatte im sechsten Jahr ihrer Anpflanzung eine Mortalitätsrate von ca. $20 \%$.

Zhu et al. (1986) kommen daher zum dem Schluss, dass Paulownia eine Pionierbaumart ist und sich nicht auf natürliche Weise in Wäldern verjüngen kann, sondern dafür immer Brachen, Kahlschläge oder ehemalige Waldbrandflächen benötigt.

\subsubsection{Wachstum}

In China gibt es bezüglich des P.-Anbaus den Ausspruch: „It looks like a pole in one year, an umbrella in three years and can be sawn into boards in five years!" (Zhu et al., 1986, S.14). Betont werden hierbei das schnelle Wachstum und die damit einhergehende frühzeitige Verfügbarkeit von Stammholz. Tatsächlich erreicht die Baumart im Alter zwischen 20 bis 30 Jahren ihre höchste Wuchskraft und kann dabei in Einzelfällen, bei einem Durchmesser von 1 bis $2 \mathrm{~m}$, bis zu $30 \mathrm{~m}$ hoch werden (Vor et al., 2015). Unter natürlichen Wuchsbedingungen, also in offenen Waldgesellschaften, erreichen die meisten Bäume ein Alter zwischen 60 - 70 Jahren, weswegen die Art als eher kurzlebig beschrieben wird (Innes, 2009; Kiermeier, 1977).

\subsection{Wachstumsraten verschiedener Arten}

Zwischen den P.-Arten gibt es bei gleichen Standortbedingungen unterschiedliche Wachstumsraten. Vergleichsweise am schnellsten wachsen P.elongata, P.fortunei und P.tomentosa, wobei die Unterart P.tomentosa var. tsinlingensis als am langsamsten wachsend beschrieben wird.

In China gibt es für die Landbevölkerung schon seit Jahrzehnten seitens der Regierung die Empfehlung an den „Vier-Wichtigen-Seiten“ P.fortunei zu pflanzen, da diese am ehesten geeignet ist nahe an Gebäuden, um die Dörfer herum und entlang von Straßen und Kanälen zu gedeihen. Zhu et al. (1986) listen zahlreiche Beispiele zum Wachstum auf, worüber die Tab. 2 einen Überblick gibt.

Tab. 2: Vergleich des Wachstums von 5-jährigen Bäumen der Arten P.elongata, P.tomentosa, P.fortunei, P.glabrata aus der Wu Gueng Region (südlich des Yangtze-Flusses, Lat. N34²3’ Long. $\left.E 107^{\circ} 42^{\prime}\right)$, verändert nach Zhu et al. (1986), S. 15.

\begin{tabular}{|c|c|c|c|c|c|c|c|c|}
\hline \multirow[b]{2}{*}{ Species } & \multicolumn{2}{|c|}{ d.b.h. (cm) } & \multicolumn{2}{|c|}{ Height (m) } & \multicolumn{2}{|c|}{ Timber volume } & \multicolumn{2}{|c|}{$\begin{array}{c}\text { Percentage disease } \\
\text { affected }(\%)\end{array}$} \\
\hline & Average & $\begin{array}{c}\text { Annual } \\
\text { increment }\end{array}$ & Average & $\begin{array}{c}\text { Annual } \\
\text { increment }\end{array}$ & $\begin{array}{l}\text { Average } \\
\left(\mathrm{m}^{3}\right)\end{array}$ & $\begin{array}{l}\text { Increase } \\
(\%) \\
\text { Index } \\
(P . \text { glabrata } \\
=100)\end{array}$ & $\begin{array}{c}\text { Witches } \\
\text { broom } \\
\text { disease }\end{array}$ & Sunscald \\
\hline P. elongata & 18.0 & 3.6 & 11.1 & 2.2 & 0.1191 & 275 & 10 & 0 \\
\hline$P$. tomentosa & 15.3 & 3.0 & 9.5 & 1.9 & 0.0716 & 165 & 30 & 10 \\
\hline P. fortunei & 14.7 & 2.9 & 9.3 & 1.9 & 0.0696 & 161 & 0 & 0 \\
\hline P. glabrata & 12.9 & 2.6 & 7.9 & 1.6 & 0.0433 & 100 & 50 & 40 \\
\hline
\end{tabular}




\subsection{Kronenwachstum}

Die Krone eines Baumes ist die Summe vom jährlichen Wachstum der Äste. Im ersten Jahr der Pflanzung entwickelt sich bei der Paulownia nur eine im Durchschnitt ca. $1 \mathrm{~m}$ hohe Krone, da der überwiegende Teil der Wuchskraft in die Ausbildung des unterirdischen Wurzelsystems investiert wird (Longbrake, 2001).

Frühestens $a b$ der zweiten Vegetationsperiode erreicht die Baumart ein durchschnittliches Höhenwachstum von 1,5 bis $2 \mathrm{~m}$ pro Jahr. Erst ab dem dritten bzw. vierten Jahr bilden sich dann aus den seitlichen Stammknospen Äste, die wiederum noch in der gleichen Vegetationsperiode weitere Seitenäste ausbilden und somit eine Zwei-EtagigeKrone entstehen lassen. Auch in älteren Bäumen lässt sich dieses Verzweigungsmuster wiederfinden. (Zhu et al., 1986)

Das Apikal und meist auch die zweiten und dritten axillären (in der Blatt-Achsel) Knospen erleiden, abhängig von der Region in die der Baum wächst, frostbedingte Schäden, wodurch erst die vierten bzw. fünften axillären Knospen zu den neuen Ästen heranreifen. An diesen Ästen bilden sich dann die Knospen für das nächste Jahr, von denen auch wieder die obersten aller Wahrscheinlichkeit nach geschädigt werden und so wiederholt sich die Wachstumsreihenfolge bei den Ästen Jahr für Jahr. Zhu et al. (1986) empfehlen auf Grundlage dieser Beobachtung die Bestimmung des Baumalters durch einfaches Abzählen der Astanzahl.

Die Krone der Paulownia ist sympodial ${ }^{10}$ und das obwohl sie aufgrund des schnellen Höhenwachstums und den dabei vergleichsweise sich nur langsam ausbildenden Seitenästen monopodial ${ }^{11}$ erscheint.

\subsection{Wurzelwachstum}

Das Wachstum der Wurzelsystems wird maßgeblich von den Bodeneigenschaften, den verfügbaren Nährstoffen und der Tiefe, in der das Grundwasser ansteht, beeinflusst. Durch geotrop orientierte Wurzeln erschließt der Baum Tiefen von 0,8-1,0 m, wobei keine Hauptwurzel gebildet wird. (Vor et al., 2015)

Die Autoren Zhu et al. (1986) beschreiben beispielhaft an einem adulten P.elongataBaum wie sich ca. 70 - $85 \%$ seiner Absorptionswurzeln in einem Radius von $40-100$ $\mathrm{cm}$ um den Baum herum verteilen. Sie verweisen darauf wie vorteilhaft eine solche natürliche Wurzelentwicklung für den Anbau der Baumart bei Agroforstsystemen (Intercrooping) ist. In einem anderen Experiment aus der Honan Provinz wurde das gesamte Wurzelsystem einer P.elongata freigelegt und es zeigte sich dass sich die Wurzeln über einer Fläche von 29,5 x 28,1 m ausdehnten, was dem 2,8 fachen der Überschirmung durch die Krone entsprach. (Zhu et al., 1986)

\footnotetext{
10 Sympodial bzw. Sympodium $s$ [von sym- , griech. podion = Füßchen; Adj. sympodial], Scheinachse, Bezeichnung für Stämme, Äste und Zweige, die nicht durch ein gefördertes Wachstum der jeweiligen Mutterachse entstehen, sondern dadurch, dass jeweils ein Seitenzweig das Wachstum verstärkt fortsetzt und die Abstammungsachse übergipfelt, die Abstammungsachse ihrerseits im Wachstum zurückbleibt. Das dadurch entstehende Verzweigungssystem nennt man Monochasium. Solche Sympodien können einer echten Hauptachse sehr ähnlich sehen - vor allem, wenn die Scheinachse das Achsenwachstum gerade fortsetzt und die unterdrückte Abstammungsachse seitlich abgedrängt wird. Quelle: LexBio (2020).

${ }^{11}$ Monopodial bzw. Monochasium s [von mono- , griech. chasis = Spaltung], Form der Verzweigung bei sympodialen Sproßsystemen mit gegenüber der Abstammungsachse geförderten Seitensprossen (Seitenachse). Im Gegensatz zum Dichasium setzt beim Monochasium nur jeweils 1 Seitenzweig die Verzweigung fort. Dabei übergipfelt er seine Abstammungsachse (Telomtheorie), und es entsteht ein Verzweigungssystem mit einer Scheinachse (Sympodium), die sich aus Seitensprossen verschiedener Ordnungen aufbaut. Zeigt diese Scheinachse ein gerades Wachstum, weil die ihre Entwicklung einstellenden Enden der jeweiligen Abstammungsachsen zur Seite gedrängt werden und somit Seitenzweige vortäuschen, so ist dieses sympodiale System kaum von einem monopodialen (Monopodium) zu unterscheiden. Quelle: LexBio (2020)
} 


\subsubsection{Durchmesserwachstum}

Seit den 1970er Jahren gibt es in China eine ganze Reihe von wissenschaftlich begleiteten P.-Anbauten. Auf Grund der Wachstumseigenschaften wurden dabei am häufigsten die Arten P.elongata und P.fortunei gepflanzt. (Hecker and Weisgerber, 2014; $\mathrm{Hu}, 1959$; Zhu et al., 1991, 1986)

\subsection{Durchmesserwachstum am Bsp. P.elongata}

Die Krone der P.elongata bildet sich erst ab der zweiten bzw. dritten Vegetationsperiode heraus. Alle zwei bis vier Jahre zeigen sich Wachstumsschübe des Stammes die ein unregelmäßiges Höhenwachstum zur Folge haben und von Mal zu Mal geringer ausfallen.

Im Alter zwischen 8 - 13 Jahren erreicht der Durchmesserzuwachs seinen Kulminationspunkt. Bei Bäumen aus vegetativer Vermehrung (Wurzelstecklingen) kann dies bereits schon im Alter zwischen $4-8$ Jahren beobachtet werden. Der jährliche Holzzuwachs ist stark von den Bodenverhältnissen abhängig und sinkt bei mäßigen Bedingungen ab dem Alter 14 - 15 Jahren und bei guten erst ab ca. 20 Jahren. (Zhu et al., 1986)

Die Autoren Zhu et al. (1986) konnten durch Analyse einer Vielzahl von P.elongataAnbauten die folgenden Grundsätze ermitteln:

- Das Holzvolumen des Stammes entspricht ca. 70 \% der gesamten Holzmasse des Baumes.

- Der erste Wachstumsschub des Stammes bildet ca. $23 \%$ des gesamten Holzvolumens, wobei der zweite Schub nur $4 \%$ des Holzvolumens generiert. Da sich mehr als $90 \%$ des gesamten Holzvolumens des Baumes aus dem Basisstamm und dem ersten Wachstumsschub entwickeln, raten die Autoren dazu besondere Sorgfalt auf die Pflege der Baumart im Jugendstadium zu legen.

\subsection{Durchmesserwachstum am Bsp. P.fortunei}

Die P.fortunei bildet schon sehr früh meist nur zwei pseudo-dichotome ${ }^{12}$ Äste aus. Vom stärkeren der beiden Äste entwickelt sich die Krone weiter. Das Höhenwachstum ist anfänglich sehr stark, verläuft gleichmäßig von Jahr zu Jahr und erfolgt entlang eines Hauptastes. Der Kulminationspunkt des Holzzuwachses erfolgt später als bei der P.elongata und wird häufig erst nach ca. 15 Jahren erreicht.

Bei gleichem BHD ist das Volumen des Stammholzes bei P.fortunei um ca. $18-36 \%$ größer als bei P.elongata. (Zhu et al., 1986)

Generell zeigen Plantagenbäume einen deutlich schnelleren Holzzuwachs als natürliche in Wäldern gewachsene Exemplare. Dabei zeigen generativ vermehrte Bäume einen deutlich späteren Kulminationspunkt beim Holzzuwachs als vegetative und der Rückgang dieses Wachstums zieht sich über einen deutlich längeren Zeitraum hinweg. (Zhu et al., 1986)

\footnotetext{
12 Dichotom = Verzweigung der Sprossachse in zwei annähernd gleiche Teile .
} 


\subsubsection{Abiotische Risiken}

\subsubsection{Allgemeine abiotische Risiken}

Die Gattung Paulownia kann in einem großen Spektrum an verschiedenen Standortbedingungen gedeihen. Die Samenkeimung wird jedoch in trockenen und sehr sauren Böden, die einen pH-Wert von unter 4,0 aufweisen, stark behindert (Innes, 2009). Auch ein hoher Tonanteil verringert die Überlebensfähigkeit der Jungpflanzen (Johnson et al., 2003).

Altbäume können Tiefsttemperaturen von unter $-20{ }^{\circ} \mathrm{C}$ ohne Schäden überstehen. Jungpflanzen hingegen und noch nicht verholzte Triebe sind durch Früh- bzw. Spätfröste gefährdet und können absterben. Von den verschiedenen P.-Arten besitzt P.tomentosa die größte Frost- und Winterhärte, was laut Stimm et al. (2013) maßgeblich zu ihrer Verbreitung in Europa beigetragen hat.

Aufgrund der großen Blätter besteht auf windexponierten Standorten die Gefahr der gegenseitigen mechanischen Beschädigung. Auch schränkt dies das Wachstum ein (Richter and Böcker, 2001) bzw. erschwert das Heranziehen von geraden und langschaftigen Stämmen und somit die Erzeugung von Wertholz.

Laut Innes (2009) werden bei Feuer die oberirdischen Pflanzenteile abgetötet und die Samen können Temperaturen von über $100^{\circ} \mathrm{C}$ nicht überstehen. Jedoch bilden sich im Anschluss an großflächige Brandereignissen, wie sie in Nordamerika zu finden sind, ideale Wachstumsbedingungen und somit ein hohes Invasivitätspotential der Baumart (Innes, 2009; Johnson et al., 2003).

Die Konkurrenz mit sich schnellentwickelnder Begleitvegetation um die Lichtverfügbarkeit kann dabei zu Wuchshemnissen bzw. zum Absterben der Sämlinge führen (Innes, 2009). Wenn sich die Jungpflanzen etabliert haben sind die frischen Blätter jedoch anfänglich noch empfindlich gegenüber Sonnenbrand (Stimm et al., 2013; Zhu et al., 1986).

\subsubsection{Biotische Risiken}

Natürlich wird die Gattung Paulownia regelmäßig bspw. von Spechten und Schildläusen heimgesucht. Die Stärke des Befalls mit Schaderregern und weiteren Krankheiten ist aber von der Region und somit vom Klima abhängig.

Im Süden von China werden die Bäume hauptsächlich von blattfressenden Insekten und Borkenkäfern wie z.B. dem Bockkäfer (Cerambycidae) befallen. Im Norden sind blattfressende Insekten ebenfalls sehr häufig jedoch ist die Hexenbesen-Krankheit weit problematischer. (Mehrotra, 1997b; Zhu et al., 1986)

\subsubsection{Hexenbesen-Krankheit}

$\mathrm{Zu}$ den schwerwiegendsten Krankheiten bei der Paulownia gehört Paulowniawitches'broom (PWB - die Hexenbesen-Krankheit). Sie ist in China vorwiegend in den nördlichen Provinzen zu finden, verbreitet sich aber mit dem Anbau von Plantagen immer weiter in Richtung Süden. Sie wird durch Phytoplasmen verursacht und beeinträchtigt sehr stark das Wachstum der Bäume und wirkt tödlich, sowohl auf Sämlinge als auch auf Jungpflanzen. (Cabi, 2019; Zhu et al., 1986) 


\subsection{Symptome}

Die Anzahl und Stärke der Symptome dieser Krankheit variieren zwischen den Arten und sind meistens an den Ästen, den Stämmen, den Blüten und auch den Wurzeln zu finden. Bei infizierten Ästen sprießt eine große Anzahl von Knospen und formt ganze Zweigbündel, dessen Erscheinungsbild den Namen der Krankheit begründet. Die Äste fallen im Winter nicht vom Baum, können aber evtl. austrocknen bzw. binnen ein, zwei Jahren absterben.

Die sich ausbildenden Blätter sind klein und dünn und zeigen eine gelbe Blattspreite. Ihre Dichte an Chlorophyll und Proteinen ist um bis zu $30-40 \%$ verringert.

Generell führt die Krankheit zu metabolischen Störungen im physiologischen Stoffkreislauf, was zur Unterversorgung ganzer Kronenabschnitte führt und schließlich das Absterben des Baumes zur Folge hat. (Zhu et al., 1986)

Sämlinge sind im Durchschnitt nur zu ca. $5 \%$ betroffen. Bei Jungpflanzen kann die Krankheit ca. 5 - 30\% befallen und bei Jungbäumen im Alter zwischen $4-5$ Jahren kann sich die Befallsrate auf ca. 50 - 80\% erhöhen. Da infizierte Bäume deutlich kürzere Wurzeln ausbilden, wird das Wachstum von jungen Bäumen stark geschädigt und das der älteren Bäume verringert sich im Mittel um ca. 20 - 25 \%. (Zhu et al., 1986)

\subsection{Infektion}

Die Krankheit wird hauptsächlich durch Baumwanzen (Halymorpha picus) verbreitet (Vor et al., 2015), aber auch andere Insekten wie bspw. Empoasca flavescens (Gattung: Kleinzikaden, Familie: Cicadellidae, Unterfamilie: Typhlocybinae) tragen dazu bei.

Die Hauptverbreitung erfolgt aber durch verunreinigtes Pflanzmaterial (Wurzelstecklinge) bzw. bei der Veredelung (Pfropfen) älterer Bäume. Die Samen befallener Bäume sind, soweit bekannt, von der Krankheit nicht betroffen und können der vorgesehenen Verwendung ohne Einschränkungen zugeführt werden. (Zhu et al., 1986)

\subsection{Maßnahmen zur Bekämpfung}

Als einfachste Möglichkeit um die weitere Ausbreitung der Krankheit zu verhindern, empfehlen Zhu et al. (1986) die Entnahme von stark befallenen Bäumen und deren Beseitigung in Form von Verbrennung.

Wenn nur einzelne Äste betroffen sind, so sollten diese abgeschnitten und die Wundstelle mit einer Salbe aus Terramycin (Antibiotikum, Gruppe: Tetracycline) und Vaseline (im Verhältnis $1 \mathrm{zu}$ 9) bestrichen werden. Die Salbe hilft bei der Wundheilung und verschließt das Pathogen im Baum, wodurch die weitere Verbreitung vermindert wird.

Die Ästungsmaßnahme sollte im Sommer oder frühen Herbst erfolgen, in der das Hauptwachstum der Krone stattfindet, um das Mitwandern des Erregers im Stoffkreislauf des Baumes zu verringern. (Zhu et al., 1986)

Da die Hauptverbreitung der Krankheit über kontaminierte Wurzelstecklinge erfolgt, wird von Zhu et al. (1986) zu einer ganzen Reihe von Schutzmaßnahmen geraten.

Die Stecklinge können zur Desinfektion für ca. 20 Minuten in $45-48{ }^{\circ} \mathrm{C}$ warmes Wasserbad getaucht werden. Statt des Wassers kann auch eine Lösung aus Antibiotika verwendet werden. 
Als ökonomisch sinnvoll und gleichzeitig sehr effektiv wird auch das Tauchen der Wurzelstecklinge für $6-12$ Stunden in einer Lösung aus Bor $(0,01-0,5 \%)$, Natriumthiosulfat $(2 \%)$, Natriumsulfat $(1 \%)$ und Zinksulfat $(0,1 \%)$ und ein sich anschließendes ein- bis zweitägiges Trocknen in der Sonne vor der Pflanzung, beschrieben.

\subsubsection{Anthraknose-Krankheit}

Die Anthraknose (Brennfleckenkrankheit) befällt die Triebe und Blätter von Setzlingen am stärksten, woraufhin diese absterben bzw. abfallen (Cabi, 2019). Ihre Verbreitung wird stark von den klimatischen Bedingungen - Häufigkeit von Niederschlagsereignissen, Luftfeuchtigkeit, usw. - bestimmt. Ausgelöst wird die Krankheit durch einen Erreger im Boden, in diesem Falle einen Ascomyceten (Schlauchpilz) (Butin, 2011).

Bei ca. $25{ }^{\circ} \mathrm{C}$ und einer Luftfeuchte von $90-100 \%$ liegt das Optimum zur Keimung der Paulownia-Samen. Die Infektionsrate erhöht sich aber umso mehr, wenn die Keimlinge zu nahe beieinander gepflanzt sind, sie zu stark beschattet werden, ihre Versorgung mit Frischluft unzureichend ist, bzw. das Pflanzsubstrat dauerhaft zu nass ist oder die Samen zu klein bzw. zu schwach sind. (Zhu et al., 1986)

\subsection{Symptome}

Anzeichen der Krankheit finden sich sowohl an den Blättern, den Blattstielen als auch an den frischen Trieben der Setzlinge. Es bilden sich auf den Blättern ca. $1 \mathrm{~mm}$ große anfänglich bleiche Flecken, die später ins Braune übergehen und dann von gelb-grüner Blattmasse umgeben sind. Diese zuerst befallenen Blätter werden vom Baum verfrüht abgeworfen und die Nachfolger zeigen deutliche Verformungen entlang der Blattspreite. Auf den Blattstielen und den Austrieben finden sich anfänglich kleine, leicht braune Flecken, die sich im Laufe ihrer Entwicklung elliptisch verformen und in vertikaler Richtung über die Pflanzenteile hinweg ausbreiten. Besonders nach stärkeren Regenfällen oder auch in sehr feuchtem Klima erscheinen nach kurzer Zeit Konidien (Pilzsporen) in Form von roten manchmal auch schwarzen Punkten. (Zhu et al., 1986)

\subsection{Maßnahmen zur Bekämpfung}

Ungefähr drei bis fünf Tage vor der Aussaat empfehlen Zhu et al. (1986) die Behandlung der Baumschulböden mit $8 \mathrm{~g}$ Pentachlornitrobenzol (Benzolderivat, keine Zulassung als Pflanzenschutzmittel in der EU) pro $\mathrm{m}^{2}$ und Eisensulfat. Auch sollten die Samen vorab für 30 Minuten in einer 0,2 \% Phenylquecksilberacetat (Organoquecksilberverbindung, keine Zulassung als Desinfektionsmittel in der EU) getaucht werden. Das regelmäßige Versprühen (zweimal im Monat) der Bordeaux-Mixtur (Kupfersulfat + gelöschten Kalk) in der ersten Hälfte der Vegetationsperiode hat sich ebenfalls als sehr hilfreich herausgestellt.

Generell sollten Baumschulen einen Mindestabstand zu Plantagen einhalten und nach Ausbruch der Krankheit geschlossen werden. Auch die Zeit der Aussaat darf sich nicht mit der Regensaison überschneiden, und es sollten nur kräftige, große Samen verwendet werden. (Zhu et al., 1986) 


\subsubsection{Sphaceloma-paulowniae-Hara}

Diese sehr häufige Krankheit befällt sowohl Setzlinge als auch junge Bäume und ihr Auftreten und ihre Verbreitung wird, ähnlich der Anthraknose-Krankheit, stark von klimatischen Bedingungen beeinflusst (Zhu et al., 1986). Es kommt zu Blatt- und Triebschäden, was beträchtliche Zuwachsverluste zur Folge hat und sogar zum Absterben der ganzen Pflanze führen kann (Cabi, 2019; Hecker and Weisgerber, 2014).

Auf den Blättern und manchmal auch an den jungen Stämmen entstehen braune Flecken mit gelben Rändern, die sich später zu Löchern ausbilden. Blätter die sich erst nach der Infektion herausbilden zeigen starke Verformungen.

Die Löcher erweitern sich in Streifenform und führen so zu sich kringelnden Trieben, die bei starkem Befall durch Austrocknung absterben. Die Maßnahmen zur Bekämpfung gleichen sich mit denen der Anthraknose-Krankheit. (Zhu et al., 1986)

\subsubsection{Schwäche-Krankheit bei Jungpflanzen}

Die Symptome dieser Krankheit die hauptsächlich nur Jungpflanzen befällt, sind Wurzel, Stamm-, Blatt- und Knospenfäulnis. Die Haupterreger sind Rhizoctonia solani (asexuelle Form von Thanatephorus cucumeris (Ständerpilz)) und Fusarium spec. (Schlauchpilz).

Die Pathogene überdauern außerhalb der Vegetationsperiode an Pflanzenteilen im Boden und beginnen ihre Verbreitung sobald Tauwetter einsetzt. In Abhängigkeit von den Eigenschaften der Böden und der Form der Verbreitung unterscheidet sich die Schwere der Infektionen. (Zhu et al., 1986)

Als Bekämpfungsmethode wird auch hier von Zhu et al. (1986), in etwa eine Woche vor der Aussaat, die Behandlung des Saatbetts mit dreiprozentigem Eisensulfat mit einer Menge von 4,5 kg / m² empfohlen. Eine regelmäßige Ausbringung der Bordeaux-Mixtur sollte auch hier in Abständen von ca. 10 - 15 Tagen erfolgen.

\subsubsection{Liste weiterer Pilzkrankheiten}

In China kann die Paulownia von einer ganzen Reihe von weiteren Pilzkrankheiten befallen werden:

- Phyllactinia imperialis Miyabe

- Uncinula clintonii Peck

- Cercospora paulowniae Hori

- Mycosphaerella paulowniae Shirai et Hara (Ascomycota, Schlauchpilz)

- Valsa paulowniae Miyabe et hemmi

- Septobasidium tanake (Miyabe) Boed et Steinm.

(Basidiomycota, Ständerpilz)

Laut Zhu et al. (1986) kann die Häufigkeit des Auftretens und der Schweregrad der Infektionen durch die ausschließliche Verwendung von gesunden und wachstumsstarken Bäumen zur Aufforstung verringert werden. 


\subsubsection{Liste weiterer Schädlinge}

Der Schaden der von Insekten ausgeht, variiert in China stark in den Anbauregionen. Weitere bekannte Arten die bei größerem Befall die Baumart Paulownia schädigen können, sind nach Zhu et al. (1986):

- $\quad$ Agrotis ipsilon (Rott.)

- Agrotis toxionis Butler
(Noctuidae, Eulenfalter)

(Noctuidae, Eulenfalter)

Die Imagines der Agrotis fressen die Keimlinge und befallen auch Jungpflanzen und können so zu Wurzelfäule führen (Cabi, 2019).

- Euxoa egetum Schiff

(Noctuidae, Eulenfalter)

- Clavigralla orientalis serica Dolling

(Serica orientalis Matsch),

(Coreidae, Randwanze)

- Anomala corpulenta Matsch

(Scarabaeidae, Blatthornkäfer)

- Anomala ypsilon

(Scarabaeidae, Blatthornkäfer)

- Holotrichia diomphalia

(Scarabaeidae, Blatthornkäfer)

- Gryllotalpa unispina Saussure

(Gryllotalpidae, Maulwurfsgrille)

- Gryllotalpa africana palisot de Beauvois (Gryllotalpidae, Maulwurfsgrille)

- Empoasca flavescens (Fabricius)

(Cicadellidae, Zikade)

- Cicadalla virdis $L$.

(Cicadellidae, Zikade)

- Cryptotothlea variegata Snellen

- Psilogramma menephron Cramer

- Basiprionota bisignata Boh.

- Batocera horsfieldi Hope

- Megopis sinica White

- Thylactus simulans

(Sphingidae, Schwärmer)

(Chrysomelidae, Blattkäfer)

(Cerambycidae, Bockkäfer)

(Cerambycidae, Bockkäfer)

(Cerambycidae, Longhornkäfer)

Der Befall mit Longhornkäfer (Thylactus) führt zu starker Holzentwertung (Cabi, 2019).

Zwei Arten der Gattung Loranthaceae (Mistel, Riemenblume) Loranthus parasiticus (Linn.) Merr. und Loranthus yadoriki Sieb. (Koreanische Mistel) erzeugen Schäden an den Stämmen der Bäume (Zhu et al., 1986).

In Nordamerika konnte auch der Verbiss durch Weißwedelhirsche nachgewiesen werden. Die Baumart wird dadurch zwar in ihrem Wachstum gehemmt, aber aufgrund ihres schnellen Wiederaustriebes nicht gefährdet. (Longbrake, 2001)

Das Buch „Invasiv Plants of Asian Origin Established in the United States and Their Natural Enemies, Volume 1“ von Hao et al. (2004) listet weitere Pilze und Arthropoden auf die nachweislich die Paulownia besiedeln. Die vorab schon dargestellte Aufzählung soll hier nur mittels einer Tabelle im Anhang zum Kapitel I (Tab. 10) als Gesamtüberblick ergänzt werden. 


\subsection{Vermehrung}

Da Paulownia eine Lichtbaumart ist, kann sie sich nicht in geschlossenen Wäldern vermehren und ist daher in der Natur nur in Offenlandflächen bzw. entlang von Flüssen zu finden. Diese natürliche Verbreitungsform führt zur Isolation von Populationen und somit im Laufe der Zeit zur Ausbildung von verschiedenen Arten, die an den gegebenen Umweltbedingungen unterschiedliche Formen der Anpassung zeigen.

Zhu et al. (1986) berichten von einer Untersuchung an einer natürlichen P.fortuneiPopulation bei der einzelne Bäume bis zu $40 \%$ mehr Holzzuwachs zeigten und daher als Samenbäume beerntet wurden, um mit ihrer Genetik weiterzüchten zu können.

Generell wird die Gattung Paulownia als sehr geeignet sowohl zur generativen als auch vegetativen Vermehrung beschrieben. Beispielsweise zeigt die Nachkommenschaft bei Kreuzungen zwischen P.tomentosa und P.fortunei die gewollten Vorteile beider Arten wie z.B. schnelles Wachstum bei gleichzeitig hoher Frostresistenz.

Entscheidend bei der Züchtung zwischen den Arten ist der Blühzeitpunkt, der in warmen Gebieten früher im Jahr einsetzt und unterschiedlich lange anhält. Beispielsweise ist P.fortuneibereits im April verblüht bevor die Blüten von P.tomentosa sich im Mai öffnen. Es bedarf hier folglich einer Ernte des Pollens der bei einer Lagerung bei Raumtemperatur $\left(15-20^{\circ} \mathrm{C}\right)$ sechs Monate haltbar ist und bei dauerhaft unter $0^{\circ} \mathrm{C}$ auch ein Jahr später noch verwendet werden kann. Generell ist eine Vermehrung über Samen, Wurzelstecklinge die aus den Wurzeln geschnitten werden oder über Ast- bzw. Stammstecklinge möglich. (Zhu et al., 1986)

\subsubsection{Generative Vermehrung}

\subsubsection{Samen}

Die Anzahl der sehr kleinen Samen pro Fruchtkapsel variiert stark im Bereich zwischen wenigen Hundert bis mehreren Tausend. Ungefähr ein Kilogramm frischgeernteter Samen enthält 4 - 6 Millionen Samenkörner (ca. 1000 Stück wiegen zwischen 0,17 $0,25 \mathrm{~g}$ ).

Da die Sämlinge ein sehr schnelles Wachstum zeigen, können sie noch im selben Jahr inrer Aussaat von der Baumschule aus ins Offenland verpflanzt werden. Darüber hinaus bietet die generative Vermehrung Vorteile wie bspw. die Ausbildung eines stärkeren Wurzelgeflechts, geringere Anfälligkeit gegenüber Krankheiten und die Jungpflanzen wachsen meist schneller. (Zhu et al., 1986)

\subsection{Samenernte}

Die Autoren Zhu et al. (1986) raten dazu nur Bäume zu beernten, die über 8 Jahre alt sind, einen geraden Stammverlauf ausgebildet haben, gutes Wachstum und keinerlei Krankheiten zeigen und darüber hinaus angepasst sind an die vorhandenen Klimabedingungen.

Die Reife der Früchte unterscheidet sich zwischen den Arten und tritt bspw. bei P.elongata bereits Ende September ein und bei P.fortunei erst Ende Oktober. Bei einer $z u$ frühen Beerntung ist die Keimungsrate zu gering und bei einer zu späten gehen zu viele Samen durch die bereits offenen Fruchtkapseln verloren. 
Die gesammelten Samen sollten in der Sonne für ca. fünf Tage luftgetrocknet werden und können anschließend in Säcken verpackt, kühl, trocken und gut belüftet gelagert werden.

Die Keimungsrate beträgt nach einem Jahr der sachgemäßen Lagerung ca. zwischen $70-90 \%$ und verringert sich nach drei Jahren auf $30-90 \%$ was individuell abhängig von der Baumart ist.

\subsection{Saatvorbereitung}

Zhu et al. (1986) empfehlen zur Vorbereitung der Aussaat die Samen anzufeuchten und sie für 10 Minuten in $40{ }^{\circ} \mathrm{C}$ warmes Wasser zu tränken. Anschließend sollen sie für 24 Stunden mit zimmerwarmem Wasser bedeckt sein und dann ein bis zwei Stunden lang trocknen. Nach circa vier Stunden Trocknungszeit bei $25-35{ }^{\circ} \mathrm{C}$ öffnet sich der Samenmantel und die Keimwurzel bildet sich aus. Nach weiteren fünf bis sechs Tagen sind die Samen zur Aussaat bereit.

Bei ca. $25^{\circ} \mathrm{C}$ Durchschnittstemperatur und einer Luftfeuchte von $90-100 \%$ liegt das Optimum zur Keimung der Paulownia-Samen.

\subsection{Aussaat im Freiland}

Pro Quadratmeter Freilandfläche, die zur Aussaat zu Verfügung steht, wird der Einsatz von 0,6 g Samen empfohlen, was ungefähr 2.000 - 3.500 Körnern entspricht.

Eine möglichst frühe Aussaat soll beim Anwuchs der Samen Spätfrostschäden vermeiden. Außerdem wird dadurch die Wachstumszeit der Sämlinge verlängert, indirekt die Qualität verbessert und die Gefahr von Sonnenbrand verringert, da mehr Zeit zur Adaption der Freilandstrahlung besteht. Eine Abdeckung mit Plastikplanen erhöht die Temperatur und verhindert die Verbreitung von Krankheitserregern. (Zhu et al., 1986)

\subsection{Anzucht in Pflanzcontainern}

Eine Anzucht von Sämlingen in Pflanzcontainern hat eine ganze Reihe von Vorteilen. Durch das Abdecken der Container mit Folien oder das Aufstellen in Gewächshäusern kann die Aussaat im Durchschnitt 4-8 Wochen früher erfolgen als vergleichsweise im Freiland. Dieser Wuchsvorsprung führt zu deutlich stabileren und besser wachsenden Pflanzen, die auch leichter bezüglich auftretender Krankheiten kontrolliert werden können. (Zhu et al., 1986)

Abhängig von der Größe der Container und dem Anwuchserfolg können die Pflanzen, bis zur Anpflanzung in diesen Container verbleiben oder noch einmal in eine Freifläche umgesetzt werden. Dabei sollte aber die Bodentemperatur beachtet werden, da diese großen Einfluss auf die Geschwindigkeit des Pflanzwachstums nimmt. Zhu et al. (1986) empfehlen die Verschulung der Pflanzen im Freiland erst ab einer durchschnittlichen Tagestemperatur von $16-18{ }^{\circ} \mathrm{C}$ und einer Bodentemperatur von $18{ }^{\circ} \mathrm{C}$ in $5-10 \mathrm{~cm}$ Bodentiefe.

Zur Vermeidung von Krankheiten befürworten die Autoren die Ausbringung von der Bordeaux-Mixtur (1.1.7 Biotische Risiken) in regelmäßigen Abständen von 10 - 15 Tagen. Auch sollten mechanische Beschädigungen an den Pflanzen unbedingt vermieden werden bzw. beschädigte Pflanzen aussortiert werden. 


\subsubsection{Setzlinge}

Da die Paulownia zur Ausbildung einer pseudo-dichotomen Astverzweigung neigt, ist bei der Anzucht von Setzlingen auf eine Auswahl von besonders stammwüchsigen Exemplaren zu achten, weil dieser erste Stammabschnitt in den Folgejahren den Großteil des Holz- und somit Wertzuwachses erbringt.

\subsection{Anzucht von Setzlingen aus Samen}

Unter normalen Wuchsbedingungen sind die folgenden vier Kriterien, laut Zhu et al. (1986) entscheidend zur Auswahl von Setzlingen im ersten Wuchsjahr:

- Der Stamm sollte geradschaftig, stabil und lignifiziert sein.

- Ein gleichmäßiges, möglichst großes Wurzelsystem muss ausgebildet sein.

- Es dürfen keine Krankheitsanzeichen oder mechanische Beschädigungen vorhanden sein.

- Das Verhältnis von Pflanzenhöhe zu Wurzelhalsdurchmesser (H/DVerhältnis) sollte im Bereich zwischen 60 - 70 liegen (bei P.fortunei bei ca. $60)$.

Die dabei von den Autoren geforderte mittlere Pflanzenhöhe bei ca. $4 \mathrm{~m}$ und der Mittendurchmesser von ca. $6 \mathrm{~cm}$, gelten ebenfalls als ungefähre Richtwerte.

Ergänzt werden diese Empfehlungen noch durch einen Hinweis auf die erschwerten Wuchsbedingungen in Bergregionen.

Auf Grund der dort schwierigen Transportwege bietet es sich an eher kleinere Pflanzen zu verwenden und diese nach dem ersten Jahr des Anwuchses bodentief abzuschneiden, auf dass sich im Folgejahr aus dem Wurzelstock ein möglichst langer, geradschaftiger Stamm herausbilden kann.

\subsubsection{Vegetative Vermehrung}

\subsubsection{Wurzelstecklinge}

Eine der in China am häufigsten eingesetzten Methoden zur Vermehrung von Paulownia ist die Verbreitung über Wurzelstecklinge, die zuvor von $1-2$ jährigen Pflanzen bzw. von ausgewachsenen Bäumen entnommen wurden.

Laut den Autoren Zhu et al. (1986) kann ein einjähriger Setzling zwischen 25 - 30 Wurzelstecklinge erbringen. Somit können in einer Baumschule, die ca. 6000 Setzlinge pro Hektar anpflanzt, ungefähr $150.000-180.000$ Wurzelstecklinge pro Jahr und Hektar gewonnen werden.

Diese Wurzelstecklinge sind leicht zu gewinnen, haben eine hohe Überlebens- bzw. Anwuchsrate und zeigen ein hohes konstantes Pflanzenwachstum.

\subsection{Gewinnung von Wurzelstecklingen}

Am leichtesten lassen sich Stecklinge in der Vegetationsruhe von den Wurzeln von einbis zweijährigen Pflanzen gewinnen. Die Wurzeln sollten ca. $1-4 \mathrm{~cm}$ dick sein und anhand dieser Durchmesser auch vorsortiert werden und generell auf eine Länge von $15-18 \mathrm{~cm}$ geschnitten werden. 
Je dicker der Wurzelsteckling ist, umso höher sind die Viabilität (Lebensfähigkeit) und das spätere Pflanzenwachstum. Um gutes Anwuchsverhalten zu ermöglichen sollten die Stecklinge nicht kopfstehend verpflanzt werden.

Bevor die Wurzelteile unter feuchten und kühlen Bedingungen (Durchschnittstemperatur im Bereich zwischen $0-10^{\circ} \mathrm{C}$ ) über den Winter hinweg eingelagert werden, sollten sie ausgebreitet ein bis zwei Tage trocknen.

Um ein natürliches Aufbrechen der Wurzelepidermis und die Ausbildung von neuen Wurzelspitzen zu ermöglichen, sollte ungefähr eine Woche vor dem Verpflanzen die Umgebungstemperatur auf $12-18{ }^{\circ} \mathrm{C}$ erhöht werden.

$\mathrm{Ab}$ einer Bodentemperatur von mindestens $5^{\circ} \mathrm{C}$ können die Stecklinge ausgebracht werden, wobei eine höhere Temperatur deutlich schnelleres Wachstum zeigt. (Zhu et al., 1986)

\subsection{Vorbereitung des Pflanzbeets}

Das Pflanzbeet sollte geschützt von starken Winden sein, dabei aber volle Sonneneinstrahlung ermöglichen. Der Boden sollte möglichst fruchtbar, gut durchlüftet, tiefgründig und drainiert sein und das Grundwasser frühestens ab einer Tiefe von 1,5 m ansetzen.

Das vorbereitende Pflügen des Bodens in einer Mindesttiefe von $40 \mathrm{~cm}$ ermöglicht eine gute Belüftung und Ausbildung des Wurzelsystems.

Um die Ansteckungsgefahr mit Krankheiten zu vermeiden sollte das Pflanzbeet möglichst weit von Mutterbäumen entfernt liegen und die Fläche auch nur einmal zur Vermehrung von Paulownia eingesetzt werden. (Zhu et al., 1986)

In China werden laut Zhu et al. (1986) zwei Formen von Pflanzbeeten bevorzugt, wobei die häufigste Variante das klassische Flachbeet ist.

Bei der zweiten Form handelt es sich um ein langgezogenes ca. $20 \mathrm{~cm}$ hohes und $1 \mathrm{~m}$ breites Hochbeet. Es bietet den Vorteil, dass sich der Boden schneller erwärmt und der evtl. künstlich eingebrachter Dünger auf diese kleinere Fläche konzentriert werden kann. Versuche haben gezeigt, dass die Pflanzen unter sonst gleichen Bedingungen im Durchschnitt $7 \%$ höher und am Wurzelhalsdurchmesser um bis zu $21 \%$ dicker wurden. Nachteilig sind aber die erhöhte Windanfälligkeit, das höhere Risiko zur Bindung von Säuren (Alkalinität) in dem Boden und ein evtl. eintretender erhöhter Wasserstress für die Pflanzen durch die Sonneneinstrahlung.

\subsection{Anzucht von Setzlingen aus Wurzelstecklingen}

Um eine möglichst hohe Anwuchsrate zu erzielen, empfehlen die Autoren Zhu et al. (1986) die Wurzelstecklinge im Frühjahr erst ab einer durchschnittlichen Bodentemperatur von $4-8{ }^{\circ} \mathrm{C}$ anzupflanzen (voraussichtlich Ende Mai). Die Stecklinge sollten, mit dem dickeren Ende nach oben, bis ca. $1 \mathrm{~cm}$ unter die Oberfläche senkrecht in die Erde gesteckt werden. Anschließend sollte ein wenig Erde in Höhe von $5-7 \mathrm{~cm}$ darüber aufgeschüttet werden, was die Bodentemperatur erhöht und Feuchtigkeit begünstigt. Die großflächige Abdeckung mit einer Folie erzielt bzw. verstärkt diese erwünschten Effekte. 
Eine zu hohe Pflanzendichte wirkt sich negativ auf die Überlebensrate aus, da die Pflanzen in Konkurrenz um Wasser und Licht stehen und sich bspw. durch Wind gegenseitig mechanische Beschädigungen zufügen können. Pflanzverbände von $1 \times 0,8$ m (12.600 Pflanzen pro ha) bzw. 1 x 1 m (10.000 Pflanzen pro ha) haben sich bei einem zu erwartenden Höhenzuwachs von $5-6 \mathrm{~m}$ in der ersten Vegetationsperiode als zweckmäßig herausgestellt. (Zhu et al., 1986)

Beim Ausgraben der Setzlinge aus dem Baumschulboden und vor dem Weitertransport zur Aufforstungsfläche, kann bewusst ein Teil der Wurzeln im Boden verbleiben und im kommenden Frühjahr wieder für neue Setzlinge sorgen. Diese Wurzelstücke sollten dabei mindestens $1,5 \mathrm{~cm}$ Durchmesser haben und möglichst gleichmäßig über die Fläche verteilt sein. Diese kosten- und arbeitssparende Methode ist in China alltäglich, hat aber den Nachteil, dass sich Krankheiten schneller verbreiten können. (Zhu et al., 1986)

\subsubsection{Gewinnung von Ablegern}

Laut Zhu et al. (1986) ist die Gewinnung von Ablegern sowohl aus Stammabschnitten von Setzlingen als auch aus Aststücken von mehrjährigen Pflanzen möglich. Diese Form der vegetativen Vermehrung wird aber im Vergleich zu Wurzelstecklingen als bei weitem aufwendiger und im Anwuchsverhalten als weniger erfolgreich eingestuft und wird daher nur in Ausnahmen, in denen bspw. nicht genügend gutes Wurzelmaterial zu Verfügung steht, empfohlen.

Bei der Auswahl von Stamm- bzw. Aststücken ist auf ausreichende Dicke, Länge und dem Vorhandensein von möglichst vielen Nodien zu achten. An den Stammabschnitten bzw. den Aststücken können sich dann sehr leicht Wurzelknospen bilden, die sich zu Adventiv-Wurzeln weiterentwickeln.

Die Anwuchsrate bei Aststücken ist aber vergleichsweise gering und der Aufwand daher nur zur schnellen Gewinnung und Selektion von besonders wuchskräftigen Klonen zu empfehlen. (Zhu et al., 1986)

\subsubsection{Pflanzung}

Die Paulownia verfügt über viele Merkmale einer Pionierbaumart und kann daher auf Extremstandorten wachsen. Am besten gedeiht sie aber auf fruchtbaren, tiefgründigen, lockeren, sandigen Lehmböden bzw. auf puren Lehmböden, wenn das Grundwasser tiefer als 1,5 $\mathrm{m}$ ansetzt. Die Anpassungs- und Wachstumsfähigkeit variiert zwischen den Arten, aber generell findet auf sehr tonigen, trockenen oder armen Böden nur ein stark vermindertes Wachstum statt. Ebenso sind winterliche Temperaturen unter $-20{ }^{\circ} \mathrm{C}$, hochanstehendes Grundwasser und sehr windexponierte Standorte aufgrund der großen Blätter nachteilig. (Zhu et al., 1986) 


\subsubsection{Auswahl des Pflanzverbandes}

\subsection{Traditioneller Pflanzverband}

Rein traditionell erfolgt in China der Anbau zur Eigenversorgung der Bevölkerung mit Holz an den „Vier Seiten“. Diese sind die Umgebung vom eigenen Haus, die Flächen um das Dorf und die Seitenstreifen entlang von Straßen und Flussläufen, die meist aus tiefgründigen, fruchtbaren und leicht zu bewirtschaftenden Böden bestehen. Laut Zhu et al. (1986) sollten die Bäume in der Nähe von Häusern und Dörfern in Streifen bzw. als Gruppen mit einem Pflanzverband von $5 \times 5 \mathrm{~m}$ bzw. $5 \times 6 \mathrm{~m}$ gepflanzt werden. In der fünften bzw. sechsten Vegetationsperiode erfolgt dann eine Durchforstung, um die Baumanzahl zu halbieren und somit den Wuchsraum für die verbleibenden Bäume zu verdoppeln.

\subsection{Allgemeine Pflanzverbände}

Zur Anlage von Plantagen empfehlen Zhu et al. (1986) eine flexible Anpassung an die standörtlichen Gegebenheiten wobei sich Pflanzverbände mit 6 × 6 m (278 Pflanzen / ha), $5 \times 5$ m (400 Pflanzen / ha) und $5 \times 4$ m (500 Pflanzen / ha) bewährt haben. Generell sollten einzelbaumweise Durchforstungen bzw. die schematische Entnahme jeder zweiten Reihe im fünften bzw. sechsten Jahr nach der Pflanzung erfolgen. Dadurch vergrößern sich die Verbände auf $6 \times 12 \mathrm{~m}, 5 \times 10 \mathrm{~m}$ und $5 \times 8 \mathrm{~m}$ und der Wuchsraum pro Einzelbaum erhöht sich auf $72 \mathrm{~m}^{2}, 50 \mathrm{~m}^{2}$ und $40 \mathrm{~m}^{2}$. Abhängig von dem noch zu erwartenden Durchmesserzuwachs können weitere Durchforstungen erfolgen.

\subsubsection{Anpflanzung}

\subsection{Pflanzung von Jungpflanzen}

Eine Anpflanzung mit Pflanzen aus dem gleichen Jahr sollte im Herbst erfolgen, wenn das Höhenwachstum abgeschlossen ist, die Blätter aber noch nicht abgefallen sind. Nach Möglichkeit sollte zu diesem Zeitpunkt die Bodentemperatur noch hoch sein, auf dass sich neue Wurzelenden bilden können, die wiederum im kommenden Frühjahr die Überwindung des Pflanzschocks erleichtern.

Zhu et al. (1986) empfehlen die Pflanzen vorab nach Höhen zu sortieren. Generell sollten große Pflanzen nicht auf armen Böden und Standorte mit hoher Windanfälligkeit verpflanzt werden. Das Wurzelsystem sollte bei der Entnahme aus dem Pflanzbeet so gut wie möglich erhalten bleiben.

\subsection{Pflanzung von Wurzelstecklingen}

Wenn statt Pflanzen die Anpflanzung mittels Wurzelstecklingen erfolgt, so hat dies eine Reihe von Vorteilen wie bspw., dass auf die Vorab-Arbeitsleistung einer Baumschule verzichtet werden kann, dass somit ein Pflanzschock vermieden wird und die Pflanzen direkt am Ort ihrer Bestimmung von Beginn an gedeihen. Nachteilig sind aber die schwierigere Bewirtschaftung und die Ungleichmäßigkeit in der Höhenentwicklung. Daher empfiehlt es sich, die Pflanzen nach der ersten Vegetationsperiode wieder abzuschneiden, auf dass sie im zweiten Jahr verstärkt austreiben können. (Zhu et al., 1986) 
Diese Fähigkeit der Paulownia, nach dem Fällen des Baumes wieder auszutreiben, wird in China in der bevorzugten Bewirtschaftungsform der Kurzumtriebsplantage verwendet. Diese einfachste Form der indirekten Neuanpflanzung hat auf großer Fläche aber den Nachteil, dass sich schnell Krankheiten verbreiten können und empfiehlt sich daher nur für wenige Generationsfolgen. (Zhu et al., 1986)

\subsubsection{Wuchsverhalten von Jungpflanzen}

Um das Wuchsverhalten der Pflanzen zu fördern, empfehlen Zhu et al. (1986) folgende drei Aspekte besonders zu berücksichtigen:

- Da sowohl die Größe als auch die Anzahl der Blätter die Gesamtphotosyntheseleistung und somit direkt das Wachstum der Pflanze bestimmen, sollte nach Möglichkeit keines der Blätter beschädigt bzw. vor dem natürlichen Laubfall entfernt werden.

- Die regelmäßige Kontrolle der Jungpflanzen, um die Ausbreitung von Krankheiten wie bspw. der Anthraknose (1.1.7 Biotische Risiken) zu verhindern und den Befall mit Raupen und blattfressenden Insekten zu verringern.

- Staunässe bzw. dauerhaft vollgesättigte Böden führen zu einem Absterben der Pflanzen binnen drei bis vier Tagen und sollten daher unbedingt vermieden bzw. mittels Aushub von Abflussgräben abgewendet werden.

\subsubsection{Wachstumsphasen von Jungpflanzen}

Das Wuchsverhalten aller Jungpflanzen ist in der ersten Vegetationsperiode ähnlich und lässt sich grob in die drei Phasen: Anwuchs, Wachstum und Reife unterteilen.

\subsection{Anwuchsphase}

In der Anwuchsphase investiert die Pflanze ihre Energie in die Etablierung des Wurzelsystems und die austreibenden Triebe wachsen nur sehr langsam (Longbrake, 2001). Sowohl bei der Anpflanzung durch Samen und erst recht bei der durch Wurzelstecklinge, bilden sich immer mehrere Knospen heraus. Um die Wuchsernergie der Pflanze auf einen kräftigen Haupttrieb zu lenken, sollten die schwächeren Triebe ab einer Höhe von $5 \mathrm{~cm}$ abgekniffen werden. Die Fähigkeit Stockausschläge zu bilden, konnte bei den Sämlingen nach ca. 4 Wochen seit der Keimung festgestellt werden (Longbrake, 2001). Sobald die Pflanzen Höhen von $10 \mathrm{~cm}$ erreicht haben, kann eine zusätzliche Bewässerung erfolgen, ohne dass mit größeren Schäden an den noch fragilen Pflanzenteilen zu rechnen ist. (Zhu et al., 1986)

\subsection{Wachstumsphase}

Die Hauptwachstumsphase erfolgt ungefähr in den Monaten von Ende Juni bis Mitte August, wenn die Tagestemperaturen am höchsten sind. Diese Phase ist entscheidend für die Qualität und Quantität der Pflanzen, da sich in dieser Zeit ca. 75 - $80 \%$ des Höhenzuwachses der gesamten Vegetationsperiode herausbilden. 
Das Wurzelsystem hat sich etabliert und wächst auch weiterhin zu, aber die Pflanze lenkt ihre Energie vermehrt in die Erhöhung des Blattfächenindexes. Sowohl der durchschnittliche Höhen- als auch der Durchmesserzuwachs pro Tag erreichen ihr Maximum. (Zhu et al., 1986)

Bei besonders schnellgewachsenen Pflanzen besteht die Gefahr des Umfallens bzw. Krümmens und daher raten Zhu et al. (1986) am Stammfuß zusätzliche Erde anzuschütten um die Herausbildung von Seitenwurzeln zu fördern. Auch der regelmäßige Einsatz von schnellwirkendem Dünger und zusätzlicher Bewässerung, um das Wachstum zu fördern, wird empfohlen.

Da beispielsweise P.fortunei (ähnlich wie P.catalpifolia) dazu neigt schon im ersten Jahr Seitenäste zu bilden, sollten diese zur Pflege eines möglichst langschaftigen, astfreien Stammes entfernt werden.

\subsection{Reifephase}

Die Reifephase der Pflanzen beginnt ungefähr Ende August, wenn die durchschnittliche Tagestemperatur unter $20^{\circ} \mathrm{C}$ fällt. Diese Phase wird davon gekennzeichnet, dass sich das Höhenwachstum sehr schnell verringert und Mitte September ganz einstellt. Im Vergleich dazu verringert sich das Dickenwachstum nur langsam und endet erst spät ca. Mitte Oktober mit dem Laubabwurf.

In dieser Phase sollte keine zusätzliche Bewässerung bzw. Düngung mehr erfolgen, da dies kontraproduktiv gegenüber der nun einsetzenden natürlichen Lignifizierung der Triebe wäre. Nicht mehr rechtzeitig verholzte Triebe sterben in der Vegetationsruhe, abhängig von der Tiefe und Dauer der winterlichen Temperaturen, ab und können im kommenden Frühjahr an der Stammbasis erneut austreiben. (Zhu et al., 1986)

\subsubsection{Regenerierung von minderwüchsigen Jungpflanzen}

Wenn sich einzelne Jungpflanzen im Kontrast zu anderen gleichaltrigen Exemplaren nur unzureichend in der Baumschule entwickelt haben, dann empfehlen Zhu et al. (1986) das saubere Zurückschneiden des Haupttriebes bis zum Wurzelhals. Dies sollte in der Vegetationsruhe im Winter bzw. Frühjahr noch vor dem Austrieb erfolgen.

Da sich bereits in der ersten Vegetationsperiode das Wurzelsystem weitestgehend etabliert hat (Longbrake, 2001), ist mit einem stärkeren und frühen (bis zu einen Monat) Austrieb der Pflanze zu rechnen. Die Wachstumsrate ist ähnlich frischgepflanzter Wurzelstecklinge, mit dem Unterschied, dass die Verholzung zum Ende der Vegetationszeit ca. 14 Tage früher einsetzt, was weniger winterliche Schäden zur Folge hat. (Zhu et al., 1986) 


\subsubsection{Pflege und Förderung}

\subsubsection{Allgemeine Pflege und Bewirtschaftung}

Eine intensive Pflege der Jungpflanzen ist in den ersten drei bis vier Jahren von großer Bedeutung, um ein optimales Wachstum mit möglichst geradem Stammverlauf zu ermöglichen.

\subsection{Vermeidung von Schäden}

Da die Pflanzen vor allem in den ersten zwei Jahren noch sehr empfindlich sind, können starke Winde und plötzlich auftretender Platzregen das Blattwerk schädigen bzw. im schlimmsten Fall das Umknicken der Stämme zur Folge haben. In dem Falle kann es ratsam sein am Ende der Vegetationsperiode die Pflanze bis auf Höhe ihres Wurzelansatzes zurückzuschneiden und einen neuen starken Austrieb im kommenden Frühjahr herauszupflegen.

Die Rinde ist anfänglich noch sehr dünn und Verletzungen überwallen nur schlecht und langsam und haben definitiv Einfluss auf die spätere Holzqualität. Daher müssen jegliche Formen von Beschädigungen vermieden werden, indem bspw. die Fläche gezäunt wird, um Verbiss bzw. Schälung auszuschließen. Das Risiko von Frostschäden und Sonnenbrand kann durch das Einpacken des Stammes mit Grasbündeln bzw. durch einen Kalkanstrich der Rinde, minimiert werden. (Zhu et al., 1986)

\subsection{Bewässerung}

Die Paulownia kann ohne Probleme größere Trockenzeiten überstehen. Da sie aber schon nach kurzer Zeit mit dem Abwurf der Blätter reagiert, verringert sich folglich der Holzzuwachs. Zur Vermeidung solcher Trockenphasen empfiehlt Zhu et al. (1986) eine regelmäßige, künstliche Bewässerung im Wochentakt. Dabei darf es aber auf keinem Fall zu Staunässe kommen, da diese bereits schon nach 3-4 Tagen zum Absterben der Jungpflanzen führen kann.

\subsubsection{3 Ästung}

Abhängig vom Wachstumsverlauf der Jungbäume kann mit der Ästung, im dritten oder vierten Jahr nach der Pflanzung, zu Beginn der Winterruhe begonnen werden.

Beispielsweise hat zu dem Zeitpunkt die P.elongata, die vergleichsweise das schnellste Wachstum aller Arten zeigt, bereits mit der verstärkten Ausbildung der lateralen Krone begonnen.

Zur Erziehung eines möglichst astfreien Stammabschnittes, sollten alle Äste vom Stamm bis zum Beginn der Hauptkrone entfernt werden. Um den Kronenverlust bei der ersten Ästung gering zu halten und die Gefahr von Stammfäule zu verringern, empfehlen Zhu et al. (1986) anfänglich nur die Entfernung der schwächeren und den Erhalt der dickeren Seitenäste. 


\subsection{Durchforstung}

Der Wachstumsverlauf bestimmt direkt und die Dichte des Pflanzverbandes indirekt den Zeitpunkt der ersten Durchforstung. Sobald sich die Äste der Bäume untereinander zu berühren beginnen, ist dies nachteilig für das Wachstum, da sie sich dann auch gegenseitig beschatten. Dies ist der späteste Zeitpunkt für die erste Durchforstung, die bei Kurzumtriebsplantagen schematisch in reihenweiser Form oder einzelbaumweise erfolgen kann. Zur Förderung der besonders langschaftigen, qualitativ hochwertigen und stabilen Bäume sollte im Alter von 5 - 6 Jahren eine Durchforstung durchgeführt werden. (Zhu et al., 1986)

\subsubsection{Förderung des Stammwachstums}

\subsection{Verzweigungstypen}

Wenn ein Teil des Terminaltriebes im Winter zurückfriert dann treibt die Paulownia mittels Seitenknospen im kommenden Frühjahr erneut aus. Es bildet sich dabei eine pseudo-dichotome Astverzweigung, deren Stärke und Verzweigung sich zwischen den Arten unterscheidet.

Zhu et al. (1986) charakterisieren drei Wachstumstypen, die unterschiedlich behandelt werden sollten, um ein optimales Durchmesserwachstum zu ermöglichen:

- Bei der P.fortunei (ebenso P.albiphloea, P.taiwaniana, P.catalpifolia) bildet sich an der Spitze des Terminaltriebes ein Astpaar aus, von dem sich der stärkere Ast zum neuen Terminaltrieb weiterentwickelt, und der zweite, schwächere Ast schräg bzw. horizontal weiterwächst. Dadurch entsteht auf natürliche Weise ein gerader, starker Stamm und es bedarf keiner weiteren Pflegemaßnahmen.

- Die P.elongata (ebenso P.fargesii, P.australis) hingegen zeigt eine unregelmäßige Verzweigung der Äste die unterschiedlich stark weiterwachsen. Ab dem dritten bzw. vierten Jahr nach der Pflanzung sollte der stärkste und geradeste Hauptast gefördert werden, indem die Seitenverzweigung entfernt wird.

- Die P.tomentosa (ebenso P.kawakamii, P.tomentosa var. tsinlingensis) entwickelt eine Verzweigung, bei der sich die Äste meist gegenseitig in ihrer Entwicklung hemmen. Der Pflegeaufwand mit dem Zurückschneiden von unerwünschten Ästen ist hier am größten.

\subsection{Pflegeschnitt am Wurzelhalsansatz}

Wenn das Wachstum der Pflanzen in der ersten Vegetationsperiode nach der Pflanzung nicht überzeugt bzw. Schädigungen aufgetreten sind, dann besteht die Möglichkeit sie bis zum Wurzelhals komplett zurückzuschneiden.

Das bringt den Vorteil, dass die Pflanzen vorab ein Wurzelgeflecht etablieren konnten und dann in der zweiten Vegetationsperiode mit kräftigem und geradem Terminaltrieb neuaustreiben können. Dabei eventuell auftretende kleinere Nebentriebe sollten zeitnah, beim Beginn der Vegetationsperiode oder im Herbst des gleichen Jahres, entfernt werden. (Zhu et al., 1986) 


\subsection{Pflegeschnitte an der Baumspitze}

Um einen möglichst geraden und langen Stammabschnitt zu erzeugen, empfiehlt es sich in der zweiten bzw. dritten Vegetationsperiode Pflegeschnitte an der Baumspitze vorzunehmen.

Eine Möglichkeit besteht darin die komplette Krone unterhalb des Hauptastes abzusägen. Von den obersten Knospen wird sich in der nächsten Vegetationsperiode ein Starkast herausbilden, der durch weitere Pflegeschnitte gefördert werden sollte.

Alternativ dazu können auch nur alle obersten Äste entfernt werden und nur der stärkste schon vorhandene Ast verbleibt und bildet die Erweiterung des Stammes in der nächsten Vegetationsperiode. Die restliche Krone unterhalb dieses Abschnittes verbleibt ebenfalls am Baum und unterstützt mittels ihrer Blattfläche das Wachstum im nächsten Jahr. (Zhu et al., 1986)

Generell ist eine solche Stammerweiterung durch die Selektion von Knospen einfach in der Umsetzung und hat in einem solch frühen Stadium der Baumentwicklung keinen negativen Einfluss auf die Holzqualität. Die geförderte Knospe sollte aber unbedingt aus einer der obersten drei Paare bestehen. Auch sollten alle nach oben strebenden, schwächeren Seitenäste komplett entfernt werden und die zur Unterstützung verbleibenden sich horizontal entwickelnden Seitenäste, wenn sie ca. $20-30 \mathrm{~cm}$ lang sind, ebenfalls an ihrer Spitze beschnitten und im Jahr darauf komplett entfernt werden. Die entstehenden Astwunden überwallen in der Regel binnen zwei bis drei Jahren und sollten zur Vermeidung von Austrocknungserscheinungen und dem Eindringen von Pilzsporen mit nassem Lehm bestrichen werden. (Zhu et al., 1986)

\subsection{Kronenschnitte}

Wenn die Pflanzen im dritten bzw. vierten Jahr noch immer keinen ausreichend großen Stamm herausgebildet haben und auch keine geeigneten Äste für einen Pflegeschnitt an der Baumspitze vorhanden sind, dann ist eine Ästung der gesamten Krone möglich und evtl. nötig.

Am Hauptstamm sollte eine obere, starke, noch schlafende Knospe, im Frühling ungefähr zwei Wochen vor Beginn des Austriebes, ausgewählt werden. Ein Schnitt sollte den Stamm zwei, drei Zentimeter oberhalb dieser Knospe zu ca. zwei Drittel durchtrennen, was die Versorgung der Knospe fördert und ihr Wachstum beschleunigt. Die noch lebenden Teile der Krone können in der kommenden Vegetationsruhe entfernt werden. Kleinere Äste unterhalb des neuen Terminaltriebes, die sich aus der geförderten Knospe entwickelt haben, sollten ein Jahr länger am Baum verbleiben, um die komplette Entfernung der Kronenfläche über zwei Vegetationsperioden hinweg zu strecken.

Generell sollte bei einer so starken Beschneidung der Krone Rücksicht, sowohl auf die individuelle Entwicklung des Baumes, als auch auf die Standortbedingungen wie z.B. Windanfälligkeit genommen werden, um einen negativen Einfluss auf das Gesamtwachstum zeitlich so gering wie möglich zu halten. (Zhu et al., 1986) 


\subsection{Das Holz der Paulownia}

\subsubsection{Holzeigenschaften}

Eine tabellarische Auflistung der Holzeigenschaften der P.-Arten: P.elongata, P.fortunei, P.tomentosa, P.tomentosa var. tsinlingensis, P.catalpifolia und P.farbesii übernommen und verändert nach Z. H. Zhu et al. (1986) findet sich im Anhang zu dem Kapitel I (Tab. 12).

\subsubsection{Holzstruktur}

\subsection{Makroskopisch}

Die Beschaffenheit des Holzes schwankt von fein bis grob und die Farbe variiert von leicht gelblich bis rötlich. Es ist geruchlos, leicht und weich und nachdem es gehobelt wurde erscheint es glänzend. Das Holz lässt sich leicht hobeln, sägen oder mit Schnitzwerkzeugen bearbeiten, ohne dass es splittert. Das Splintholz besteht meist aus ein bis zwei Jahrringen und der Übergang zum Kernholz verläuft unscharf.

Das Kernholz kann in Abhängigkeit von der Geschwindigkeit des Durchmesserwachstums aus weiten Jahrringen bestehen, die sich nicht immer klar voneinander abtrennen lassen. Die Einteilung schwankt zwischen Semiring- und Ringporern. Sowohl die Holzstrahlen als auch die Holzporen sind mit dem Auge nicht erkennbar. (Zhu et al., 1986)

\subsection{Mikroskopisch}

Die Durchmesser der Leitbahnen zeigen Unterschiede beim Früh- zum Spätholz im Verhältnis 3 zu 5 und in Ausnahmen sogar 1 zu 10. Sie sind rund oder elliptisch geformt und finden sich eher einzelstehend im Gewebe. Die Verthyllung findet im Frühholz stärker statt als im Spätholz.

Die Holzstrahlen sind meistens homogen und nur gelegentlich heterogen, was bspw. bei P.fortunei häufig zu finden ist. Sie bestehen meist aus 1-50 Zellen in der Höhe und aus 1 - 8 Zellen in der Breite.

Das Holz-Parenchym ist meist in Bündelform anzutreffen und stark ausgebildet. Es verläuft parallel zu den Tracheen in ringartiger Ausprägung. Um die Leitbahnen des Spätholzes ist es am stärksten zu finden.

Die Holzfasern sind von ihrer Größe her sehr gleichmäßig und die Zellwand ist ca. $3 \mu \mathrm{m}$ dünn und ungefähr $550-1.700$ mm lang. (Zhu et al., 1986)

\subsubsection{Holzcharakter}

\subsection{Gewicht}

P.-Holz zeichnet sich durch sehr geringes Gewicht aus, wobei P.fargesii und P.elongata das leichteste Holz, bezogen auf die Holzmasse, ausbilden.

Die Dichte variiert zwischen den Arten und hängt auch stark von den Standortseigenschaften ab, liegt aber bei luftgetrocknetem Holz (Feuchtegehalt ca. $15 \%$ ) bei ca. $0,26-0,33 \mathrm{~g} / \mathrm{cm}^{3}$. 
Das sehr biegsame Holz hat nur geringe Festigkeit und kann daher bspw. nicht zum Hausbau als tragendes Element verwendet werden. Die Relation von Festigkeit zu Gewicht ist jedoch vergleichsweise hoch. Eine Verwendung bietet sich daher eher in Anwendungsfeldern für weiches aber relativ dazu festes Holz an. (Zhu et al., 1986)

\subsection{Holzfeuchte und Holzeigenschaften}

Die Holzfeuchte ist vergleichbar mit anderen häufig verwendeten Baumarten. Zhu et al. (1986) berichten davon, dass Produkte aus diesem Holz nur wenig anfällig für hohe Luftfeuchtigkeit und Insekten aller Art sind, denn das Holz kann binnen 25 Tagen bei normaler Raumtemperatur auf ca. $10 \%$ Holzfeuchte abtrocknen.

Produkte aus Paulownia-Holz reißen, verformen und verdrehen sich nur sehr wenig, was einer der größten Vorteile ist. Der Holzschrumpfungs-Koeffizient liegt im Durchschnitt bei ca. 0,27-0,37\%.

Die Wärme-Leitfähigkeit ist sehr gering und liegt bei ca. $0,063-0,086 \mathrm{Kcal} \mathrm{m}^{-1} \mathrm{hr}^{-1}{ }^{\circ} \mathrm{C}^{-1}$. Somit ist das Holz sehr gut für Isolierungen bspw. im Hausbau geeignet. (Zhu et al., 1986)

\subsection{Resistenz gegenüber Fäule}

Die Autoren Zhu et al. (1986) berichten dass noch keine ausreichenden wissenschaftlichen Untersuchungen zur Haltbarkeit des Holzes gegenüber Fäuleerregern in China vorliegen. Jedoch gibt es eine Reihe von Beobachtungen nach der auch eine mehrjährige Fäule auf dem Holz nur oberflächlich auftritt. Beispielsweise bei einem Versuch in Hong Ya, bei dem zum Vergleich eine Vielzahl von Bäumen verschiedener Arten gefällt und das Holz im Wald zurückgelassen wurde, zeigte sich nach ca. 15 - 16 Jahren, dass die Fäule nur ca. $1 \mathrm{~cm}$ in das Holz von Paulownia-Stämmen vorgedrungen war. Auch gibt es viele Berichte darüber, dass Särge aus Paulownia-Holz besonders haltbar sind und auch nach 30 Jahren nur geringe Anzeichen von Zersetzung zeigen (siehe auch 1.1.1.1.1 Historische Erwähnungen). (Zhu et al., 1986)

\subsubsection{Holzverwendung}

\subsubsection{Nutzung des Holzes}

\subsection{Baumaterial}

Die Festigkeit des Holzes ist nur gering und daher schließen sich eine Vielzahl von Verwendungsmöglichkeiten aus. Trotz dessen wird Paulownia-Holz, weil es leicht und formstabil ist, beim traditionellen Häuserbau bspw. für Dachbalken und Dachpfetten (waagerechter Träger in der Dachkonstruktion) bevorzugt verwendet. Auch der moderne Einsatz in Form von Spanplatten ist bereits erforscht (Michaelis, 2013). Weitere Anwendungsmöglichkeiten sind z.B. der Bau von Türen, Fensterrahmen, Raumteilern und zur Verkleidung von Zimmerdecken.

In den ländlichen Regionen Chinas werden ausgehöhlte Stämme für Wasserleitungen bzw. als Dachrinnen genutzt. (Hecker and Weisgerber, 2014; Zhu et al., 1986) 


\subsection{Möbelbau}

Beim Möbelbau wird das Holz für Tische und Stühle genutzt, da es nur wenig von Insekten angegriffen wird und durch seine Formstabilität sogar das nahezu luftdichte Verschließen von bspw. Schränken, Etuis und Kästen aller Art ermöglicht.

Langsam gewachsenes und somit feingemasertes Holz wird bevorzugt für den Bau von Öl-, Wein- und Bierfässern, Tee- und Fruchtboxen verwendet. Aber auch Rettungsinseln, Schlitten und Surfbretter werden daraus hergestellt.

Eine spezielle Anwendung der guten Isolierungseigenschaften und geringen Wärmeleitfähigkeit des Holzes betrifft den Bau von Bienenstöcken (Beute). (Hecker and Weisgerber, 2014; Zhu et al., 1986)

\subsection{Kunsthandwerk}

Im Kunsthandwerk gibt es eine Vielzahl von Anwendungen wie bspw. als geschnitzte Blumenvasen, für Buddha-Figürchen, Holzfische, usw. wobei die Rinde zum Färben genutzt wird.

In alter chinesischer Tradition werden auch heute noch aus der Holzkohle Malstifte hergestellt oder diese für Schwarzpulver zerstoßen, um dann in Feuerwerkskörpern zur Anwendung zu kommen. (Zhu et al., 1986)

Kays (1996) weist daraufhin, dass Japaner das Holz bevorzugt für Musikinstrumente, Schnitzarbeiten, Schüsseln, Löffel, Spazierstöcke, Paddel, Möbel und ganz besonders für die Mitgift (Aussteuer-Truhe) nutzen. Wohingegen Amerikaner das Holz bevorzugt für Schnitzblöcke, für Holzpfeifen zum Anlocken von Truthähnen, Gitarren, Särge bzw. Truhen und Skier verwenden.

\subsection{Nutzung der Holzfasern}

Da das Holz im Vergleich zu anderen Gehölzen ca. 40 \% leichter pro Volumeneinheit ist, wird es noch heute zum Bau von Booten und früher auch zum Flugzeugbau verwendet. (Hecker and Weisgerber, 2014; Zhu et al., 1986)

Bei einer Nutzung der Holzwolle als Füllmaterial für Transportboxen kann so indirekt das Gesamtgewicht der Boxen erhöht werden. Sehr häufig wird die Holzstreu auch für Kuhställe oder für Geflügelfarmen eingesetzt. In neuerer Zeit findet eher eine Verwendung der Holzfasern zur Herstellung von Zellstoff und somit zur Papiererzeugung statt.

Aus dem weitestgehend astfreien Holz lassen sich nach der Zerspanung sehr gut Sperrholzplatten herstellen. Furniere können bis auf eine Dünne von ca. 0,25 mm gehobelt werden, ohne dass die Fasern reißen. Die vielseitige Weiterverwendung dieser Ausgangsmaterialen in anderen Produktgruppen erklärt warum Paulownia eine der wichtigsten Bäume in der chinesischen Holzindustrie ist. (Zhu et al., 1986)

\subsubsection{Sonstige Verwendung}

\subsection{Medizinprodukte}

Laut Zhu et al. (1986) haben pharmazeutische Experimente gezeigt dass Extrakte aus den Früchten der Paulownia in Form von Tabletten oder Injektionen sowohl Husten als auch Asthmaanfälle lindern können und zur Reduzierung von hohem Blutdruck verwendet werden. 
In der alten chinesischen Medizin wird dazu geraten die Blätter und Früchte in Wasser aufzulösen und diese Lösung täglich in die Kopfhaut zu massieren, was das Haarwachstum fördern soll und angeblich graues in schwarzes Haar zurückverwandelt. Die gleiche Lösung kann aber ebenso zur Behandlung von geschwollenen Füßen genutzt werden. (Zhu et al., 1986)

\subsection{Weitere Nutzungen}

Die Blüten und Blätter können aufgrund ihres vergleichsweise hohen Gehalts an pflanzlichem Fett, Zucker und Protein als Futter für Schweine, Schafe und Kaninchen genutzt werden. Auch die Einarbeitung der Blattstreu in den Ackerboden als zusätzlicher Dünger wird häufig praktiziert.

Traditionell werden in China die Musikinstrumente aus Paulowniaholz hergestellt. Die guten Resonanzeigenschaften des Holzes zeigen sich speziell bei Streichinstrumenten und deren Klangböden. (Hecker and Weisgerber, 2014; Zhu et al., 1986)

In der stark von Umweltverschmutzung geprägten Stadt Chengdou (Szechuan Provinz) wird die Paulownia bevorzugt, aufgrund ihrer großen haarigen Blätter, zur Luftreinigung angebaut. Beobachtungen haben gezeigt, dass die Baumart auch noch in verschmutzten Regionen wächst in der andere Arten das Wachstum einstellen bzw. absterben. (Zhu et al., 1986) 


\section{Kapitel II}

\section{Invasivität, Kurzumtrieb und Agroforst}

\subsection{Die Invasivitäts-Frage}

\subsubsection{Zur Einführung von gebietsfremden Arten}

\subsubsection{Historie und Begrifflichkeiten}

Das Jahr 1492, in welchem Christoph Kolumbus (ital. Cristoforo Colombo), ein italienischer Seefahrer in kastilischen Diensten, eine Insel der Bahamas erreichte, ist als die Entdeckung Amerikas in die Geschichtsbücher eingegangen. Dieses in vielerlei Hinsicht wichtige Datum markiert einen offiziellen Wendepunkt bei der Einteilung und somit indirekt bei der Bezeichnung von einheimischen und gebietsfremden Arten.

Alle Arten, die gebietsfremd sind und vor 1492 absichtlich oder unbeabsichtigt durch menschliche Aktivitäten neueingeführt wurden, bspw. durch die großräumigen Handelswege der Römer, werden als Archäobiota (Archäophyten = „Alt-Pflanzen“, Achäozoen = „Alt-Tiere“) bezeichnet. Wenn die Einführung von gebietsfremden Arten nach 1492

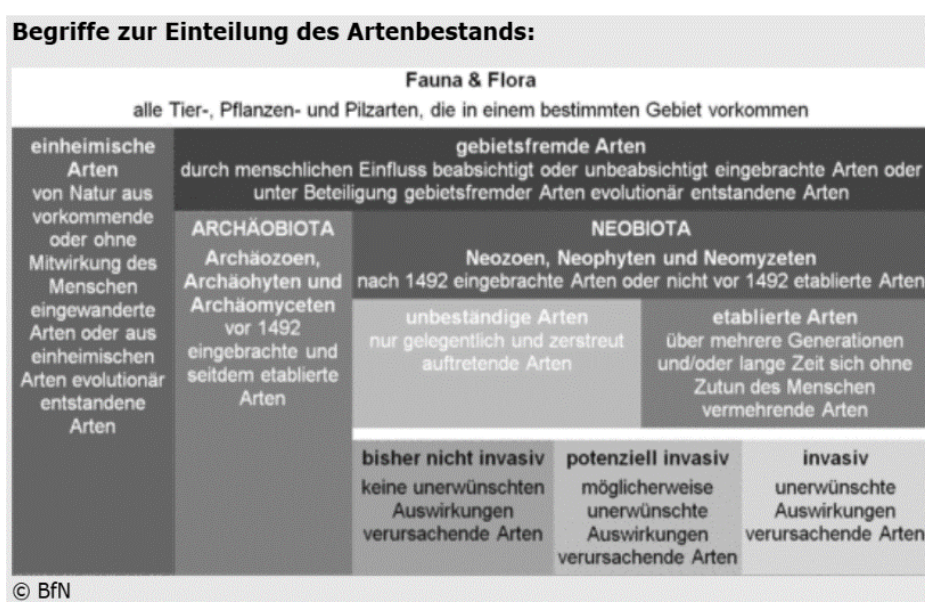

Abb. 7: Begriffe zur Einteilung des Artenbestands, verändert nach Neobiota (2020). erfolgte, so werden diese als Neobiota (Neophyten $=$ "NeuPflanzen“, Neozoen = „NeuTiere") bezeichnet. Als etabliert gelten diese Artenn wenn sie sich über mehrere Generationen hinweg ohne menschlichen Einfluss erhalten können. Diese Arten werden sprachlich von den einheimischen (Indigenen) abgegrenzt und als Agriophyten (Pflanzen) bzw. Agriozoen (Tiere) bezeichnet (Abb. 7). (Neobiota, 2020)

Wenn diese gebietsfremden Arten unerwünschte Auswirkungen auf einheimische Arten oder deren Lebensgemeinschaften bzw. Biotope haben, so werden sie seitens des Naturschutzes als invasiv bezeichnet (Neobiota, 2020).

Unter dem Artikel $\S 7$ Abs. 2 Nr. 9 BNatSchG wird der Begriff „invasive Art“ rechtlich bestimmt. Danach darf eine gebietsfremde Art als invasiv bezeichnet werden, insofern eine erhebliche Gefährdung der biologischen Vielfalt vorliegt. (BNatSchG, 2009)

Wissenschaftlich korrekt wird laut Kowarik (2010) unter einer Invasion ein Gesamtprozess verstanden, bei dem ein gebietsfremder Organismus ein neues Gebiet besiedelt. Dabei ist es unbedeutend ob dies mit unerwünschten Auswirkungen einhergeht. 
Die Tab. 3 gibt einen Überblick über die vom Bundesamt für Naturschutz ermittelte aktuelle Anzahl Neobiota ${ }^{13}$ in Deutschland und ihre naturschutzfachliche Invasivitätsbewertung ( ${ }^{1}$ nach BfN, ${ }^{2}$ nach Nehring et al., 2010, 2013, 2015 \& Rabitsch et al., 2013):

Tab. 3: Aktuelle Anzahl Neobiota in Deutschland, Stand 2015.

\begin{tabular}{|c|c|c|c|c|}
\hline \multirow[t]{2}{*}{ Gruppe } & \multirow[t]{2}{*}{ Status } & \multirow{2}{*}{$\begin{array}{l}\text { Anzahl wild lebender } \\
\text { Neobiota-Arten in } \\
\text { Deutschland }{ }^{1)}\end{array}$} & \multicolumn{2}{|c|}{ Naturschutzfachliche Invasivitätsbewertungen ${ }^{2)}$} \\
\hline & & & Invasive Arten & Potenziell invasive Arten \\
\hline \multicolumn{5}{|l|}{ Pflanzen } \\
\hline \multirow[t]{2}{*}{ Samenpflanzen } & Etabliert & 432 & 37 & 35 \\
\hline & Unbeständig & $\sim 1.600$ & & 7 \\
\hline \multirow[t]{2}{*}{ Farne } & Etabliert & 1 & 1 & \\
\hline & Unbeständig & 2 & & \\
\hline \multirow[t]{2}{*}{ Moose } & Etabliert & 8 & 1 & \\
\hline & Unbeständig & 3 & & \\
\hline \multicolumn{5}{|l|}{ Wirbeltiere } \\
\hline \multirow[t]{2}{*}{ Fische } & Etabliert & 16 & 4 & 7 \\
\hline & Unbeständig & 21 & 4 & 6 \\
\hline \multirow[t]{2}{*}{ Amphibien } & Etabliert & 2 & 2 & \\
\hline & Unbeständig & 8 & 1 & \\
\hline \multirow[t]{2}{*}{ Reptilien } & Etabliert & 0 & & \\
\hline & Unbeständig & 10 & 1 & 3 \\
\hline \multirow[t]{2}{*}{ Vögel } & Etabliert & 17 & & 8 \\
\hline & Unbeständig & 55 & 3 & 1 \\
\hline \multirow[t]{2}{*}{ Säugetiere } & Etabliert & 11 & 5 & 3 \\
\hline & Unbeständig & 6 & & \\
\hline
\end{tabular}

Das Hauptanliegen des Naturschutzes ist die Bewahrung der Biodiversität. Um dieses Ziel zu erreichen bedarf es klarer Grundlagen und Kriterien, an denen sich mögliche Gefahren durch gebietsfremde Arten erkennen lassen. Diese sollen im Folgenden näher erläutert werden.

\subsubsection{Einführung von Neophyten}

Neophyten wurden und werden auf sehr unterschiedlichen Wegen verbreitet, wobei die wild in Deutschland lebenden invasiven Gefäßpflanzen zu $95 \%$ vom Menschen beabsichtigt eingeführt wurden (Nehring et al., 2013).

Als bedeutendster Einfuhrvektor gilt der Gartenbau und meist treten mehrere Einfuhrvektoren gleichzeitig auf. Somit ist, in Kombination mit der Verwilderung von Zierpflanzen aus Botanischen Gärten, der Gartenbau mitverantwortlich für zwei Drittel der Einbringung von invasiven und potentiell invasiven Gefäßpflanzen in Deutschland. Weitere bedeutende Vektoren sind der Tierhandel (bspw. Aquarien-, Gartenteichpflanzen) und die Forstwirtschaft. (Nehring et al., 2013)

${ }^{13}$ Stand 30.04.2015. 
$\mathrm{Zu}$ ca. $48 \%$ stammen die invasiven und potenziell invasiven Arten aus nordamerikanischen Herkünften. Es wird angenommen, dass das Invasivitätspotential dieser Arten durch die ähnlichen klimatischen Verhältnisse im Vergleich zu ihren ursprünglichen Gebieten bestimmt wird. Vermutlich ebenfalls vom Klima abhängig ist auch die Tatsache, dass es in Deutschland keine invasiven Arten aus dem Tropischen Asien gibt. (Nehring et al., 2013)

\subsubsection{Erstnachweis von Neophyten}

Vom Zeitpunkt der Ersteinbringung eines Neophyten bis zum erstmaligen Nachweis können viele Jahre bis Jahrzehnte vergehen. Natürlich hängt dies direkt mit der Wissensverbreitung über die Artenkenntnisse und den Bemühungen um floristische Kartierungen in den jeweiligen Gebieten zusammen. Im Laufe der Jahrhunderte waren diese Kenntnisse und Bemühungen unterschiedlich ausgeprägt und wurden auch mehrfach durch kriegerische Handlungen bspw. Erster und Zweiter Weltkrieg behindert. Hinzu kommt noch eine meist mehrjährige Verzögerung, bis das Wissen aus Fachzeitschriften öffentlich zugänglich wird und in die Praxis Einzug findet. (Nehring et al., 2013)

In der Invasionsökologie gehen Pyšek \& Jarošík (2005) davon aus, dass die Wahrscheinlichkeit der Ausbreitung einer Art mit der Dauer der Anwesenheit einer Art in dem jeweiligen Gebiet steigt. Diese Ausbreitung kann sich aber zeitlich erheblich verzögern, was laut Nehring et al. (2013) als „time lag“ (engl. für Verzögerungszeit) bezeichnet wird. Im Durchschnitt liegt dieses „time lag“ bei gebietsfremden Gefäßpflanzen in Deutschland bei 129 Jahren. Bei Baumarten verzögert sich der Zeitpunkt von ihrer erstmaligen Einbringung bis zu ihrer natürlichen Verbreitung im Durchschnitt um 170 Jahre (Nehring et al., 2013). Laut Kowarik (1995) ist dies auf die langsameren Generationszyklen von Gehölzen im Vergleich zu bspw. Sträuchern (131 Jahre) zurückzuführen.

Im BfN-Skript 352 (Naturschutzfachliche Invasivitätsbewertungen für in Deutschland wild lebende gebietsfremde Gefäßpflanzen; Nehring et al., 2013) wird beschrieben, dass das „time lag“ für invasive (74 Jahre) und potenziell invasive Neophyten (99 Jahre) geringer ausfällt, als bei allen übrigen Arten (135 Jahre) für die die Daten vorliegen. Begründet wird diese Beobachtung mit der großen Anpassungsfähigkeit von invasiven Neophyten, bspw. ihr erhöhtes Ausbreitungspotenzial durch flugfähige Samen bei einer gleichzeitig hohen Samenproduktion.

\subsubsection{Ausbreitungspotential von Neophyten in Deutschland}

Bei der Hälfte aller invasiven Gefäßpflanzen in Deutschland zeigt sich in den letzten 5 10 Jahren eine starke Ausbreitung, allerdings mangelt es diesbezüglich auch bei vielen invasiven und potenziell invasiven Arten an Informationen. Die Autoren des BfN-Skriptes 352 (Nehring et al., 2013) verweisen daher auf den $\S 40$ Abs. 2 des BNatSchG-es und die Notwendigkeit zu einem Monitoring über die Bestandesentwicklung dieser Arten.

Es wird davon ausgegangen, dass sich ca. zwei Drittel der invasiven Gefäßpflanzen großräumig in Deutschland ausgebreitet haben. Um den negativen Einfluss dieser Arten zu minimieren, erscheint ein lokales Management am sinnvollsten (siehe diesbezüglich auch $\S 40$ Abs. 3 Satz 2 BNatSchG). (Nehring et al., 2013) 
Das eigentliche Ausbreitungspotential lässt sich durch eine Schätzung von Rauer et al. (2000) erahnen, der davon ausgeht, dass ca. 50.000 Pflanzenarten, von denen die Mehrzahl gebietsfremd ist, in Deutschlands Botanischen Gärten kultiviert werden. Nach Kowarik (2010) wachsen allein in den deutschen Parks und Gärten ca. 3.150 verschiedene Gehölzarten. Davon konnten über 2.400 als wild in Deutschland lebende gebietsfremde Arten nachgewiesen werden (BfN, 2012). Als etabliert, gelten 432 gebietsfremde Arten (BfN, 2012), was, bezogen auf die gesamte Flora an terrestrischen und aquatischen Gefäßpflanzen, einem Artenanteil von $11 \%$ entspricht (Nehring et al., 2013).

\subsection{Zur Einführung von gebietsfremden Baumarten}

In Mitteleuropa gab es seit dem 16. Jahrhundert einen wiederkehrenden und örtlich unterschiedlich stark ausgeprägten Mangel an dem elementaren Rohstoff Holz. Ab wann dieser Mangel zum ersten Mal deutlich in Erscheinung trat, wird in der Literatur verschiedenartig diskutiert (siehe hierzu König \& Radkau, 2013). Diese in der Fachliteratur oft als die „Zeit der Holznot“ bezeichnete Phase, erreichte Mitte des 18. Jahrhunderts ihren Höhepunkt und geht einher mit gesellschaftlichen Strömungen wie dem „Naturkult“ der Romantik und der Entstehung der bis heute aktiven Umweltbewegung (Radkau, 2007).

Um diese Not zu lindern und die ausgeplünderten deutschen Wälder wieder zu stabilisieren (Radkau, 2007), begann 1880 der Baumschulbesitzer John Booth mit dem ersten systematischen Anbauversuch von fremdländischen Baumarten (Vor et al., 2015). Es sollte bspw. herausgefunden werden, ob die eingeführten Arten auf vergleichbaren Standorten mehr Masse- bzw. Wertleistung erbringen können und wie hoch ihre Widerstandsfähigkeit gegenüber Witterungsextremen ist. Die deutschen forstlichen Versuchsanstalten etablierten kurze Zeit später ähnliche Versuchsaufbauten, bei denen sie viele Erkenntnisse sammelten, aber eine der wesentlichen Fragen nach der Anbauwürdigkeit der Fremdländer nicht abschließend klären konnten.

Daraufhin wurden neue, größere Versuchsanbauten angelegt und die wissenschaftliche Fragestellung angepasst. Die aus innen abgeleiteten Ergebnisse dienen den heutigen Beurteilungen zur Anbauwürdigkeit einer Vielzahl von fremdländischen Baumarten. (Vor et al., 2015)

Sowohl über die Potentiale als auch die Risiken, die durch die Einbringung von nichtheimischen Baumarten entstehen, wird mindestens seit den ersten Anbauversuchen des ausgehenden 19. Jahrhunderts gestritten. Doch wie die Autoren Vor et al. (2015) feststellen, ging es „beim Anbau eingeführter Baumarten [...] zu keiner Zeit darum, die erdgeschichtlich bedingte Artenarmut in Mitteleuropa wesentlich zu verändern, sondern lediglich um eine Bereicherung der schmalen Baumartenpalette durch einige wenige, anbauwürdige Baumarten unter Beachtung der rechtlichen Rahmenbedingungen." Das sich daraus abzeichnende Spannungsfeld zwischen Naturschutz und Forstwirtschaft verlangt beiderseitige Kompromisse, auf dass der Anbau von nicht invasiven Baumarten akzeptiert wird und dabei naturschutzfachliche Interessen ausreichend Berücksichtigung finden.

Invasive Arten die nach $\S 7$ BNatSchG heimische Arten deren Biotope oder gar Ökosysteme gefährden, werden sowohl vom Naturschutz als auch von der Forstwirtschaft als nicht anbauwürdig eingeschätzt. (Vor et al., 2015) 


\subsubsection{Rechtliche Rahmenbedingungen und Definitionen}

\subsubsection{Internationale Vereinbarungen}

Das Übereinkommen über den internationalen Handel mit gefährdeten Arten freilebender Tiere und Pflanzen, das Washingtoner Artenschutzübereinkommen (CITES = Convention on International Trade in Endangered Species of Wild Fauna and Flora) von 1973, bildet die weltweite vertragliche Grundlage im internationalen Handel mit geschützten Tier- und Pflanzenarten. Das CITES-Abkommen hat aber keine direkte gesetzgebende Autorität und so obliegt es jedem Mitgliedstaat die Inhalte rechtlich umzusetzen. (CITES.org, 2020)

Bei der Konferenz der Vereinten Nationen (UN = United Nations) über Umwelt und Entwicklung 1992 in Rio, wurde das Übereinkommen über die biologische Vielfalt (CBD = Convention on Biological Diversity) unterzeichnet.

Das CBD schreibt erstmalig völkerrechtlich bindend die Ziele und Aufgaben des Naturschutzes fest, zu denen in $\S 8$ auch die Vorsorge, Kontrolle und Bekämpfung invasiver Arten gehört (CBD Secretariat (UN), 2020).

Bei einer Folgekonferenz der UN im Jahre 2000 verpflichteten sich die beteiligten Staaten zur Entwicklung nationaler Strategien auf Grundlage des CBD. Bei der 6. Vertragsstaatenkonferenz von 2002 wurde eine Maßnahmenkatalog verabschiedet, der als Muster für die Umsetzung der nationalen Strategien dienen sollte (siehe hierzu auch „Guiding Principles on Invasive Alien Species“, CBD Secretariat (UN), 2002).

\subsubsection{Europäische Ebene}

Das Washingtoner Artenschutzübereinkommen wurde seitens der Europäischen Union (EU), mittels der Artenschutzverordnung (EG 338 / 97), umgesetzt. Es ermöglicht den europäischen Staaten Einfuhrbeschränkungen für die Arten zu erlassen, die eine ökologische Gefahr für die einheimischen Tier- und Pflanzenarten darstellen (siehe hierzu Art. 3 Abs. 2 d). (BfN, 2020a)

Das erste Übereinkommen über die Erhaltung der europäischen wildlebenden Pflanzen und Tiere und ihrer natürlichen Lebensräume (Berner Konvention $=$ Convention on the Conservation of European Wildlife and Natural Habitats) wurde als völkerrechtlicher Vertrag seitens des Europarates bereits 1979 verabschiedet (BfN, 2020b).

Zur Umsetzung der Konvention wurde die Fauna-Flora-Habitat-Richtlinie (FFHRichtlinie, 1992) verabschiedet. Sie regelt den Aufbau von einem Netz aus Schutzgebieten von besonderer Bedeutung (siehe hierzu auch Natura 2000).

Die Europäische Strategie im Umgang mit invasiven Arten wurde, im Rahmen der Berner Konvention, im Jahr 2003 erarbeitet und im darauffolgenden Jahr vom Europarat veröffentlicht (BfN, 2020b).

Die Europäische Kommission hat 2011 die EU-Biodiversitätsstrategie für 2020 vorgelegt, in welcher sich der Punkt 5, der Bekämpfung invasiver gebietsfremder Arten widmet (EUBiodiversitätsstrategie, 2011; siehe auch Vor et al., 2015).

Darauf aufbauend wurde 2014 die EU-Verordnung Nr. 1143 / 2014 über die Prävention und dem Management zur Einbringung und Ausbreitung invasiver gebietsfremder Arten verabschiedet. Diese Verordnung hat eine Auflistung von Arten mit unionsweiter Bedeutung (Unionsliste) zum Ziel. Auch Maßnahmen wie Prävention, Früherkennung, Reaktion und Kontrolle sind darin festgeschrieben. 


\subsubsection{Nationale Ebene}

Die Umsetzung der vorab genannten europäischen Richtlinien in nationales Recht erfolgt über das Bundesnaturschutzgesetz (BNatSchG, 2009).

Durch das Inkrafttreten des Gesetzes zur Durchführung der Verordnung (EU) Nr. 1143 / 2014, im September 2017, kommt es zu einer Neuregelung über die Thematik der invasiven Arten im BNatSchG (siehe hierzu § 7 und § 40a BNatSchG).

Auf Grundlage des BNatSchG wurde die Bundesartenschutzverordnung (BArtSchV, 2020) zum Schutz wildlebender Tier- und Pflanzenarten erlassen. Sie basiert auf der europäischen Verordnung (EG) Nr. 338 / 97 (Artenschutzverordnung) und enthält in der Anlage 1 eine Liste der geschützten Pflanzen und Tiere, die aber nicht mit der Roten Liste verwechselt werden sollte, die vom Bundesamt für Naturschutz (BfN) herausgegeben wird.

Weitere Gesetze, die sich auf nationaler Ebene mit invasiven bzw. gebietsfremden Arten befassen sind:

- Bundesjagdgesetz (siehe hierzu § 28 Abs. 3 zur Ansiedlung fremder Tiere in der freien Natur)

- Sortenschutzgesetz (Saatgutverordnung)

- Pflanzenschutzgesetz

- Tierseuchengesetz

- Tierschutzgesetz

\subsection{Gesetzliche Definition}

Das BNatSchG (2009) definiert im § 7 Abs. 2 Nr. 9 den Begriff „invasive Art“ und verlangt in Form einer Einzelfallbeurteilung die Prüfung der zwei Tatbestandsmerkmale:

- Ob die Art außerhalb ihres natürlichen Verbreitungsgebietes vorkommt und

- ob von dieser Art ein erhebliches Gefährdungspotential für die heimischen Ökosysteme, Biotope und Arten ausgeht.

Die Autoren Vor et al. (2015) bemerken hierzu, dass die Begriffe „erheblich“ und "Gefährdungspotential“ im Gesetz nicht näher erläutert werden.

Sie gehen weiter davon aus, dass durch den Begriff "Gefährdungspotential“ ein genereller Schadenseintritt nicht erforderlich ist, sondern bereits die Möglichkeit einer Schädigung ausreichend ist. Im weiteren Gesetzestext werden die Rechtsgüter (Ökosysteme, Biotope und Arten) benannt, die bei einer möglichen Schädigung gefährdet wären.

Es kann davon ausgegangen werden, dass der Gesetzgeber mit dem Begriff „erheblich“ bewusst eine zusätzliche Steigerung eingebaut hat und folglich nicht jedes Gefährdungspotential ausreichend ist, um das Tatbestandsmerkmal zu erfüllen.

Da sich keine einheitliche, alle Gesetzte übergreifende Definition von „Erheblichkeit“ finden lässt, raten Vor et al. (2015) dazu „[...] den Begriff [...] durch Betrachtung anderer Stellen im BNatSchG unter Heranziehung der diesbezüglichen Kommentarliteratur auszufüllen." Die Autoren kommen zu dem Schluss, dass ein erhebliches Gefährdungspotential "[...] mehr als die bloße Gefährdung der genannten [Rechts-]Güter" erfordert und dass bei dem Begriff der Erheblichkeit Interpretationsbedarf besteht." Sie stellen abschließend folgende Definition auf: 
„Invasiv ist eine Art dann, wenn sie durch das Auftreten außerhalb ihres ursprünglichen Verbreitungsgebiets die Schutzgüter Ökosysteme, Biotope oder Arten zwar nur möglicherweise, im Falle des Eintretens aber merklich und zumindest von gewisser Dauer gefährden kann. Das ist dann der Fall, wenn heimische Arten sich auf Dauer nicht mehr durchzusetzen vermögen bzw. Ökosysteme oder Biotope sich auf Dauer nachteilig verändern oder gar verschwinden." (Vor et al., 2015)

Wenn eine Tier- oder Pflanzenart die vorab genannten zwei Tatbestandsmerkmale erfüllt so sieht das BNatSchG die im Folgenden aufgezählten Rechtsfolgen vor (siehe hierzu § 40a BNatSchG):

- Im Falle von Gefährdungen, die Einleitung von geeigneten Maßnahmen ( 40a BNatSchG und $\S 48 a$ BNatSchG).

- Eine Beobachtungspflicht bei Arten die Anzeichen von Invasivität zeigen ( 40b BNatSchG).

- Beseitigungsmaßnahmen (§ 40a Abs. 3 S. 1 BNatSchG).

- Maßnahmen zur Verminderung der Auswirkungen durch invasive Arten (§ 40d BNatSchG).

Wenn es sich jedoch um den Anbau von Pflanzen in der Land- und Forstwirtschaft handelt, so gelten die vorab benannten Beseitigungs- und Verminderungsmaßnahmen nicht (§ 40 Abs. 1 S. 1 BNatSchG). Denn eine Beseitigungsanordnung kann gemäß $\S$ 40a Abs. 3 BNatSchG nur dann ausgesprochen werden, insofern sich die invasive Art unbeabsichtigt in der freien Natur ausbreitet und eine Gefährdung vorliegt (siehe hierzu auch Vor et al., 2015).

\subsubsection{2 Ökologische Definition}

Definitionen in den Naturwissenschaften unterscheiden sich insofern von Gesetzestexten und naturschutzfachlicher Literatur, dass sie zumeist frei von Wertungen sind. So verwundert es nicht, dass in der Ökologie die Begrifflichkeiten „Invasion“ und „invasive Art" sehr unterschiedlich verwendet werden.

Eine eher negative Notation des Begriffes der Invasion findet sich schon im Jahr 1958 in dem Aufsatz: „The ecology of invasions by animals and plants“ von Elton et al. (1958). Spätestens aber seit der Umweltkonferenz von Rio 1992 wird eine Bekämpfung von invasiven Arten gefordert, da von ihnen eine Gefährdung der biologischen Vielfalt ausgehen kann. In neuerer Zeit sind noch Betrachtungen der gesundheitlichen und ökonomischen Folgen von Invasivität hinzugekommen und so definieren bspw. Weber \& Broennimann (2013) invasive Arten in Form von sich rasch ausbreitenden und zu massenhaftem Vorkommen neigende verwilderte Arten, die wiederum negative Auswirkungen auf Mensch und Umwelt zur Folge haben. In dem BfN-Skript 213 (Hubo et al., 2007) werden die Begrifflichkeiten „Invasion“ und „invasive Art“ in aller Ausführlichkeit besprochen.

Eine der am häufigsten in der Literatur verwendeten Definitionen ist die von Kowarik (2010) nach der unter einer Invasion das Folgende verstanden wird:

„Invasion: [ist ein] Durch Menschen ermöglichter Prozess der Vermehrung und Ausbreitung von Organismen in Gebieten, die sie auf natürliche Weise nicht erreicht haben." Vor et al. (2015) merken an, dass nach dieser Definition auch nahezu alle einheimischen Baumarten als invasiv gelten müssten. 


\subsubsection{Bewertungskriterien zur Invasivität}

\subsubsection{Naturschutzfachliche Bewertungskriterien}

In der von Nehring et al. (2015) erstellten „Methodik der naturschutzfachlichen Invasivitätsbewertung für gebietsfremde Arten" werden die folgenden fünf Hauptkriterien nach denen sich eine mögliche Gefährdung der heimischen Biodiversität unterscheiden lassen, benannt:

- Interspezifische Konkurrenz

- Negative ökosystemare Auswirkungen

- Hybridisierung

- Krankheits- und Organismenübertragung

- Prädation und Herbivorie

Die Gefährdung heimischer Arten durch invasive Gefäßpflanzen wird hauptsächlich durch die beiden erstgenannten Kriterien, der interspezifischen Konkurrenz und der negativen ökosystemaren Auswirkungen verursacht. Diese meist zusammen auftretenden Hauptwirkfaktoren führen auch zur Ausbildung von Dominanzbeständen seitens der invasiven Arten. (Nehring et al., 2013)

Zwischen heimischen und gebietsfremden Arten kann es auch zu Hybridisierungen kommen, aus denen fertile Hybriden hervorgehen. Die Autoren Nehring et al. (2013) vermuten, dass dieses Kriterium ein bei weitem größeres Gefahrenpotenzial besitzt als es bisher bekannt war, da nach Schmitz et al. (2008) die Auswirkungen solcher Hybridisierungen auf die heimische Flora bisher nur unzureichend betrachtet wurden.

Durch die letzten beiden Kriterien der Krankheits- und Organismenübertragung und der Prädation und Herbivorie konnten bisher keine erheblichen Gefährdungen der heimischen Biodiversität durch gebietsfremde Arten festgestellt werden (Nehring et al., 2013).

Durch den fortschreitenden Klimawandel wird die Mehrheit der invasiven und potenziell invasiven Gefäßpflanzen gefördert. Es ist nach Ansicht der Autoren Nehring et al. (2013) davon auszugehen, dass sich Neophyten von den Ökosystemen menschlicher Ballungsräume stärker in ländliche Regionen ausbreiten werden. Allerdings ist für ca. ein Drittel der invasiven Arten keine Einschätzung zur Förderung durch den Klimawandel möglich, da es an Daten mangelt.

\subsection{Kritik seitens der Forstwissenschaften}

Das Bundesnaturschutzgesetz bildet in der Bundesrepublik Deutschland den rechtlichen Rahmen zum Naturschutz und der Landschaftspflege. Im § 40 wird die Ausbringung von Pflanzen und Tieren geregelt, „[...] deren Art in dem betreffenden Gebiet in freier Natur nicht oder seit mehr als 100 Jahren nicht mehr vorkommt [...]“, und daher von der zuständigen Behörde genehmigungspflichtig ist.

Die Land- und Forstwirtschaft sind beim Anbau von Pflanzen von den „[...] Erfordernissen einer Genehmigung [...] ausgenommen“. 
Das BNatSchG bewegt sich dabei in dem größeren gesetzlichen Rahmen, welcher von der EU vorgegeben wird und somit sind seitens der EU die Verordnung (EU) Nr. 1143 / 2014 des Europäischen Parlaments und des Rates zur Prävention und Management bei der Einbringung und Ausbreitung invasiver gebietsfremder Arten, sowie Artikel 22 der Richtlinie 92 / 43 / EWG zu beachten. (BNatSchG, 2009)

Das Bundesamt für Naturschutz $(\mathrm{BfN})$ ist dem Bundesministerium für Umwelt, Naturschutz und nukleare Sicherheit (BMU) nachgeordnet und unterstützt und berät dieses in Fragen der Landschaftspflege, des Naturschutzes und bei der internationalen Zusammenarbeit (BfN, 2020a).

Im November 2013 wurde seitens des BfN das Skript 352 „Naturschutzfachliche Invasivitätsbewertungen für in Deutschland wild lebende gebietsfremde Gefäßpflanzen“ (Nehring et al., 2013) veröffentlicht. Es wird darin das Ziel verfolgt, gebietsfremde Arten bezüglich ihrer Verbreitung, weiteren Bestandesentwicklung und der daraus resultierenden möglichen Gefährdung der biologischen Vielfalt zu überwachen. Jegliche Maßnahmen gegen diese gebietsfremden Arten sollen zur Minimierung ihres negativen Einflusses auf das heimische Ökosystem beitragen.

Mittels des Deutschen Verbandes Forstlicher Forschungsanstalten (DVFFA, 2020) äußerten 21 Forstwissenschaftler, in einem offenen Brief (Ammer et al., 2014), Zweifel an der Invasivitätsbewertung im BfN-Skript 352 (Vor et al., 2015).

Sie kritisieren die:

- Auswahl von Schadensindikatoren,

- Unzureichende Berücksichtigung ausbreitungsbiologischer Eigenschaften,

- Invasivitätseinstufung bei den Baumarten,

- Unvollständige Berücksichtigung der Fachliteratur,

- Verallgemeinerung von lokalen Gefährdungen der Biodiversität

und die „[...] einseitige Betrachtung von Ursache / Wirkungs-Beziehungen bei der Gefährdung der Arten." Von den Unterzeichnern des Briefes wurde daraufhin eine eigene Bewertung angekündigt, die sich auf Maßnahmen zur Kontrolle und auf die Begrenzung zur Ausbreitung von gebietsfremden Arten konzentriert.

\subsubsection{Forstliche Bewertungskriterien}

Nach Ammer et al. (2014) ist eine Art als invasiv zu beurteilen, wenn die von ihr ausgehende Gefährdung real und nachweisbar ist und diese sich nicht durch waldbauliche oder andere Möglichkeiten steuern lässt, bzw. diese Möglichkeiten gänzlich fehlen oder zu aufwendig wären.

Die Einschätzung ob eine Gefährdung vorliegt, kann nach Vor et al. (2015) nur erfolgen, wenn die von der Art ausgehenden populationsbiologischen Eigenschaften, wie Ausbreitungs-, Konkurrenz- und Reproduktionsverhalten und deren Wirkung auf den Standort, berücksichtigt werden. Eines der wichtigsten Kriterien ist hierbei das Management, womit die gezielte Steuerung der Ausbreitung der Art gemeint ist.

Die Autoren Vor et al. (2015) beschreiben fünf Kriterien zur Bewertung der Invasivität, wobei sie sowohl verjüngungsökologische als auch ökosystemare Aspekte berücksichtigen: 
- Negative Standortbeeinflussung $=$ negative Beeinträchtigung der Besiedelungs- und Lebensbedingungen für heimische Arten.

- Hohes Reproduktionspotenzial = frühe und starke generative bzw. vegetative Vermehrung und Etablierung überlebensfähiger Jungbestände.

- Hohes Ausbreitungspotential = Fähigkeit zur schnellen und großflächigen Besiedlung von Lebensräumen, in starker Abhängigkeit vom Reproduktionspotenzial.

- Fähigkeit zur Artenverdrängung = Bildung von Reinbeständen durch hohe Konkurrenzkraft und schlechte Mischungsmöglichkeiten mit heimischen Baumarten.

- Begrenzte Steuerungsmöglichkeiten = wirksame, ökologisch vertretbare und finanzierbare Regulierung auf biologischer, mechanischer oder chemischer Ebene.

Erst die Betrachtung aller Kriterien kann Aufschluss über die Invasivität einer Art geben, wobei das Ausbreitungspotenzial in Kombination mit der Fähigkeit zur Artenverdrängung besonders gewichtig ist. Speziell aber die Möglichkeit zur Steuerung ist maßgebend für die Anbauwürdigkeit einer nichtheimischen Art in deutschen Wäldern. (Vor et al., 2015)

\subsubsection{Bewertung der Invasivität von P.tomentosa}

Eine einheitliche Invasivitätsbewertung der Gattung Paulownia ist nicht möglich, da die einzelnen Arten bspw. bezüglich dem Ausbreitungspotential und dem Einsetzen der Verjüngung sehr unterschiedlich sind.

Da die Art P.tomentosa weltweit am stärksten verbreitet ist und auch am häufigsten angebaut wird, erscheint eine Bewertung dieser Art sowohl aus naturschutzfachlicher als auch forstlicher Sicht am sinnvollsten (1.1.4 Die „Hauptbaumart“ P.tomentosa).

\subsubsection{Naturschutzfachliche Invasivitätsbewertung}

Das BfN-Skript 352 (Nehring et al., 2013) widmet sich auf anderthalb Seiten der naturschutzfachlichen Invasivitätsbewertung der P.tomentosa, welches im Anhang zu Kapitel II (Abb. 48: Naturschutzfachliche Invasivitätsbewertung von P.tomentosa, entnommen und verändert nach Nehring et al. (2013) zu finden ist und im Folgenden auszugsweise näher besprochen wird.

\subsection{Hauptkriterien zur Invasivitätsbewertung}

Eine Gefährdung der Biodiversität im Bereich der Interspezifischen Konkurrenz wird als unbekannt eingestuft, da sich Erfahrungen aus den USA nicht einfach auf Deutschland übertragen lassen. Nach Remaley (2005) kommt es nämlich in amerikanischen Wäldern nach Störungen wie bspw. großflächigen Bränden zwischen Paulownia und den heimischen Arten zur Konkurrenz bei der Neubesiedlung des Biotops.

Sowohl bei der Hybridisierung als auch bei der Krankheits- und Organismenübertragung wird eine Gefährdung von heimischen Arten verneint und eine Gefahr durch Prädation und Herbivorie kann nicht beurteilt werden. Auch mögliche negative ökosystemare Auswirkungen werden als unbekannt gewertet. (Nehring et al., 2013) 


\subsection{Zusatzkriterien zur Invasivitätsbewertung}

Die aktuelle Verbreitung in Deutschland wird als kleinräumig beschrieben (Essel, 2007; Keil \& Loos, 2004; Richter \& Böcker, 2001) und sowohl eine mechanische als auch chemische Bekämpfung erscheinen möglich (Remaley, 2005).

Das Vorkommen von Paulownia „[...] in natürlichen, naturnahen und sonstigen naturschutzfachlich wertvollen Lebensräumen“ wird bestätigt, wobei laut Essel (2007) diese nur sehr selten bspw. an Flussufern und auf Waldlichtungen zu beobachten sind. Das Reproduktionspotential (Remaley, 2005) und das damit einhergehende Ausbreitungspotential wird als hoch und der aktuelle Ausbreitungsverlauf als expansiv eingestuft (Essel, 2007; Keil \& Loos, 2004). Es wird angenommen dass der voranschreitende Klimawandel das Invasionsrisiko durch die Baumart fördert (Essel, 2007). Es besteht weiterer Forschungsbedarf um die Wissenslücken hinsichtlich „langfristige( $r$ ) Invasivitätsrisiken in naturnahen Lebensräumen“ zu schließen. (Nehring et al., 2013; siehe hierzu auch Hubo et al., 2007)

\subsubsection{Versuch einer forstlichen Invasivitätsbewertung}

\subsubsection{1 Ökologische Integration der Baumart}

Aufgrund der Größe, Schönheit und Vielzahl der Blüten ist die Baumart schon seit Jahrzehnten in vielen Gärten zu finden. So verwundert es nicht, dass sich viele verschiedene pollen- und nektarfressende Insektenarten der Blüten zur Nahrungsgewinnung bedienen. (Innes, 2009)

Die Blüten und Blätter werden aufgrund ihres hohen Zuckergehaltes in China gerne als Viehfutter verwendet (Zhu et al., 1986). Allerdings führt dies auch zu Verbissschäden durch verschiedene Säugetierarten, wie Untersuchungen aus Nordamerika gezeigt haben (Innes, 2009).

Kobayashi et al. (2008) konnten nachweisen dass die Art P.tomentosa eine ganze Reihe von Mechanismen zu Abwehr von Herbivoren besitzt. Beispielsweise finden sich an ihren Blättern, Stielen und Blüten feine Härchen die Glyceride enthalten und an denen Insekten festkleben. Extraflorale Nektarien an den Blütenknospen bilden ein zuckerhaltiges Sekret aus, welches von Ameisen genutzt wird, die wiederum Schadinsekten abwehren.

\subsection{Krankheiten und Prädatoren}

Im natürlichen Verbreitungsgebiet der Baumart gibt es eine ganze Reihe von Krankheiten und Schädlinge (Tab. 10). Diese treten besonders bei großflächigen Anpflanzungen wie bspw. bei Baumschulen und Plantagen in Erscheinung und deren Ausbreitung bzw. Vermehrung lässt sich durch ein verbessertes Management beim Anbau der Arten verringern. (Zhu et al., 1986)

Auch invasive Arten wie bspw. die Wanzenart Halyomorpha halys, die ursprünglich aus Ostasien stammt, nutzt in Europa angepflanzte Paulownia-Pflanzen als Nahrungsgrundlage (Wermelinger et al., 2008). 


\subsection{Ausbreitungspotential}

In Nordamerika, wo Paulownia nach großflächigen Störungen wie bspw. Feuer als stark invasiv gilt, kommt es bereits auf nährstoffarmen und trockenen Standorten zur Verdrängung von geschützten einheimischen Pflanzenarten (Innes, 2009). Dieser erhöhte Invasivitätsdruck ist vermutlich auf die großflächigen Paulownia-Anbauten der 1970er und 80er Jahre zur Wertholzgewinnung zurückzuführen (Innes, 2009; Zhu et al., 1986).

In Mitteleuropa verbreitet sich die Baumart, unter natürlichen Bedingungen, bevorzugt auf Extremstandorten, die nur von wenigen anderen einheimischen Arten besiedelt werden können. Daher sind laut Essel (2007) naturnahe Ökosysteme von einer Ausbreitung bisher nicht betroffen.

Richter \& Böcker (2001) hingegen berichten von Beobachtungen aus der Schweiz, wonach sich die Art in Niederwäldern und auf Waldlichtungen ausbreitet.

Es ist vorstellbar dass zukünftig auch naturnahe Ökosysteme in Mitteleuropa stärker unter Druck geraten, da sich die Anbaufläche von ursprünglich nur in Gärten und Parks vorhandenen Einzelbäumen auf großflächige Plantagenwirtschaft in Form von KUPs und Agroforst deutlich erhöht (Cathaia, 2020; Essel, 2007; Thielen, 2019).

Die durch den Klimawandel verstärkt vorkommende Trockenheit und die höheren Sommertemperaturen werden von der Paulownia toleriert, bzw. letztere ist gar förderlich für das Wachstum. Daraus kann sich ein erhöhtes Ausbreitungspotential und somit eine Gefährdung bzw. Verdrängung heimischer Arten ergeben. (Vor et al., 2015)

\subsection{Steuerungsmöglichkeit}

P.tomentosa ist eine früh-sukzessionale Art die schnell von später auftretenden, deutlich schattentoleranteren Arten verdrängt wird (Vor et al., 2015).

Durch ihren Pioniercharakter und hohe Wachstumsrate eignet sich die Art zur Rekultivierung von ehemaligen Tagebauflächen, wo sie dem Erosionsschutz dient und durch den hohen Stickstoffgehalt der Laubstreu zur Bodenverbesserung beitragen kann. Eine ganze Anzahl von Hybridisierungen ist, wie in Kapitel I (1.1.1.2 Natürliche Verbreitung) bereits erläutert, aus dem natürlichen Verbreitungsgebiet bekannt. Darüber hinaus gibt es weitere Züchtungen zur Anpassung an die mitteleuropäischen Wachstumsbedingungen. (Cabi, 2019; Stimm et al., 2013)

P.tomentosa ist auch als Jungpflanze vergleichsweise konkurrenzschwach gegenüber anderen Arten, wenn sie es nicht schafft die umgebende Bodenvegetation mittels ihres Blätterdaches frühzeitig auszudunkeln. Ihre Blattstreu verringert dabei sogar die Keimungsrate anderer Arten. In geschlossenen Beständen kann sie sich nicht mehr regenerativ verjüngen, da sie zur Keimung auf offenes Bodensubstrat ohne Streu angewiesen ist. (Innes, 2009)

\subsection{Fazit zur forstlichen Invasivitätsbewertung}

Bei P.tomentosa ist eine eindeutige Aussage zur Invasivität nicht möglich. Es gibt Anzeichen zu invasivem Verhalten wie bspw. in den USA (Innes, 2009; Remaley, 2005), die sich aber nicht ohne Weiteres auf mitteleuropäische Verhältnisse übertragen lassen (Nehring et al., 2013). 
Die Autoren Vor et al. (2015) betrachten die Baumart abschließend als nicht anbauwürdig in deutschen Wäldern und ermahnen, dass bei einem möglichen Anbau auf landwirtschaftlichen Kurzumtriebsplantagen „[...] eine unerwünschte Ausbreitung in angrenzende Gebiete unterbleibt."

Bei einem Vergleich von P.tomentosa mit Gleditschie (Gleditsia triacanthos), Essigbaum (Rhus typhina) und Robinie (Robinia pseudoacacia) zeigt sich eine ähnliche bzw. gleiche Einteilung anhand der Kriterien nach Vor et al. (2015) (Tab. 11). Allerdings wird diesen drei Arten dann doch eine andere bzw. differenziertere Invasivitäts-Bewertung zuteil als es für P.tomentosa der Fall ist.

Beispielsweise zeigen Gleditschie und Essigbaum nur auf „[...] Offenlandstandorten invasive Tendenzen [...]" und können sich in geschlossenen Wäldern aufgrund ihrer Lichtbedürftigkeit nicht durchsetzen und gelten daher als nicht anbauwürdig in deutschen Wäldern. Die Robinie hingegen wird als anbauwürdig „,...] auf armen, trockenen Waldstandorten und in Kurzumtriebsplantagen im subkontinentalen Bereich [...]" bezeichnet und das, obwohl sie der P.tomentosa bezüglich der Invasivitätskriterien (genau wie der Gleditschie) gleicht. Jedoch muss auch bei der Robinie eine Kontrolle über eine mögliche ungewollte Ausbreitung gewährleistet werden. (Vor et al., 2015)

\subsubsection{Maßnahmen im Umgang mit gebietsfremden Arten}

\subsubsection{Allgemeine Maßnahmen}

Das Bundesamt für Naturschutz unterteilt seine Empfehlungen und Maßnahmen im Umgang mit gebietsfremden Arten in die drei Ebenen: Vorsorge, Monitoring (Früherkennung und Sofortmaßnahmen) und Akzeptanz (Kontrolle und Beseitigung) (Neobiota, 2020).

\subsection{Vorsorge}

Bei der Vorsorge kommt es vor allem auf die Aufklärung und der damit einhergehenden Bewusstseinsbildung an, da Privatpersonen meist einen zu unbewussten Umgang mit gebietsfremden Arten zeigen.

Auch sollte bspw. in der Land- und Forstwirtschaft die Nutzung von einheimischen Arten gefördert werden und die unbeabsichtigte Ausbreitung weitestgehend verhindert werden. Hilfreich hierfür sind die vom Bundesamt für Naturschutz herausgegebenen Skripten mit den rechtlich nicht bindenden "grauen“ und „schwarzen“ Listen und naturschutzfachlichen Invasivitätsbewertungen gebietsfremder Arten (siehe hierfür (Nehring et al., 2013; Neobiota, 2020).

\subsection{Monitoring}

Um rechtzeitige Kontroll- oder Bekämpfungsmaßnahmen durchführen zu können, wie sie im BNatSchG $\S 40 a$ geregelt sind, bedarf es der stetigen Beobachtung zur Bestandesentwicklung gebietsfremder Arten und der Früherkennung im Falle einer ungewollten Ausbreitung. 
Das BfN empfiehlt daher die Etablierung eines Frühwarnsystems unter Mithilfe von Fachexperten, Wissenschaftlern und auch Laien. Ein frühzeitiges Ergreifen von Maßnahmen (§ 40e BNatSchG) wird dadurch möglich und Schäden an der heimischen Flora und Fauna können verhindert werden. Generell „[...] ist die Eindämmung der von invasiven Arten ausgehenden Gefahr umso schwieriger und teurer, je länger man wartet bzw. je weiter sie verbreitet sind." (Neobiota, 2020)

\subsection{Akzeptanz}

Das BfN spricht sich dafür aus, den Großteil der gebietsfremden Arten in Deutschland zu akzeptieren, da sie sich längst als Bestandteile der hiesigen Flora- und Fauna-Welt etabliert haben und nicht mehr ausrottbar sind. Daher soll „nur in begründeten Einzelfällen“ eine Bekämpfung erfolgen bei der bspw. eine „Bedrohung seltener oder gefährdeter Arten oder Lebensräume oder besonders negative Auswirkungen auf den Naturhaushalt, die menschliche Gesundheit oder wirtschaftliche Aktivitäten" zu erwarten sind. Um den damit verbundenen finanziellen Aufwand gering zu halten und die Schäden an anderen Arten zu begrenzen, sollte:

- langfristig die Erhaltung des zu schützenden Lebensraumes gesichert sein,

- alle Maßnahmen mit den Schutzzielen und Bedingungen vor Ort übereinstimmen,

- sichergestellt sein, dass in der Öffentlichkeit durch die Maßnahmen kein „widersprüchliches Bild des Naturschutzes“ entsteht,

- die nötigen Mittel zur Durchführung der Maßnahmen bereitgestellt werden, und eine sich anschließende Beobachtung über die Resultate der Maßnahmen erfolgt. (Neobiota, 2020)

\subsubsection{Maßnahmen im Umgang mit P.tomentosa}

\subsection{Natürliche Bekämpfung}

P.tomentosa ist eine Lichtbaumart und besitzt nur wenig Konkurrenzkraft gegenüber schattentoleranten Arten. Daher wird sie in Folge der natürlichen Sukzession auf den meisten Standorten zurückgedrängt. (Vor et al., 2015)

Laut Moore \& Lacey (2009) kann die zusätzliche Einbringung von Samen einheimischer Arten, die eine höhere Keimungsrate aufweisen, diesen Sukzessionsprozess unterstützen, insofern den einheimischen Arten ein Vorsprung im Wachstum ermöglicht wird und sie somit die Paulownia ausdunkeln.

Nach Remaley (2005) ist die effektivste natürliche Bekämpfungsmaßnahme die Öffentlichkeitsarbeit, auf dass eine absichtliche und unabsichtliche Ausbringung verhindert wird.

\subsection{Mechanische Bekämpfung}

In Abhängigkeit von der Größe der Pflanze bzw. des Baumes ist eine Vielzahl mechanischer Maßnahmen denkbar und frei miteinander kombinierbar.

Wenn es sich um Sämlinge bzw. einjährige Pflanzen handelt, so ist das händische aus dem Boden ziehen die einfachste Methode. Es ist vorteilhaft dies nach einem Regenguss durchzuführen, weil dann der Boden lockerer ist und die Wurzeln sich leichter lösen. Auch muss darauf geachtet werden, dass nach Möglichkeit alle Wurzelteile entfernt werden, weil sonst mit einem erneuten Austrieb zu rechnen ist. (Johnson, 2005) 
Größere Pflanzen, ab dem zweiten Jahr, können am Stammfuß geringelt bzw. abgesägt werden. Die Pflanze wird sehr wahrscheinlich noch in der gleichen oder in der nächsten Vegetationsperiode mittels zahlreicher Schösslinge erneut austreiben. Das erstmalige Ringeln bzw. Absägen ist also nur der Beginn einer ganzen Reihe von Maßnahmen und muss vermutlich mehrfach wiederholt oder mittels chemischer Bekämpfung ergänzt werden. Johnson (2005) empfiehlt das Absägen der Pflanze bzw. des Baumes nachdem die Blüte begonnen hat, weil viel Energie für die Blüte aufgewendet wurde und somit der Neuaustrieb geschwächt wird und um generell die Samenproduktion zu verhindern.

Aufgrund des hohen Stockausschlagvermögens der Paulownia rät Remaley (2005) von Maßnahmen wie Zurückschneiden und Ringeln ab und beschreibt nur das Roden mit gleichzeitigem Entfernen der Wurzeln als effektivste Bekämpfungsmaßnahme.

Im Gegensatz dazu empfiehlt Innes (2009), auf Standorten auf denen sich Paulownia durch Verdrängung der einheimischen Arten dauerhaft etabliert hat, ein kombiniertes Management aus regelmäßigem Zurückschneiden, Ringeln und eine sich anschließende Herbizid-Behandlung der Stöcke, um sowohl die Samenproduktion als auch den Stockausschlag zu minimieren.

\subsection{Chemische Bekämpfung}

Der Einsatz von Herbiziden geht in Deutschland mit einer ganzen Reihe von Vorschriften einher, um den unsachgemäßen Gebrauch zu verhindern und generell den Einsatz von Giften in der Umwelt weitestgehend zu minimieren. Es existieren eine Vielzahl von Produkten auf dem Weltmarkt und ihre Verfügbarkeit und Anwendungsmöglichkeiten unterscheiden sich sowohl zwischen land- wie forstwirtschaftlichen Standorten als auch von Land zu Land.

Eine chemische Bekämpfung der Paulownia mit Herbiziden ist möglich, sollte aber aufgrund der Gefährdung der heimischen Flora und Fauna nur in Einzelfällen oder zur Beseitigung bei großräumigen Störungen erfolgen (Remaley, 2005).

Laut Johnson (2005) erscheint es ratsam nur solche Mittel zu verwenden, bei dem sich sowohl Sämlinge als auch Jungbäume mittels einer Brühe aus Wasser, 0,5\% nichtionischem Tensid und zweiprozentigem Glyphosat (bspw. Roundup) oder Triclopyr (eine Pyridincarbonsäure bspw. Garlon) abtöten lassen, indem alle Blätter besprüht werden.

Bei älteren Bäumen, die zuvor abgesägt wurden und bei denen ein Neuaustreib verhindert werden soll, kann eine anschließende Behandlung des Stubbens mit einer Brühe aus je $50 \%$ Glyphosat (oder Triclopyr) und Wasser erfolgen. Wichtig dabei ist, dass der gesamte Stubben und vor allem sein äußerer Ring benetzt werden und die Anwendung nicht bei Frost geschieht. (Johnson, 2005) 


\subsection{Kurzumtriebsplantagen}

Die Baumarten der Gattung Paulownia werden schon seit geraumer Zeit in verschiedenen Landnutzungssystemen weltweit angebaut. In Deutschland bietet sich für einen kommerziellen P.-Anbau das System der Kurzumtriebsplantage (KUP) an, mit dem die traditionelle Trennung von Land- und Forstwirtschaft überwunden werden kann. Im Grunde geht es hierbei um die Produktion von Holz auf landwirtschaftlicher Fläche, wobei sich im Laufe der Zeit eine große Palette an Produktionssystemen herausgebildet hat, die sich stark in Bezug auf die Anlage, Bewirtschaftung und Ernte von Produkten unterscheiden und die mannigfache Auswirkungen auf die Natur und die Landschaft entfalten (Reeg et al., 2009a). Im Folgenden soll darüber ein Überblick gegeben werden

\subsubsection{Definitionen und Rechtlicher Rahmen}

\subsubsection{Entwicklung und Definition}

Mit dem erstmaligen Anbau von Hybriden aus europäischen (Populus nigra L.) und kanadischen (Populus deltoides L.) Schwarzpappeln, vor über 100 Jahren, wurde die heutige, moderne Form von KUPs in Mitteleuropa etabliert (Dickmann, 2006).

Seitdem werden Pappel-Hybridisierungen mit dem Ziel einer hohe Biomasseleistung bei gleichzeitiger Resistenz gegenüber Schadfaktoren gezüchtet und abhängig vom Design der KUP sowohl zur Energieholz- als auch zur Industrieholzproduktion verwendet. Die zweithäufigste Baumart für mitteleuropäische KUPs ist die Weide und hierbei speziell die Hybriden der Korbweide (Salix viminalis L.), die bei eher kurzer Umtriebszeit bevorzugt zur Gewinnung von Holzhackschnitzeln angebaut werden. (Möndel et al., 2009)

Häufig werden KUPs mit den historisch entstandenen Niederwaldsystemen verglichen (Dickmann, 2006), da es Ähnlichkeiten bei den verwendeten Baumarten, bezüglich des Stockauschlagvermögens und dem Produktionsziel, der Maximierung des Holzertrags, gibt. Größere Unterschiede finden sich jedoch bei der Bewirtschaftungsintensität, was bspw. durch die Definition von Kurzumtriebskulturen nach Drew et al. (1987) verdeutlicht wird, die da lautet: „Ein waldbauliches System [..., welches] auf kurzen Kahlschalgszyklen von meist einem bis 15 Jahren, unter Verwendung intensiver Kulturtechniken wie etwa Düngung, Bewässerung und Unkrautbekämpfung sowie genetisch überlegenen Pflanzenmaterials [basiert]." (übernommen und verändert nach Drew et al., 1987; siehe Knust, 2009)

Historische Niederwälder hingegen bestanden aus züchterisch unveränderten einheimischen Baumarten wie bspw. Hainbuche, Hasel, usw. und wurden meist in zeitlichen Abständen von 15 - 30 Jahren beerntet (Hofmann, 2007). Hinzu kommt noch die ökonomisch durchaus relevante Waldweide als eine Form der Nebennutzung (Möndel et al., 2009).

Dagegen sind KUPs ein stärker von der Land- als von der Forstwirtschaft geprägtes System, da sie eine vergleichsweise hohe Bewirtschaftungsintentsität aufweisen, die meist aus nur einem Klon bestehenden Pflanzverbände geometrisch angelegt werden und es auch zum Einsatz von Dünger und Pflanzenschutzmitteln kommen kann (Möndel et al., 2009). 
Eine in der älteren Literatur häufig verwendete Definition, die sich im Grunde aber auf den Anbau von Fichtenholz bezieht, ist die nach Thomasius (1991): „Baumplantagen, sind der Produktion spezieller Forsterzeugnisse dienende, nach geometrischen Prinzipien geordnete Anpflanzungen besonders dafür geeignete Baumarten, Rassen oder Sorten auf von Natur aus oder durch künstliche Zubereitung sehr produktiven Standorten, die bei hinreichendem Schutz und entsprechender Pflege in kurzen Produktionszeiträumen nach Quantität und / oder Qualität über dem natürlichen Niveau liegende Erträge liefern.“

In jüngerer Zeit hat sich die Definition nach Knust (2009) durchgesetzt, nach derer sind KUPs: „[...] intensive Produktionssysteme zur Holzerzeugung in kurzen Zeiträumen [..., mit] zu diesem Zweck gezüchteten sehr produktiven Baumarten, einer hohen Pflanzdichte und vollmechanisierter Ernte in Abständen von wenigen Jahren“.

\subsection{Kurzumtriebsplantagen in Deutschland}

Die Einführung einer neuen, viele land- und forstwirtschaftliche Aspekte ineinander vereinenden Landnutzungsform, stellt schon aufgrund der traditionellen Trennung der Berufsbilder in der Forst- und Landwirtschaft, der Gesetzgebung und der Verwaltungsstrukturen in Deutschland eine Herausforderung dar.

Im Zusammenhang mit der Debatte um die Versorgungssicherheit mit heimischen Rohstoffen, ausgelöst durch die Ölkrisen von 1973 und 1979 / 80, bekam der Anbau von schnellwachsenden Baumarten, mit dem Ziel der Biomasseproduktion, erstmalig eine größere Bedeutung. Anfang der 1990 er Jahre wurde darüber debattiert die nicht für die Nahrungsmittelproduktion benötigten landwirtschaftlichen Flächen einer sinnvollen Nutzung zuzuführen. (Möndel et al., 2009)

Auf Grund der in den 2000 er Jahren wieder vermehrt diskutierten Frage des Klimaschutzes, erhöhte sich der Druck zur Verwendung erneuerbarer Energiequellen und somit auch zur Kohlendioxid $\left(\mathrm{CO}^{2}\right)$ neutralen Energiegewinnung bspw. aus Biomasse (SRU, 2007). In jüngerer Zeit haben sich die Rahmenbedingungen zur Etablierung von KUPs wieder verschoben.

Die sprunghaften Preissteigerungen von Agrarprodukten, ausgelöst durch die Weltwirtschaftskrise von 2008 / 09, konkurriert bspw. mit den Anreizprogrammen der Bundesregierung zum Aufbau von Biogasanlagen und Biomasseheizkraftanlagen. Somit ergibt sich ein Spannungsfeld zwischen der Akzeptanz und der Bereitschaft zur Etablierung von KUPs in Deutschland.

\subsection{Klimabedingte Potentiale}

Die voranschreitende weltweite Erwärmung, die zurzeit bei über $1^{\circ} \mathrm{C}$ liegt (Lenssen et al., 2019), fällt lokal betrachtet sehr unterschiedlich aus. Für das Pflanzenwachstum sehr wichtige Klimafaktoren sind der Niederschlag, die Temperatur und die Konzentration von $\mathrm{CO}^{2}$ und Ozon $\left(\mathrm{O}^{3}\right)$ in der Luft. Wie sich diese Faktoren über das Maß ihrer natürlichen jahreszeitlichen Schwankungen hinaus verändern, kann die Biomasseproduktion von KUPs maßgeblich beeinflussen (für weiterführende Analysen siehe Rock et al. (2009). Eine der größten Gefahren stellt dabei die Trockenheit in den Sommerhalbjahren dar, die speziell zum Zeitpunkt der Begründung einer neuen KUP sehr negative Folgen haben kann. Im Gegensatz dazu können auch zeitlich und örtlich begrenzte Starkregenereignisse Probleme mit der Befahrbarkeit der Flächen hervorrufen. 
Die Effekte der langsam steigenden Konzentration an $\mathrm{CO}^{2}$ und $\mathrm{O}^{3}$ heben sich vermutlich gegenseitig auf, wobei der Düngeeffekt durch den erhöhten $\mathrm{CO}^{2}$-Eintrag als positiv bewertet wird. (Rock et al., 2009)

Bei einem Vergleich der von Rock et al. (2009) durchgeführten Ertragssimulationen mittels verschiedener Klimamodelle zeigt sich ein deutlich stärkerer Einfluss der Standorteigenschaften bei ertragsschwachen Standorten als durch die zu erwartenden Klimaänderungen.

\subsubsection{Rechtlicher Rahmen}

KUPs sind im Rahmen des EU-Agrarförderrechtes förderfähig und können als Ökologische Vorrangflächen eingestuft werden (Nitsch et al., 2016) bzw. sind in Form von Kulturlandschaftsprogrammen (KULAP) seitens der Bundesländer anerkennungswürdig.

Laut Bundeswaldgesetz (BWaldG) § 2 Abs. 2 Nr. 1 sind: „Grundflächen, auf denen Baumarten mit dem Ziel baldiger Holzentnahme angepflanzt werden und deren Bestände eine Umtriebszeit von nicht länger als 20 Jahren haben (Kurzumtriebsplantagen), [...]" kein Wald im Sinne des Gesetzes (BMEL, 2017). Da der Status als landwirtschaftliche Fläche erhalten bleibt, ist somit auch keine Aufforstungsgenehmigung nötig (Unseld et al., 2014).

Die naturschutzrechtliche Bewertung von KUPs erfolgt auf der Grundlage des BNatSchG und auf den gesetzlichen Regelungen des jeweiligen Bundeslandes. Die Föderalismusreform von 2006 ermöglicht durch die konkurrierende Gesetzgebung den Bundesländern ein Abweichungsrecht (Art. 74 Abs. 1 Nr. 29 Grundgesetz (GG) in den Bereichen des Naturschutzes und der Landschaftspflege. Somit verfügen manche Bundesländer über eigene Landesnaturschutzgesetze (bspw. Bayern, Bremen, usw.) oder Ausführungsgesetze zum BNatSchG (bspw. Hamburg, Niedersachsen, usw.). (DB, 2017)

Zur rechtlichen Einordnung von KUPs und deren Gehölze als Dauerkulturen, führen Böhm et al. (2017) folgendes aus: „Dauerkultur: Hierunter fallen alle Gehölze, die als „Niederwald im Kurzumtrieb“ [DirektZahIDurchfV, 2020] angebaut wurden. Hierzu gehören beispielsweise die Gehölze in Kurzumtriebsplantagen, [...]. Damit Gehölze als Dauerkultur anerkannt werden können, müssen verschiedene Rahmenbedingungen eingehalten werden. Hierzu zählen eine maximale Umtriebszeit von 20 Jahren, eine Mindestflächengröße von 0,3 ha (da jede Gehölz-Dauerkulturfläche einen eigenständigen Ackerschlag darstellen muss und für jenen in den meisten Bundesländern diese Mindestgröße gilt) und die Beschränkung auf bestimmte, für den Kurzumtrieb geeignete Baumarten (mehrere Baumarten auf einer Fläche sind nicht zulässig). Gehölze mit dem Status einer Dauerkultur sind beihilfefähig, d.h. ihre komplette Fläche wird als Teil der landwirtschaftlichen Nutzfläche anerkannt und ist daher basisprämienberechtigt." 


\subsubsection{Etablierung einer Kurzumtriebsplantage}

\subsubsection{Planung}

\subsection{Anlage}

Die Anlage einer KUP erfordert eine detaillierte Planung, bei der nach Möglichkeit alle standörtlichen, baumphysiologischen und sonstigen Rahmenbedingungen berücksichtigt werden, um die Ziele des Bewirtschafters zu erreichen.

Vorab sollte die gesamte Erntelogistik in den Aufbau miteingeplant und somit Aspekte wie ganzjährige Befahrbarkeit, Hangneigung und Lagerplätze berücksichtigt werden.

Abhängig von dem Zielprodukt (Stammholz, Industrieholz, Energieholz) haben sich die beiden Pflanzsysteme der Einzel- bzw. Doppelreihe etabliert. Hierbei schwanken die Pflanzdichten von 200 - 11.000 Pflanzen / ha bei Pflanzverbänden zwischen $7 \times 7 \mathrm{~m}$ (als Einzelreihe) und 0,75 / 1,6 x 0,8 m (als Doppelreihe). Eine vollflächige Bodenbearbeitung wie bspw. Pflügen im Herbst und Feingrubbern im Frühjahr ist gebräuchlich. (Schildbach et al., 2009)

\subsection{Pflanzung und Pflege}

Das zeitige Frühjahr (März bis Mitte Mai) ist der bevorzugte Zeitpunkt für eine möglichst sorgsame Pflanzung des qualitativ hochwertigen Pflanzmaterials. Frühjahrstrockenheit kann dadurch leichter überwunden werden und die Pflanzen haben die gesamte Vegetationsperiode über Zeit zur Etablierung. Eine Herbstpflanzung ist auch möglich und wird bevorzugt mit bewurzelten Steckhölzern bzw. wurzelnackten Pflanzen durchgeführt.

Die manuelle Pflanzung eignet sich bei Flächengrößen bis drei Hektar. Sie ist meist aufwendiger, jedoch können alle Pflanzmaterialtypen verwendet werden. Die maschinelle Pflanzung bietet den Vorteil, dass hierbei modifizierte land- oder forstwirtschaftliche Pflanzmaschinen eingesetzt werden können.

Besondere Pflege ist in den ersten zwei Wuchsjahren bis zur Etablierung der Plantage nötig. Mögliche Gefahren bei denen ein aktives Eingreifen nötig wird, sind hierbei bspw. langanhaltende Trockenheit, die eine künstliche Beregnung erforderlich machen können, oder ggf. Pflegemaßnahmen zur Regulierung des Begleitwuchses.

$\mathrm{Da}$ es sich bei frischangelegten KUPs um ein für Rehwild attraktives Äsungsangebot handelt und auch in späteren Jahren Fege- und Schälschäden hohen wirtschaftlichen Schaden verursachen können, ist ein aktives Wildmanagement bzw. eine Zäunung notwendig. (Schildbach et al., 2009)

\subsubsection{Standort- und Baumartenwahl}

Im Allgemeinen sind für die Wahl der Baumart bei KUPs andere Anforderungen zu stellen als für Waldbäume. Zwar sind beispielsweise Eigenschaften wie gleichmäßiger Jahrringaufbau, Feinastigkeit, Geradschaftigkeit, usw. für die Qualitätsansprache für Holz aus Wäldern wichtig, aber sie sind, abhängig von den Bewirtschaftungszielen, für KUPs teilweise belanglos. 


\subsection{Allgemeine Anforderungen an Baumarten}

Wichtige Eigenschaften für KUP-Baumarten sind in Ergänzung zu Schildbach et al. (2009) und Hofheinz (2016):

- Einfache Vermehrbarkeit und hohe Anwuchssicherheit: Speziell in den durch den Klimawandel häufiger zu erwartenden Trockenjahren ist dies von größer werdender Bedeutung.

- Jugenddichtstandsverträglichkeit: Dadurch wird eine hohe Biomasseproduktion bei vergleichsweise kurzer Umtriebszeit begünstigt.

- Rasches Jugendwachstum: Dies ist gewöhnlich bei Lichtbaumarten mit Pioniercharakter zu finden, deren Hauptwachstumsphase, durch den frühen Kulminationspunkt des laufenden Zuwachses, eine kurze Umtriebszeit ermöglichen.

- Hohe Biomasseleistung: Diese ist ausschlaggebend für die Wirtschaftlichkeit einer KUP, wobei Qualitätsansprüche wie sie bei Holz aus Wäldern vorherrschen, vernachlässigbar sind.

- Geringe Anfälligkeit für abiotische und biotische Schäden: Da oft nur ein einziger Klon zur Begründung einer kompletten KUP Verwendung findet, muss bereits bei der Züchtung auf hohe Widerstandsfähigkeit geachtet werden.

- Stockausschlagvermögen: Bei kurzen Umtriebszeiten und somit häufigen Ernten, können auf diese Weise Kulturkosten eingespart werden.

Weitere Eigenschaften wie bspw. Toleranz gegenüber Staunässe, Trockenresistenz, usw. können abhängig von den Bewirtschaftungszielen und vor allem dem Standort erforderlich sein.

\subsection{Standortbedingungen und Ziele des Bewirtschafters}

KUPs werden in Deutschland vorwiegend auf mineralischen Standorten ohne Grundwassereinfluss und mit geringem bis mittleren Nährstoffangebot angebaut. Dabei ist die richtige Baumartenwahl entscheidend für den Erfolg der Anlage, da sich die Arten bezüglich ihrer Standortansprüche (Licht, Nährstoffe, Wasser, ...) unterscheiden. (Schildbach et al., 2009)

Hofheinz (2016) ergänzt treffend hierzu: „Aus diesem Grund ist es zunächst wichtig zu klären, welche Bedingungen auf der Fläche vorherrschen, um diese mit den ökologischen Standortsamplituden der in Frage kommenden Alternativen abzugleichen. [...] Abhängig von den Standortfaktoren wie Klima (Temperatur und Niederschlag), Boden, Wasserhaushalt aber auch zum Beispiel Exposition, ist die Baumart zu wählen.“

Die Zielstellung bei der Anlage der KUP ist als zweiter wichtiger Punkt bei der Auswahl der richtigen Baumart zu nennen. Schildbach et al. (2009) unterscheidet hierbei die:

- Produktbezogene Zielstellung: Hierbei werden die Produkte Energieholz (Hackschnitzel) oder Industrie- / Stammholz angestrebt.

- Ökonomische Zielstellung: Dabei sind finanzielle Überlegungen wie z.B. geringe Anlagekosten, ein schneller Kapitalrückfluss (kurze Umtriebszeiten) und eine möglichst hohe Rendite entscheidend.

- Bewirtschaftungsbedingte Zielstellung: Um die Bewirtschaftung einer Fläche zu ändern, bspw. bei zu kleinen oder zu trockenen Standorten, kann die Anlage einer KUP sinnvoll sein. 


\subsection{Paulownia als mögliche KUP-Baumart}

Nehring et al. (2013) stufen P.tomentosa als potenziell invasiv ein (2.1.4 Bewertung der Invasivität von P.tomentosa). Es ist aber davon auszugehen, dass diese frühsukzessionale Art durch ihren hohen Lichtbedarf schnell von später auftretenden, deutlich schattentoleranteren Arten verdrängt wird (Moore and Lacey, 2009; Vor et al., 2015).

Paulownia verfügt über alle relevanten Charaktere einer KUP-Baumart, die für eine mögliche Anbauwürdigkeit sehr vorteilhaft sind (Abb. 8):

- Einfache generative und vegetative Vermehrbarkeit und ein hohes Stockausschlagvermögen (Zhu et al., 1986)

- Hohe Trockentoleranz nach erfolgreichem Anwuchs (Cabi, 2019)

- Sehr starkes Jugendwachstum mit frühem Kulminationspunkt (Stimm et al., 2013)

- Sehr hohe Biomasseleistung (Cabi, 2019; Zhu et al., 1986)

als eher nachteilig zu erwähnen sind:

- Nur geringe Jugenddichtstandsverträglichkeit: folglich sind große Pflanzverbände oder frühzeitige Durchforstungen nötig (Cathaia, 2020; WeGrow, 2020)

- Anfälligkeit gegenüber einer Vielzahl von biotischen Schäden ist vorhanden, bedarf aber der weiteren Erforschung (Hao et al., 2004)

Eine privatrechtliche Klage zur Aufnahme von P.tomentosa in die Liste der KUPBaumarten wurde seitens des Verwaltungsgerichtes Köln im Jahr 2014 abgelehnt (VwG Köln, 2014). Ein Erfolg dieser Klage wäre die nötige Voraussetzung gewesen, um eine finanzielle Förderung für diese Baumart im Rahmen von landwirtschaftlichen Dauerkulturen zu er-halten.

Trotz dessen wird diese Baumart in vielen Teilen Deutschlands auf landwirtschaftlichen Flächen, sowohl einzelbaumweise als auch in Plantagen großflächig schon seit Jahren angebaut (siehe hierzu Cathaia, 2020; WeGrow,

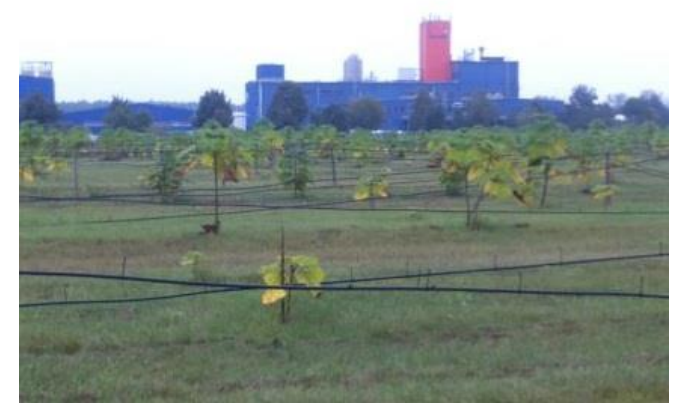

Abb. 8: Paulownia-KUP mit künstlicher Bewässerung (schwarze Linien), bei der Stadt Premnitz, Land Brandenburg. 2020).

\subsubsection{Abiotische und biotische Risikofaktoren}

Die wirtschaftlichen Aspekte zur leistungsorientierten Produktion von Dendromasse sind maßgebend bei der Anlage einer KUP und können doch stark durch das mögliche Auftreten von Schadfaktoren beeinflusst werden. Die vergleichsweise kurze Umtriebszeit kann sich positiv auf die negativen Effekte einiger Schadfaktoren auswirken, gleichzeitig verkürzt sich aber der Kompensationszeitraum, um mögliche Verluste bei der Ausbildung der Dendromasse auszugleichen. 
Risikofaktoren beeinflussen sich sowohl positiv als auch negativ gegenseitig und erst ihr kombiniertes Auftreten birgt das größte Risiko für den wirtschaftlichen Erfolg. Oder wie es Helbig \& Müller (2009) beschreiben: „Je weiter die Standortsbedingungen vom Optimum einer Sorte oder Baumart entfernt sind, desto höher ist die Wahrscheinlichkeit für ein Auftreten von Schäden und Schaderregern und desto geringer ist die Fähigkeit der Pflanze, diese abzuwehren oder zu tolerieren. Des Weiteren hat die Umgebung einer Plantage große Bedeutung als Ursprungsort von Schaderregern." (Helbig and Müller, 2009)

\subsection{Abiotische Risiken}

Die Möglichkeiten zur Beeinflussung von abiotischen Risiken sind nur gering, und sie entwickeln sich erst zu einem Schaden, wenn sie in extremer Weise auftreten.

Durch den schneller voranschreitenden Klimawandel ist mit dem häufigeren Auftreten von Trockenmonaten bzw. -jahren zu rechnen. Künstliche Bewässerungsmaßnahmen im ersten Jahr der Anlage einer KUP können für Abhilfe sorgen und die Pflanzen, aufgrund ihres besser ausgeprägten Wurzelsystems, für zukünftige Trockenperioden stärken. Auch sollte der Anlagezeitpunkt möglichst früh im Jahr erfolgen, auf dass die Pflanzen noch vor der möglichen Frühjahrstrockenheit erste Wurzeln ausbilden können. Frostschäden entstehen auf KUPs meist durch Spätfröste von April bis Mai oder durch zu milde Winter und dem dadurch verfrühten Austrieb der Pflanzen. Die finanziellen Schäden können sehr hoch sein und laut Friedrich (1999) bspw. bis zu 50 \% Ausfälle bzw. Leistungseinbußen beim Zuwachs von Pappeln erreichen.

Durch die Standortwahl bzw. dem Anbau von Hecken und Windschutzstreifen oder die aktive Randgestaltung kann der negative Einfluss von Wind auf eine Plantage stark minimiert werden. (Helbig and Müller, 2009)

\subsection{Biotische Risiken}

Da KUPs von einer geringen genetischen Diversität und räumliche Homogenität geprägt sind, besteht eine hohe Wahrscheinlichkeit von Schäden durch Pilze, Bakterien, Viren, Insektenmassenvermehrungen, usw.

Frühzeitige Versäumnisse bzw. Mängel bei der Planung der Plantage erhöhen das Risiko biotischer Schäden, die sich auch nur bedingt mit Gegenmaßnahmen wie bspw. Pflanzenschutzmittel ausgleichen lassen und dabei noch erhebliche Mehrkosten verursachen. (Helbig and Müller, 2009)

In den ersten Jahren nach Anlage der KUP kann Begleitvegetation eine so starke Beschattung erzeugen, dass es bei Lichtbaumarten zu Wachstumsverzögerungen bzw. Ausfällen kommen kann und zur Vermeidung eine mechanische oder chemische (Herbizide) Begleitwuchsregulierung nötig wird. Clay \& Dixon (1997) berichten von Untersuchungen an verunkrauteten Pappeln und Weiden die ein um $60-90 \%$ geringeres Triebgewicht als unkrautfreie Vergleichspflanzen aufwiesen.

Auch lässt sich durch die Vermeidung einer flächigen Bodenvegetation die Ausbildung von größeren Mäusepopulationen und mit ihr die Schäden durch Benagen bzw. Wurzelfraß verringern. 
Um das Auftreten von Krankheiten durch Bakterien, Pilze und Viren zu minimieren bzw. ihre Entwicklung und Ausbreitung zu verlangsamen, empfiehlt sich der Anbau mehrerer Baumarten bzw. Sorten auf einer Plantagenfläche. Nach Ansicht von McCracken \& Dawson (1997) sind dazu mindestens drei verschiedene Sorten mit unterschiedlichen Anfälligkeiten notwendig.

Sortenmischungen in Kombination mit einem standortangepasstem Flächendesign bieten nach Helbig \& Müller (2009) auch Möglichkeiten zur vorbeugenden Schädlingskontrolle. Generell ermöglichen KUPs gute Entwicklungsbedingungen für Schadinsekten aufgrund ihres hohen Anteils an nährstoffreicher Blattmasse (Helbig and Müller, 2008). Darüber hinaus bieten sie laut Bergström \& Guillet (2002) hochwertige Äsung für Wild und sind daher besonders verbissgefährdet, was zur Verbuschung bzw. zum Absterben von Pflanzen führen kann (Friedrich, 1999). Auch können Fege- bzw. Schälschäden bei hoher Wilddichte lokal starke Schäden hervorrufen. Kleinere KUPs, um zwei Hektar, sollten daher immer gezäunt werden, was relativ kostenintensiv ist im Vergleich zu großflächigen KUP-Anbauten, auf denen sich der Wildeinfluss zumeist verringert. (Helbig and Müller, 2009)

\subsubsection{Bewirtschaftung}

\subsubsection{Bewirtschaftungsstrategien}

Die vorab festgelegte Anbau- und Nutzungsstrategie bestimmt direkt die Bewirtschaftungsstrategie. Neben der stofflichen ist auch eine energetische Nutzung der Endprodukte möglich, wobei es abhängig von der Marktentwicklung zu einer Verschiebung von Nutzungspräferenzen kommen kann. (Gerold et al., 2009)

\subsection{Stoffliche Nutzung}

Die stoffliche Nutzung dient der „[...] Bereitstellung von Faserrohstoff für die Papierherstellung und von Material für die Span- und Faserplattenindustrie" (Gerold et al., 2009). Für die Anlage einer solchen KUP wird nur eine geringe Pflanzenanzahl benötigt (1.000 - 2.000 Pflanzen / ha), was die Kosten deutlich senkt. Des Weiteren kann für die Ernte auf typische land- bzw. forstwirtschaftliche Holzernteverfahren zurückgegriffen werden. Allerdings ist die lange Kapitalbindung aufgrund der hohen Umtriebszeit (meist 15 - 20 Jahre) nachteilig. Auch ist die Biomasseleistung im Vergleich zur energetischen Nutzung mit durchschnittlich ca. 9 tatro ha $^{-1} \mathrm{a}^{-1}$ geringer $^{14}$. (Gerold et al., 2009)

\subsection{Energetische Nutzung}

Das Ziel der energetischen Nutzung einer KUP ist eine möglichst hohe Biomasseproduktion, die laut Boelcke (2007) auf schlechten Standorten bei unter 10 tatro ha $\mathrm{ha}^{-1} \mathrm{a}^{-1}$ liegen kann und auf guten Standorten über $20 \mathrm{tatro}_{\text {ha- }} \mathrm{ha}^{-1}$ erreicht.

Gerold et al. (2009) geht daher von einem mittleren Ertrag in Höhe von 8 bis 12 tatro ha $^{-1}$ $\mathrm{a}^{-1}$ aus. Somit sind klassische Qualitätsmerkmale wie bspw. Feinastigkeit, Geradschaftigkeit, usw. die bei der stofflichen Nutzung bedeutsam sein können, bei der energetischen Nutzung vernachlässigbar.

14 tatro ha-1 $\mathrm{a}^{-1}=$ Tonne atro (= Maßeinheit für eine Tonne absolut trockenen Holzes) pro Hektar und pro Jahr. 
Zwar werden deutlich höhere Pflanzenzahlen benötigt (8.000 - 10.000 Pflanzen / ha), was die Anlagekosten der KUP erhöht, jedoch ist durch die geringere Umtriebszeit (alle 2 bis 5 Jahre) ein schneller Kapitalrückfluss möglich. Nachteilig ist hingegen die zusätzliche, nicht land- bzw. forstwirtschaftlich typische Technik die zumeist für die Ernte gebraucht wird und zusätzliche Kosten verursacht (Scholz et al., 2004). (Gerold et al., 2009)

\subsection{Weitere Nutzungen}

Natürlich sind auch weiteren Nutzungen bzw. andere Erzeugnisse denkbar, wobei diese zumeist als Nischenprodukte gelten. Beispielsweise lässt sich mit dem Anbau von Weiden Salicin gewinnen, und die Ruten können zusätzlich für den Landschaftsbau verwendet werden. (Gerold et al., 2009)

Auch ermöglicht bspw. der Anbau von Robinie mit mittelfristigem Umtrieb die Produktion von Zaunpfahlsortimenten (Landgraf et al., 2005).

\subsubsection{Wirtschaftlichkeit}

Der Aufbau einer KUP unterscheidet sich im Vergleich zu klassischen landwirtschaftlichen Kulturen insofern, dass für eine KUP hohe Investitionen bei der Begründung entstehen. Hinzu kommt ein langer Produktionszeitraum, der durchweg von unregelmäßigen Zahlungsströmen geprägt ist.

Die Einschätzung, inwiefern eine KUP finanziell mit dem Anbau von Ackerfrüchten konkurrieren kann, ist insofern schwierig, als dass die Agrarpreise einer zunehmenden Volatilität unterliegen.

Allerdings verfügen viele landwirtschaftliche Betriebe über Flächen, die nur bedingt zur intensiven ackerbaulichen Nutzung geeignet sind und auf denen der Aufbau einer KUP möglich und sinnvoll wäre. Außerdem kann die Bewirtschaftung einer KUP hilfreich sein, die über das Jahr entstehenden Arbeitsspitzen des Landwirtschaftsbetriebes abzubauen. (Wagner et al., 2009)

\subsection{Ernte}

Da die Erntetechnologie maßgeblich die Produktionskosten mitbestimmt, sollte sie noch vor der ersten Anpflanzung geplant werden, „da deren Wahl unter anderem von Sorte, Rotationsintervall, Reihen- und Pflanzabstand abhängig ist" (Scholz \& Lücke, 2007). Grundsätzlich lassen sich die Ernteverfahren nach Scholz \& Lücke (2007) in die folgenden drei Bereiche unterscheiden:

- Stammholzlinien: stoffliche Nutzung zur Produktion von Stammabschnitten

- Bündellinien: energetische Nutzung zur Produktion von lose oder gebundenen Bündeln

- Hackgutlinien: energetische Nutzung zur Produktion von Hackschnitzeln

Nach der letzten Ernte erfolgt zumeist eine Flächenrückwandlung. Dabei wird die mit Bäumen bepflanzte Fläche durch Rodetechnik (z.B. Rodefräsen) vollständig in konventionelles Ackerland umgewandelt. Die Ausbildung von neuen Trieben, aus den noch im Erdreich verbliebenen Wurzelstücken, kann durch weitere Bodenbearbeitungsmaßnahmen bzw. der Einsaat einer Folgekultur unterdrückt werden. (Scholz et al., 2009) 


\subsubsection{Naturschutzfachliche Betrachtungen}

\subsubsection{Vergleich zu Wäldern}

2.2.4.1.1 Natürlichkeitsgrad versus Bewirtschaftungsintensität KUPs können bei einem Vergleich von Gehölzökosystemen ${ }^{15}$ (Abb. 9) als Gehölzbestände mit partieller Segregation (teilweiser Entmischung) beschrieben werden. Aufgrund des vorrangigen Ziels, der Holzproduktion, kann ihr Natürlichkeitsgrad als gering angesehen werden, wobei die Intensität der Bewirtschaftung vergleichsweise hoch ausfällt. Neuwald ist beispielsweise auf Acker- bzw. Bergbau-flächen standörtlich mit einer KUP durchaus vergleichbar und auch vom Natürlichkeitsgrad anfänglich sehr ähnlich, aber die Bewirtschaftungs-intensität fällt, im Laufe der Entwicklung hin zu einem Wirtschaftswald, deutlich geringer aus.

\subsection{Vergleich mit Natur- bzw. Kulturwäldern}

Bei einem Vergleich von KUPs mit Natur- bzw. Kulturwäldern, wie er von Schmidt \& Gerold (2008) vorgenommen wird, zeigt sich eine Unvereinbarkeit mit den Zielen des Naturschutzes wie z.B. nach mehr Naturnähe in Kulturlandschaften oder der Förderung der Biodiversität, usw.

KUPs sind laut Schmidt \& Glaser (2009): „künstlich begründete Bestände, überwiegend [...] Reinbestände aus ausschlagfähigen, nichtheimischen oder gebietsfremden Gehölzsippen (Arten, Hybriden) oder ausgewählten Sorten bzw. Klonen. Damit entstehen neuartige Ökosysteme in der Landschaft mit Eigenschaften, die unvereinbar mit Naturschutz erscheinen [...]". Da KUPs aber nicht in Wäldern angelegt werden, ist ein solcher Vergleich weniger hilfreich, und es sollten eher Agrarlandschaften und deren Biotope als Referenzsysteme

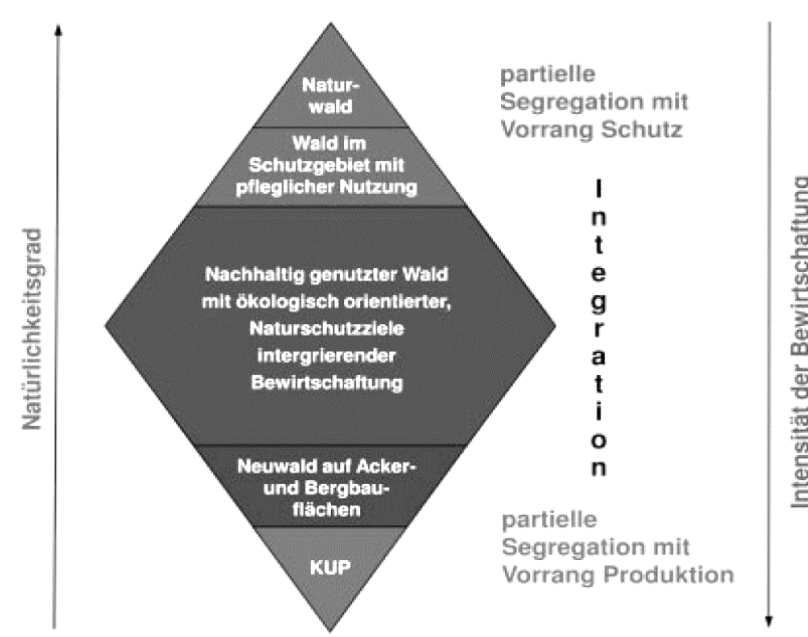

Abb. 9: Vergleich der Bewirtschaftungsintensität und des Natürlichkeitsgrades von Gehölzökosystemen, nach Schmidt \& Gerold (2008) übernommen und verändert aus Schmidt \& Glaser (2009). dienen.

\footnotetext{
${ }^{15}$ Integration = ökologisch orientieret bzw. naturnahe Waldbewirtschaftung auf dem überwiegenden Teil der Waldfläche; partielle Segregation von Flächen (mit Vorrang Schutz), auf denen die Ökosysteme einer Selbstregulation überlassen bleiben und dementsprechend auf die Bewirtschaftung verzichtet wird (Naturwaldregeneration in Schutzgebieten nach Naturschutz- und Forstrecht), partielle Segregation von Flächen mit Vorrang Holzerzeugung durch intensiveres Management (KUP) auf Ackerland. Nach Schmidt \& Gerold (2008), übernommen und verändert aus Schmidt \& Gläser (2009).
} 


\subsubsection{Vergleich zu landwirtschaftlichen Kulturen}

Der Aufwand zur Bewirtschaftung einer KUP liegt vergleichsweise unter dem von einbzw. mehrjährigen Ackerkulturen (Energie-, Futter-, Nahrungspflanzen) und erlaubt daher aus landschaftsökologischer Sicht eine positivere Bewertung.

Natürlich bedarf es zu dieser Einschätzung einer näheren Betrachtung der unterschiedlichen Phasen im Gesamtsystem der KUP (wie Begründung, Bestandesschluss, Ernte, usw.) im Vergleich zu den Biotop- und Habitat-Bedingungen von landwirtschaftlichen Kulturen. (Schmidt \& Glaser, 2009)

\subsection{Beeinflussung des Wasserhaushaltes}

Die Witterung und Wasserverfügbarkeit innerhalb einer Vegetationsperiode steuert indirekt die Transpirationsrate einer KUP. Die angebauten Baumarten bestimmen durch ihre Kronenarchitektur (Abfluss an Stamm und Ästen) und den Blattflächenindex die Höhe der Interzeptionsverluste. Petzold et al. (2009) gehen davon aus, dass bspw. durch Pappel und Weiden tendenziell mehr Wasser auf dem gleichen Standort verbraucht wird, als durch den vergleichbaren Anbau von landwirtschaftlichen Kulturen. Die jährliche Sickerwasserrate verringert sich (Murach et al., 2007), was zu Problemen bei der Grundwasserneubildung führen kann (Wahren et al., 2007). Allerdings verringert sich durch den verminderten Oberflächenabfluss als Gegeneffekt dazu der Bodenabtrag (Erosion).

KUPs als Form der Landnutzungsänderung besitzen „[...] das Potential, bei kleinen bis mittelgroßen Regenereignissen die Scheitelwellen des Abflusses zu verzögern“ was zum Hochwasserschutz beiträgt (Petzold et al., 2009). Auch der Bodeneintrag in Talsperren und die dadurch hervorgerufene Sedimentbildung verringert sich.

\subsection{Standortwahl und Rohstoffquelle}

Bei der Auswahl des geeigneten Standortes einer KUP kann es zu hohem Konfliktpotenzial zwischen den Zielen des Naturschutzes und den Interessen des Bewirtschafters kommen. Gleichzeitig kann aber die biologische Vielfalt, unter Beachtung von regionalen Leitbildern und Naturschutzzielen, durch eine gezielte nachvollziehbare Standortwahl, Anlage und Nutzung einer KUP gefördert werden. Abhängig von der Eigenart und Schönheit einer Landschaft lässt sich diese mit den räumlichen Strukturen einer KUP bereichern und die biologische Vielfalt fördern. (Schmidt and Glaser, 2009)

Wenn der auf KUPs zyklisch nachwachsende Rohstoff Holz als alternative Energiequelle verwendet wird, kann dies zur Substitution von fossilen Energieträgern beitragen und somit dem Klimaschutz dienlich sein. Außerdem wäre diese Biomasse vom Acker eine indirekte aber doch „[...] wichtige Ergänzung der Holzpotenziale aus dem Wald [...]" (Schmidt and Glaser, 2009). 


\subsection{Agroforst}

Agroforstsysteme finden sich schon seit Jahrhunderten in der deutschen Landschaft, und sie sind in Form von Heckenlandschaften, Streuobstwiesen und Waldweiden selbst heute noch leicht erkennbar (Eichhorn et al., 2006; Herzog, 1997).

Ausgelöst durch die Industrialisierung kam es im ausgehenden 19. und besonders zu Beginn des 20. Jahrhunderts verstärkt zu einer Spezialisierung in der Landwirtschaft und somit zu einer Trennung in der Nutzung, die ursprünglich auf ein und derselben Fläche stattgefunden hat. Dies hatte einen hohen Verlust von AFS zu Folge und erst in jüngerer Zeit werden AFS langsam wiederentdeckt und helfen die traditionelle Trennung von Land- und Forstwirtschaft zu überwinden.

\subsubsection{Historische Landnutzungssysteme}

\subsubsection{Ein Blick in die historische Entwicklung von AFS}

In der Historie findet sich eine Vielzahl von AFS, mit unterschiedlich intensiver Nutzung, die eine sehr mannigfache Anordnung von Bäumen bzw. Gehölzen aller Art zur Folge hatten. Die Trennung von Land- und Forstwirtschaft hat zu einem großräumigen Verlust dieser Nutzungssysteme geführt. (Konold and Reeg, 2009)

\subsection{Historische Systeme}

Eines der ältesten und noch bis in die heutige Zeit sporadisch genutztes AFS ist die Schneitelwirtschaft. Vermutlich hat der Mensch nicht erst seit dem Neolithikum (Jungsteinzeit), mit dem Beginn des Anbaus von Kulturpflanzen und der Haltung von Haustieren, aktiv in die Landschaft eingegriffen. Doch die Gewinnung von Laub (Schneiteln) zum Zweck der Viehfütterung führte zu einer großflächigen Veränderung des Lebensraumes und nebenbei entstanden bizarre Baumgestalten, die die Sagen- und Mythenwelt der Menschen anregten. In Mitteleuropa wurden häufig Esche, Ulme, Bergund Feldahorn zum Schneiteln verwendet.

Eine weitere und heute fast vergessenes AFS ist das Zeidelwesen bzw. die Zeidlerei, womit die Gewinnung von Wildhonig seit dem 13. Jahrhundert bezeichnet wird. Dafür wurden in sogenannten Beutekiefern Höhlen gehauen, um Bienenvölkern Wohnraum zu bieten. Neben Kiefern wurden selten auch Fichten, Linden, Eichen und Pappeln verwendet. Um die Beutekiefern herum wurde die Heide bewirtschaftet, wodurch sich eine weitestgehend offene, sehr großräumige Landschaft herausbildete.

Die sogenannten Holzwiesen sind eine weitere altertümliche Verbindung von aktiv gepflegten Baumbeständen zur Holzgewinnung bei gleichzeitiger Nutzung der Wiesen für Futter in Form von Heu. Abhängig von den Standorten fanden dabei Baumarten wie Buche, Esche, Eberesche, Eiche, Fichte, Hasel, Bergahorn, Kiefer und Birke Verwendung. Konold \& Reeg (2009) zitieren hierbei Gradmann (1950) mit den Worten: „So sind Parklandschaften entstanden, an denen sich der Landschaftsgärtner ein Muster nehmen kann". Diese Aussage unterstreicht wie früher schon geometrische Formen von AFS das Landschaftsbild prägten und z.B. in späteren Stadtgärten zur Nachahmung anregten. (Konold and Reeg, 2009) 


\subsection{Historische und bis heute genutzte Systeme}

Weitere historische AFS sind z.B. die Kopfweidenkultur bzw. Kopfholzwirtschaft die vielfach der Schneitelwirtschaft gleicht und wohl in ihrer Urform schon bei den Römern Verwendung fand. Dabei wurden Weidenbäume in wiederkehrender Nutzungsperiode von meist drei bis sechs Jahren "geköpft" und die dabei gewonnen Weidenruten bspw. als Flechtmaterial für Weidenfaschinen, die den Wasserbau noch bis ins 19. Jahrhundert dienten, verwendet.

Die noch heute am häufigsten anzutreffende Form einer AFS ist die der Obstkultur. Dabei hatte der Obstbau mit Hochstämmen gerade auch in Klöstern zunehmend wichtige gestalterische Aufgaben und mit seinen vielen geometrischen Formen zur Ordnung der Landschaft, die auch noch heute vielerorts erkennbar ist, beigetragen.

Im Gesamtbild betrachtet, verwundert es also nicht, dass sich der königlich sächsische Oberforstrat Heinrich Cotta (Cotta (1819), zitiert nach Konold \& Reeg, 2009) in seinem Buch „Die Baumfeldwirtschaft“ sehr ausführlich mit AFS auseinandersetzt und dabei zu dem Schluss kommt: dass in ordentlichen Waldungen das Einzelne sich dem Ganzen unterordnen müsse und so ,jede einzelne Stelle des Bodens auf die angemessenste Weise" zu nutzen sei. Auf diese Weise könne man „dem kleinsten Raume [...] die Holzart geben, die für inn passt“ und somit „vielerlei Holzarten nebeneinander" erziehen.

Schon sehr früh wurde also erkannt, wie sich das Nützliche mit dem Schönen verbinden lässt und eine Vielzahl der damaligen Argumente sind noch heute gültig und werden wieder wichtiger und regen so zur Pflanzung von Bäumen außerhalb des typischen Waldes an. (Konold and Reeg, 2009)

\subsubsection{Definition und Rechtliche Rahmenbedingungen}

\subsubsection{Multifunktionalität von Agroforstsystemen}

Agroforstsysteme haben durch ihre Multifunktionalität das Potenzial heutige Landnutzungsansprüche sowohl in ökonomischer als auch ökologischer Hinsicht zu befriedigen. Dabei erweitern sie das landwirtschaftliche Produktspektrum durch hochwertige Holzsortimente und entfalten zugleich positive Wirkungen auf die sie umgebende abiotische und biotische Umwelt.

Grünewald \& Reeg (2009) untermauern diese Aussagen, mit einer Vielzahl von Literatur, indem sie die folgenden positiven Auswirkungen von Agroforstsystemen auflisten:

- Höhere und nachhaltig gesicherte Erträge

- Höhere Biodiversität

- Verbessertes Mikroklima

- Schutz vor Erosion

- Nährstoffumverteilung aus tieferen Bodenschichten mittels der Blattstreu

- Verminderung von Krankheiten, Schädlingen und klimatischem Stress

- Positive Effekte auf Weidetiere

\subsection{Definition von Agroforst}

Unter Agroforst bzw. Agroforstwirtschaft wird heutzutage eine Form der Landnutzung verstanden: „[...] bei der der Anbau mehrjähriger verholzender Pflanzen mit dem Anbau von annuellen Pflanzen oder der Nutzung von Grünland auf derselben Fläche kombiniert wird. 
Durch die gezielte Wahl der räumlichen Anordnung und zeitlichen Abfolge der unterschiedlichen Elemente [...] können positive ökonomische und ökologische Wechselwirkungen zwischen Bäumen und Sträuchern einerseits und landwirtschaftlichen Kulturen und Nutztieren andererseits gefördert werden" (Grünewald and Reeg, 2009).

\subsection{Produktionsformen}

Bei der Agroforstwirtschaft werden grundlegend zwei Produktionsformen unterschieden. Die Wertholzproduktion dient der Erzeugung qualitativ hochwertiger Holzsortimente, bei der vereinzelt Bäume, bei Umtriebszeiten von ca. 50 - 70 Jahren, auf landwirtschaftlichen Flächen gepflanzt werden. (Grünewald and Reeg, 2009)

Bevorzugt werden dabei lichtbedürftige Arten, die in Wäldern selten bzw. nicht mit ausreichender Qualität vorkommen. Von Vorteil sind dabei die nur geringen Investitionskosten, der zeitlich begrenzte Pflegeaufwand und die dabei leicht zu erzielenden hohen Durchmesserzuwächse. (Brix et al., 2009)

Bei der Energieholzproduktion hingegen werden schnellwachsende Bäume, mit einer Umtriebszeit von ca. 10 Jahren, in dem sogenannten Alley-Cropping-System streifenweise mit Ackerkulturen dazwischen angebaut, was eine Ergänzung zu der vorab behandelten Kurzumtriebsplantage darstellt (Grünewald and Reeg, 2009).

Bei der Kombination beider Produktionsformen wird der Ackerstreifen, mittels schnellwachsender Baumarten, zur Biomasseproduktion verwendet und im AlleyCroping-System werden streifenweise Bäume zur Wertholzproduktion gepflanzt (Grünewald and Reeg, 2009).

Dieses zweihiebige System, mit zwei nach Höhe und Alter getrennten Bestandesschichten, erfordert mehr waldbauliche Kenntnisse als für die Anlage einer KUP. Der spätere Wuchsraum für die Werthölzer muss schon bei der Planung miteinkalkuliert werden. Messungen auf Versuchsflächen haben gezeigt, dass die Werthölzer den Lichteinfall für die schnellwachsenden Hölzer nicht reduzieren. Allerdings kann dieses Anbausystem nur auf eher schlechten Standorten seine betriebswirtschaftlichen Vorteile gegenüber reinen KUP ausspielen. (Unseld, 2009)

\subsubsection{Rechtlicher Rahmen}

\subsection{Rechtlicher Rahmen - früher}

Agroforstsysteme sind seit vielen Jahrhunderten Teil der Landschaft und ihre Verbreitung hing immer wieder an dem zu der Zeit gültigen Rechtsrahmen, wovon eine Vielzahl überlieferter Belege künden.

Beispielsweise wurde bereits im 10. Jahrhundert die Waldweide per Erlass erlaubt und auch wieder untersagt (Dirlmeier, 1982). Im 16. Jahrhundert wurde seitens des Adels der Anbau von Obstbäumen auf Wiesen und Äckern gefördert, um die Ernährung der Untertanen zu verbessern. Aus dem gleichem Grunde wurde die Streuobstwiese das am häufigsten verbreitete AFS im 18. und 19. Jahrhundert (Rösler, 2003). 
Doch von 1951 bis 2000 kam es zu einem mehr als 70 \% Rückgang der deutschen Streuobstbestände (BLfL, 2003). Die Intensivierung und die damit einhergehende Mechanisierung der deutschen Landwirtschaft seit dem Zweiten Weltkrieg hat zu einer Fixierung auf den Ertrag pro Hektar geführt (Gordon et al., 2018) und die räumliche Trennung von forstlicher und landwirtschaftlicher Fläche gefördert (Stuber and Bürgi, 2002). Dieser Trend wurde seitens der EU, mittels einer Rodungsprämie für hochstämmige Obstbäume, von 1965 bis 1974 unterstützt (Herzog, 1998).

\subsection{Rechtlicher Rahmen - heute}

Das heutzutage am häufigsten in Deutschland anzutreffende AFS ist die Streuobstwiese (Rösler, 2003), die nach $\S 2$ (1) des Bundeswaldgesetzes vom Waldbegriff ausgenommen ist (BMEL, 2017) und somit nach landwirtschaftlichem Recht behandelt wird. Die Erzeugung von Forstprodukten in Form von Werthölzern oder Kurzumtriebsstreifen auf landwirtschaftlicher Fläche wird gesondert von der klassischen landwirtschaftlichen Produktion auf Bundes- und Länderebene geregelt (Chalmin and Möndel, 2009).

Eine in der Literatur vielfach diskutierte rechtliche Frage behandelt die Produktion von Werthölzern in Agroforstsystemen und ob es sich dabei möglicherweise um Wald handeln könnte. Chalmin \& Möndel (2009) haben zur Klärung die folgende Tabelle mit Argumenten für und gegen eine Zuordnung von Agroforstflächen zu Wald zusammengestellt (Abb. 10):

\begin{tabular}{|c|c|c|}
\hline $\begin{array}{l}\text { Kriterien zur Bestimmung der } \\
\text { Waldeigenschaft }\end{array}$ & Zutreffend? & Kommentar \\
\hline Verwendung von Forstpflanzen & ja und nein & $\begin{array}{l}\text { Es werden Forstpflanzen und veredelte } \\
\text { Obstbäume verwendet. }\end{array}$ \\
\hline $\begin{array}{l}\text { Ausgenommen vom Waldbegriff } \\
\text { sind kleinere Flächen in der Flur } \\
\text { mit einzelnen Baumreihen. }\end{array}$ & ja und nein & $\begin{array}{l}\text { Hier ist die Gesetzgebung nicht präzise } \\
\text { genug, um eine abschließende Antwort } \\
\text { zu ermöglichen. }\end{array}$ \\
\hline Die Holzproduktion überwiegt. & nein & $\begin{array}{l}\text { Die landwirtschaftliche Nutzung } \\
\text { überwiegt deutlich. }\end{array}$ \\
\hline Kronenschluss & nein & $\begin{array}{l}\text { Die Pflanzabstände zwischen den einzel- } \\
\text { nen Werthölzern erlauben keinen } \\
\text { Kronenschluss. Kronenschluss ist zudem } \\
\text { wegen der starken Beschattung nicht im } \\
\text { Interesse der Landwirtschaft. }\end{array}$ \\
\hline Gefahr der Sukzession & nein & $\begin{array}{l}\text { Die Fläche wird, abgesehen von den } \\
\text { Baumstreifen, landwirtschaftlich genutzt. }\end{array}$ \\
\hline
\end{tabular}

Abb. 10: Vergleich von Wald mit Agroforstsystemen zur Produktion von Werthölzern, verändert nach Chalmin \& Möndel (2009).

Die Anlage von AFS betrifft weniger forstrechtliche als vielmehr agrarrechtliche Frageund Problemstellungen, wie z.B. (siehe Zehlius-Eckert, 2018):

- Eine Mindestgröße von 0,3 ha und einer Mindestseitenlänge von $10 \mathrm{~m}$ bei der Anlage von AFS, was hinderlich bei der Etablierung auf kleinen Schlägen ist. 
- Das zur Verfügung stehende Gehölzartenspektrum ist mittels einer Liste eingeschränkt, was die mögliche Vielfalt beim Anbau verringert.

- Aktuell gibt es in Deutschland keine Förderung von AFS was auch einer fehlenden Umsetzung im GAK-Rahmenplan ${ }^{16}$ geschuldet ist.

- $\quad$ AFS können nicht als Greening-Maßnahme anerkannt werden, da es für sie keine Förderung gibt.

- Für AFS gibt es keine eigene Codierung nach europäischen Codiersystem, zur Förderung seitens der EU.

Es gibt eine Fülle von Regelungen, die eine mögliche Etablierung von AFS unnötig erschweren bzw. einschränken. Zahlreiche Initiativen auch auf Länderebene (Brandenburg und Bayern) versuchen diesbezüglich für Abhilfe zu sorgen (Böhm et al., 2017).

Sonstige relevante rechtliche Regelungen sind nach Zehlius-Eckert (2018) bspw.:

- Forstvermehrungsgutgesetz: Sorten müssen vor der Pflanzung den Status "geprüft" erhalten, was zu einem Mangel an Pflanzgut führen kann. Es ist fraglich inwieweit dies zukünftig noch von Belangen ist.

- Nachbarschaftsrecht: Mindestabstände zu Nachbargrundstücken

- Straßenverkehrsordnung: Mindestabstände zu Straßen

Laut Chalmin \& Möndel (2009) kann die Attraktivität von AFS: „[...] für Politik, Gesellschaft und Landwirtschaft gesteigert werden, wenn man regional angepasste Naturschutzleistungen [bspw. extensive Bewirtschaftung von Baumstreifen] definiert und durch deren Honorierung den landwirtschaftlichen Betrieben ein frühzeitigeres Einkommen aus Agroforstbäumen ermöglicht".

Zehlius-Eckert (2018) sieht die aktuellen Rechtsunsicherheiten als kontraproduktiv und hofft auf die durch den Brexit ausgelöste Veränderung in der Agrarförderung und den inn begleitenden rechtlichen Rahmen.

\subsubsection{Etablierung eines Agroforstsystems}

Agroforstsysteme mit Wertholzproduktion erzeugen astfreies Holz (Furnierqualität) mit großem Stammdurchmesser. Chalmin (2009) bezeichnet sie als „lebendige Sparkonten“. Zwar sind Holzpreise marktabhängig doch der Bewirtschafter kann durch die Wahl des Erntezeitpunktes und durch Diversifizierung, also dem Anbau verschiedener Baumarten, darauf reagieren. Die Holzqualität bspw. durch die Astreinheit und die Dimension des Schaftes werden durch die Häufigkeit und Stärke der Pflegeeingriffe und die Umtriebszeit bestimmt. Daraus leitet sich ein grober Produktionsrahmen wie folgt ab:

- Umtriebszeit ca. 50 bis 70 Jahre

- Astfreie Schaftlänge bei ca. 1/3 der Baumhöhe zum Erntezeitpunkt

- $\quad$ BHD von über $55 \mathrm{~cm}$ (Brix et al., 2009)

16 GAK = Gemeinschaftsaufgabe Verbesserung der Agrarstruktur und des Küstenschutzes, ist ein Förderinstrument der BRD zur Unterstützung der Land- und Forstwirtschaft. 


\subsubsection{Planung}

\subsection{Besonderheiten von Agroforstsystemen mit Werthölzern}

Da bei der Pflanzung der Jungbäume weite Abstände eingehalten werden und in den Folgejahren keine sonst im Wald übliche Durchforstung erfolgt, da die Anzahl der Bäume dafür zu gering ist, muss besonderen Wert auf das Pflanzmaterial und die Pflanzung an sich gelegt werden.

Die Ausrichtung und der Abstand der Baumreihen, sowie die Abstände der Bäume zueinander beeinflusst die landwirtschaftlichen Nutzungsmöglichkeiten.

Die Bäume wachsen nahezu ohne Konkurrenz im Kronenbereich auf, weshalb auch keine natürliche Astreinigung erfolgt und eine künstliche Ästung notwendig wird. Durch die ungehinderte Ausdehnung der Baumkonen nach der letzten Ästungsmaßnahme ist mit hohen jährlichen Durchmesserzuwächsen zu rechnen, was erklärt warum der angestrebte Zieldurchmesser deutlicher früher als bei Bäumen in Wäldern erreicht werden kann. (Brix et al., 2009)

\subsection{Flächenauswahl}

Bei der Auswahl der Fläche sollte die langfristige Wirtschaftlichkeit des gesamten Systems beachtet werden. Jede weitere Baumreihe vermindert den Raum für landwirtschaftliche Erzeugnisse und vermindert somit zumindest anfänglich den klassischen jährlichen Ertrag. Daher werden die meisten Betriebe eher landwirtschaftlich ertragsschwache Standorte favorisieren, was jedoch die Wertholzqualität negativ beeinflussen kann. (Chalmin, 2009)

Im Gegensatz dazu kann die Anzahl der Baumreihen auf ertragreichen Standorten verringert werden, was die kurzfristigen landwirtschaftlichen Ertragsverluste verringert. Hinzu kommt noch die Verminderung des Randeffekts der Baumreihenränder, die in Konkurrenz zur landwirtschaftlichen Nutzung stehen können (Palma et al., 2005).

\subsubsection{Anlage}

\subsection{Baumreihen und Baumabstand}

Der Schattenwurf der Baumkronen nimmt mit dem Entwicklungsgrad der Bäume kontinuierlich zu und hat Einfluss auf die unmittelbare Umgebung. Deshalb bietet es sich an, die Baumreihen in Nord-Süd-Ausrichtung anzupflanzen, um beim Wandern der Sonne über den Tag hinweg eine gleichmäßige Verteilung der Schattenbildung zu beiden Seiten der Baumreihe zu erreichen. (Chalmin, 2009)

Die individuellen Standorteigenschaften sollten bei der Festlegung der Reihen- und Baumabstände berücksichtigt werden. Größere Reihenabstände können besonders in trockeneren Gebieten sinnvoll sein, da so der generelle Wasserverbrauch durch die Bäume geringer ausfällt und trotzdem ihr windbremsender und evaporationsverringernder Effekt genutzt werden kann (Young, 1997).

Auf besonders windexponierten und erosionsanfälligen Standorten kann sich durch das Unterpflanzen der Baumreihen mit Sträuchern eine natürliche Windschutzhecke bilden (Chalmin, 2009), wodurch der Bodenabtrag reduziert wird (Palma et al., 2005). 
Brandle et al. (2004) empfehlen auch auf feuchteren Standorten größere Abstände zur Gewährleistung einer guten Belüftung. Am häufigsten entscheidet aber die Arbeitsbreite der landwirtschaftlichen Maschinen über die Abstände zwischen den Baumreihen (Chalmin, 2009).

\subsection{Baumartenauswahl}

Um die Konkurrenz mit den landwirtschaftlichen Kulturen nach Möglichkeit so gering wie möglich zu halten und mittel- bzw. langfristig positive Effekte zu erzeugen, ist die Auswahl der Baumarten von großer Bedeutung.

Generell empfiehlt sich die Pflanzung von tiefwurzelnden Arten mit einer lichtdurchlässigen Krone (Chalmin, 2009), deren möglichst später Austrieb dem Wachstum von Winterkulturen im Frühjahr zugutekommt (Härtel, 2007).

Zur Vermeidung von erhöhtem Trockenstress auf bereits schon trockenen Standorten, sollte laut Young (1997) auch immer der Wasserverbrauch der Bäume Berücksichtigung finden.

Die verwendeten Baumarten sollten auch keine Futterpflanzen bzw. Zwischenwirte für landwirtschaftliche Schaderreger sein (Wojtkowski, 2002) wie bspw. die Robinie mit der Pflaumenschildlaus oder bei Wildobstarten der Feuerbrand (Chalmin, 2009). Auch sollten biochemische Interaktionen berücksichtigt werden, wie sie bspw. bei Schwarzund Walnüssen auftreten, die keimhemmend auf Nachbarpflanzen wirken können (Poetsch, 2001).

\subsubsection{Bewirtschaftung}

Das Hauptziel bei der Bewirtschaftung von Agroforstsystemen ist die Förderung positiver und die Vermeidung negativer Effekte. Während die Bäume anfänglich Schutz vor der Konkurrenzkraft der landwirtschaftlichen Kulturen brauchen, kehrt sich dieser Effekt im Laufe der Zeit um. (Brix et al., 2009; Chalmin, 2009)

Bei einem silvopastoralen Agroforstsystem, welches Tierhaltung auf Grünlandflächen mit der Anpflanzung von Baumreihen kombiniert und Wertholzerzeugung zum Ziel hat, ist ein zusätzlicher Baumschutz unverzichtbar. Dabei steht die Zusammensetzung der Weidetier-Arten in direktem Zusammenhang mit der Art und dem Ausmaß der ansonsten zu erwartenden Baumschäden (Machatschek, 2002).

\subsubsection{Ebenen der Bewirtschaftung}

\subsection{Unterirdisch}

Eine direkte Konkurrenz zwischen Baum und landwirtschaftlicher Nachbarpflanze um Nährstoffe und vor allem Wasser, wird durch jährlich wiederkehrende Bodenbearbeitung (Pflügen) vermieden. Die Baumwurzeln werden daher dazu angeregt, sich tiefere Bodenschichten und Ressourcen zu erschließen, die für Ackerkulturen nicht erreichbar sind. Das Herbstlaub wird durch die Bodenbearbeitung als Humus in den Oberboden eingearbeitet und wirkt somit im kommenden Frühjahr nicht mehr verdunkelnd bzw. als Brutstätte für mögliche Pilzerkrankungen (Machatschek, 2002).

Auf Grünland hingegen übt das bereits vorhandene Wurzelgeflecht, auch ohne jegliche Bodenbearbeitung, ausreichenden Konkurrenzdruck auf die noch jungen Baumwurzeln aus, auf dass diese sich in die Tiefe entwickeln (Machatschek, 2002). 


\subsection{Oberirdisch}

Mit dem Kronenwachstum der Bäume nimmt die Beschattung der landwirtschaftlichen Kulturen an den Rändern zu (siehe hierzu auch Palma et al., 2005). Das Wachstum von Pflanzenarten, die hohe Strahlungsintensitäten nutzen können, wie bspw. die C4Pflanze Mais, kann davon negativ beeinflusst werden. Bei anderen mehr schattentoleranteren Arten, die einen niedrigeren Lichtsättigungspunkt haben, wie bspw. die C3Pflanzen Raps und Weizen, zeigt sich eine Verminderung der Erträge erst bei deutlich älteren Bäumen in etwa ab dem 30. Standjahr (Härtel, 2007).

Dieser Beschattungseffekt kann aber durch Astungsmaßnahmen, die gleichzeitig die Holzqualität verbessern, im Laufe der Zeit verringert werden (Chalmin, 2009).

\subsubsection{Wirtschaftlichkeit}

Ein Agroforstsystem ist eine Kombination aus landwirtschaftlichen Kulturen mit jährlichem Ertrag und einer forstwirtschaftlichen Komponente, die erst nach Jahren bzw. Jahrzehnten meist nur einmalig einen Ernteertrag erbringt.

Es handelt sich um ein sehr dynamisches System, hervorgerufen durch das Baumwachstum, weil die Kronenentwicklung die Wachstums- und Ertragsbedingungen für die landwirtschaftlichen Kulturen ständig verändert.

Dabei ist die agroforstliche Wertholzproduktion ein eher einfaches Produktionsverfahren, was bspw. keine Spezialmaschinen erfordert. Schnellwachsende Baumarten zur Energieholzerzeugung können bereits nach drei bis fünf Jahren geerntet werden, wohingegen bei Werthölzern von einem Zieldurchmesser $>55 \mathrm{~cm}$ BHD bei einem Produktionszeitraum von 40 - 70 Jahren ausgegangen wird. (Möndel et al., 2009)

\subsection{Gegensätze in der Bewirtschaftung}

Da der Übergang zwischen Land- und Forstwirtschaft meist fließend verläuft, kommt es bei der ökonomischen Bewertung auf die Perspektive des Betrachters an.

Aus forstwirtschaftlicher Sicht werden nur genauso viele Bäume gepflanzt wie auch geerntet werden, was die Pflege- und Erziehungskosten gering hält. Weiterhin gibt es bei einer reinen Wertholzproduktion keine Vornutzungen, sondern ausschließlich die Ernte als Endnutzung.

Aus rein landwirtschaftlicher Perspektive ist der jährliche Ertrag aus den Ackerkulturen am wichtigsten. Die Bäume erzeugen Pflanz- und Pflegekosten, sorgen durch den Flächenverlust und die spätere Beschattung für Ertragseinbußen, und ein Erlös ist erst in der Zukunft zu erwarten. (Möndel et al., 2009)

Chalmin (2009) fasst diese Ambivalenz wie folgt zusammen: „Agroforstsysteme sind als Alternative erst dann interessant, wenn sie im Vergleich zu einer Monokultur höhere Flächenerträge oder andere Vorteile bieten. Ein höherer Flächenertrag ist möglich, wenn Ackerkulturen und Bäume zeitlich oder räumlich unterschiedliche Nischen besetzen und unnötige Konkurrenz vermeiden. Dadurch können vorhandene Ressourcen effizienter genutzt werden.“ 


\subsubsection{Naturschutzfachliche Betrachtungen}

Agroforstsysteme sind auf landwirtschaftlichen Flächen eine strukturelle Bereicherung, was bspw. häufig mit einer Erhöhung der Artenvielfalt einhergeht. Auf intensiv genutzten Agrarflächen werden sie seitens des Naturschutzes sogar als eine Aufwertung der Flächen betrachtet. Dabei müssen sie sich nicht zwangsläufig positiv auf die Flora und Fauna auswirken. Sie bieten aber durch die große Variabilität bei der Anlage vielfältige Möglichkeiten zur Verwirklichung verschiedenster Naturschutzmaßnahmen. (Reeg et al., 2009b)

\subsubsection{Naturschutzfachliche Bewertung}

Eine Vielzahl von Aspekten müssen für eine naturschutzfachliche Bewertung von AFS beachtet werden (Reeg et al., 2009b):

- Festlegung des Bewertungsmaßstabes: Schwerpunktsetzung bei den naturschutzfachlichen Zielen (bspw. größere Artenvielfalt, Förderung gefährdeter Arten oder Leitarten, ...) die von Bundesland zu Bundesland verschiedenen sein kann.

- Naturschutzfachliche Betrachtung der umgebenden Landschaft: handelt es sich um landwirtschaftliche Grenzstandorte oder um intensiv bewirtschafte Flächen; welche Nähe besteht zu Biotopen, usw.

- Vielfältigkeit des Systems: AFS können sehr unterschiedliche Eigenschaften und Ausprägungen haben (stark vom Bewirtschaftungsziel abhängig) die den verschiedensten Naturschutzmaßnahmen förderlich sein können.

- Dynamik bei der Entwicklung des Systems: die Lebensraumeigenschaften verändern sich durch das Wachstum der Bäume, was Veränderungen auf die Umgebung mit sich bringt.

\subsubsection{Möglichkeiten der Bewertung}

Neue Landnutzungssysteme sollten aus Sicht des Naturschutzes Probleme in der Landnutzung lösen. Auf Grünland kann jedoch die Anpflanzung von Baumreihen eine Störung bzw. Verdrängung der vorhandenen Fauna verursachen, weil Lebensräume zerschnitten werden. Auf intensiv bewirtschafteten Agrarflächen hingegen kann ein AFS durch seine Strukturelemente die Lebensbedingungen von vielen Arten verbessern. (Reeg et al., 2009b)

Der ökologische Ausgangszustand einer Fläche sollte die Grundlage zur Bewertung eines AFS bilden, denn nur wenn sich der Biotopwert steigert bzw. sich nicht verringert, sind diese für den Naturschutz von Interesse. Synergieeffekte zwischen dem Naturschutz und der Landnutzung sind jederzeit möglich. Zur biologischen Schädlingsbekämpfung bspw. benötigen Nützlinge komplexere Lebensräume als Schädlinge (Keller and Häni, 2000).

Für eine qualitative naturschutzfachliche Bewertung muss die standörtliche Gefährdungssituation von einzelnen Artengruppen Beachtung finden. (Reeg et al., 2009b) 


\subsubsection{Paulownia-Agroforstsysteme in China}

Seit den 1950er und verstärkt den 60er Jahren wird in den Huanghe- und HuaiheFlusstälern Chinas ein System aus grünen Schutzstreifen etabliert. Es soll die Schäden durch Sandstürme, Trockenheit, starke Monsunregenfälle und Winterfröste verringern und gleichzeitig den Bedarf der örtlichen Bevölkerung an Bau- und Feuerholz decken. Am häufigsten wurden dafür Baumarten wie Pappel, Ulme und natürlich Paulownia verwendet. Diese Schutzstreifen variieren in ihrer Ausdehnung (Maschenweite) zwischen 6 - 17 Hektar und umfassten schon Mitte der 1980er Jahre eine Agrarfläche in der Größe von ca. 8,7 Millionen Hektar. (Zhu et al., 1986)

\subsubsection{Allgemeine Mischungsformen}

Da die Paulownia eine Lichtbaumart ist, raten die Autoren Zhu et al. (1986) von einer Mischung mit einer anderen ebenfalls schnellwachsenden Baumart wie bspw. Populus tomentosa, Salix, Ulmus, Robinia, Ailanthus altissima (Götterbaum), Camptotheca acuminata (Chinesischer Glücksbaum) und Pterocarya stenoptera (Flügelnussbaum) ab.

Eine Kombination von Paulownia mit Nahrungspflanzen (Intercropping) erscheint hingegen sinnvoll. Dabei sollte ein Pflanzverband von $5 \times 10 \mathrm{~m}$ (200 Bäume pro ha) eingehalten werden und die erste Durchforstung nach 6-7 Jahren erfolgen. Bei einer Anlage der Reihen in Ost-West-Richtung wird der Einfluss der Beschattung der Nahrungspflanzen durch die Kronen der Bäume verringert.

Auch wurden sehr gute Ergebnisse mit einer Kombination von Paulownia und Pyllostachy pubescens (Bambus) erzielt. Durch die Schattentoleranz des Bambus und sein flacheres Wurzelsystem gedeiht dieser sehr gut, und er entwickelt sich sogar vergleichsweise schneller als Mono-Bambuskulturen.

Weitere in China oft angebaute Mischungen wären bspw. möglich mit: Fraxinus chinensis, Morus sp. (Maulbeeren), Poncirus trifoliata (Bitterorange), Trachycarpus fortunei (Chinesische Hanfpalme) und Lonicera japonica (Japanisches Geißblatt).

Auch Kräuter der chinesischen Medizin werden unter Paulownia angepflanzt, wie z.B. Paeonia spp. (Pfingstrose), (Arisaema heterophyllum Kobralilie), Codonopsis pilosula (Dang Shen, Kletterpflanze), Ophiopogon japonicus (Japanischer Schlangenbart), Chrysanthemum, Mentha piperita und Ligusticum wallichii (Szechuan-Liebstöckel). (Zhu et al., 1986)

\subsubsection{Mischkulturen}

In den 1980er Jahren wurden in China ca. 1,5 Millionen Hektar Agrarfläche in Mischung mit P.-Arten bewirtschaftet. Dies brachte nachweislich eine Verbesserung des Mikroklimas auf den Flächen, verminderte die Schäden durch Naturkatastrophen (Dürren, Stürme) und verbesserte die Versorgung und das Einkommen der Bauern durch den Erlös des Holzes bzw. die Nutzung als Brennholz.

Es lassen sich laut Zhu et al. (1986) drei Anbauvarianten anhand der PaulowniaPflanzverbände und der daraus abzuleitenden Bewirtschaftung der Feldfrüchte unterscheiden, wobei die Rotation bei der Ernte der Bäume immer 10 Jahre beträgt. 


\subsection{Primäre Holzgewinnung}

Wenn die Produktion von Holz das oberste Ziel ist und der Anbau von Feldfrüchten nur beiläufig erfolgt, dann kann ein Pflanzverband von $5 \times 5 \mathrm{~m}$ bzw. $5 \times 10 \mathrm{~m}$ gewählt werden, was zwischen 200 - 400 Bäumen pro Hektar entspricht.

Untersuchungen habe gezeigt, dass sich in den ersten drei Jahren die Feldfrüchte ganz normal entwickeln und keinen Ertragsverlust pro Flächeneinheit durch die Anpflanzung der Bäume erleiden.

Nach 4 - 6 Jahren gibt es noch immer keinen Einfluss bei Sommerfrüchten jedoch reduziert sich der Ertrag mit Herbstfrüchten signifikant.

Nach 6 - 10 Jahren können sowohl für die Sommer- als auch für die Herbstkulturen ca. $80 \%$ des sonst bei Freilandbedingungen erzeugten Ertrages erzielt werden. Das nach 10 Jahren gewachsene Holzvolumen der Paulownia-Bäume beläuft sich im Durchschnitt auf $80-140 \mathrm{~m}^{3} / \mathrm{ha}$.

\subsection{Holz und Feldfrüchte}

Wenn der Anbau von Holz und Feldfrüchten zu gleichen Teilen forciert wird, dann muss ein größerer Pflanzverband für die Bäume im Bereich zwischen $5 \times 5$ bis $15 \mathrm{~m}$ gewählt werden, was einer Anzahl von 80 - 133 Bäumen / ha entspricht.

In den ersten 5 Jahren nach der Pflanzung zeigt sich ein erhöhter Ertrag bei den Feldfrüchten im Vergleich zum Anbau unter Freilandbedingungen.

Zwischen dem 5 - 10 Jahr erhöht sich der Ertrag bei Sommerkulturen zu gleichen Teilen wie er sich bei den Herbstkulturen verringert. Über den gesamten Zeitraum von 10 Jahren betrachtet ist der Ertrag bei den Feldfrüchten in etwa gleich geblieben, und der Zuwachs an Holzmasse beläuft sich auf $36-53 \mathrm{~m}^{3} /$ ha.

\subsection{Primärer Feldfrüchteanbau}

Bei der häufigsten Anbauvariante liegt der Fokus auf den ökologischen Vorteilen, die eine Mischung mit Bäumen erbringt und der Holzzuwachs ist nebensächlich. Daher ergeben sich Pflanzverbände von $5 \times 30$ bis $50 \mathrm{~m}$ was wiederum $40-67$ Bäumen / ha entspricht. Diese Variante wird von Zhu et al. (1986) sehr ausführlich beschrieben und sie leiten aus einer großen Anzahl von Untersuchungen die folgenden Erkenntnisse ab: Die Gattung Paulownia wurzelt sehr tief und ca. $76 \%$ ihrer Wurzelmasse findet sich in einer Tiefe von $40-100 \mathrm{~cm}$ und nur ca. $12 \%$ in der für Feldfrüchte wichtigen Bodentiefe zwischen $0-40 \mathrm{~cm}$. Es entsteht folglich nur wenig Wasser- und Nährstoffkonkurrenz zwischen der Baumart und den Feldfrüchten.

Mit dem Wachstum der Bäume verringert sich der Anteil an Strahlung, die durch die Krone auf den Boden fällt. Im Alter von 7 - 8 Jahren gelangt ca. 40 - $50 \%$ der Freilandstrahlung durch die Krone. Selbst bei einer vollentwickelten und komplett belaubten Krone gelangen noch immer ca. 20 - $40 \%$ des Lichtes durch die Krone, was vergleichsweise ca. $20 \%$ höher ist als bei Populus tomentosa und $38 \%$ höher als bei Robinia pseudoacacia.

Die Paulownia treibt erst sehr spät in der Vegetationsperiode aus und behält ihre Blätter sehr lange, was anfänglich zum Vorteil für den Anbau von Sommer-Feldfrüchten ist und später selbst noch Herbst-Feldfrüchte vor Frostschäden bewahren kann. 
Aus ökonomischer Perspektive erzeugt das kombinierte Management von Bäumen und Feldfrüchten, bspw. durch die Düngung, bessere Wachstumsbedingungen für die Paulownia, was sich anhand eines erhöhten Durchmesser- und Holzuwachses im Vergleich zu Plantagenbäumen messen lässt. Zusätzlich dazu produziert eine Paulownia im Alter zwischen 8 - 10 Jahren jährlich ca. $100 \mathrm{~kg}$ frisches Blattmaterial was in etwa $28 \mathrm{~kg}$ Trockenmaterial entspricht und als Viehfutter oder Dünger für die Felder verwendet werden kann. Bei einem Umtrieb von 10 Jahren bietet ein Baum im Durchschnitt nicht nur ca. 0,4-0,5 $\mathrm{m}^{3}$ Stammholz, sondern zusätzlich noch $350-400$ $\mathrm{kg}$ Astmaterial, was der örtlichen Bevölkerung als Brennholz dienen kann. (Zhu et al., 1986) 


\section{Kapitel III}

\section{Freiland- und Gewächshausversuche}

\subsection{Methodik}

\subsubsection{Allgemeine Methodik}

\subsubsection{Literaturrecherche}

Die hier verwendete Literatur, speziell in den Kapiteln I und II soll eine Übersicht bieten und mit Hilfe der Experimente des Kapitel III das Wissen innerhalb des Themengebietes erweitern (Boote and Beile, 2005; Cook et al., 1997).

Ein Großteil der historischen als auch aktuellen nicht-europäischen Literatur zu dieser Thematik steht nur unzureichend in Form von englischsprachigen Abstracts bzw. Summarys zur Verfügung. Es ist schwer abschätzbar wie viel Literatur bzw. Quellen ausschließlich in chinesischer (Mandarin, Hochchinesisch) bzw. japanischer Sprache verfasst sind und daher aus westlicher Hemisphäre nur begrenzt bzw. gar nicht zugänglich sind.

Die Anzahl westlicher Literaturquellen hat sich aber in den letzten 40 Jahren deutlich erhöht, was den erhöhten Forschungsdrang zu dieser Baumart in den verschiedenen Ländern widerspiegelt (1.1.1.4.1 Wiedereinführung auf dem amerikanischen Kontinent).

Die verwendeten Quellen entstammen verschiedenen Sammlungen und elektronischen Datenbanken und Bibliotheken, wie z.B. Forestry Compendium von CABI.org.

Als Suchmaschinen wurden ausschließlich die folgenden verwendet: Ecosia, DuckduckGo, Google und vor allem GoogleScholar. Auch wurde auf Homepages von diversen Firmen wie bspw. WeGrow (2020) oder Cathaia (2020) zugegriffen und daraus hinlänglich zitiert.

\subsubsection{Datengrundlage}

Eine Datenerhebung bildet im allgemeinen die Grundlage für eine spätere statistische Auswertung (Bourier, 2013). In Abhängigkeit von dem jeweiligen Experiment bzw. Versuchsaufbau erfolgte die Erhebung zu unterschiedlichen Zeitpunkten und mittels verschiedener Messinstrumente. Die Messungen wurden mit einem Zeitstempel versehen und alle Daten wurden in Exceltabellen hinterlegt und als sogenannte „Urtabellen“ gespeichert und diesem Manuskript digital beigefügt.

Zur Auswertung von Klimadaten wurde auf die allgemeinzugänglichen Daten des Klimaarchivs zurückgegriffen. Dieses Archiv wird vom zivil meteorologischen Dienst der Bundesrepublik Deutschland, dem Deutschen Wetterdienst (DWD), bereitgestellt. Es verfügt über ein nationales, relativ engmaschiges Netz von meteorologischen Messinseln und durch die allzeit zugänglichen Daten, können die in dieser Arbeit vorgenommen Auswertungen jederzeit überprüft werden. 


\subsubsection{Statistische Auswertung}

Neben einer gezielten Datenauswertung, zur Beantwortung der jeweiligen Fragestellung, wurde auch immer wieder ein explorativer Ansatz gewählt, mit der Hoffnung den Daten vielleicht noch ein wenig mehr an Informationen und Zusammenhängen zu entlocken. Dadurch zeigten sich aber auch schnell die Grenzen und Möglichkeiten der erhobenen Daten, die leider nicht immer für jede gewünschte Auswertung ausreichend waren.

Wenn auf Ergebnisse der BSc- und MSc-Abschlussarbeiten Bezug genommen wird, so wird dies unter Nennung der Quelle extra erwähnt. Ansonsten sind alle dargestellten Ergebnisse auf Grundlage der vorhandenen Daten selbstberechnet und in ihrer Darstellung und Schlussfolgerung völlig unabhängig von der jeweiligen Abschlussarbeit zu betrachten.

Zur Erhebung, Verzeichnung und Speicherung aller Daten wurde das Tabellenkalkulationsprogramm MS Excel von Microsoft, Version 14.0 (Excel2011) verwendet.

Die Datenpakete des Deutschen Wetterdienstes wurden aufgrund der leichteren Verfügbarkeit und Kompatibilität mittels dieser Software bearbeitet, und es wurden sowohl Grafiken als auch Tabellen damit erstellt.

Zur weiteren Datenbearbeitung und Auswertung wurde eine Privatlizenz der Statistiksoftware IBM SPSS Statistics, Version 19, verwendet.

Alle Datensätze wurden vorab auf Normalverteilung geprüft. Dies erfolgte sowohl optisch durch Histogramme und Normalverteilungskurven und mittels Q-Q-Diagrammen bzw. trendbereinigten Q-Q-Diagrammen, als auch rechnerisch durch Überprüfung der Schiefe und Kurtosis (Werte nahe Null), und mit Hilfe von Kolomogorov-Smirnov-Tests und Shapiro-Wilk-Tests (Überprüfung der Signifikanz). Bei allen angewandten statistischen Tests wurde vorab eine maximale Irrtumswahrscheinlichkeit (Signifikanzniveau) von 0,05 festgelegt.

Bezüglich der Gewächshausversuche wurden in Abhängigkeit von den darzustellenden Ergebnissen die Daten umsortiert und ggf. Pflanzen, die im Wachstumsprozess abgebrochen bzw. ausgefallen waren, nur bis zum besagten Ereignis statistisch berücksichtigt. Dadurch ändert sich im Laufe des Beobachtungszeitraumes und bei den jeweiligen Ergebnisdarstellungen der Stichprobenumfang.

\subsubsection{Freilandversuche}

\subsubsection{1 Übersicht}

Im Mai 2015 wurden drei Versuchsflächen in den Bundesländern Brandenburg, Niedersachsen und Schleswig-Holstein angelegt (Abb. 11). Die Flächen wurden dabei so ausgewählt, dass ein möglichst großes Spektrum an verschiedenen Böden und klimatischen Bedingungen abgebildet werden konnte.

Der Standort der Brandenburger Versuchsfläche (im Folgenden nur noch Brandenburg (BB) genannt) wird von pleistozänen Sanden geprägt (Tab. 6), und es herrscht ein trockenes Kontinentalklima.

Die Versuchsfläche auf der Nordseeinsel Föhr wird von Marschböden und einem feuchten, atlantischen Klima mit vergleichsweise hohen Niederschlägen geformt. 
Auf der Versuchsfläche in Göttingen (im Folgenden nur noch Göttingen (Gö) genannt) herrscht ein eher atlantisch / kontinentales Übergangsklima und mit flachgründigem Kalk als geologisches Ausgangsgestein.

Auf allen drei Versuchsflächen wurden zeitgleich die Arten: P.elongata, P.fortunei, P.tomentosa, in gleicher Stückzahl gepflanzt. Da die Fläche in Brandenburg doppelt so groß ist wie die auf Föhr und in Göttingen, konnte hier die Anzahl an Pflanzen und somit auch der Stichprobenumfang verdoppelt werden. Alle im Freiland angebauten P.Pflanzen entstammen einer auf die Gattung Paulownia spezialisierten Baumschule (Cathaia, 2020). Die Pflanzen wurden dort im Frühjahr 2015 aus Samen angezogen und für das Experiment angekauft und nach Göttingen geliefert, von wo aus sie auf die Versuchsflächen ausgebracht

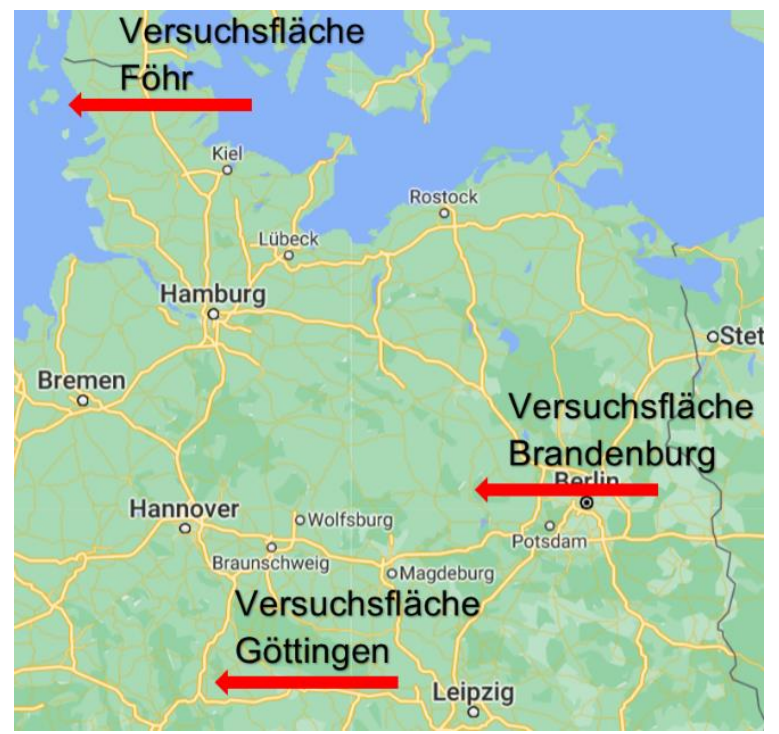

Abb. 11: Standorte der Versuchsflächen, Quelle: eigene Darstellung basierend auf Google Maps. wurden.

\subsection{Ziele des Experiments}

In diesen mehrjährigen Versuchen sollten die Wachstumsreaktionen der drei P.-Arten unter mitteleuropäischen Klimabedingungen beobachtet werden. Dabei sollten die Annahmen überprüft werden, ob die klimatischen und standörtlichen Bedingungen das Anwuchsverhalten und das Längenwachstum der Pflanzen beeinflussen. Darüber hinaus wurden die Pflanzen in vier verschiedene Behandlungsarten unterteilt, um zu überprüfen ob Bodenhilfsstoffe wie Geohumus oder ein Mykorrhizierung Einfluss auf das Längenwachstum und die Höhe der Ausfallrate nehmen. Des Weiteren war davon auszugehen, dass sich Unterschiede beim Wachstumsverlauf im Vergleich zu dem natürlichen Verbreitungsgebiet der Arten zeigen würden. Auch würden sich arttypische Unterschiede beim Wachstum herausbilden und sich von Vegetationsperiode zu Vegetationsperiode weiter verstärken.

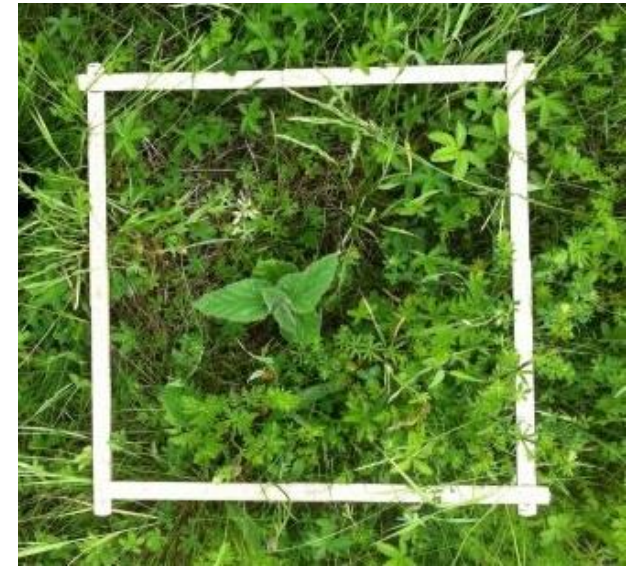

Abb. 12: Paulownia-Pflanze umringt von Begleitwuchs, auf der Versuchsfläche in Göttingen.

\subsubsection{Aufbau der Versuchsflächen}

Alle Versuchsflächen wurden, hintereinander binnen weniger Tage im Mai 2015, in der Reihenfolge - zuerst Göttingen, dann Brandenburg und schließlich Föhr errichtet und bepflanzt (Abb. 13). 
Die Flächen in Göttingen und Föhr haben jeweils die Maße $20 \times 50 \mathrm{~m}$ und sind somit 1.000 qm groß. Die Brandenburger Versuchsfläche weist mit 2.000 qm neben der doppelten Größe auch die doppelte Plot- und Pflanzenanzahl auf.

Alle Flächen wurden mit 1,8 m hohem handelsüblichen Forstzaun (Knotengeflecht 180/16/15) zum Schutz vor Wild und menschlicher Einflussnahme umzäunt.

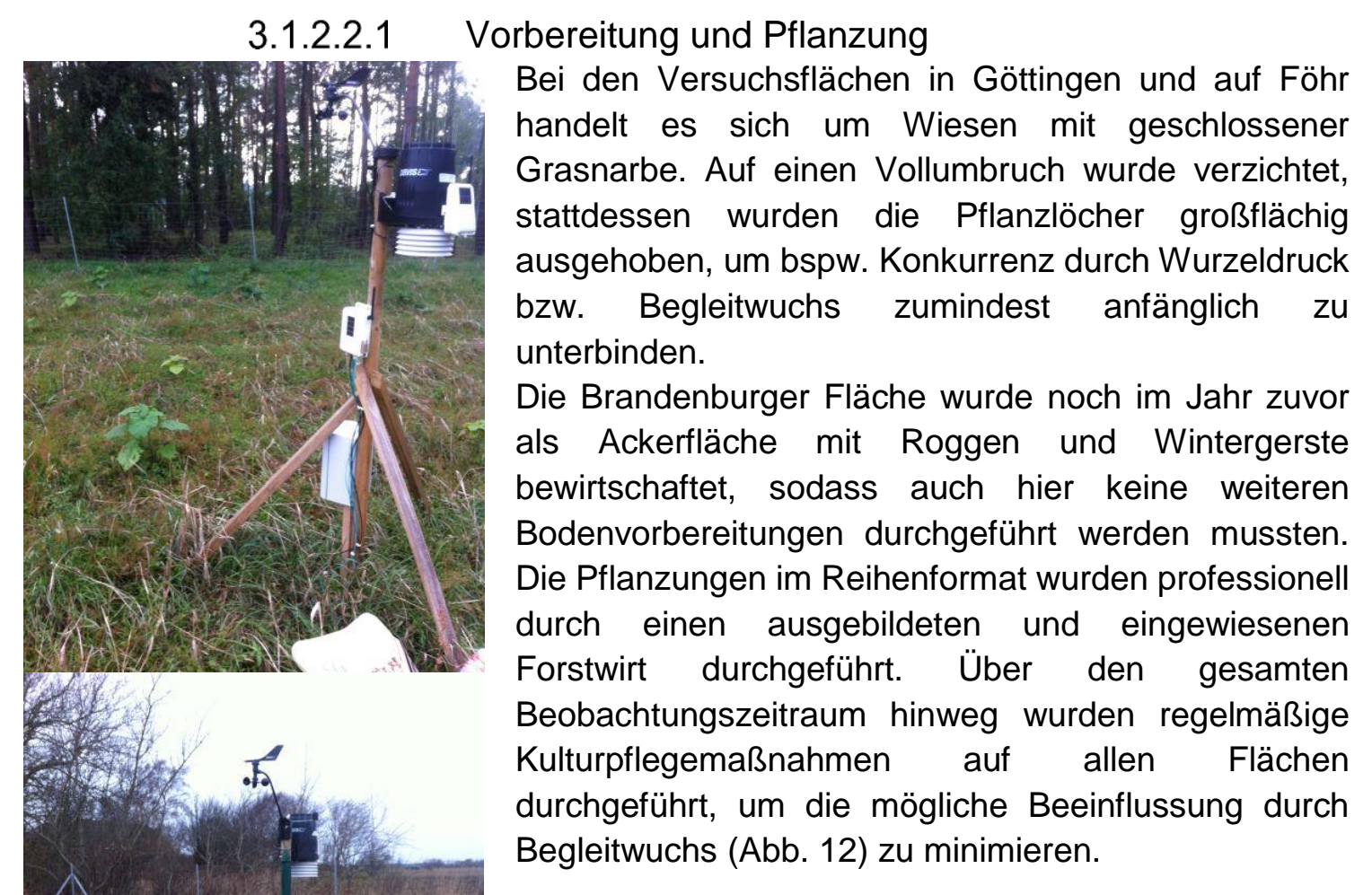

3.1.2.2.2 Versuchsflächen-Design

Insgesamt wurden pro Versuchsfläche (in Brandenburg mit doppelter Anzahl) 24 Paulownia-Plots in Reihenpflanzung angelegt. Diese wurden vorab noch einmal in die folgenden vier Behandlungsgruppen unterteilt:

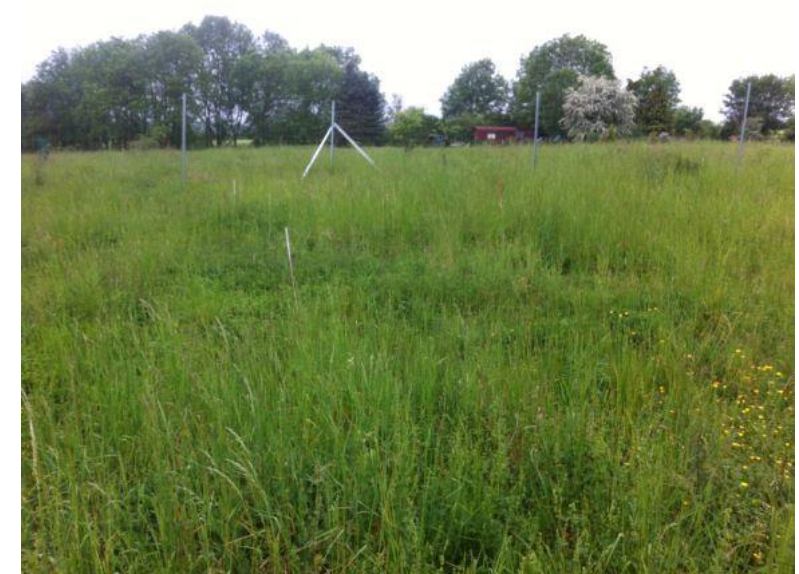

$\mathrm{N}$ = unbehandelt (Nullfläche) ohne Zusätze von Bodenhilfsstoffen,

$\mathrm{G}=$ Geohumus, Zugabe von $100 \mathrm{ml}$ Geohumusgranulat in das Pflanzloch

M = Mykorrhiza, Zugabe von $100 \mathrm{ml}$ Mykorrhizapilz-Konzentrat

$\mathrm{GM}=$ Zugabe von $100 \mathrm{ml}$ Geohumusgranulat und $100 \mathrm{ml}$ MykorrhizapilzKonzentrat.

Abb. 13: V.o.n.u. Funkwetterstationen auf der Brandenburger-, Föhrer- und der Göttinger Versuchsfläche. 
Zur Vermeidung systematischer Fehler wurden die Plots nach dem Zufallsprinzip über die Fläche verteilt (Abb. 51). Jeder Plot wird von vier Pflanzen der gleichen Baumart gebildet, die im Verband von $2 \times 2 \mathrm{~m}$ gepflanzt und völlig gleich behandelt wurden. Die Pflanzen eines Plots haben zu den Nachbarpflanzen somit immer einen Mindestabstand von $2 \mathrm{~m}$.

Auf Föhr und in Göttingen konnten diese Plots mit zweifacher und in Brandenburg mit vierfacher Wiederholung gepflanzt werden.

\subsection{Bodenhilfsstoffe}

Das körnige Substrat Geohumus hat eine Größe von 0,5 - $7 \mathrm{~mm}$ und besitzt sowohl quellende als auch schrumpfende Eigenschaften. Bei Kontakt mit Wasser reagiert das Granulat wie ein Schwamm und gibt das Wasser beim Schrumpfungsprozess gleichmäßig wieder ab. Die Bezeichnung Geohumus ist ein nicht geschützter Begriff der vom Hersteller als Produktbezeichnung gewählt wurde ("Datenblatt geohumus.pdf," n.d.) (Abb. 49).

Laut Herstellerangaben steigert der Bodenhilfsstoff die Wasserhaltekapazität von Böden und Substraten, wobei das Quellen und Schrumpfen des Granulats die Luftversorgung der Wurzeln verbessern soll.

Die Dosierung liegt bei $10 \mathrm{ml}$ Granulat pro Liter Bodenvolumen, was auf die Größe der Pflanzlöcher angepasst wurde. Zusammengesetzt ist das Substrat aus Lavagesteinsmehl, Tonmineralien, Bentonit und Sand sowie: $5,8 \quad \% \quad$ Magnesiumoxid (MgO)

$2,8 \quad \% \quad$ Gesamtcalciumoxid $(\mathrm{CaO})$

$1 \%$ Gesamtstickstoff $(\mathrm{N})$

$0,72 \%$ Gesamtkaliumoxid $\left(\mathrm{K}_{2} \mathrm{O}\right)$

$0,42 \%$ Gesamtphosphat $\left(\mathrm{P}_{2} \mathrm{O}_{5}\right)$

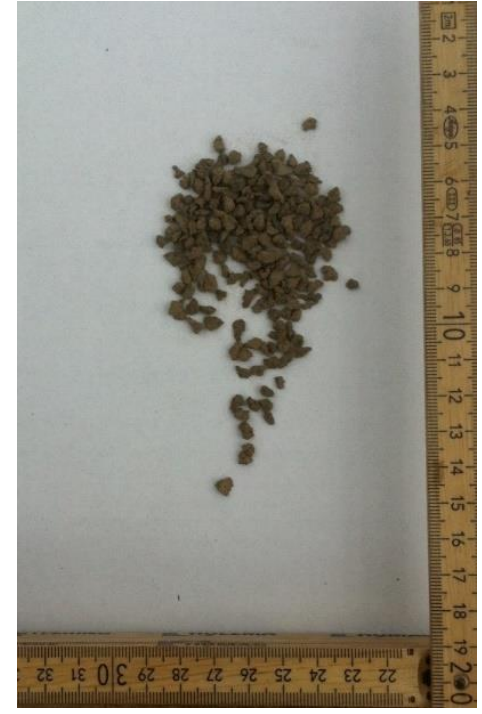

Abb. 14: Geohumus Granulat, verändert nach Büker (2015).

Es enthält ein wasserunlösliches Polyacrylat (ein aus Estern der Acrylsäure hergestelltes Polymere), welches als Superabsorber dient (Abb. 14).

Die Wasseraufnahmefähigkeit entspricht $40 \mathrm{ml}$ pro Gramm Geohumus bei freier Quellung, wobei das Wasser zu über $95 \%$ pflanzenverfügbar sein soll. Bei einem Pflanzloch von 10 Liter Bodenvolumen, welches mit $100 \mathrm{ml}$ Granulat angereichert wurde, sollte dies einer zusätzlichen Wasserspeicherkapazität von bis zu 4 Litern Wasser entsprechen.

Der Hersteller verspricht auf seiner Internetseite, dass die Pflanzen durch die Verwendung des Granulats um bis zu $30 \%$ weniger gegossen werden müssen als normalerweise (Geohumus, 2020).

Wie Untersuchungen von Mehrotra et al. (1998) gezeigt haben, erhöht sich das Höhen-, das Durchmesser- und somit auch das Biomassewachstum von künstlich mit Endomykorrhiza infizierten P.-Pflanzen. Auch die Überlebensrate von Sämlingen soll sich durch diese Symbiose steigern (Mehrotra, 1996).

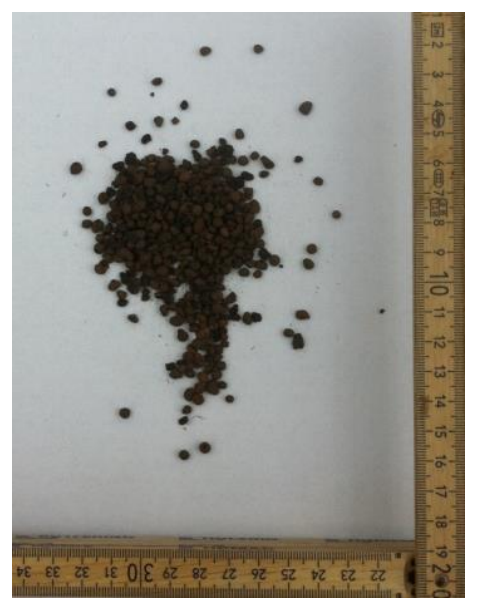

Abb. 15: MykorrhizapilzKonzentrat, verändert nach Büker (2015). 
Um diese Ergebnisse überprüfen zu können, wurde eine Behandlungsgruppe mit einem Mykorrhizapilz-Konzentrat der Firma BioMyc ${ }^{\mathrm{TM}}$-Environment $\mathrm{GmbH}$ präpariert (Abb. 15). Es besteht aus ca. $2-4 \mathrm{~mm}$ großen Blähtonkügelchen die mit den Pilzsporen der Gattung Glomus (Ordnung: Jochtrüffelartige = Glomerales, Klasse: Arbuskuläre Mykorrhizapilze) geimpft wurden. Laut Herstellerangaben wird eine Dosierung von $10 \mathrm{ml}$ Konzentrat pro Liter Bodensubstrat empfohlen, wonach sich gerichtet wurde.

\subsection{Erfassung der Klimabedingungen}

Zur Erfassung des standörtlichen Klimas wurde jede Versuchsfläche mit einer FunkWetterstation der Marke „Vantage Pro2“ ausgestattet. Diese verfügt über Bodenfeuchtesensoren, Mehrzweck-Temperaturfühler und Blatt- / Bodenfeuchte Transmitter.

Diese Wetterstationen zeigten jedoch eine hohe Fehleranfälligkeit und den häufigen Verlust von Daten, welcher nur unzureichend kompensiert werden konnte.

Somit wurden zur weiteren Auswertung der Klimabedingungen ausschließlich die freiverfügbaren Klimadaten des Deutschen Wetterdienstes herangezogen. Dies hat zum Vorteil, dass die Vergleichbarkeit der Klimabedingungen vereinfacht wird, da vereinheitlichte Daten vorliegen.

Die für die Auswertung in Frage kommenden Wetterstationen des DWD sind Genthin in Sachsen-Anhalt (Stationsnummer: 1605), Göttingen in Niedersachsen (SN: 1691) und List auf Sylt in Schleswig Holstein (SN: 3032), da sie alle geographisch am nächsten an der jeweiligen Versuchsfläche gelegen sind.

\subsubsection{Gewächshausversuche}

\subsubsection{1 Übersicht}

Aufbauend auf den ersten Erfahrungen der Freilandversuche und zur Ergänzung der Fragestellungen wurde im Frühjahr 2016 ein Gewächshausversuch an der GeorgAugust-Universität Göttingen, Fakultät für Forstwissenschaften und Waldökologie, Abteilung Waldbau und Waldökologie der Gemäßigten Zonen konzipiert.

Es sollte das Wachstumsverhalten der drei P.-Arten: P.elongata, P.fortunei und P.tomentosa bei unterschiedlichen Beschattungs- und Bewässerungsstufen beobachtet werden. Hierfür wurden insgesamt 540 P.-Pflanzen, in 3 Arten, 3 Wasser- und 3 Lichtstufen aufgeteilt und für die Dauer von zwei Vegetationsperioden beprobt.

\subsection{Ziele des Experiments}

Es sollten die Annahmen überprüft werden, ob bei einer Verringerung des Lichtes das Längenwachstum zunimmt, das Durchmesserwachstum abnimmt und gleichzeitig weniger Biomasse gebildet wird. Des Weiteren war davon auszugehen, dass eine Verringerung der Wasserzufuhr ein Rückgang des Längenwachstums des Durchmessers und der Biomasse zur Folge hätte.

Die P.-Arten würden aller Voraussicht nach unterschiedlich auf die verschiedenen Behandlungsarten- und -stufen reagieren und diese Unterschiede würden sich von der ersten zur zweiten Vegetationsperiode verstärken. 


\subsubsection{Aufbau der Gewächshausversuche}

Der Zeitraum der Versuche erstreckte sich vom April 2016 bis zum Oktober 2017. Zur Durchführung wurde ein überdachtes, vollverglastes, aber nach außen offenes Gewächshausabteil der Universität Göttingen genutzt. Auf diese Weise konnten die natürlichen Witterungsbedingungen wie Temperatur, Luftfeuchtigkeit und die gesamte Sonneneinstrahlung ungehindert die Pflanzen erreichen. Jeglicher Einfluss durch Niederschläge wurde ausgeschlossen und stattdessen eine kontrollierte Wasserversorgung durchgeführt.

Für alle im Experiment verwendeten P.-Pflanzen kann Artreinheit garantiert werden, da sie aus einer auf die Gattung Paulownia spezialisierten

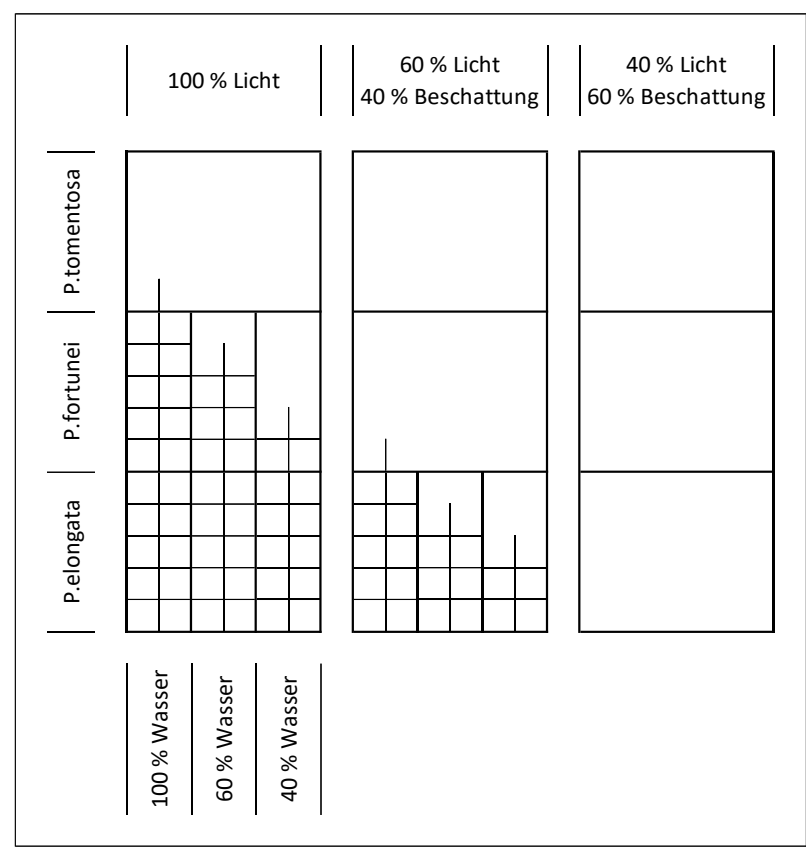

Abb. 16: Schematischer Aufbau der Pflanztische Unterteilung in 3 P.-Arten (P.elongata, P.fortunei, P.tomentosa), 3 Lichtstufen (100\%, $60 \%, 40 \%)$ und 3 Wasserstufen (100\%, $60 \%, 40 \%)$.

Baumschule stammen (Cathaia, 2020). Die Pflanzen wurden dort im Frühjahr 2016 aus Samen angezogen und für das Experiment angekauft.

Nach Abschluss der ersten Vegetationsperiode wurde die oberirdische Stammbiomasse geerntet und die Pflanzen somit auf den Stock gesetzt. Zum einen ist dieses Verfahren, beim Anbau der Pflanzen in Form von Kurzumtriebsplantagen, üblich (Zhu et al., 1991) und zum anderen ermöglicht dies den zusätzlichen Gewinn an wissenschaftlichen Daten bezüglich der ersten Vegetationsperiode.

Im Freiland sollen sich die Pflanzen im ersten Jahr auf dem Standort etablieren. Das auf den Stock setzen vor Beginn der zweiten Vegetationsperiode ermöglicht den Austrieb eines neuen Terminaltriebes der sich laut Stimm et al. (2013) stärker und geradliniger entwickelt.

\subsection{Pflanzung}

Die Pflanzen wurden als Containerware angeliefert und, mit dem von der Baumschulerde umhüllten Wurzelballen, in ein einheitliches Substrat (Einheitserde, EUROHUM) verpflanzt. Alle Töpfe haben die Maße 13,7 x 13,7 x $23 \mathrm{~cm}$ und fassen ein Bodenvolumen von 3 Litern.

Um den Nährstoffverlust des Bodensubstrats aus der ersten Vegetationsperiode auszugleichen, wurden zu Beginn des zweiten Jahres alle Pflanzen mit der gleichen Menge des GEPAC LZD-Langzeitdüngers der Firma Gebr. Patzer GmbH \& Co KG gedüngt. 


\subsection{Behandlungsarten}

Für den Grundaufbau des Versuches wurden 270 Pflanzen auf 3 Tischen in Reihen zu 6 x 15 Töpfen gleichmäßig angeordnet. Dabei stehen auf jedem Tisch immer 30 Pflanzen der gleichen P.-Art (P.elongata, P.fortunei, P.tomentosa) was sich folglich auf 90 Pflanzen pro Tisch aufsummiert.

Des Weiteren wurden alle Pflanzen in drei verschiedene Bewässerungs- und Beschattungsarten unterteilt, wobei die Abstufungen immer von $100 \%$ über $60 \%$ zu 40 $\%$ erfolgten. Jeder Tisch entspricht einer Belichtungsstufe, aber es kommen pro Tisch immer alle drei Bewässerungsstufen zur Anwendung. Folglich wurden pro Tisch jeweils 10 Pflanzen einer Art der gleichen Bewässerungsstufe unterzogen (Abb. 16).

Um den Stichprobenumfang zu erhöhen und schematische Fehler zu vermeiden, wurde die Anzahl der Tische auf 6 verdoppelt und so auch die Pflanzenanzahl auf 540 erhöht. Somit wurden insgesamt immer 20 Pflanzen ein und derselben Behandlungsvariante unterzogen, wobei es insgesamt 27 verschiedene Behandlungsvarianten (3 Pflanzenarten $\times 3$ Lichtstufen $\times 3$ Wasserstufen) gibt.

\subsection{Bewässerung}

In beiden Vegetationsperioden wurde das gleiche Bewässerungsschema verwendet. In der Vegetationsruhe wurde durch regelmäßige Kontrollen garantiert, dass das Bodensubstrat nicht austrocknete und gleichzeitig nicht zu feucht wurde, um ein Absterben der Pflanzenstöcke zu verhindern.

Die Einteilung der Bewässerungs-stufen orientiert sich an den realen Niederschlagsbedingungen in zwei unterschiedlichen Wuchsregionen Deutschlands. Die 100 \% Bewässerungsstufe entspricht dabei der Region Rheinland-Pfalz, die nach Mosandl \& Stimm (2015) durch ihr Weinbauklima besonders geeignet für den P.-Anbau ist, da sie sowohl über einen hohen Jahresniederschlag innerhalb der Vegetationszeit, als auch ein warmes Klima verfügt.

Im Vergleich dazu entspricht die Niederschlagsmenge des Landes Brandenburgs in etwa $60 \%$ des Rheinland-Pfälzischen Wertes und wird daher als zweite Bewässerungsstufe verwendet.

Um das Extrema für die P.-Pflanzen weiter zu erhöhen und sie bspw. auf mögliche Folgen des voranschreitenden Klimawandels zu untersuchen, wurde die letzte Bewässerungsstufe auf $40 \%$ des Jahresniederschlages von Rheinland-Pfalz reduziert.

Der Jahresniederschlag ${ }^{17}$ wurde auf Basis der Daten des Deutschen Wetterdienstes (DWD, 2020) berechnet. Hierfür wurden die Freilandjahresniederschläge der Jahre 1981 bis 2010 gemittelt und der Wert von Millimeter pro Quadratmeter schließlich auf die eigentliche Flächengröße im Pflanztopf und auf Milliliter als Volumeneinheit umgerechnet.
Tab. 4: Wassermengen der drei Bewässerungsstufen.

\begin{tabular}{|l|r|r|r|}
\hline & \multicolumn{3}{|c|}{ Wassermenge in mm } \\
\hline Monat & $100 \%$ & $60 \%$ & $40 \%$ \\
\hline April / Mai & 135 & 80 & 50 \\
\hline Juni / Juli & 150 & 90 & 60 \\
\hline August / September & 145 & 85 & 55 \\
\hline Gesamtmenge pro a: & 833 & 500 & 333 \\
\hline
\end{tabular}

\footnotetext{
17 Die Niederschläge werden in Millimeter pro Quadratmeter angegeben. Dabei entsprechen bspw. 833 mm/a einer Niederschlagsmenge von 833 Litern auf einer Fläche von einem Quadratmeter für den Zeitraum eines ganzen Jahres.
} 
Die Variation der Niederschläge im Laufe der Vegetationsperiode wurde berücksichtigt und die Gießmenge dementsprechend angepasst (Tab. 4). Zur Fehlervermeidung und Vereinfachung bei der Durchführung des Gießvorganges wurde auf $5 \mathrm{ml}$ Werte gerundet. Die Anzahl der Regenereignisse pro Monat variiert ebenfalls stark und wurde schließlich auf zwei Gießvorgänge pro Woche festgelegt, was auch am ehesten den Freilandbedingungen entspricht.

\subsection{Beschattung}

Die Einteilung der Beschattung wurde zur leichteren Vergleichbarkeit, genau wie bei der Bewässerung, in drei Stufen eingerichtet. Dabei wurde die Abgrenzung zwischen den Lichtstufen mittels Beschattungsnetzen realisiert, wie sie in Baumschulen üblicherweise Verwendung finden. Die Netze wurden dazu über ein dünnes Holzgestell gespannt, in der Mitte an der Decke befestigt und umhüllten die Tische somit zu allen Seiten.

Der erste Tisch wurde mit keinem Netz ausgestattet und hat den Lichtwert $100 \%$, welcher der gesamten, durch das Gläserdach des Gewächshauses einfallenden, Sonneinstrahlung entspricht. Am zweiten Tisch ist der $60 \%$ Lichtwert gleichbedeutend mit einer $40 \%$ Beschattung und wurde durch ein relativ grobmaschiges Netz ermöglicht (Abb. 17).

Der dritte Tisch wurde durch ein sehr feinmaschiges Netz auf einen Lichtwert von $40 \%$ reduziert, was einer $60 \%$ Beschattung entspricht.

Durch die Verdopplung der Tisch- und Pflanzenanzahl gab es folglich im Gewächshaus immer zwei Tische mit der jeweils gleichen Licht- bzw.

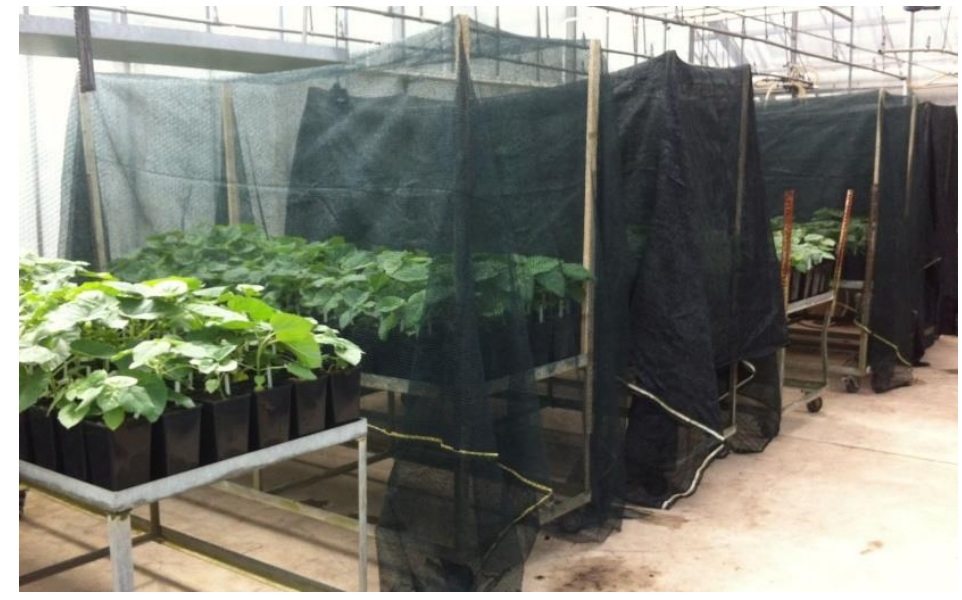

Abb. 17: Aufbau der Pflanztische im Gewächshaus; links im Vordergrund der Tisch mit $100 \%$ Licht, gefolgt vom $60 \%$ Lichtund $40 \%$ Licht-Tisch und anschließend noch einmal wiederholt. Beschattungsstufe.

\subsubsection{Datenerhebung}

Das Längenwachstum der Pflanzen wurde mit einem Gliedermaßstab bzw. einem Maßband auf einen halben Zentimeter genau, vom Boden bis zur Spitze des Terminaltriebes vermessen.

Die Blattanzahl der Pflanzen wurde durch einfaches Abzählen der noch lebenden, nicht verwelkten Blätter an der Pflanze ermittelt.

Der Wurzelhalsdurchmesser (WHD) wurde mit einem elektronischen Messschieber, ca. $5 \mathrm{~cm}$ über dem Bodensubstrat auf $1 / 10 \mathrm{~mm}$ genau ermittelt. Dies erfolgte in Form einer Kreuzkluppung, da die Stängel der P.-Pflanzen eine leicht ovale Wuchsform aufweisen. Die beiden Messungen erfolgten im rechten Winkel zueinander und wurden anschließend gemittelt. Generell wurde zum Abschluss der Vegetationsperiode der Pflanzendurchmesser in den Höhen von 5, 10, $20 \mathrm{~cm}$ und dann in Abständen von $20 \mathrm{~cm}$ weiter bis zum Ende des Terminaltriebes erfasst. 
Nach dem Ende der Vegetationszeit wurde die gesamte oberirdische Biomasse mit Ausnahme der abgefallenen Blätter, in etwa einen Zentimeter oberhalb des Wurzelhalsdurchmesser (WHD), abgeerntet bzw. auf den Stock gesetzt (Röhle, 2013) (Abb. 18).

Das Pflanzenmaterial wurde in labortypische Papiertüten verpackt und für zwei Tage bei $105{ }^{\circ} \mathrm{C}$ in einem Trocknungsschrank behandelt.

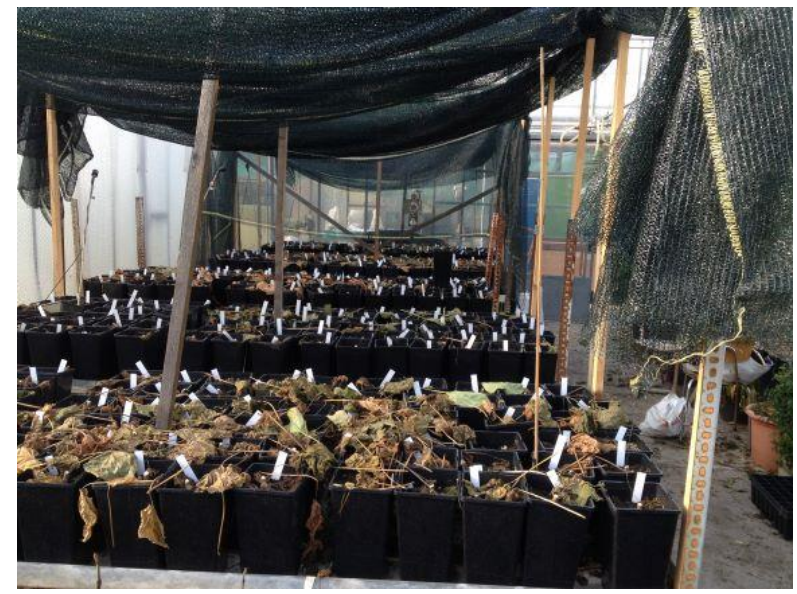
Anschließend erfolgte die Gewichts- Abb. 18: Pflanztische im Gewächshaus - abgeerntet. ermittlung, zur Vermeidung eventueller Luftbewegungen in einem Waagen-Kasten, mit einer Messgenauigkeit auf $10^{-3} \mathrm{~g}$ genau.

Zum Ende der zweiten Vegetationsperiode wurde eine Vollaufnahme aller lebenden Blätter durchgeführt. Diese erfolgte zeitlich noch vor dem natürlichen Laubabfall der Pflanzen. Dabei wurden die Länge, die Breite und der Stiel eines jeden Blattes vermessen.

Die Länge wurde von der Blattspitze bis zum Stielansatz mittels eines Maßbandes ausgemessen. In die Mitte des Blattes wurde eine gedachte horizontale Linie gelegt und auf ihr die Breite des Blattes erfasst.

Anschließend wurde ein Stichprobenumfang von 60 Blättern abgeerntet und eingescannt. Der Flächeninhalt der gescannten Blätter wurde mit dem Bildbearbeitungsund Bildverarbeitungsprogramm „ImageJ“ ermittelt. Basierend auf diesen Daten wurden mehrere Regressionsanalysen durchgeführt, bei denen sich zeigte, dass der Blattdurchmesser in direktem Zusammenhang zum Blattflächeninhalt steht.

\subsection{Sensoren}

Um die klimatischen Bedingungen der Pflanzen im Gewächshaus zu überprüfen, wurden die Tische mit Messsensoren ausgestattet.

Hierfür kamen drei HOBO-Micro-Station-Datenlogger zum Einsatz. Sie sollten die Daten der

- Licht-(Photosynthese)-Sensoren (PAR-Strahlung),

- der Bodenfeuchte-Sensoren (Bodenwassergehalt),

- der Bodentemperatur-Sensoren und

- der Temperatur / Luftfeuchte-Sensoren aufzeichnen.

Die Haltbarkeit der Datenlogger erwies sich als mangelhaft und durch ihren mehrfachen Ausfall konnten nur unzureichend Daten erfasst werden.

Die Gewächshauspflanzen waren durchgängig den normalen klimatischen Bedingungen des Offenlandes ausgesetzt und nur der Einfluss von natürlichem Niederschlag wurde vermieden. Auch die genaue Wassermenge zur Bewässerung wurde jederzeit erfasst. Daher wird im weiteren Verlauf auf die Daten der Logger verzichtet und ausschließlich auf freizugängliche Wetterdaten des Deutschen Wetterdienstes (DWD, 2020) Bezug genommen. 


\subsection{Ergebnisse}

Die Anwuchsphase von Pflanzen ist bei jeder Kulturbegründung besonders kritisch. Die Pflanzen müssen den sogenannten Pflanzschock überwinden und mit ihren Wurzeln den Boden erschließen und dabei möglichst schnell in die tieferen, feuchteren Bodenschichten vordringen. In den letzten Jahren hat sich diese kritische Phase, durch das immer häufigere Auftreten von saisonalen Trockenereignissen, weiter verschärft.

Die beiden Versuchsgruppen im Freiland und im Gewächshaus beantworten unterschiedliche und sich ergänzende Fragestellungen zu dieser Problematik. Vor allem die natürlichen ökologischen Rahmenbedingungen, die diese Anwuchsphase bestimmen, lassen sich sehr gut im Freiland untersuchen. Insofern wird dieser Abschnitt vorangestellt.

\subsubsection{Zum Anbau im Freiland}

Für den Anwuchserfolg ist eine Vielzahl von Faktoren entscheidend, wie bspw. die Baumart, deren Provenienz, das Pflanzmaterial und der Zustand der Pflanzen bzw. deren Transport und Lagerung, um nur einige zu nennen. Besonders wichtig sind aber vor allem die Witterungsbedingungen zum Zeitpunkt der Kulturbegründung und in den Wochen bzw. Monaten danach.

Verglichen werden hier im Folgenden die drei unterschiedlichen Arten der Gattung Paulownia (P.elongata, P.fortunei und P.tomentosa). Das Pflanzmaterial selbst entstammt aus einer auf diese Baumart spezialisierten Baumschule (Cathaia, 2020) und war dadurch sehr homogen und wies folglich keine größeren Variationen auf. Auch die eigentliche Pflanzung, mittels Lochspaten, wurde für alle Varianten identisch, von einem geschulten Forstwirt durchgeführt. Insofern kam der Variable der ökologischen Rahmenbedingungen, bestehend aus Witterung, Bodenverhältnissen und hier insbesondere der nutzbaren Wasserkapazität, die größte Bedeutung für den Pflanzerfolg $\mathrm{zu}$.

\subsubsection{Ausfallrate im Freiland}

Das Jahrzehnt von 2010 bis 2020 war weltweit das wärmste seit Beginn der Wetteraufzeichnungen, welche in Deutschland seit dem Jahr 1881 einheitlich durchgeführt werden. Auch die Verteilung des Niederschlages hat sich verändert und unterschied sich lokal sehr stark vom langjährigen Mittelwert.

Im Folgenden werden die Niederschlags- und Temperaturdaten der Jahre 2015 bis 2017 auf den Versuchsflächen in Brandenburg und Südniedersachsen (Göttingen) näher betrachtet. Als Referenzwert wurde der Bezugszeitraum von 1981 bis 2010 mit angeführt.

Innerhalb der beobachteten Vegetationszeiten gab es große Schwankungen bezüglich der Temperaturen (98 - $106 \%)$ und der Jahresniederschläge $(74-141 \%)$ um das langjährige Mittel herum (Tab. 5). Dabei konnte zumeist eine Frühjahrstrockenheit beobachtet werden, die sich in Bereichen zwischen $53-72 \%$ des normal zu erwartenden Niederschlages bewegte. 
Diese Trockenheit wurde dann von einzelnen, aber stark verregneten Monaten zur Mitte der Vegetationszeit abgelöst, wobei es sich um Niederschläge im Bereich zwischen 120 $-218 \%$ im Vergleich zum Referenzzeitraum handelt.

Nur in Brandenburg stellte das Frühjahr 2016 eine Ausnahme dar, indem es $21 \%$ mehr regnete als es sonst im langjährigen Mittel üblich gewesen wäre. Allerdings schloss sich dem ein sehr trockener Sommer an mit nur noch $50 \%$ des zu erwartenden Niederschlages.

Tab. 5: Vergleich der monatlichen Temperatur- und Niederschlagsentwicklung, der Jahre 2015 bis 2017 auf den Versuchsflächen in Brandenburg (BB) und Göttingen (Gö), mit dem langjährigen Mittelwert der Jahre 1981 - 2010. Quelle: eigene Darstellung auf Basis von DWD-Daten.

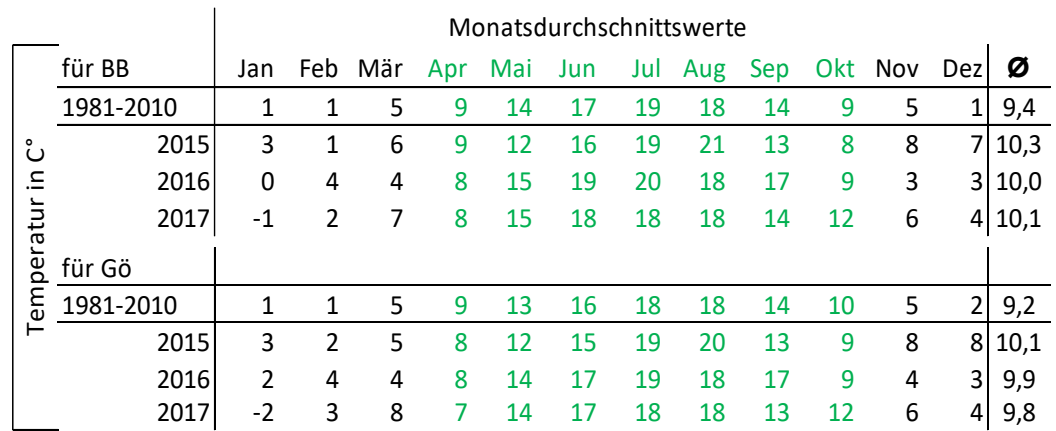

\begin{tabular}{rrr|c}
\multicolumn{3}{c|}{ Vegetationszeit in \% } & \\
Apr-Mai|Jun-Aug & Sep-Okt & $\boldsymbol{\varnothing}$ \\
\hline 100 & 100 & 100 & 100 \\
\hline 93 & 104 & 89 & 98 \\
102 & 106 & 111 & 106 \\
102 & 101 & 111 & 103 \\
& & & \\
\hline 100 & 100 & 100 & 100 \\
\hline 94 & 106 & 93 & 100 \\
102 & 106 & 111 & 106 \\
99 & 104 & 107 & 104
\end{tabular}

\begin{tabular}{|c|c|c|c|c|c|c|c|c|c|c|c|c|c|c|}
\hline \multirow{2}{*}{\multicolumn{2}{|c|}{$\frac{\text { für BB }}{1981-2010}$}} & & & & & & & & & & & & & \\
\hline & & 39 & 32 & 39 & 28 & 57 & 57 & 59 & 60 & 50 & 36 & 43 & 46 & 546 \\
\hline \multirow{8}{*}{ 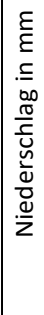 } & 2015 & 55 & 14 & 28 & 21 & 31 & 38 & 91 & 109 & 50 & 59 & 69 & 27 & 592 \\
\hline & 2016 & 34 & 44 & 27 & 20 & 83 & 36 & 29 & 23 & 24 & 44 & 26 & 33 & 422 \\
\hline & 2017 & 34 & 43 & 42 & 16 & 35 & 115 & 97 & 41 & 26 & 63 & 59 & 40 & 612 \\
\hline & für Gö & & & & & & & & & & & & & \\
\hline & $1981-2010$ & 52 & 39 & 52 & 41 & 65 & 66 & 66 & 58 & 56 & 46 & 54 & 56 & 651 \\
\hline & 2015 & 43 & 22 & 58 & 47 & 30 & 23 & 91 & 114 & 46 & 37 & 95 & 21 & 627 \\
\hline & 2016 & 42 & 47 & 32 & 28 & 41 & 113 & 42 & 41 & 35 & 72 & 34 & 16 & 544 \\
\hline & 2017 & 33 & 35 & 35 & 27 & 29 & 125 & 202 & 87 & 41 & 49 & 66 & 49 & 777 \\
\hline
\end{tabular}

\begin{tabular}{rrr|c} 
& & & \\
\hline 100 & 100 & 100 & 100 \\
\hline 62 & 135 & 126 & 115 \\
121 & 50 & 79 & 74 \\
60 & 144 & 104 & 113 \\
& & & \\
\hline 100 & 100 & 100 & 100 \\
\hline 72 & 120 & 82 & 97 \\
66 & 103 & 105 & 94 \\
53 & 218 & 87 & 141
\end{tabular}

Diese starken Klimaschwankungen haben negativen Einfluss auf die Überlebensrate der Jungpflanzen in ihrer kritischen Anwuchsphase genommen und erklären im Folgenden die hohen Ausfallraten.

Alle P.-Pflanzen wurden im Mai 2015 gepflanzt. Zum Ende der ersten Vegetationsperiode wurden sie, wie es für diese Art empfohlen wird, auf den Stock gesetzt, weshalb es sich bei dem Wachstum für 2016 um einen kompletten Neuaustrieb handelt.

Die Ausfallrate wurde ermittelt, indem alle Pflanzen gezählt wurden, die zu Beginn der nächsten Vegetationsperiode, also im Jahr darauf, wieder ausgetrieben sind.

In den Beobachtungsjahren sind unterschiedliche Anzahlen von Pflanzen ausgefallen, wobei sich die Verteilung stark zwischen den Vegetationsperioden und den beiden Versuchsstandorten unterscheidet. ${ }^{18}$

\footnotetext{
18 Die Versuchsfläche auf der Nordseeinsel Föhr stand zur Mitter der Vegetationsperiode 2015 für mehrere Wochen unter Wasser, weil die Gräben, die den Abfluss des Regenwassers ermöglichen sollten, nicht wie geplant von der zuständigen Gemeinde beräumt wurden. P.-Pflanzen vertragen Staunässe sehr schlecht und sterben schon nach drei Tagen ab.

Zu Beginn des Jahres 2016 waren keinerlei Pflanzen mehr auffindbar. Die Föhrer Versuchsfläche musste somit vorzeitig aufgegeben werden und findet daher keinerlei Berücksichtigung in der weiteren Ergebnisdarstellung.
} 


\subsubsection{Witterungsverhältnisse auf den Versuchsflächen}

Die hohen Ausfallraten auf den Versuchsflächen sind eine Folge der für die P.-Pflanzen ungünstigen Witterungsverhätlnisse.

Ausgelöst durch die geringen Niederschlagswerte des Frühjahres 2015 zeigt sich in Brandenburg bei einer Niederschlagsmenge von nur $62 \%$ eine fast doppelt so hohe Ausfallrate über alle Pflanzen hinweg, wie im Vergleich zu Göttingen mit $72 \%$ Niederschlag. Ein ähnlicher Effekt, nur von den Standorten her vertauscht, ist noch einmal für das Frühjahr 2017 zu beobachten mit knapp $60 \%$ Niederschlag in Brandenburg und nur $53 \%$ in Göttingen. Was die besonders hohe Ausfallrate in Göttingen in dieser Vegetationsperiode erklärt.

Wenn demzufolge das Frühjahr um ca. 30 \% trockener ausfällt, als es im langjährigen Mittel der Fall ist, so ist eine Ausfallrate der P.-Pflanzen im Bereich zwischen $20-30 \%$ zu beobachten (Abb. 19).

Fällt das Frühjahr hingegen um 40 \% trockener aus als sonst üblich, so schwankt die

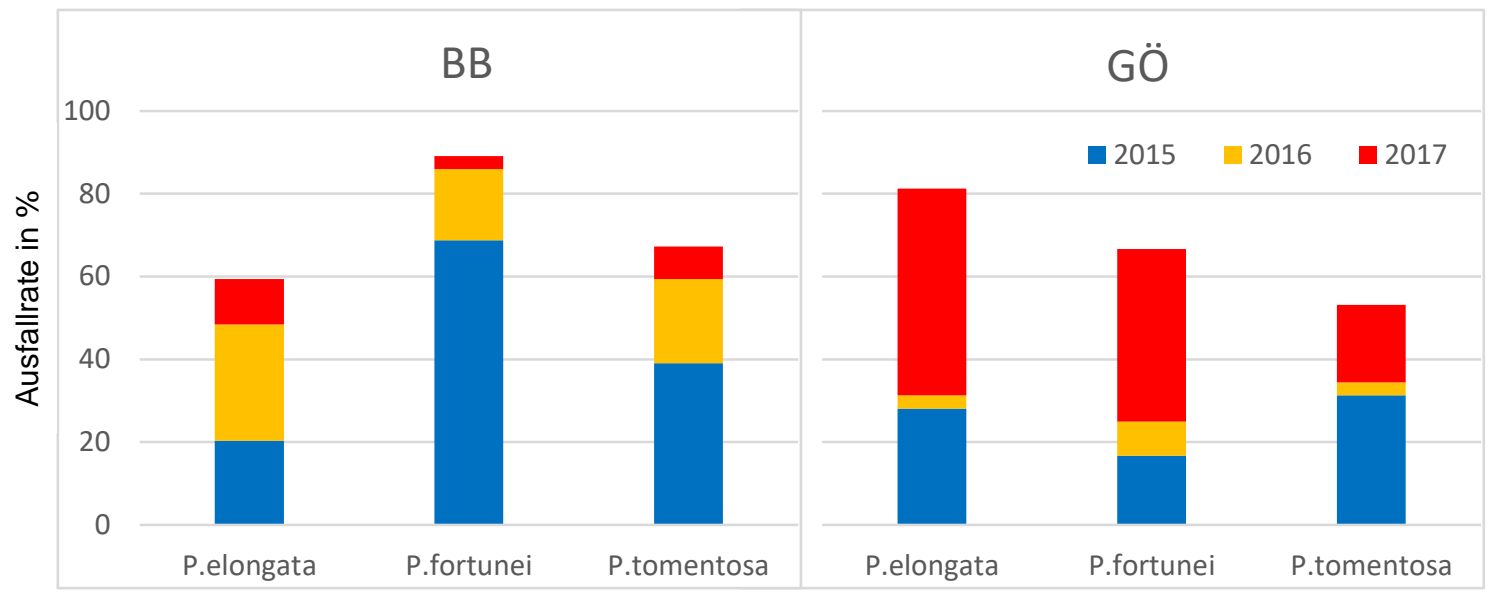

Abb. 19: Gesamt-Ausfallrate der Baumarten (in \%) auf den Versuchsflächen Brandenburg (BB) und Göttingen (GÖ), unterteilt in die Vegetationsperioden der Jahre 2015 bis 2017.

Ausfallrate in Abhängigkeit von der P.-Art um 20 - 70 \%.

Die Baumart P.elongata scheint hierbei die anfängliche Trockenheit des Jahres 2015 in Brandenburg am besten zu überstehen. In der Summe beider Standorte hat P.fortunei über alle drei Vegetationsperiode hinweg die höchsten Ausfälle zu verzeichnen. Am robustesten erscheint bei dieser Betrachtung hingegen P.tomentosa.

Selbst ein vergleichsweise niederschlagsreicher Sommer, in den Monaten von Juni bis August, wie er in den Jahren 2015 und 2017 auf beiden Versuchsstandorten mit Werten von $120-218 \%$ vorherrscht, kommt für viele P.-Pflanzen zu spät und kann die Trockenheit und den damit verbundenen Entwicklungsrückstand der ersten Vegetationsmonate, nicht mehr ausgleichen. Auch hier sei nochmal auf das sehr trockene Frühjahr 2017 in Göttingen verwiesen, wobei der gesamte Niederschlag der Vegetationszeit sich auf über $140 \%$ des üblichen Wertes summiert. Dieser Niederschlag konzentrierte sich aber ausschließlich auf die Sommermonate von Juni bis August und konnte von den P.-Pflanzen nicht mehr optimal genutzt werden.

Sowohl die Frühjahrs- als auch die Sommertrockenheit wurden noch zusätzlich durch die zumeist geringe Wasserspeicherkapazität der Böden auf den Versuchsflächen verschärft. Die Beschaffenheit der Böden ist zur leichteren Vergleichbarkeit in einer Überblickstabelle zusammengefasst (Tab. 6). 
Bei der Versuchsfläche in Brandenburg handelt es sich um einen ehemaligen Ackerstandort mit Braunerde-Podsol aus nährstoffarmen Sanden mit geringer Wasserspeicherkapazität und in Göttingen um eine anthropologisch (ehemalige Lagerfläche für Bauschutt) stark beanspruchte Rendzina aus Kalkgestein im Wechsel mit einer flachgründigen Braunerde.

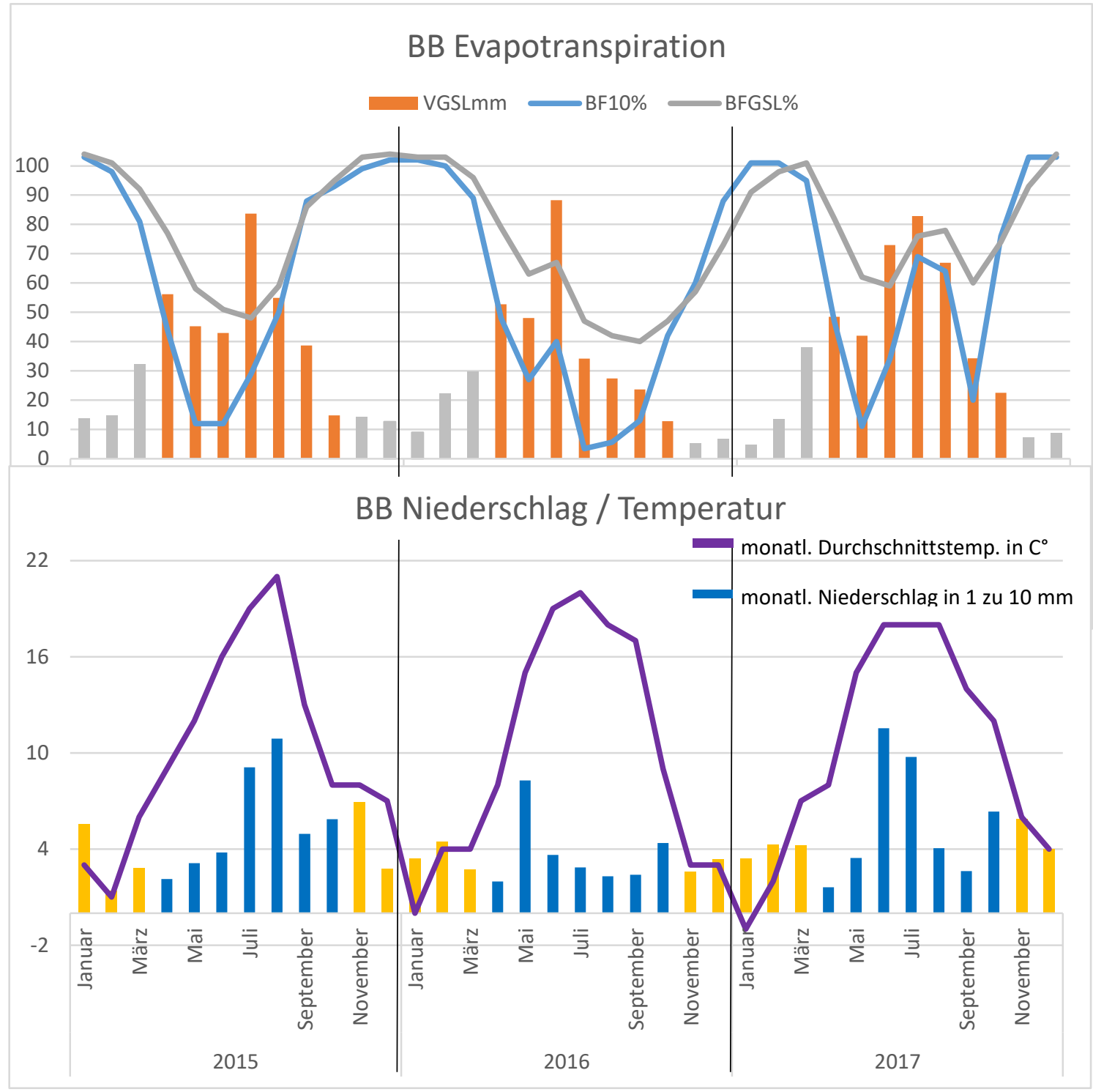

Abb. 20: Obere Grafik beschreibt die Evapotranspiration der Jahre 2015, 2016 und 2017 der Versuchsfläche Brandenburg (BB): VGSLmm = reale Evapotranspiration von Gras über sandigem Lehm (AMBAV), BF10\% = Bodenfeuchte unter Gras bei sandigem Lehm in einer Tiefe zwischen 0 $10 \mathrm{~cm}, \mathrm{BFGSL} \%$ = gibt die nutzbare Feldkapazität an und beschreibt die Bodenfeuchte unter Gras bei sandigem Lehm in einer Tiefe zwischen 0-60 cm; Untere Grafik: monatliche Niederschlagswerte der Versuchsfläche Brandenburg in $1 \mathrm{zu} 10 \mathrm{~mm}$ (blaue Balken = Vegetationsperiode), monatliche Durchschnittstemperatur in $C^{\circ}$ (violette Linie); Quelle: eigene Darstellung auf Basis von DWD-Daten.

Die Evapotranspiration ${ }^{19}$, in den Grafiken als VGSLmm mittels orangener Balken dargestellt, kennzeichnet den Wasserverbrauch der Pflanzen in den jeweiligen Monaten. Dieser Verbrauch ist umso höher je mehr Wasser durch Niederschläge den Pflanzen zur Verfügung stehen (Abb. 20 und Abb. 21).

19 Evapotranspiration (meteorologische Größe): bezeichnet die Summe aus direkter Verdunstung (von Boden- und Wasseroberflächen = Evaporation) und der Abgabe durch Pflanzen und Tiere (= Transpiration). 
Ergänzend dazu gibt die nutzbare Feldkapazität, in den Grafiken als BFGSL\%-Wert mittels der grauen Linie dargestellt, indirekt Aufschluss über den Trockenstress der Pflanzen.

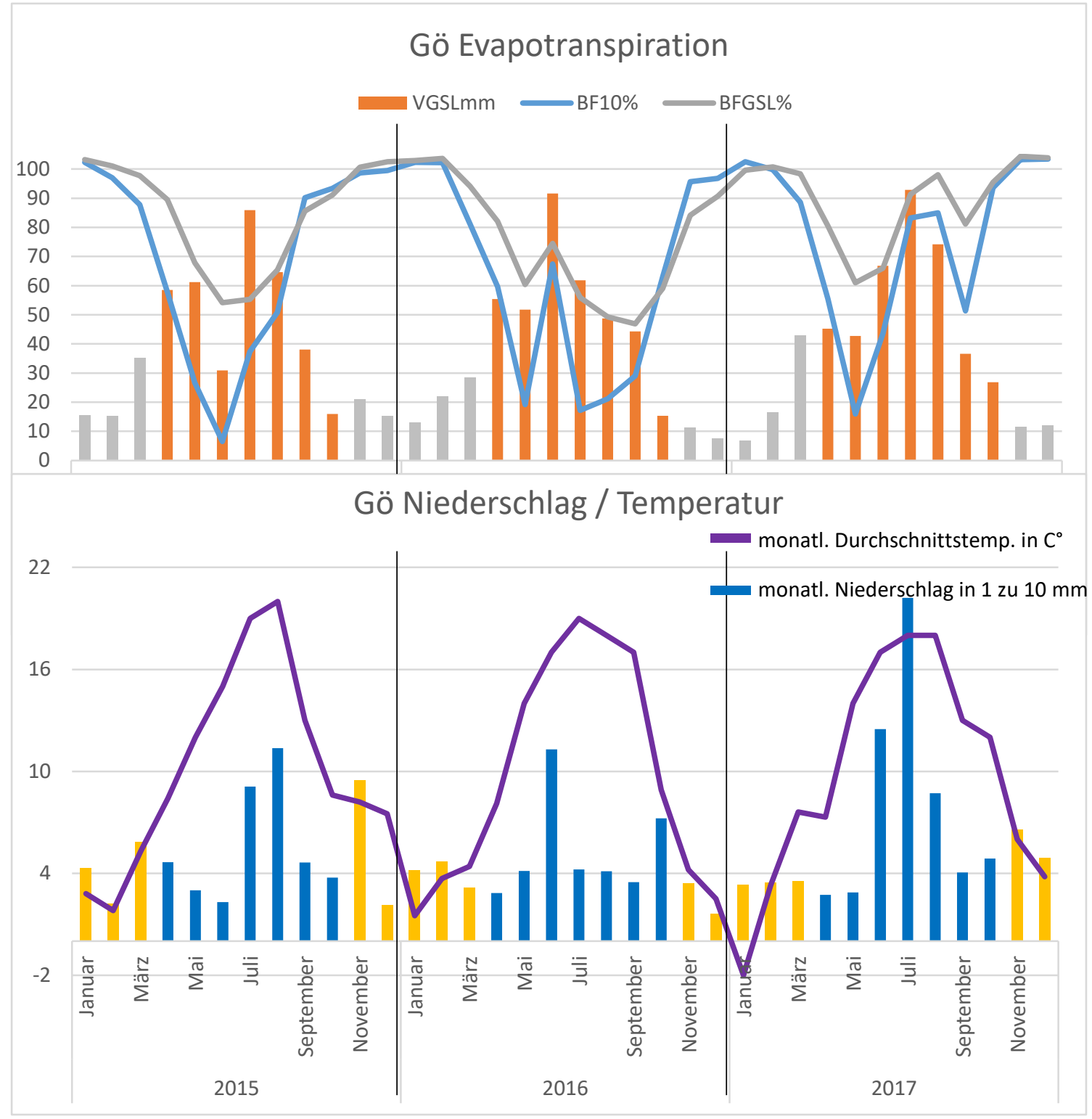

Abb. 21: Obere Grafik beschreibt die Evapotranspiration der Jahre 2015 bis 2017 der Versuchsfläche Göttingen (Gö): VGSLmm = reale Evapotranspiration von Gras über sandigem Lehm (AMBAV), BF10\% = Bodenfeuchte unter Gras bei sandigem Lehm in einer Tiefe zwischen $0-10 \mathrm{~cm}$, BFGSL\% = gibt die nutzbare Feldkapazität an und beschreibt die Bodenfeuchte unter Gras bei sandigem Lehm in einer Tiefe zwischen 0-60 cm; Untere Grafik: monatliche Niederschlagswerte der Versuchsfläche Göttingen in $1 \mathrm{zu} 10 \mathrm{~mm}$ (blaue Balken = Vegetationsperiode), monatliche Durchschnittstemperatur in $\mathrm{C}^{\circ}$ (violette Linie); Quelle: eigene Darstellung auf Basis von DWD-Daten.

Die nutzbare Feldkapazität ${ }^{20}$ des effektiven Wurzelraumes ist ein wichtiges Kriterium zur Beurteilung des Wasserhaushaltes eines Standortes. Über diesen Wert wird die Kapazität eines Bodens beschrieben, Wasser pflanzenverfügbar zu speichern.

20 Die nutzbare Feldkapazität des effektiven Wurzelraumes ist das Produkt aus der nutzbaren Feldkapazität (zumeist als $\mathrm{nFK}$ in mm bzw. dm angegeben) und der effektiven Durchwurzelungstiefe. 
Bei dem in den Abb. 20Abb. 21 angegebenen BFGSL\%-Wert handelt es sich nur um einen Durchschnittswert in der Bodentiefe von 0-60 cm. Der BF10\%-Wert (blaue Linie) hingegen ist als Angabe der Bodenfeuchte, im Bereich zwischen $0-10 \mathrm{~cm}$, beim Anwuchs der Pflanzen aussagekräftiger, da diese zu Beginn noch über kein ausgeprägtes Wurzelsystem verfügen und von der Feuchte im Oberboden abhängig sind.

Da die nutzbare Feldkapazität immer zuerst im Oberboden erschöpft ist, lassen sich aus der Differenz zwischen dem BFGSL\%- und dem BF10\%-Wert die Monate mit geringer Wasserverfügbarkeit herauslesen.

Die Jungpflanzen haben, aufgrund des sich noch zu entwickelnden Wurzelsystems, keinen Anschluss an das Grundwasser und können somit nur das Wasser verbrauchen, welches im Boden verfügbar ist und durch die unregelmäßigen Niederschlagsereignisse wieder aufgefüllt wird. Wenn durch einen länger anhaltenden Mangel an Niederschlag die nutzbare Feldkapazität in den Böden erschöpft ist, leiden die Jungpflanzen unter Trockenstress.

Die Ausfallrate in Brandenburg ist in der Vegetationsperiode 2015 deutlich höher als in Göttingen, was ein Resultat der enormen Trockenheit der Monate Mai und Juni ist, wie sich anhand dem BF10\%-Wert erkennen lässt, der für den Zeitraum bei knapp über 10 \% liegt. Im Vergleich dazu wird in Göttingen nur im Monat Juni 2015 eine solche Trockenheit erreicht, wobei die Bodenfeuchte hier bei einer Tiefe zwischen $0-10 \mathrm{~cm}$ in Bereiche von unter $10 \%$ und zwischen $0-60 \mathrm{~cm}$ Tiefe auf ca. $50 \%$ absinkt. Dies erklärt die etwas geringere Ausfallrate in Göttingen.

In den trockenen Sommermonaten des Jahres 2016 werden Extremwerte erreicht und die nutzbare Feldkapazität (0 - $60 \mathrm{~cm}$ Bodentiefe) sinkt auf der Versuchsfläche in Brandenburg auf unter $40 \%$ und die Bodenfeuchte im Bereich zwischen $0-10 \mathrm{~cm}$ Tiefe verringert sich auf unter $5 \%$.

Der sich daraus ergebende Trockenstress schränkt besonders zu Beginn der Vegetationszeit das Pflanzenwachstum stark ein, indem er den Austrieb verzögert. Der daraus resultierende Zuwachsverlust kann im Laufe des Sommers nicht wieder ausgeglichen werden und führt indirekt zu einer Schwächung der Pflanze, die es nicht vermag, ein großes Wurzelsystem auszubilden und ausreichend Reservestoffe für das nächste Frühjahr einzulagern. Wenn sich dieser Kreislauf mehrere Vegetationsperioden hintereinander wiederholt, dann führt diese andauernde Schwächung unweigerlich zu einem Absterben der Pflanze.

Im Verlauf der Vegetationsperiode 2018 sind nahezu alle noch verbliebenen Pflanzen auf den Flächen in Brandenburg und Göttingen abgestorben. Nur in Brandenburg haben ca. $10 \%$ der P.-Pflanzen die ersten drei Jahre des Anwuchses überstanden und sind zu jungen Bäumen herangewachsen. Sie liegen aber in ihrem natürlichen Höhenwachstum weit hinter den Erwartungen zurück.

Aufgrund der unerwartet hohen Ausfallraten der Pflanzen kam es nur zu einer vergleichsweise geringen Datenausbeute. Alle Beobachtungen wurden mit der Vegetationsperiode 2017 vorzeitig eingestellt. Zur weiteren Analyse des Wuchsverhaltens der Gattung Paulownia unter mitteleuropäischen Bedingungen stehen folglich nur die Daten aus den drei Vegetationsperioden 2015 bis 2017 zu Verfügung. 
Tab. 6: Tabellarischer Überblick über die Bodenbeschaffenheit auf den Versuchsflächen in Brandenburg, Föhr und Göttingen. Quelle: eigene Darstellung mittels Geoviewer und DWD-Daten.

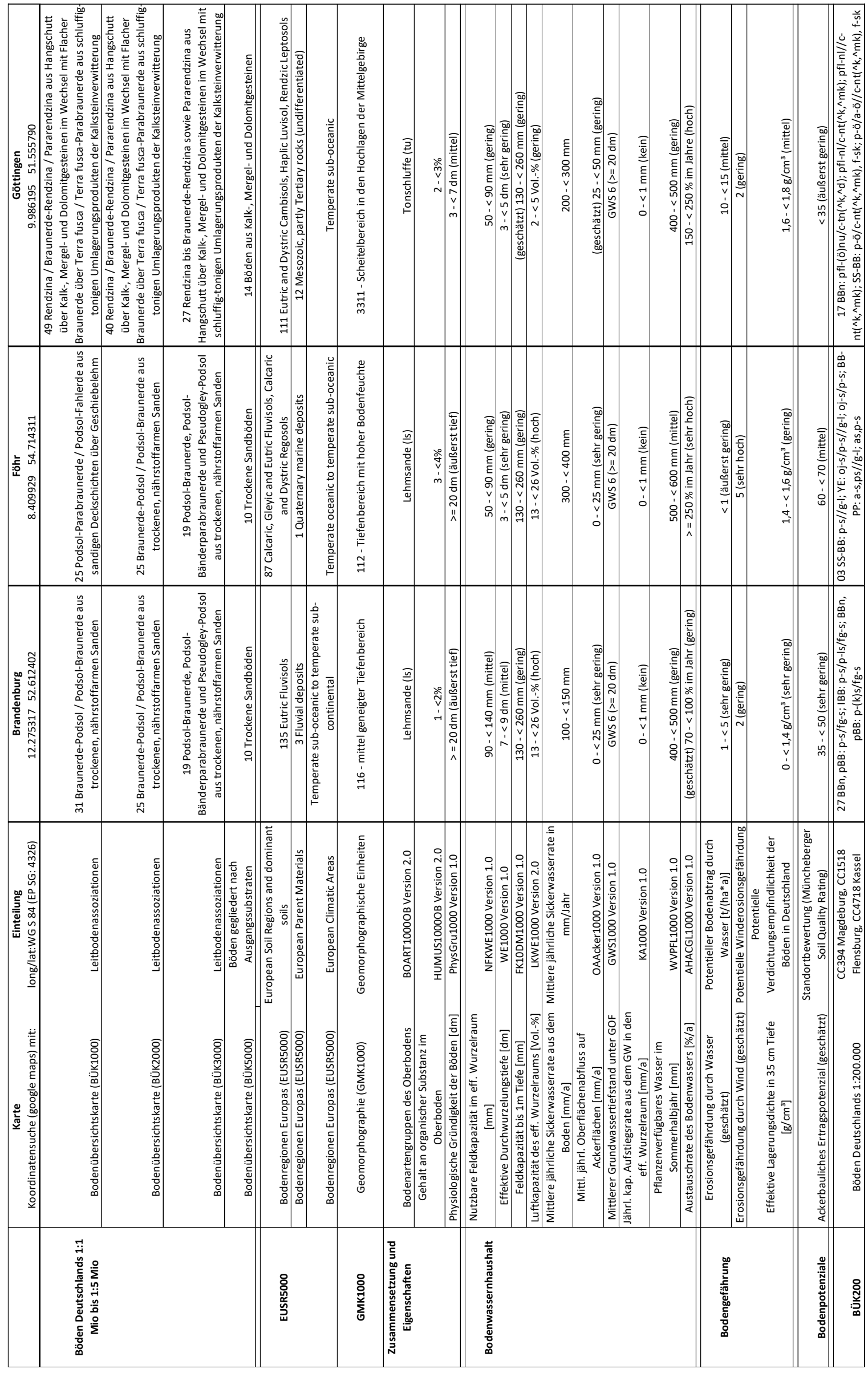




\subsubsection{Jugendwachstum im Freiland}

Die Ausfallrate beschreibt nur einen Teilaspekt zum Wachstum der Pflanzen in der Kulturphase. Deutlich aussagekräftiger ist ein Vergleich des Höhenwachstums der Arten untereinander.

Das jährliche Höhenwachstum ist die Summe aus den genetischen Eigenschaften des Pflanzentyps, den vorherrschenden Standorteigenschaften, wie Bodentyp und Klimaverhältnisse (Niederschlag, Temperatur, usw.), plus dem Einfluss durch unterschiedliche Behandlungsarten (N, G, M, GM).

Welche dieser Variablen Einfluss auf das Höhenwachstum genommen haben, wurde statistisch getestet (multifaktorielle Varianzanalysen (ANOVA)) und wird im Folgenden beschrieben.

\subsection{Jugendwachstum nach Behandlungsart}

In der Abb. 22 ist mittels mehrerer Box-Plots eine vergleichende Darstellung des Wuchsverhaltens aller P.-Pflanzen zwischen den Versuchsflächen in Brandenburg (blau) und Göttingen (grün) vorgenommen worden, unterteilt nach den Vegetationsperioden 2015, 2016 und 2017 und aufgeteilt nach den verschiedenen Behandlungsarten (N, G, M, GM):

$\mathrm{N} \quad=$ unbehandelt (Nullfläche) ohne Zusätze von Bodenhilfsstoffen,

$\mathrm{G} \quad=$ Geohumus, Zugabe von $100 \mathrm{ml}$ Geohumusgranulat in das Pflanzloch

$\mathrm{M} \quad=$ Mykorrhiza, Zugabe von $100 \mathrm{ml}$ Mykorrhizapilz-Konzentrat

$\mathrm{GM}=$ Zugabe von $100 \mathrm{ml}$ Geohumusgranulat und $100 \mathrm{ml}$ Mykorrhizapilz-Konzentrat.

Auf ein und derselben Versuchsfläche kann, im Verlauf der drei Vegetationsperioden, zwischen den Behandlungsgruppen kein signifikanter ${ }^{21}$ Unterschied, also folglich kein Einfluss auf das Höhenwachstum, festgestellt werden (Tab. 13).

Auch die Mykorrhizierung und der Bodenhilfsstoff Geohumus hatten keinen nachweislichen $^{22}$ Einfluss auf das Höhenwachstum der P.-Pflanzen. Folglich unterscheiden sich die vier Behandlungsarten in den ersten drei Vegetationsperioden nicht signifikant voneinander.

Der einzige statistische nachweisbare und in den Grafiken auch optisch erkennbare Unterschied, besteht zwischen den Standorten Brandenburg und Göttingen.

Die Standorteigenschaften haben folglich einen signifikanten Unterschied im Höhenwachstum der P.-Pflanzen bewirkt (Abb. 22).

21 Durch mehrere zweifaktorielle Varianzanalysen wurde der mögliche Einfluss der verschiedenen Behandlungsarten auf das Höhenwachstum der P.-Pflanzen getestet. Es zeigt sich dabei kein signifikanter Einfluss der Behandlungsarten auf das Höhenwachstum.

22 Nach einem F-Test (Levene-Test) zeigen die Behandlungsgruppen (N, G, M, GM) unterschiedliche Varianzen. Der darauf angewandte Games-Howell Post-hoc-Test, der als Voraussetzungen ungleiche Stichprobenumfänge (unterschiedliche hohe Ausfallraten der Pflanzen) und ungleiche Varianzen zulässt, zeigt keinerlei Signifikanzen zwischen den vier Behandlungsgruppen, ganz gleich welche Vegetationsperiode getestet wurde. 
Die Spannweiten der Brandenburger Höhen fallen im Vergleich zu den Göttingern deutlich größer aus. Dies ist eine Folge dessen, dass es in Brandenburg sowohl sehr große als auch sehr kleine Pflanzen gibt.

Für die Vegetationsperiode 2015 ist noch kein signifikanter Unterschied zwischen den Standorten zu beobachten. Er lässt sich erst ab der Vegetationsperiode 2016 nachweisen und dass, obwohl es sich beim Höhenwachstum 2016 um einen vollständigen Neuaustrieb der Pflanzen handelt, nachdem diese Ende 2015 auf den Stock gesetzt wurden.

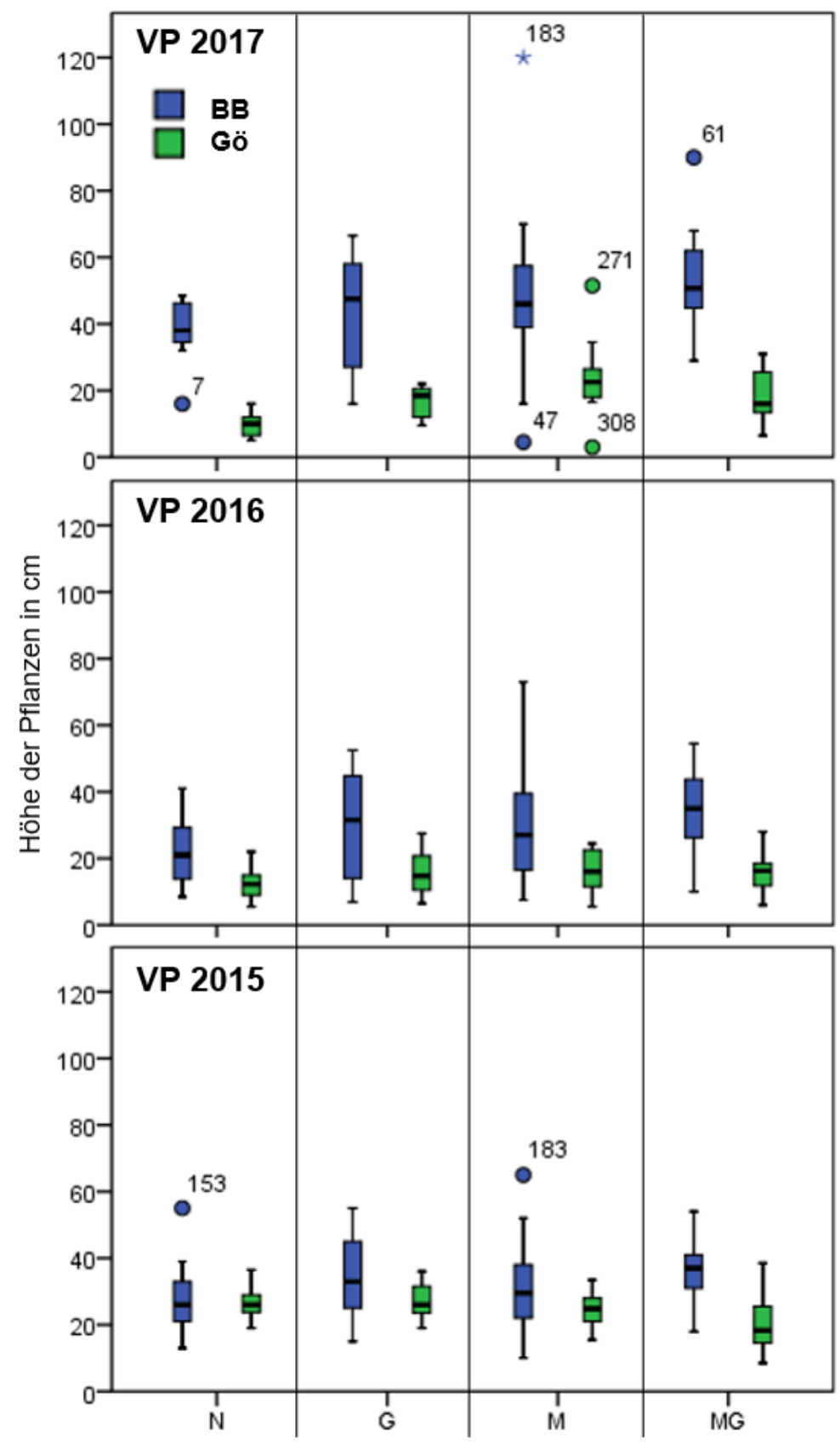

Abb. 22: Höhenwachstum der Paulownia-Pflanzen (in $\mathrm{cm}$ ) für die Vegetationsperioden (VP) 2015, 2016 und 2017, für die Versuchsflächen Brandenburg (BB) und Göttingen (Gö); unterteilt nach Behandlungsarten: $N=$ unbehandelt (Nullfläche), $\mathbf{G}=$ Geohumus, $\mathbf{M}=$ Mykorrhizierung, $\mathrm{GM}=$ Geohumus + Mykorrhizierung.

\subsubsection{Unterschiede im Jugendwachstum der P.-Arten}

Auch wenn sich für die Behandlungsarten kein Einfluss auf das Höhenwachstum der Pflanzen innerhalb der ersten drei Vegetationsperioden nachweisen lässt, so könnten durchaus Unterschiede zwischen den drei P.-Arten vorhanden sein. Daher werden im Folgenden die Behandlungsarten pro Baumart näher betrachtet. 


\subsection{Brandenburger Versuchsfläche}

Wie in der Abb. 23, anhand der nur als Striche angedeuteten Box-Plots, erkennbar ist, sorgen die hohen Ausfallraten der Pflanzen, bereits ab der zweiten Vegetationsperiode (2016), für eine eingeschränkte Datengrundlage, was die Aussagekraft vermindert.

Auch die doppelte Anzahl an Plots und somit auch an Pflanzenanzahl, wie es in Brandenburg im Verhältnis zur Göttinger Versuchsfläche der Fall ist, kann diesen Verlust an Daten nicht ausgleichen.
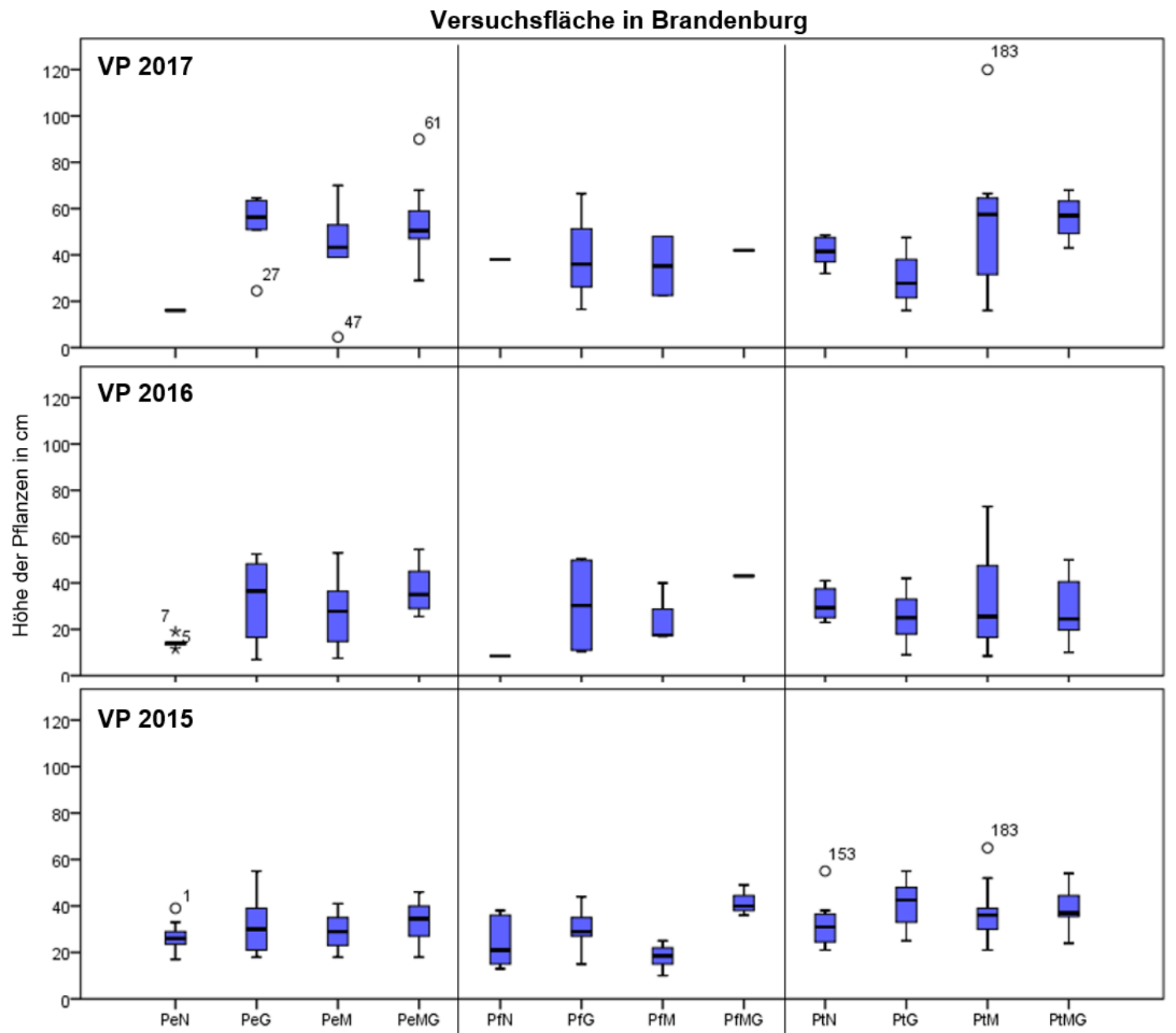

Abb. 23: Höhenwachstum der Paulownia-Arten (in $\mathrm{cm}$ ) auf der Versuchsfläche in Brandenburg; Pe = P.elongata $; \mathrm{Pf}=$ P.fortunei; $\mathrm{Pt}=$ P.tomentosa für die Vegetationsperioden (VP) 2015, 2016 und 2017 unterteilt nach Behandlungsarten: $N=$ unbehandelt (Nullfläche), $\mathbf{G}=$ Geohumus, $M=$ Mykorrhizierung, GM = Geohumus + Mykorrhizierung.

Hervorzuheben ist die von Vegetatoinsperiode zu Vegetationsperiode größer werdende Spannweite beim Höhenwachstum (Abb. 23). Sie ergibt sich dadurch, dass einzelne Pflanzenindividuen zum Ende der Vegetationszeit verholzen und im nächsten Frühjahr weiterwachsen und somit größer werden und andere Pflanzen immer wieder zu Beginn der Vegetationsperiode vom Boden neuaustreiben.

Die Pflanzenindividuen sind auf der Brandenburger Versuchsfläche, bezogen auf ihr Höhenwachstum, sehr heterogen. Die aus diesen Werten resultierende Spannweite beeinflusst maßgeblich die signifikanten Unterschiede im Vergleich zur Göttinger Versuchsfläche. 


\subsection{Göttinger Versuchsfläche}

Beim Höhenwachstum der P.-Arten ist bei einem Vergleich der Vegetationsperiode von 2015 zu 2016, anders als es in Brandenburg der Fall ist, in Göttingen ein Rückgang der Höhen erkennbar. Dieser ist eine Folge des Neuaustriebes der überlebenden P.Pflanzen in der Vegetationsperiode 2016, da diese zum Ende der Vegetationsperiode 2015, wie für diese Art üblich, auf den Stock gesetzt wurden (Abb. 24).

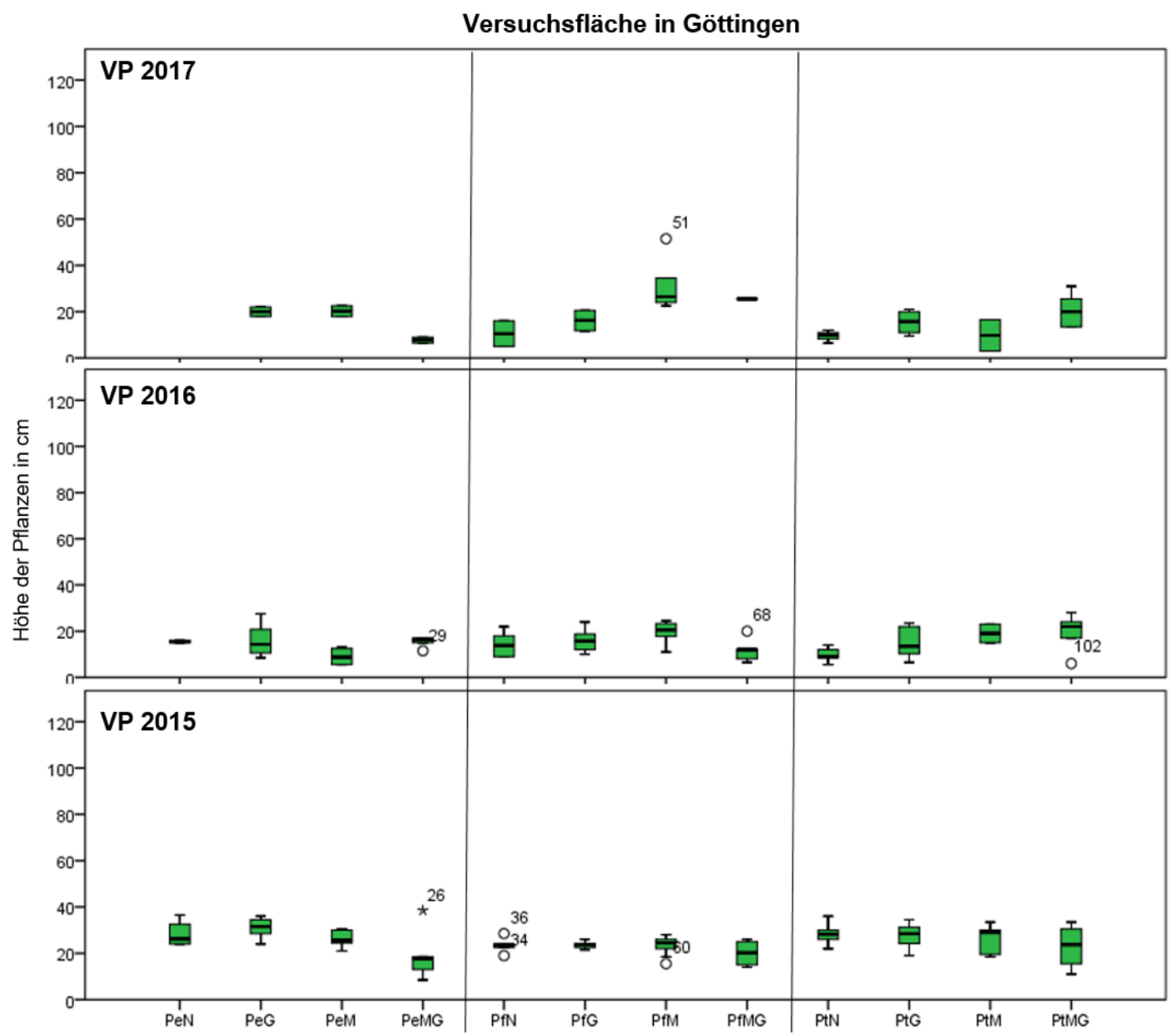

Abb. 24: Höhenwachstum der Paulownia-Arten (in $\mathrm{cm}$ ) auf der Versuchsfläche in Göttingen; Pe = P.elongata; Pf = P.fortunei; Pt = P.tomentosa für die Vegetationsperioden (VP) 2015, 2016 und 2017 unterteilt nach Behandlungsarten: $N=$ unbehandelt (Nullfläche), $G=$ Geohumus, $M=$ Mykorrhizierung, GM = Geohumus + Mykorrhizierung.

Der Neuastrieb, direkt vom Boden aus, schwächt die Pflanzen. Er geht mit einer anfänglichen nur geringen Blattmasse einher. Im Vergleich zu schon verholzten Pflanzen brauchen die Pflanzen mit dem Neuaustreib mehrere Wochen, um den Rückstand an Höhewachstum auszugleichen. Durch die anfänglich verminderte photosynthetische Leistungsfähigkeit, eine direkte Folge der geringeren Blattmasse, bilden die Pflanzen auch weniger Reservestoffe, da sie ihre gesamte Energie in das Längenwachstum investieren. Sie werden somit von Jahr zu Jahr schwächer und treiben als Folge nicht mehr neu aus.

Die fehlenden Werte in der Grafik, wie z.B. die Lücke bei PeN in der Vegetationsperiode 2017 (Abb. 24) sind eine Folge der hohen Ausfallrate auf der Göttinger Versuchsfläche. 
Einzelne Signifikanzen sind zwischen den Baumarten und den verschiedenen Behandlungsarten sowohl in Brandenburg als auch in Göttingen nachweisbar, wie die Tab. 13 darstellt (siehe Anhang Kapitel III).

Anhand dieser lassen sich aber keine Tendenzen ableiten.

Es lässt sich aber auch nicht ausschließen, dass sich Unterschiede zwischen den Behandlungsarten und den Baumarten erst in späteren Vegetationsperioden herausbilden können. Hierfür wäre aber eine größere Versuchsanordnung mit längerem Beobachtungszeiträumen nötig.

\subsubsection{Zum Anbau im Gewächshaus}

Um den Einfluss unterschiedlicher Belichtung und Bewässerung auf das Jugendwachstum von P.-Pflanzen untersuchen zu können, wurde ein Experiment im Gewächshaus konzipiert, welches zwei Vegetationsperioden umfasst.

Hierbei wurde die Wechselwirkung von drei verschiedenen Belichtungs- (100 \%, $60 \%$, $40 \%$ Licht) und Bewässerungsstufen (100\%, $60 \%, 40 \%$ Wasser) auf die drei P.-Arten P.elongata, P.fortunei und P.tomentosa beobachtet. Die sich daraus ergebenden 27 verschiedenen Behandlungsvarianten (3 Pflanzenarten $\times 3$ Belichtungsstufen $\times 3$ Bewässerungsstufen $=27$ ) haben jeweils einen Stichprobenumfang von 20 Pflanzen, was die Gesamtanzahl auf 540 Pflanzen erhöht.

Die Pflanzen entspringen in der ersten Vegetationsperiode alle als Keimlinge dem Boden und verbrachten die ersten 4 Wochen in der Baumschule. Anschließend wurden sie gleichmäßig auf die jeweiligen Belichtungs- und Bewässerungsstufen aufgeteilt. Alle Pflanzen wurden sowohl am Ende der ersten als auch der zweiten Vegetationsperiode auf den Stock gesetzt, um die oberirdische Biomasse analysieren zu können. Folglich repräsentiert das Wachstum der zweiten Vegetationsperiode einen kompletten Neuaustrieb.

In einem ersten Analyse-Schritt werden alle Beobachtungen auf Gattungsebene und mit einem Vergleich der Vegetationsperioden untereinander beschrieben. Dabei bauen die Behandlungsarten (Licht und Wasser) und -stufen (100\%, $60 \%, 40 \%)$ in der Ergebnisdarstellung ergänzend aufeinander auf.

Erst in einem zweiten Schritt wird der Wachstumsverlauf der drei P.-Arten untereinander verglichen (3.2.2.3 Jugendwachstum bei einem Vergleich der Arten).

Der Einfluss einer unterschiedlichen Belichtung und Bewässerung auf den Wachstumsverlauf lässt sich durch das Längen- und des Durchmesserwachstum der ersten und zweiten Vegetationsperiode beschreiben. Darauf aufbauend lassen sich auch über die gewachsene oberirdische Biomasse Rückschlüsse auf das Wachstum der Pflanzen schließen. Die Begrenzung des Lichtes und des Wassers haben einen negativen Einfluss auf die Überlebensrate der Jungpflanzen genommen und erklären im Folgenden die hohen Ausfallraten. 


\subsubsection{Ausfallrate im Gewächshaus}

Im Verlauf der zwei untersuchten Vegetationsperioden kam es bei allen Behandlungsstufen zu Ausfällen, mit Ausnahme der 100\% Licht- zu 60\% Wasserstufe der ersten Vegetationsperiode, wie die Tab. 7 verdeutlicht.

Bereits in der ersten Vegetationsperiode ist mit der weiteren Verringerung der Lichtzufuhr (in der Tabelle von links nach rechts) im Durchschnitt eine stetige Zunahme der Pflanzenausfälle in den Bereichen zwischen $2-10 \%$, über alle Wasserstufen hinweg, erkennbar. Dieser Effekt verstärkt sich in der zweiten Vegetationsperiode auf eine Ausfallrate in einem Bereich von 7 - $49 \%$ weiter.

Eine Abnahme des Wassers (in der Tabelle von oben nach unten) hat anfänglich einen ähnlich schwachen Effekt auf die Ausfallrate in Höhe von $5-7 \%$. Erst in der zweiten Vegetationsperiode grenzt sich die $40 \%$ Wasserstufe deutlich zu der $60 \%$ und $100 \%$ Stufe ab und erreicht Ausfallraten von $15-61 \%$, in Abhängigkeit von der Lichtstufe.

Im Durchschnitt ist bei einer Abnahme der Wassermenge eine Erhöhung der Ausfallrate um $16-26 \%$, über alle Lichtstufen hinweg, erkennbar.

In der ersten Vegetationsperiode ergibt sich der höchste Ausfall an Pflanzen, mit einem Wert von über $11 \%$, bei der 40 $\%$ Licht- und $100 \%$ Wasserstufe.

Die Baumart P.elongata beeinflusst hier mit $30 \%$ Ausfall die Höhe dieses Wertes maßgeblich.

Den geringsten Ausfall über alle Behandlungsstufen hinweg hat mit $3 \%$ die P.tomentosa.

Im Gegensatz dazu hat P.elongata eine mehr als doppelt so hohe durchschnittliche Ausfallrate von $8 \%$.

Tab. 7: Ausfallrate der Paulownia-Pflanzen (in \%) aufgeschlüsselt nach Behandlungsstufen (Licht und Wasser) und unterteilt in erste und zweite Vegetationsperiode und nach Arten (P.elongata $(\mathrm{Pe})$, P.fortunei (Pf), P.tomentosa (Pt)).

\begin{tabular}{|c|c|c|c|c|c|c|c|c|c|c|c|c|}
\hline & & & & & eget & tion & peri & de & & & & \\
\hline & & & OLic & & & OLich & & & OLich & & & \\
\hline & $\%$ & $\mathrm{Pe}$ & $\mathrm{Pf}$ & $\mathrm{Pt}$ & $\mathrm{Pe}$ & $\mathrm{Pf}$ & $\mathrm{Pt}$ & $\mathrm{Pe}$ & Pf & $\mathrm{Pt}$ & $\varnothing$ & \\
\hline$\pi$ & $\mathrm{Pe}$ & 10 & & & 5 & & & 30 & & & 15,0 & \\
\hline క & $\mathrm{Pf}$ & & 0 & & & 10 & & & 0 & & 3,3 & $\stackrel{\infty}{N}$ \\
\hline & $\mathrm{Pt}$ & & & 0 & & & 10 & & & 5 & 5,0 & \\
\hline & $\varnothing$ & & 3,3 & & & 8,3 & & & 11,7 & & & \\
\hline$\sigma$ & $\mathrm{Pe}$ & 0 & & & 0 & & & 10 & & & 3,3 & \\
\hline 3े & $\mathrm{Pf}$ & & 0 & & & 15 & & & 15 & & 10,0 & 오 \\
\hline & $\mathrm{Pt}$ & & & 0 & & & 0 & & & 5 & 1,7 & \\
\hline & $\varnothing$ & & 0 & & & 5 & & & 10 & & & \\
\hline$\pi$ & $\mathrm{Pe}$ & 10 & & & 10 & & & 10 & & & 10,0 & \\
\hline 호 & Pf & & 5 & & & 5 & & & 5 & & 5,0 & है \\
\hline & $\mathrm{Pt}$ & & & 0 & & & 0 & & & 15 & 5,0 & \\
\hline & $\varnothing$ & & 5 & & & 5 & & & 10 & & & \\
\hline & $\varnothing$ & 4,7 & 1,7 & 0 & 5,7 & 10 & 3,3 & 14,3 & 6,7 & 8,3 & & \\
\hline & & & 2,8 & & & 6,1 & & & 10,6 & & & \\
\hline
\end{tabular}

2. Vegetationsperiode

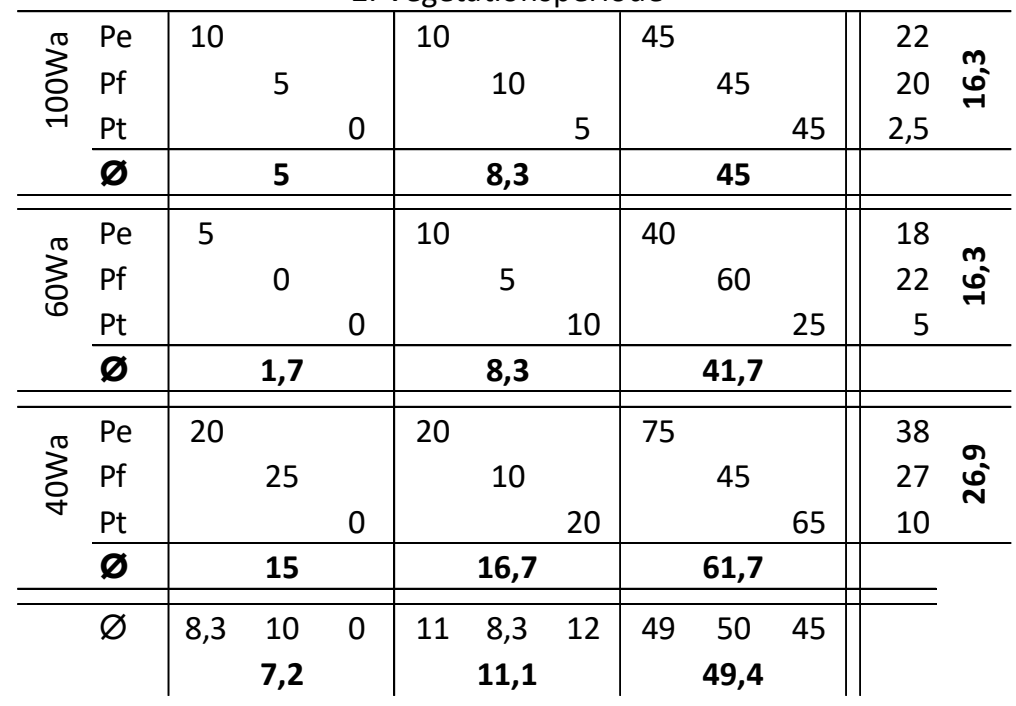


Bei einem Vergleich der Wasserstufen über alle Lichtstufen hinweg ergeben sich durchschnittliche Ausfallzahlen von 5-7\% und nur eine geringe Spannweite. Wobei die $100 \%$ Wasserstufe mit $7 \%$ die höchste Ausfallrate zu verzeichnen hat.

Anders stellt es sich da bei einem Vergleich der Lichtstufen über alle Wasserstufen hinweg. Hierbei ergeben sich Ausfallraten von 2-10\%. Es ist daher davon auszugehen, dass die geringere Lichtzufuhr einen größeren Einfluss auf die Ausfallraten der Baumarten genommen hat als die Wassermenge.

In der zweiten Vegetationsperiode zeigt die $40 \%$ Licht- zu $40 \%$ Wasserstufe mit einem Wert von $61 \%$ die höchste Ausfallrate.

Ein Vergleich der Baumarten in dieser Behandlungsstufe zeigt eine Spanne der Ausfallraten von $45-75 \%$.

Mit durchschnittlich $12 \%$ hat sich die Ausfallrate bei P.tomentosa, über alle Behandlungsstufen hinweg, im Kontrast zur ersten Vegetationsperiode verdreifacht. Trotz dessen zeigt P.tomentosa im Vergleich zu den anderen Baumarten noch immer die geringste Ausfallrate.

Den Gegensatz dazu bildet auch hier wieder P.elongata mit einer durchschnittlich doppelt so hohen Ausfallrate von $24 \%$.

Die Pflanzen-Ausfälle schwanken bei einem Vergleich der Wasserstufen über alle Lichtstufen hinweg von $16-26 \%$. Wobei in der zweiten Vegetationsperiode im Gegensatz zur ersten nun die $40 \%$ Wasserstufe die höchsten Ausfälle zu verzeichnen hat.

Eine größere Spannweite ergibt sich hingegen bei Betrachtung der Lichtstufen über alle Wasserstufen hinweg. Es zeigen sich hierbei Ausfallraten von $7-49 \%$.

Im Allgemeinen ist zwischen der 100er zur 60er Stufe, ganz gleich ob es sich dabei um Wasser oder Licht handelt, nur ein geringer Pflanzenausfall zu beobachten, der $8 \%$ nicht überschreitet. Erst eine weitere Verringerung, sowohl des Lichtes als auch des Wassers auf $40 \%$, erhöht die Ausfallrate deutlich auf einen Wert von bis zu $61 \%$.

In der ersten Vegetationsperiode hat sich der größere Einfluss des Lichtes im Vergleich zum Wasser auf die Pflanzenausfällte bereits abgezeichnet, und dieser Einfluss kann verstärkt auch in der zweiten Vegetationsperiode beobachtet werden.

Zwischen der $100 \%$ (7,2\%) zu $60 \%(11,1 \%)$ Lichtstufe erhöht sich die Ausfallrate um den Faktor 1,5. Eine deutlichere Zunahme ist jedoch zwischen der Lichtstufe von $60 \%$ $(11,1 \%)$ zu $40 \%(49,4 \%)$ zu beobachten. Hier verstärkt sich der Faktor auf über 4,4. Generell ist bei $40 \%$ Licht ein Ausfall von über $41 \%$ der Pflanzen zu verzeichnen, und dabei ist es unerheblich wie viel Wasser zur Verfügung steht.

\subsubsection{Jugendwachstum auf Gattungsebene}

Die Ausfallraten der verschiedenen Behandlungsstufen nehmen indirekten Einfluss auf die Ergebnisdarstellung, weil sich vor allem durch das Absterben der geringwüchsigen Pflanzen bzw. in Folge der dann kleineren Stichproben Mittelwerte verschieben. 
Auch Ausreißer, die in den Boxplot-Darstellungen einzeln als Nummerierungen zumeist am Rande der Abbildungen aufgeführt werden, dürfen nicht unerwähnt bleiben. Sie sind am häufigsten in der zweiten Vegetationsperiode zu finden und geben darüber Aufschluss, wie sich die tatsächliche Verteilung der Pflanzenhöhen, Durchmesser oder Trockenmassen darstellt. Besonders hervorzuheben sind sie im Bereich der $40 \%$ Lichtstufe über alle Pflanzenarten hinweg.

Um die eben genannten Schwierigkeiten zu umgehen, wurden in einem ersten Schritt alle Pflanzen nach Behandlungsarten (Licht und Wasser) und -stufen (100\%, $60 \%, 40$ $\%)$ unterteilt, ganz gleich welcher P.-Art sie entstammen. Dadurch erhöht sich der allgemeine Stichprobenumfang auf 60 Pflanzen pro dargestellten Boxplot, abzüglich der jeweiligen Ausfälle.

\subsection{Längenwachstum}

Ein Vergleich der verschiedenen Behandlungsstufen innerhalb der ersten Vegetationsperiode (in der Abb. 25 blau) zeigt keinen signifikanten Unterscheid in der Höhenentwicklung der P.-Pflanzen.
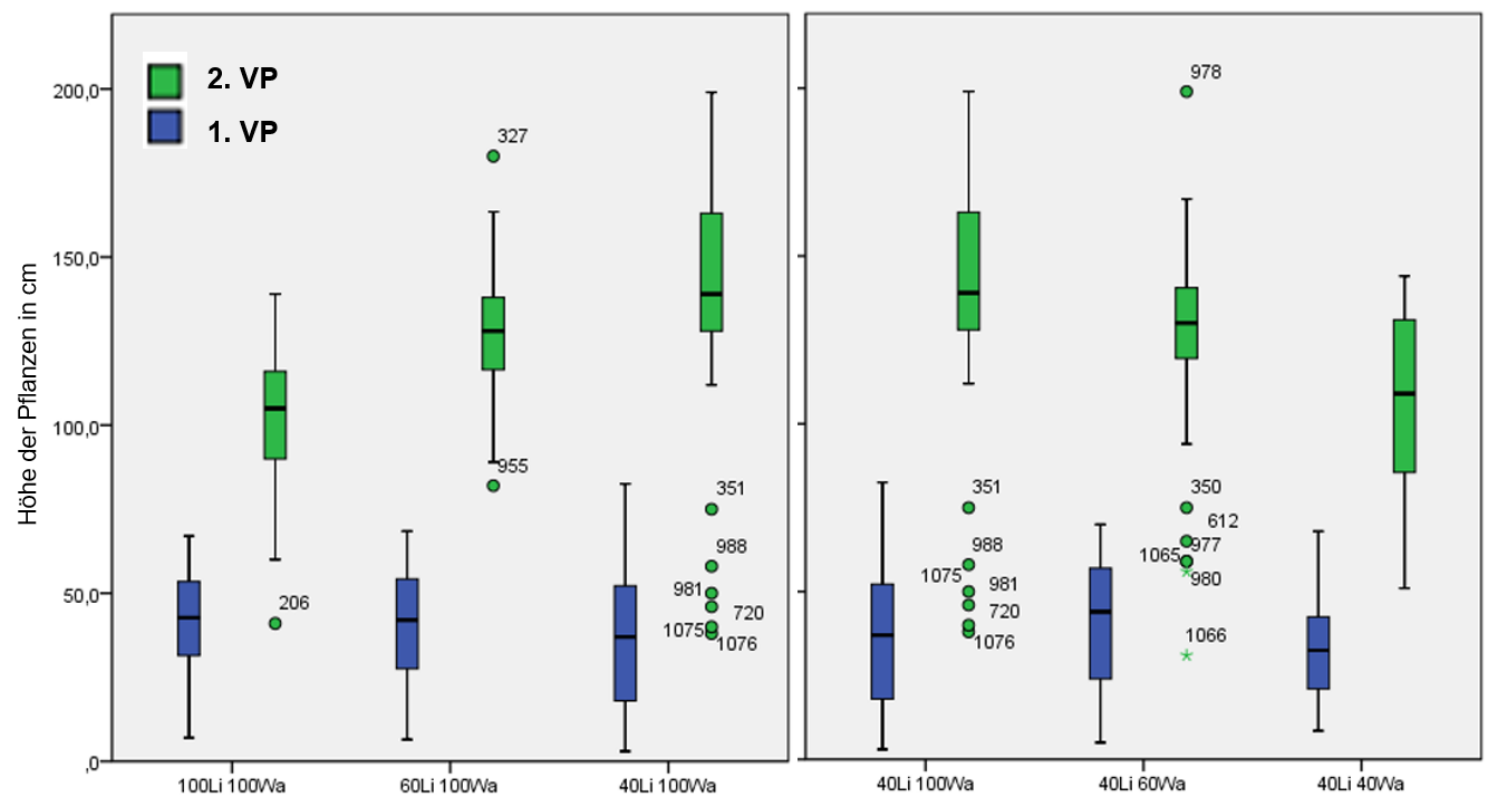

Abb. 25: Vergleich des Längenwachstums aller Paulownia-Pflanzen zwischen der ersten und zweiten Vegetationsperiode (VP). Linke Grafik beschreibt unterschiedliche Lichtstufen (100Li, 60Li, 40Li) bei gleichbleibender $100 \%$ Bewässerung. Rechte Grafik beschreibt die Lichtstufe $40 \%$ bei abnehmender Bewässerung (100Wa, 60Wa, 40Wa).

Erst am Ende der zweiten Vegetationsperiode sind signifikante Unterschiede zwischen den Behandlungsarten Licht und Wasser erkennbar. Mit abnehmendem Licht und gleichbleibend hoher Wasserverfügbarkeit, steigt das Höhenwachstum in der zweiten Vegetationsperiode, wohin gegen das Höhenwachstum mit Verminderung der Wasserverfügbarkeit abnimmt. Jedoch ist in beiden Vegetationsperioden innerhalb der Behandlungsstufen eine große Spannweite im Längenwachstum der Pflanzen vorhanden.

Diese Spannweite ist bspw. deutlich bei der $40 \%$ Lichtstufe zu $100 \%$ Wasser sichtbar. Ein Teil der Pflanzen ist ganz normal in der zweiten Vegetationsperiode wieder ausgetrieben, aber im Längenwachstum stark zurückgeblieben, wodurch sie aus dem eigentlichen Boxplot herausfallen und graphisch als Ausreißer dargestellt werden. 
Es bildet sich folglich eine Art zweischichtiger Bestand heraus von stark in die Höhe strebenden Pflanzenindividuen auf der einen Seite und zurückgebliebenen Pflanzen auf der anderen Seite, die zwar überleben aber nur noch vermindert in die Länge wachsen. Es zeigt sich beispielsweise, dass bei geringer werdendem Lichtangebot und bei gleichbleibend hoher Wasserversorgung die P.-Pflanzen in ein verstärktes Längenwachstum, welches bei $40 \%$ Licht und $100 \%$ Wasserversorgung am höchsten ausfällt, investieren. Im Gegensatz dazu ist es bei $100 \%$ Licht und $40 \%$ Wasser am geringsten (Abb. 26).

Bei weiterhin geringer Wasserversorgung (40 \%) und weiterer Reduzierung des Lichtes auf $60 \%$ und $40 \%$ nimmt das Längenwachstum wieder zu. Wobei sich die Mittelwerte bei $60 \%$ und $40 \%$ Licht und $40 \%$ Wasser, sowohl in der ersten wie auch in der zweiten Vegetationsperiode, nicht signifikant voneinander unterscheiden.

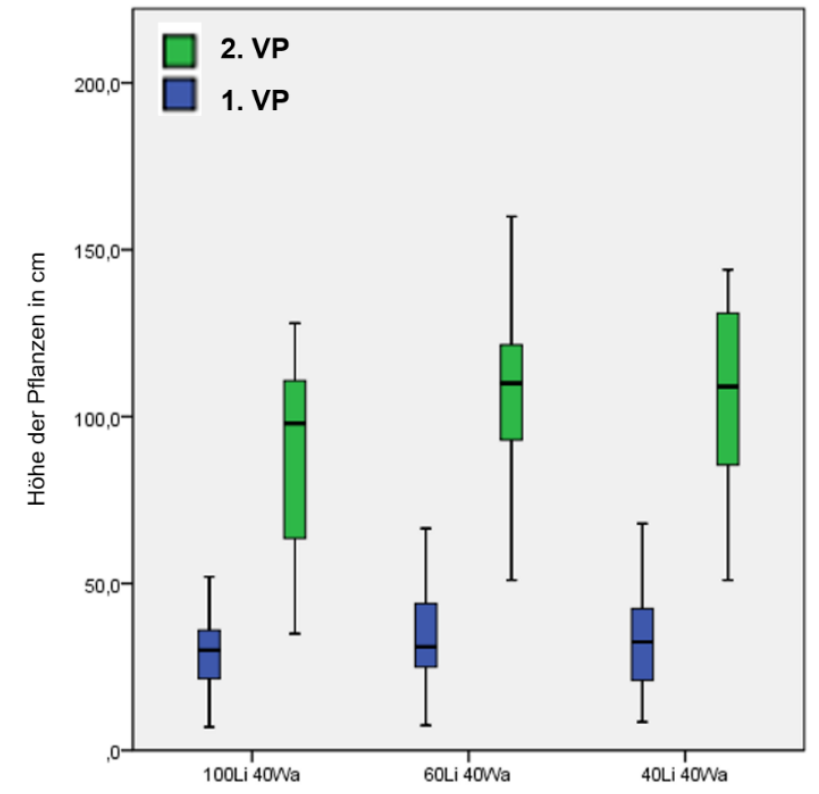

Abb. 26: Vergleich des Längenwachstums aller Paulownia-Pflanzen zwischen der ersten und zweiten Vegetations-periode (VP), bei abnehmenden Lichtstufen (100Li, 60Li, 40Li) und gleichbleibender 40 $\%$ Bewässerung.

\subsection{Durchmesserwachstum}

Im Vergleich von der ersten zur zweiten Vegetationsperiode erhöht sich der mittlere Pflanzendurchmesser, wobei es sich hierbei um einen kompletten Neuaustrieb handelt. Dieser Durchmesserzuwachs erfolgt aber nicht im gleichen Verhältnis wie er beim Längenwachstum zu beobachten ist (Abb. 27).

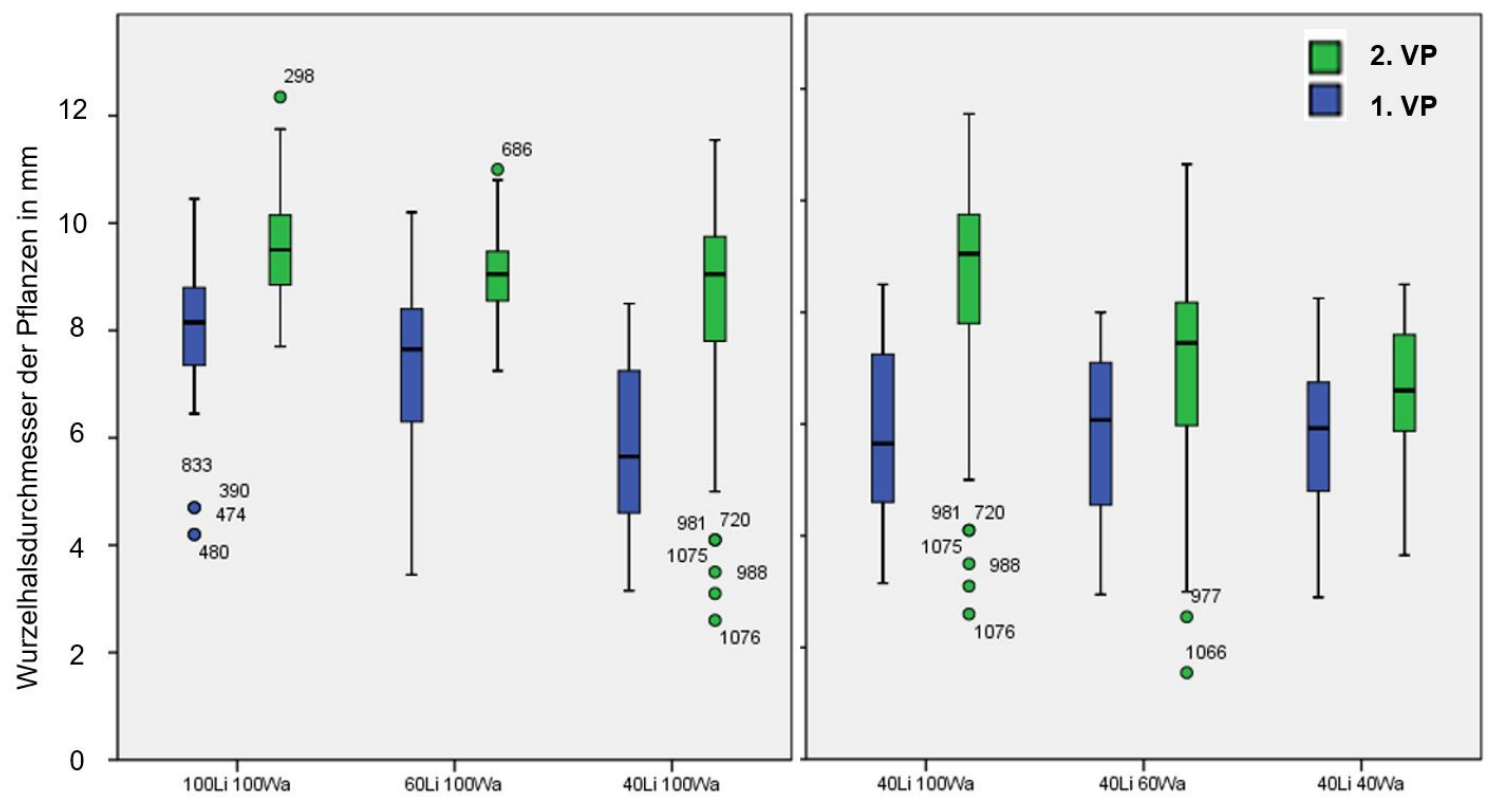

Abb. 27: Vergleich des Wurzelhalsdurchmessers zwischen erster und zweiter Vegetationsperiode (VP). Linke Grafik beschreibt unterschiedliche Lichtstufen $(100 \mathrm{Li}, 60 \mathrm{Li}, 40 \mathrm{Li})$ bei gleichbleibender $100 \%$ Bewässerung. Rechte Grafik beschreibt die $40 \%$ Lichtstufe bei abnehmender Bewässerung (100Wa, 60Wa, 40Wa). 
Bei der $40 \%$ Lichtstufe zeigen die Pflanzen eine größere Spannweite in ihrer Durchmesserentwicklung als bei der $100 \%$ Lichtstufe (Abb. 28). Hierbei handelt es sich um die gleiche Beobachtung wie sie schon im Zusammenhang mit dem Längenwachstum thematisiert wurde, bezüglich der Ausbildung schwacher und starker Pflanzenindividuen. Dieser Unterschied zwischen den Lichtstufen lässt sich auch in der zweiten Vegetationsperiode beobachten.

Bei der $100 \%$ Lichtstufe geht mit einer Verringerung der Wasserzufuhr auch ein verminderter Durchmesserzuwachs einher, was in beiden Vegetations-perioden gleich ist.

Die Pflanzen können folglich das volle

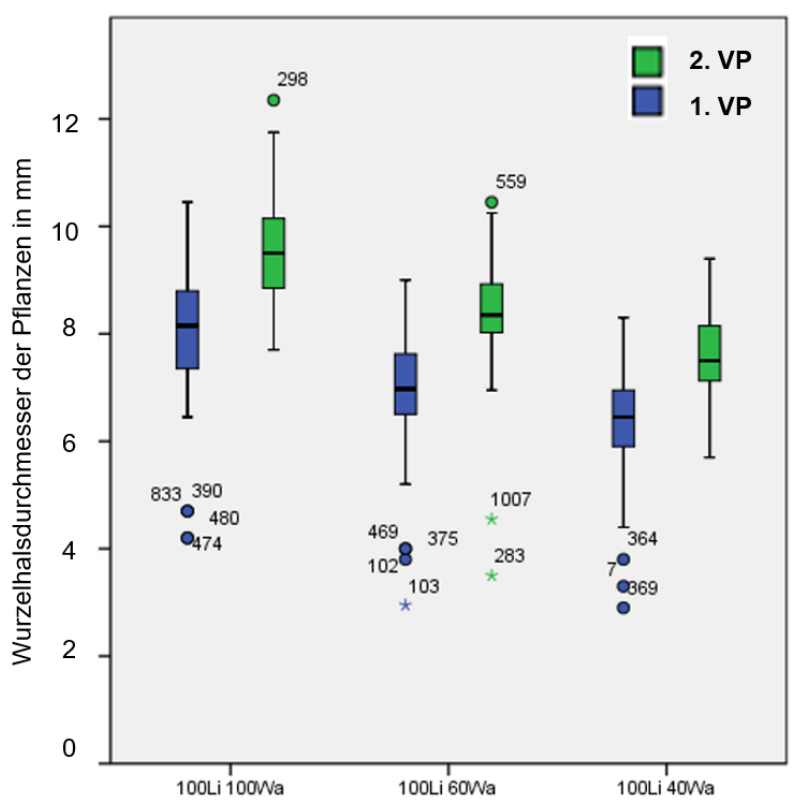

Abb. 28: Vergleich des Wurzelhalsdurchmessers zwischen erster und zweiter Vegetationsperiode (VP) bei gleichbleibender $100 \%$ Lichtstufe und abnehmender Bewässerung (100Wa, 60Wa, 40Wa). Licht zum Wachstum ausnutzen, und die Limitation im Durchmesserwachstum wird durch die Wasserzufuhr bestimmt. Im Gegensatz dazu ist bei der $40 \%$ Lichtstufe dieser Effekt erst in der zweiten Vegetationsperiode zu beobachten. Die verabreichte Wassermenge wirkt somit bei sehr geringer Lichtzufuhr in der ersten Vegetationsperiode noch nicht limitierend.

Um eine Aussage über die Stabilität von Bäumen zu treffen, wird oft das H/D-Verhältnis herangezogen. Dabei wird die Höhe im Verhältnis zum Durchmesser gesetzt, und es zeigt sich in der zweiten Vegetationsperiode eine deutliche Zunahme des H/DVerhältnisses und eine große Streuung der Werte (Abb. 29).

Die Mehrzahl der Pflanzen zeigt ein in Proportion zueinander stehendes Längen- und Durchmesserwachstum. Die Streuung erklärt sich durch besonders lange und dünne bzw. kurze und dicke Pflanzenstängel. Sie sind eine Folge des Neuaustriebes und der unterschiedlichen Entwicklung der Pflanzen.

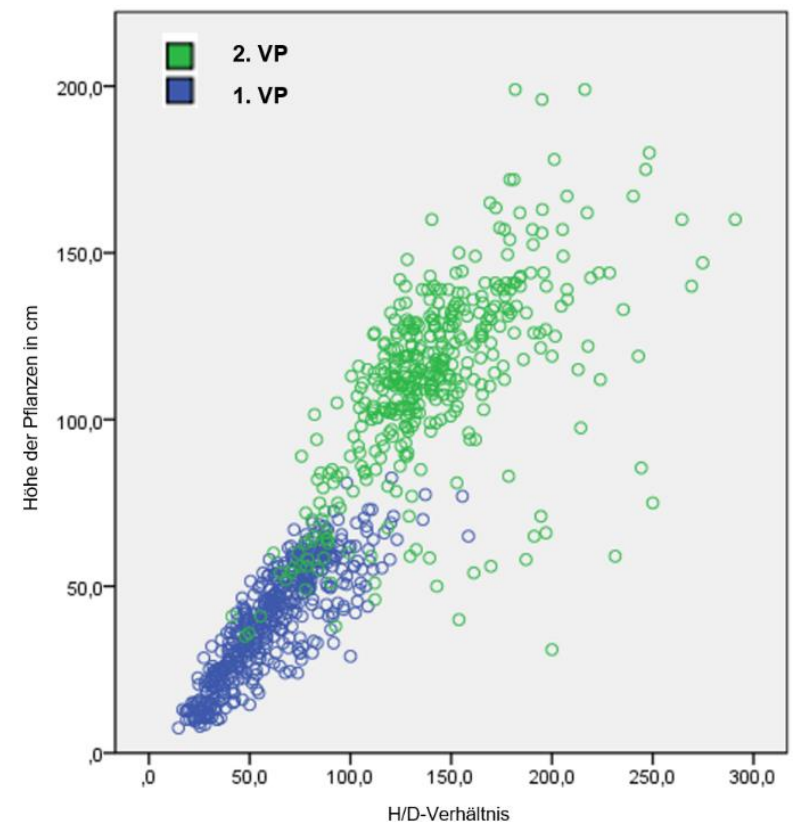

Abb. 29: Verhältnis von Höhe zu Durchmesser in erster (blau) und zweiter (grün) Vegetationsperiode (VP). 


\subsection{Trockenmasse}

Am Ende jeder Vegetationsperiode wurde die gesamte oberirdische Biomasse, also der verbleibende Pflanzenstängel ohne Blätter abgeerntet, getrocknet und gewogen. Im Folgenden wird daher nur noch von Trockenmasse bzw. Biomasse gesprochen.

Die vorab beschriebenen Beobachtungen beim Längen- und Durchmesserwachstum finden sich auch in ähnlicher Form beim Vergleich der oberirdischen Biomasse (Stängel ohne Blattmasse) wieder (Abb. 30).

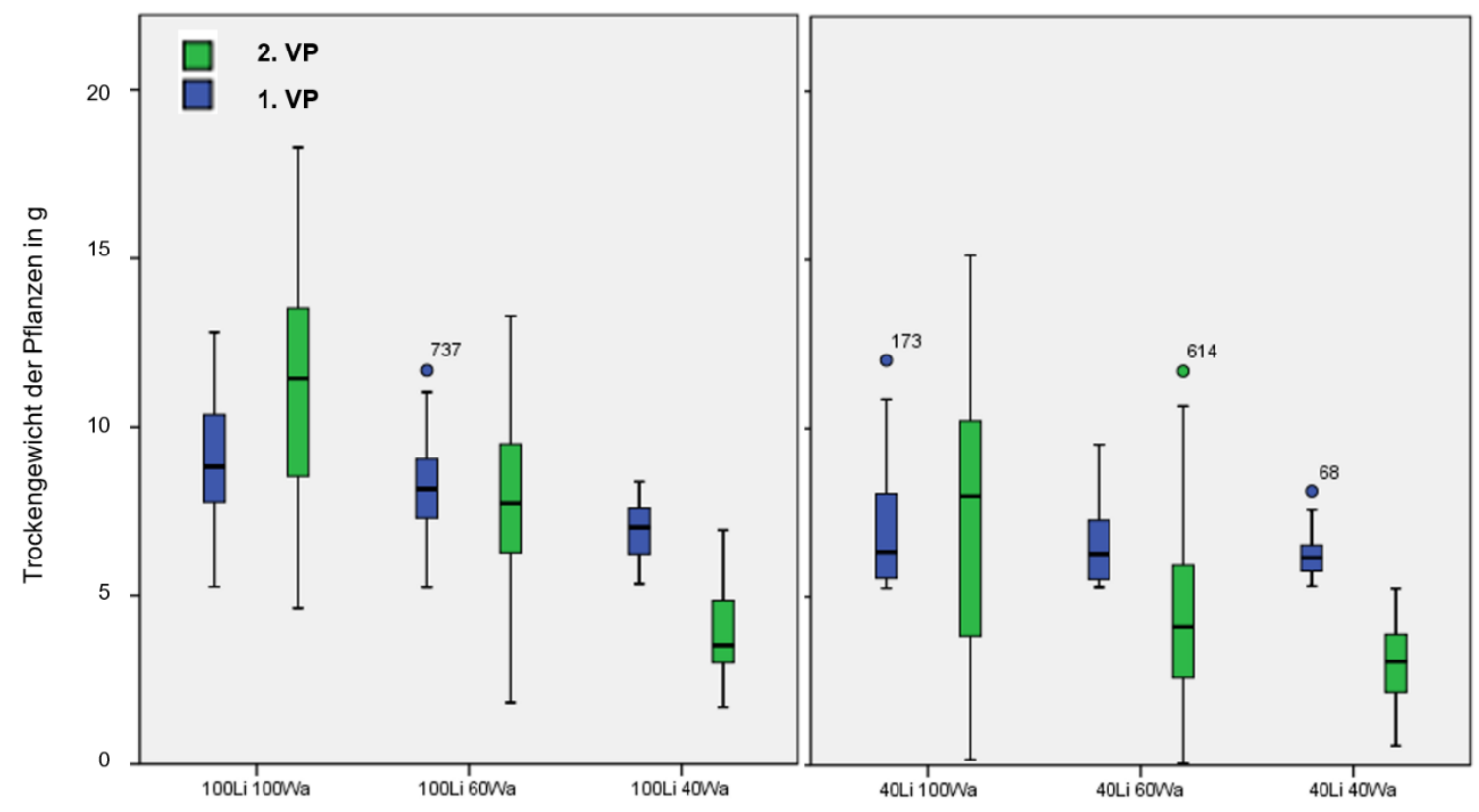

Abb. 30: Vergleich des Trockengewichts (in Gramm) aller Paulownia-Pflanzen zwischen der ersten und zweiten Vegetationsperiode (VP). Linke Grafik mit $100 \%$ Licht, rechte Grafik mit $40 \%$ Licht bei jeweils unterschiedlicher Bewässerung (100Wa, 60Wa, 40Wa).

Generell ist die Trockenmasse der Pflanzen umso größer, je mehr Wasser zur Verfügung steht, wobei die Lichtstufe keinen nachweislichen Einfluss nimmt.

Bei den Behandlungsstufen von 100 und $60 \%$ Licht zu jeweils 100 und $60 \%$ Wasser gibt es bei der Ausbildung der Stängelbiomasse keinen signifikanten Unterschied zwischen der ersten und zweiten Vegetationsperiode.

Die Pflanzen haben folglich in der zweiten Vegetationsperiode weniger Masse aufgebaut als es zu erwarten war und dass, trotz eines stärker ausgeprägten Längen- und Dickenwachstums.

Wenn der Pflanzenstängel im Durchmesser zunimmt, so bildet sich in seiner Mitte ein Hohlraum. Der Stängel ist folglich kernlos und erinnert im Querschnitt an eine dickwandige Röhre. Dieser Hohlraum ist auch noch bei der Ernte des ausgewachsenen Baumes deutlich erkennbar.

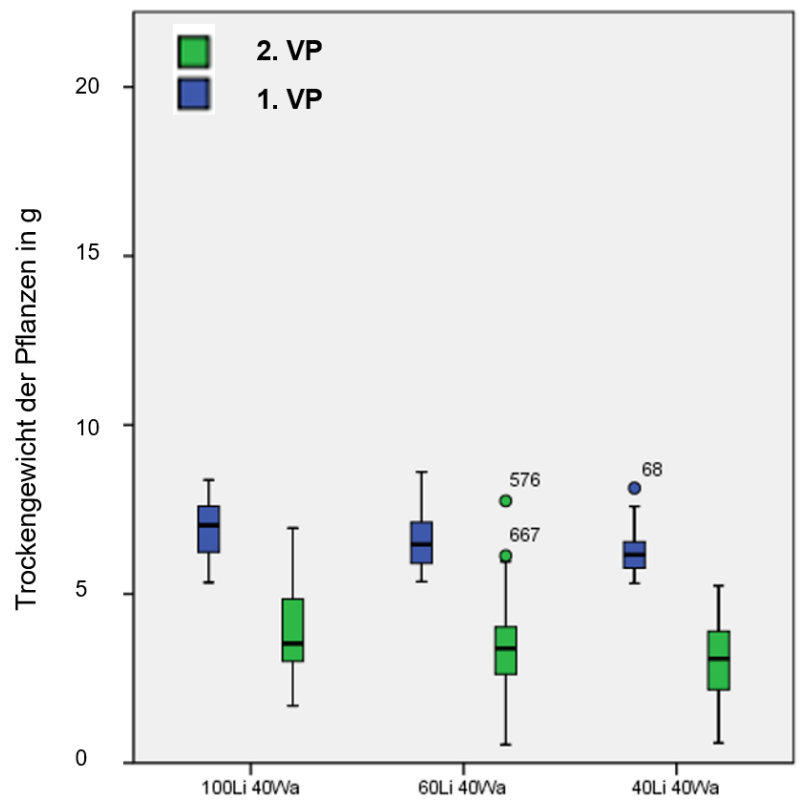

Abb. 31: Vergleich des Trockengewichts aller Paulownia-Pflanzen zwischen der ersten und zweiten Vegetationsperiode (VP). Bei unterschiedlichen Lichtstufen (100Li, 60Li, 40Li) und $40 \%$ Bewässerung. 
Erst bei der $40 \%$ Bewässerungsstufe (Abb. 31) ist der Biomasseaufbau der zweiten Vegetationsperiode signifikant kleiner als in der ersten, wobei die jeweilige Lichtstufe vernachlässigbar ist.

Die Pflanzen waren folglich in der $40 \%$ Bewässerungsstufe nicht in der Lage, in der ersten Vegetationsperiode genügend Reservestoffe aufzubauen, um in der zweiten Vegetationsperiode gleich viel oder mehr Biomasse zu bilden, obwohl sie ein signifikant stärkeres Längen- als auch Dickenwachstum zeigen.

Um das Gesamtbild zu ergänzen, bietet sich eine Betrachtung der Höhe im Verhältnis zum Gewicht an (Abb. 32).

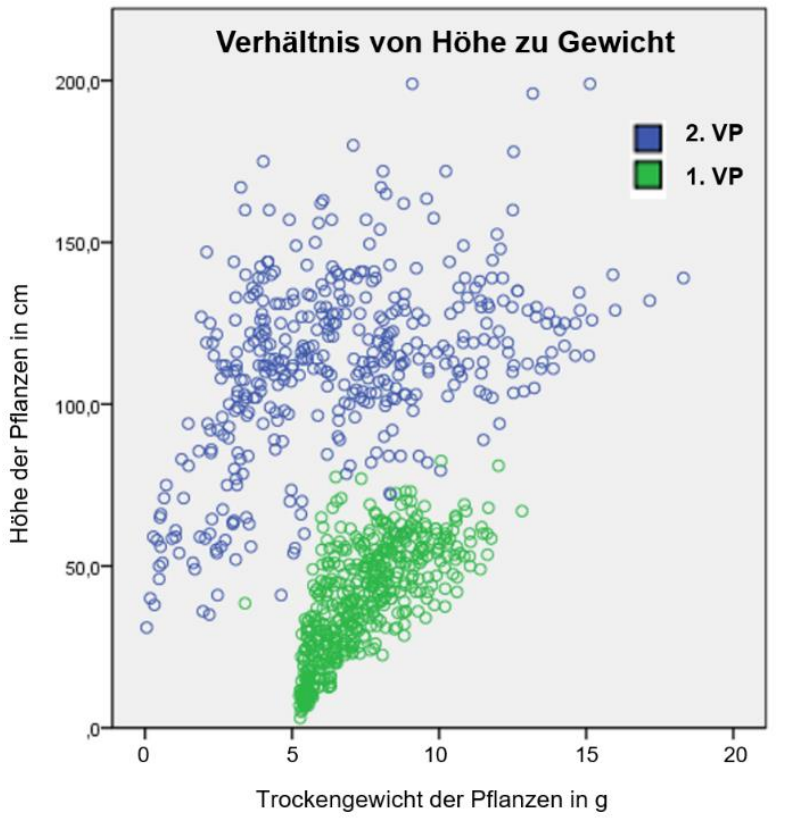

Abb. 32: Verhältnis von Höhe zu Gewicht aller Paulownia-Pflanzen im Vergleich von erster (grün) zu zweiter (blau) Vegetationsperiode (VP).

Hierbei zeigt sich eine deutlich größere Spannweite in der zweiten Vegetationsperiode (blau in der Grafik). Nahezu die Hälfte aller Pflanzen ist in der zweiten Vegetationsperiode, im Vergleich zur ersten, leichter aber trotzdem fast doppelt so lang. Die Einzelpflanze erzielt unter Ausbildung von weniger bzw. gleich viel Stängelbiomasse ein größeres Längenwachstum und ein proportional dazu nötiges Dickenwachstum.

\subsubsection{Jugendwachstum bei einem Vergleich der Arten}

Auf Grundlage der verschiedenen Behandlungsarten und -stufen zeigen die drei P.Arten (P.elongata, P.fortunei, P.tomentosa) unterschiedliche Reaktionen in ihrer Entwicklung, die nur selten signifikant voneinander verschieden sind. Die Wachstumsreaktionen sind der Vorab-Betrachtung auf Gattungsebene ähnlich.

Im Folgenden werden die Ergebnisse auf Artebene dargestellt, wobei zeitlich weiterhin zwischen erster und zweiter Vegetationsperiode unterschieden und nur die Baumart hervorgehoben wird, die den zu beschreibenden Effekt am deutlichsten ausprägt.

Der dargestellte Stichprobenumfang verringert sich somit pro Boxplot auf maximal 20 gleichbehandelte Pflanzenindividuen, abzüglich der jeweiligen Ausfälle (Tab. 7). 


\subsection{Längenwachstum}

Wie bereits auf Gattungsebene erkennbar ist, zeigt sich auch auf Artebene kein signifikanter Unterschied im Längenwachstum der P.-Pflanzen zum Ende der ersten

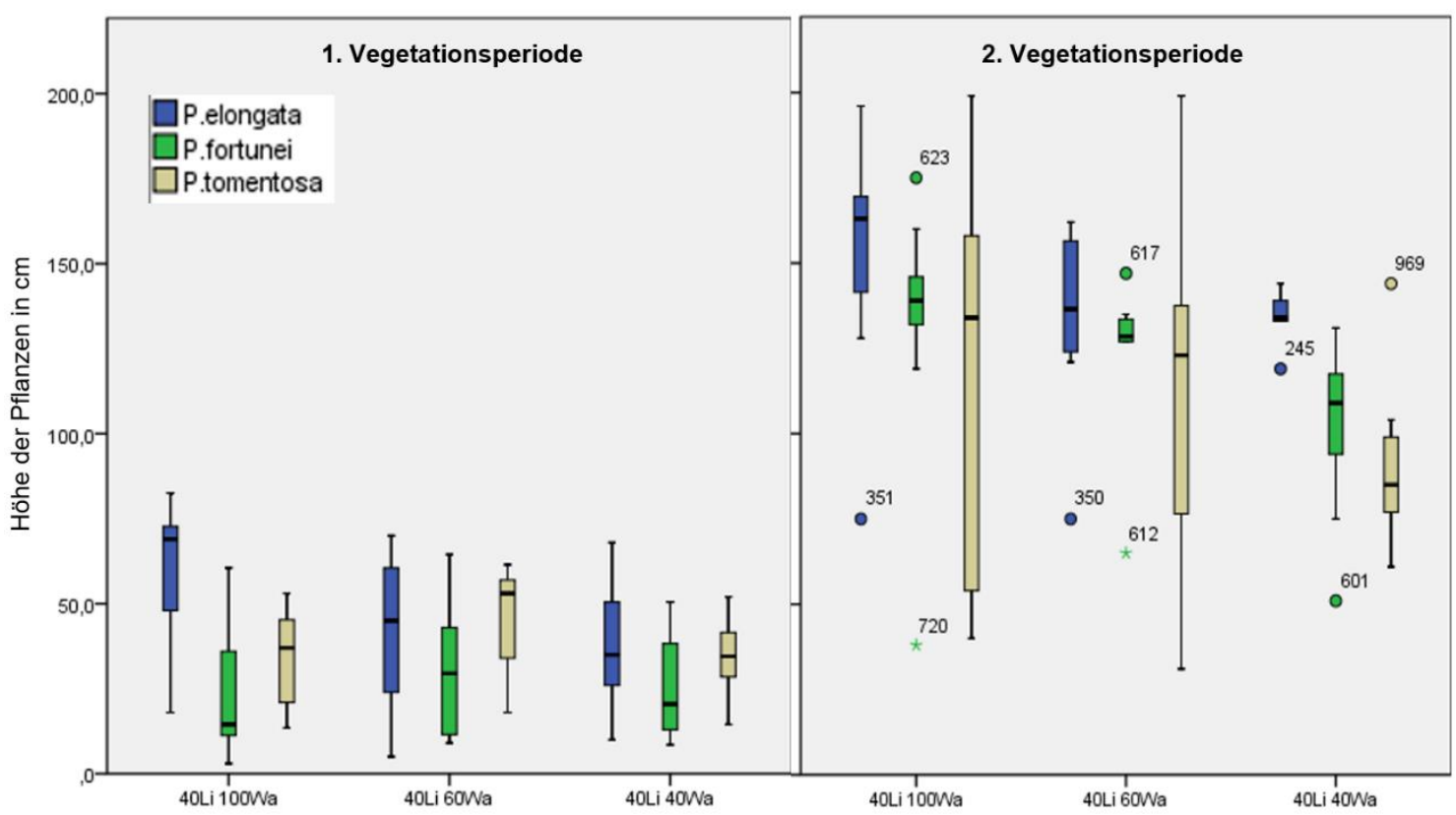

Abb. 33: Vergleich des Längenwachstums der Paulownia-Arten bei der $40 \%$ Lichtstufe und unterschiedlicher Wasserversorgung (100Wa, 60Wa, 40Wa). Linke Grafik beschreibt erste und rechte Grafik die zweite Vegetationsperiode.

Vegetationsperiode (Abb. 33). Erst in der zweiten Vegetationsperiode gibt es eine klare Differenzierung zwischen den Behandlungsstufen, wobei sich die Arten zumeist sehr ähnlich sind und es bei gleichbleibend hoher Wasserversorgung (100\%) und mit Abnahme des Lichtes zu einer Zunahme des Längenwachstums kommt.

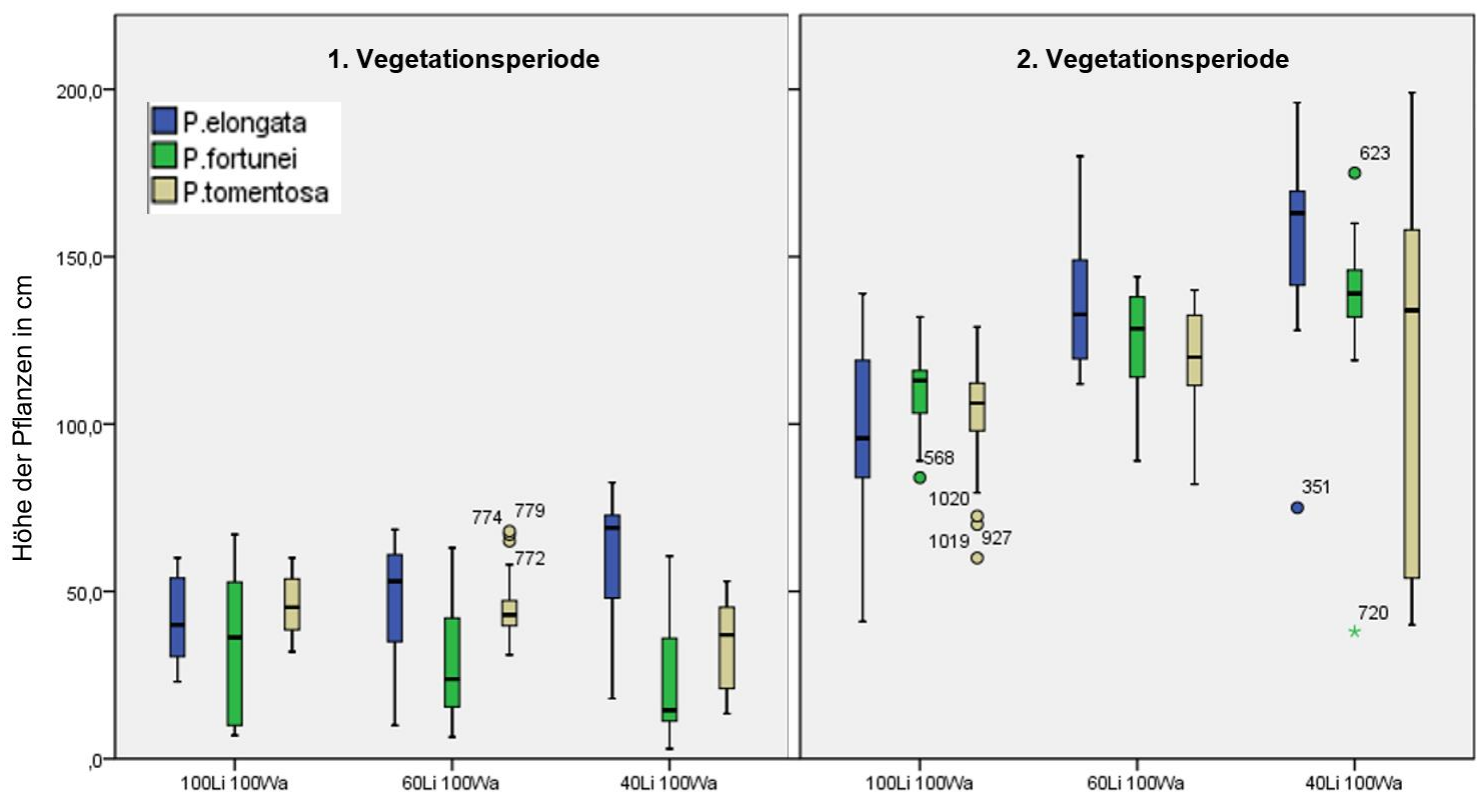

Abb. 34: Vergleich des Längenwachstums der Paulownia-Arten bei $100 \%$ Bewässerung aber unterschiedlichen Lichtstufen (100Li, 60Li, 40Li). Linke Grafik beschreibt erste und rechte Grafik die zweite Vegetationsperiode. 
Bei der $40 \%$ Lichtstufe ist die Ausdifferenzierung zwischen den drei Bewässerungsstufen, ab der zweiten Vegetationsperiode, am stärksten ausgeprägt. Wenn folglich bei schwachem Licht (40\%) die Wasserzufuhr weiter reduziert wird, verringert sich auch das Längenwachstum.

Im Gegensatz dazu zeigen alle Pflanzen bei einer Verringerung der Lichtzufuhr (100\%, $60 \%, 40 \%$, Abb. 34) eine Zunahme des Längenwachstums, welches bei $40 \%$ Licht und $100 \%$ Wasser am stärksten ausfällt.

P.tomentosa zeigt im Vergleich zu den anderen beiden Arten die größte Spannweite beim Längenwachstum in der zweiten Vegetationsperiode. Dies ist darauf zurückzuführen, dass die Baumart die vergleichsweise geringste Ausfallrate hat und somit auch Pflanzenindividuen miterfasst werden, die nur wenig Wachstum zeigten aber dennoch überlebten.

Die größte Gesamtlänge erreicht bei 40 \% Belichtung und $100 \%$ Bewässerung jedoch P.elongata mit $54 \mathrm{~cm}$ in der ersten und $156,5 \mathrm{~cm}$ in der zweiten Vegetationsperiode.

Ganz allgemein betrachtet, sind Reaktionen auf die verschiedenen Behandlungsstufen erkennbar, aber es gibt keine signifikanten Unterschiede zwischen den drei P.-Arten. Signifikanzen treten vereinzelt nur zwischen den Behandlungsstufen auf.

\subsection{Durchmesserwachstum}

In der zweiten Vegetationsperiode erhöht sich das Durchmesserwachstum im Verhältnis zur ersten Vegetationsperiode. Dieses zusätzliche Wachstum fällt aber nicht so stark aus wie bspw. beim Längenwachstum.

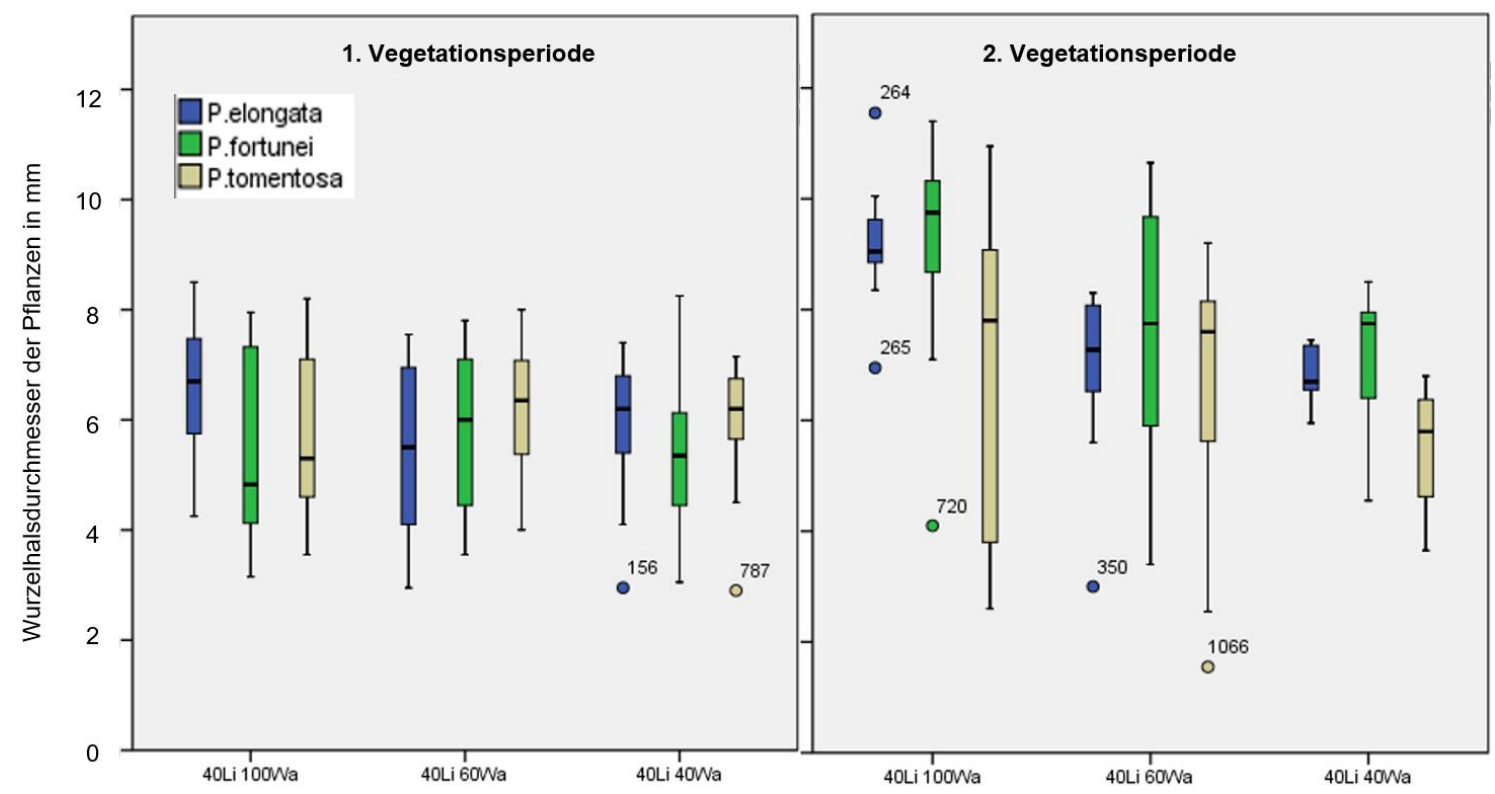

Abb. 35: Vergleich des Wurzelhalsdurchmessers der P.-Arten zwischen erster (linke Grafik) und zweiter (rechte Grafik) Vegetationsperiode. Beide Grafiken zeigen die $40 \%$ Lichtstufe bei unterschiedlicher Bewässerung (100Wa, 60Wa, 40Wa).

Eine klare Ausdifferenzierung zwischen den Behandlungsstufen zeichnet sich auch hier erst in der zweiten Vegetationsperiode ab.

In der ersten Vegetationsperiode gibt es bspw. bei $40 \%$ Licht (Abb. 35) keine signifikanten Unterschiede zwischen den verschiedenen Bewässerungsstufen. 
Bei konstant hoher Wasserzufuhr (100\%) ist mit Abnahme des Lichtes ein Rückgang des Durchmessers in der ersten Vegetationsperiode zu beobachten, der aber sowohl zwischen den Stufen als auch zwischen den Arten nicht signifikant und in der zweiten Vegetationsperiode weniger deutlich erkennbar ist.

Mit Verringerung der Wasserzufuhr nimmt auch der Durchmesser von Stufe zu Stufe ab, wie in der zweiten Vegetationsperiode klar erkennbar ist.

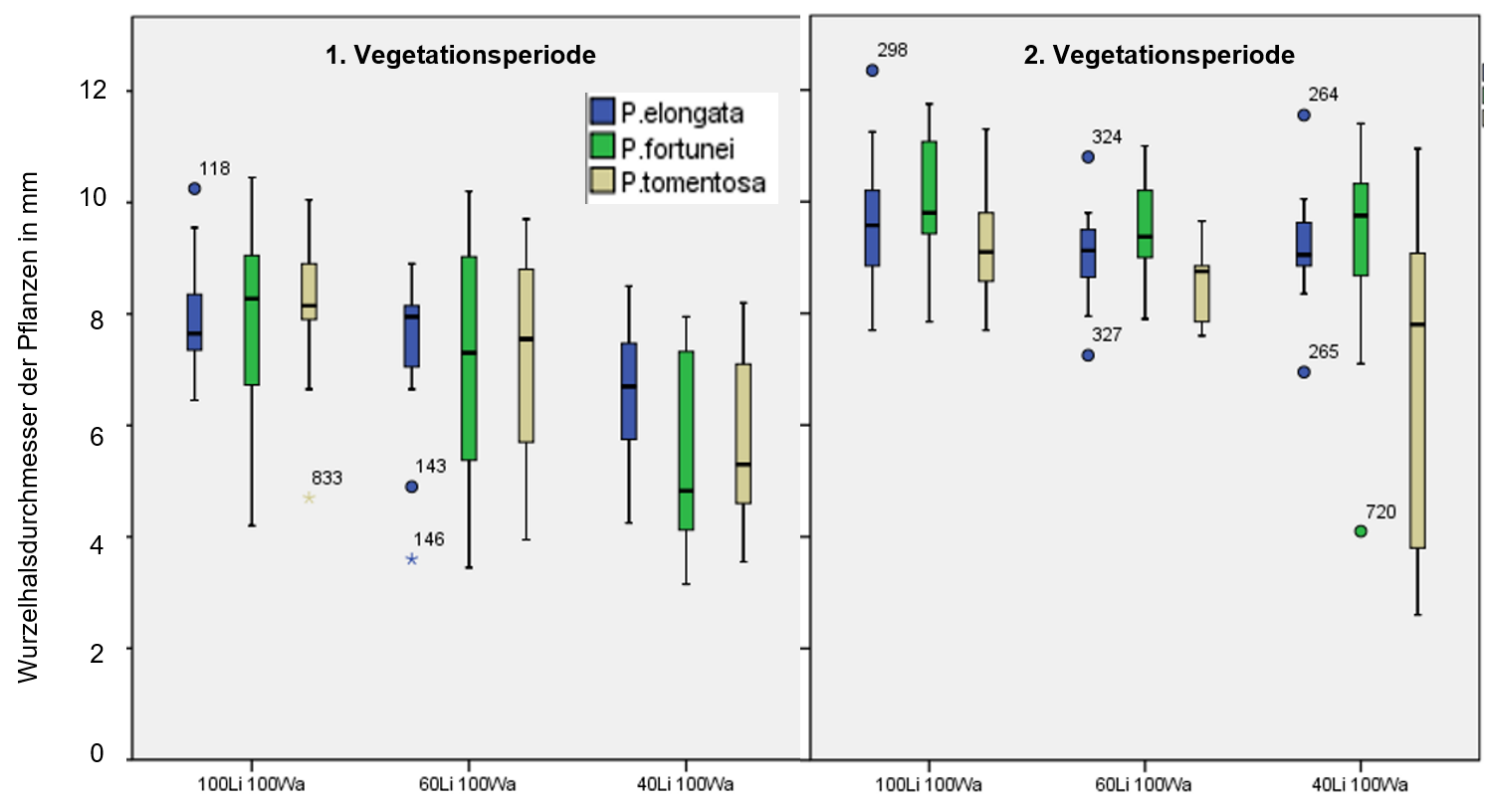

Abb. 36: Vergleich des Wurzelhalsdurchmessers der P.-Arten zwischen erster (linke Grafik) und zweiter (rechte Grafik) Vegetationsperiode. Beide Grafiken zeigen die $100 \%$ Bewässerungsstufe bei unterschiedlicher Lichtzufuhr (100Li, 60Li, 40Li).

Anders als es beim Längenwachstum zu beobachten ist, zeichnet sich mit Abnahme des Lichtes keine Zunahme des Durchmesserwachstums ab (Abb. 36). Folglich kommt es hier zu einer Verschiebung des Verhältnisses von der Höhe zum Durchmesser (H/DVerhältnis). Die Pflanzenstängel werden zwar länger mit Abnahme des Lichtes (Längenzuwachs), bleiben aber im Durchmesser unverändert. Ihre Stabilität nimmt somit ab.

Die beobachteten Reaktionen der Baumarten gleichen denen beim Längenwachstum. P.tomentosa bildet in dem Extrembereich von $40 \%$ Licht auch weiterhin die größte Spannweite ab. P.elongata und P.fortunei ähneln sich in ihren Reaktionen bezüglich der Behandlungsstufen.

\subsection{Trockenmasse}

Auch bei der Stängelbiomasse lässt sich in der ersten Vegetationsperiode keine Reaktion der Pflanzen auf die verschiedenen Behandlungsstufen beobachten. Die Spannweiten der P.-Arten sind im Vergleich zum Längen- und Durchmesserwachstum bei der Biomasse am geringsten ausgeprägt, wobei sie bei eingeschränkter Wasserzufuhr (40\%) am kleinsten ausfallen. Eine Ausdifferenzierung zwischen den P.Arten ist nicht erkennbar. 
Im Gegensatz zum Längenwachstum verringert sich die Biomasse bei gleichbleibender hoher Bewässerung (100 \%) mit Abnahme der Lichtzufuhr in der zweiten Vegetationsperiode, wobei dieser in den Grafiken erkennbare Unterschied nicht signifikant ist (Abb. 37).

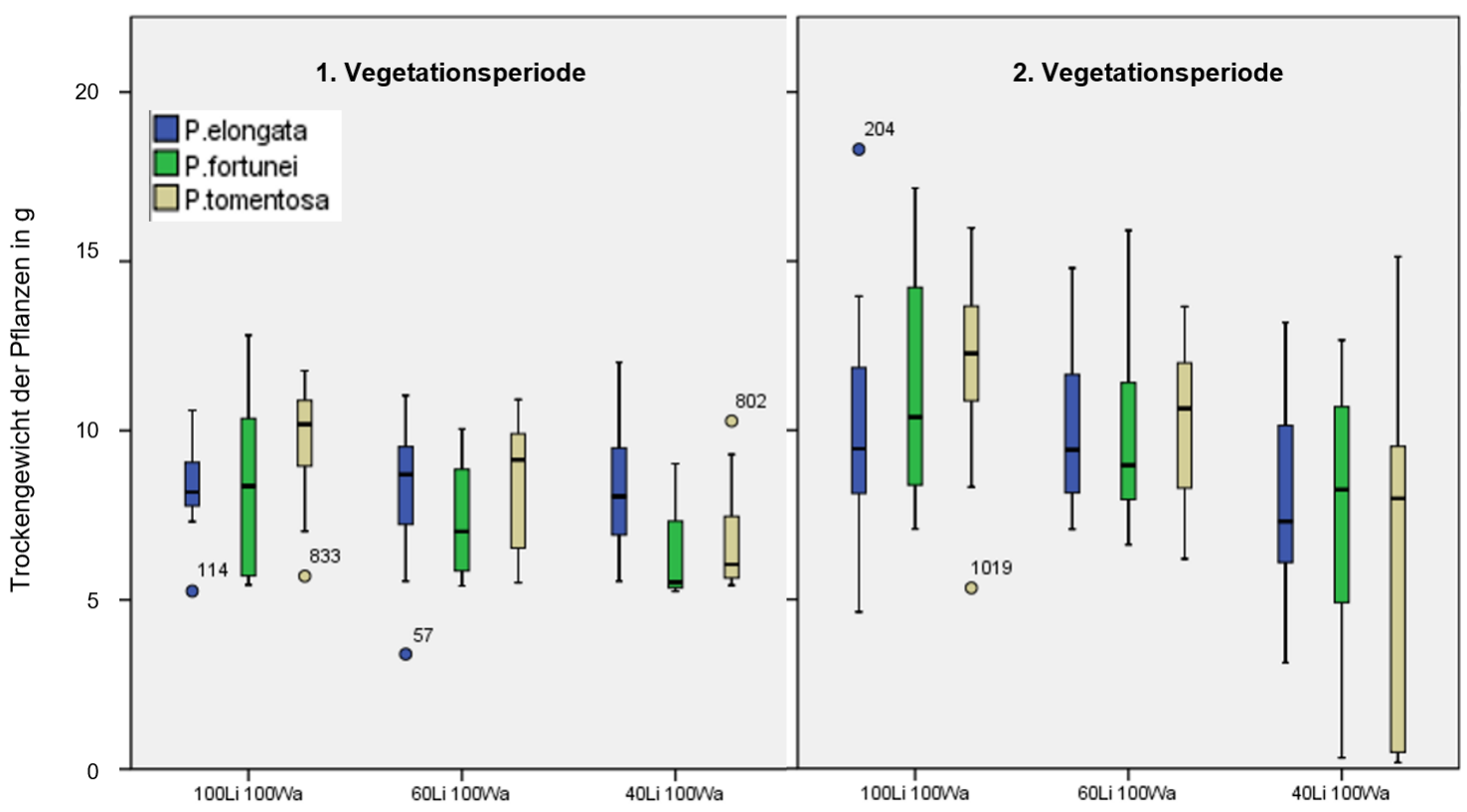

Abb. 37: Vergleich des Trockengewichts der Paulownia-Arten zwischen der ersten (linke Grafik) und zweiten (rechte Grafik) Vegetationsperiode. Jeweils mit $100 \%$ Bewässerung bei unterschiedlicher Belichtung (100Li, 60Li, 40Li).

Erst bei einer Kombination der Behandlungsstufen von gleichbleibend hoher Lichtzufuhr (100\%) und unter Abnahme der Bewässerung von $100 \%$ auf $60 \%$ und $40 \%$ verringert sich die Biomasse der Pflanzen in der zweiten Vegetationsperiode signifikant (Abb. 38).

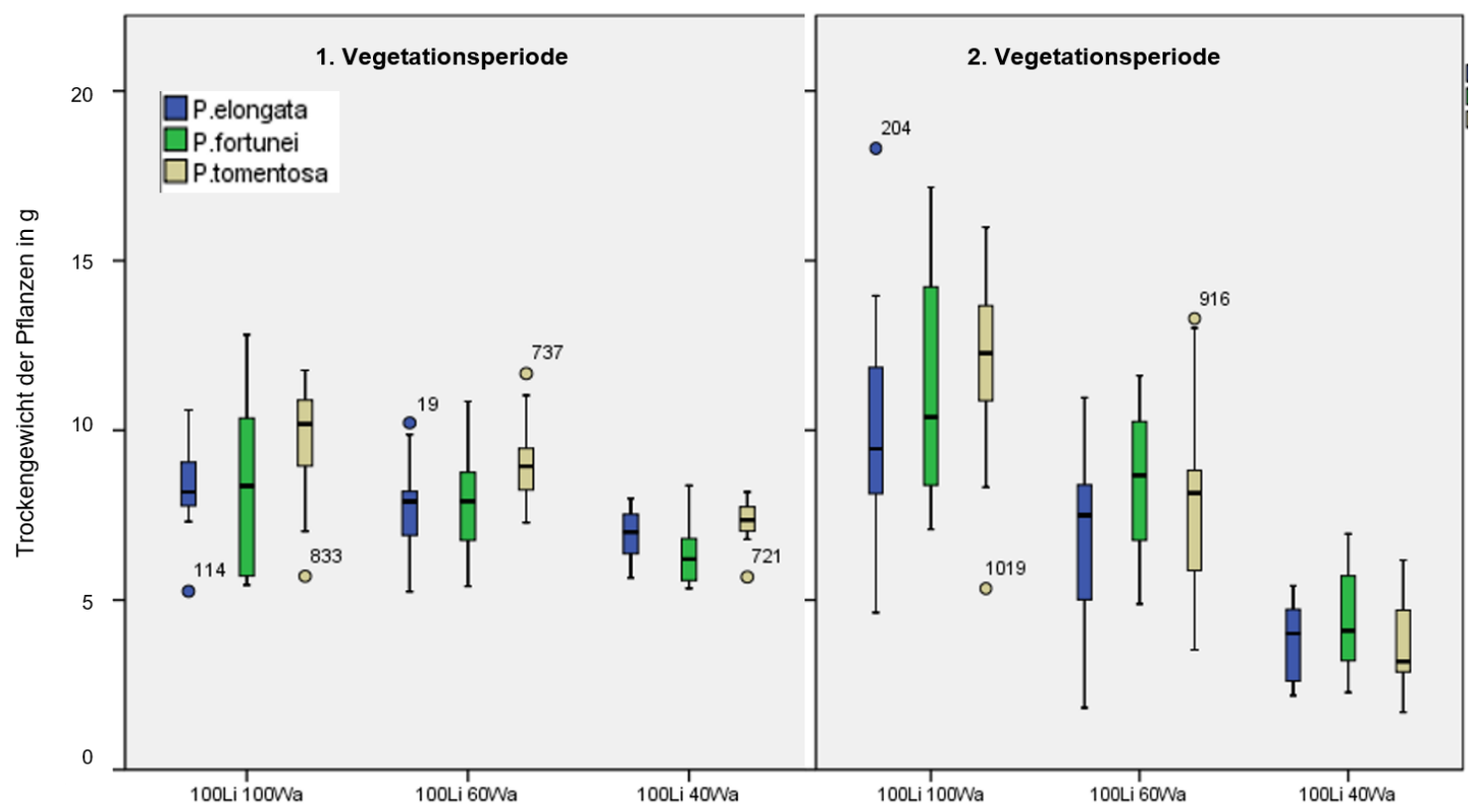

Abb. 38: Vergleich des Trockengewichts der Paulownia-Arten zwischen der ersten (linke Grafik) und zweiten (rechte Grafik) Vegetationsperiode. Jeweils mit $100 \%$ Licht bei unterschiedlicher Bewässerung (100Wa, 60Wa, 40Wa). 
Bei unterschiedlicher Lichtzufuhr aber nur sehr geringer Bewässerung (40\%) bilden die Pflanzen in der zweiten Vegetationsperiode signifikant weniger Stängelbiomasse als in der ersten (Abb. 39).

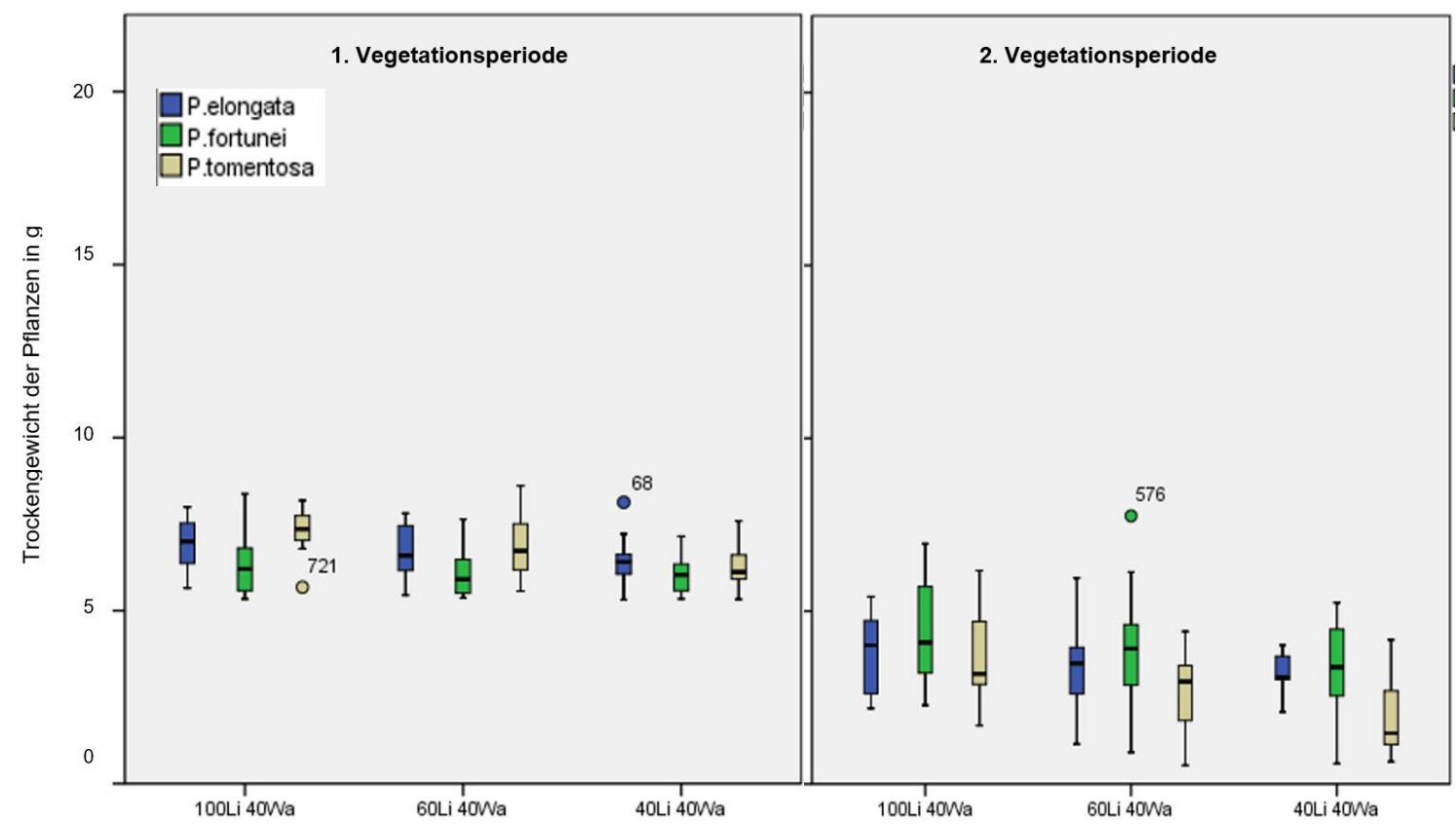

Abb. 39: Vergleich des Trockengewichts der Paulownia-Arten zwischen der ersten (linke Grafik) und zweiten (rechte Grafik) Vegetationsperiode. Jeweils mit $\mathbf{4 0} \%$ Bewässerung bei unterschiedlicher Lichtzufuhr (100Li, 60Li, 40Li).

Die Reaktionen der Pflanzen in der zweiten Vegetationsperiode müssen also eine Folge der Behandlungsstufen der ersten Vegetationsperiode sein. Die Pflanzen versuchen in der zweiten Vegetationsperiode zu überleben, sind aber nicht mehr in der Lage, die gleiche Trockenmasse wie in der Vegetationsperiode zuvor zu bilden.

\subsection{Blattfläche, Blattanzahl und Triebanzahl}

Zum Ende der zweiten Vegetationsperiode wurde noch vor dem natürlichen Blattfall eine Vollaufnahme an den lebenden Pflanzen durchgeführt. In einem Zeitraum von zwei Wochen wurden die Blätter aller Pflanzen vermessen. Aufgeschlüsselt nach Behandlungsart und -stufen lassen sich dabei die folgenden Rückschlüsse ziehen.

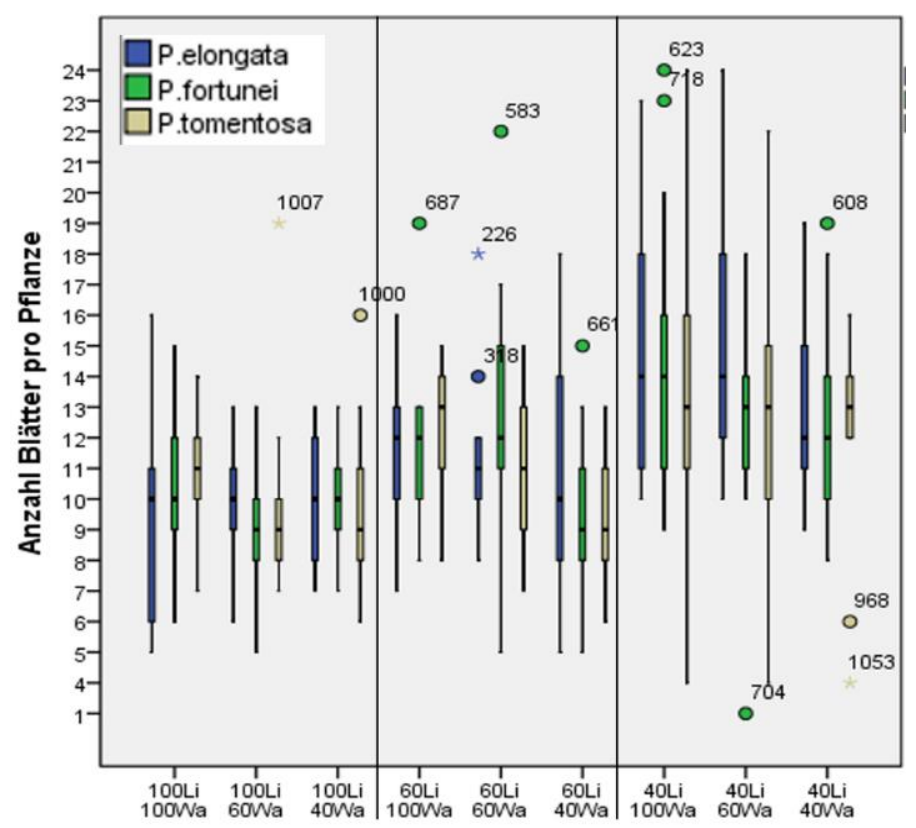

Abb. 40: Anzahl Blätter pro Pflanze unterteilt nach Behandlungsstufen (Licht und Wasser) und aufgeschlüsselt nach Paulownia-Arten. 
Bei der Blattanzahl gibt es signifikante Unterschiede zwischen den Behandlungsstufen aber nicht zwischen den P.-Arten (Abb. 40). Die Spannweiten sind zumeist sehr groß, was für eine hohe Heterogenität der Pflanzenindividuen spricht.

Bei den Lichtstufen $100 \%$ und $60 \%$ zeigen sich keine signifikanten Unterschiede in der Blattanzahl ganz gleich welche Bewässerungsstufen betrachtet werden.

Erst bei einer sehr geringen Lichtzufuhr von $40 \%$ bilden die Pflanzen mehr Blätter

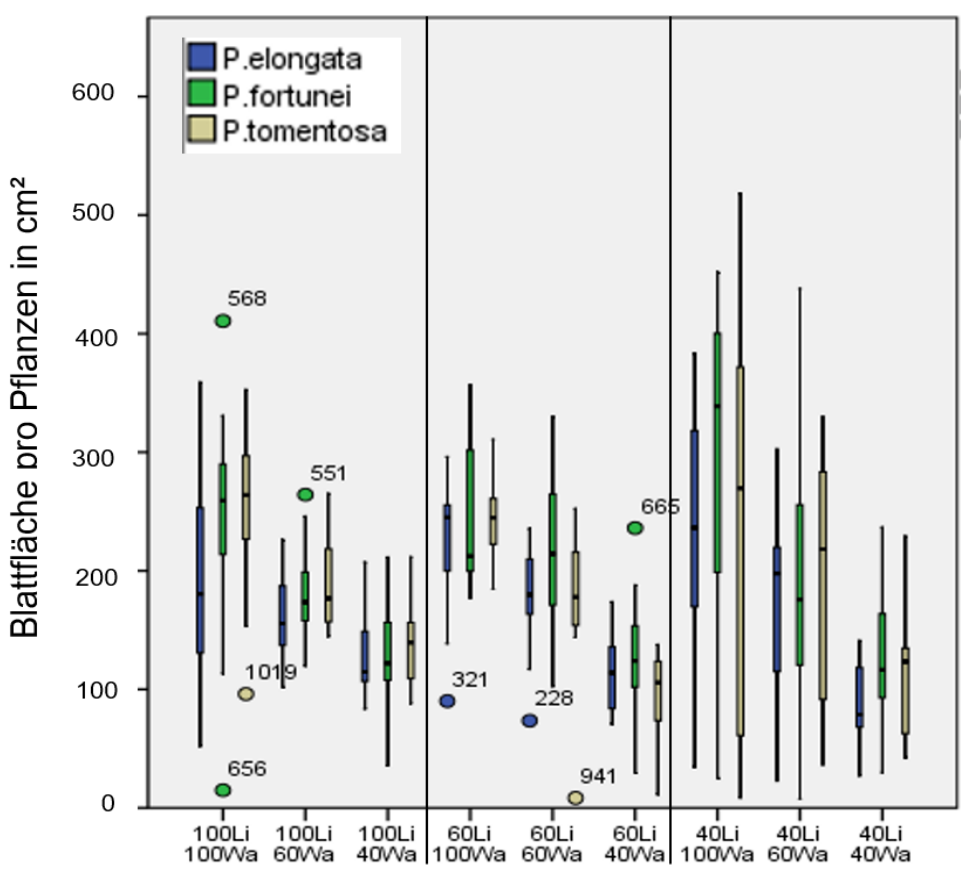

Abb. 41: Blattfläche pro Pflanze in $\mathrm{cm}^{2}$ unterteilt nach Behandlungsstufen (Licht und Wasser) und aufgeschlüsselt nach Paulownia-Arten. bzw. werfen die älteren schon vorhandenen Blätter nicht ab. Eine verminderte Lichtzufuhr führt folglich zu einer höheren Blattanzahl.

Um das Bild vervollständigen zu können, bietet sich eine Betrachtung der Gesamtblattfläche pro Pflanze an (Abb. 41).

Hierbei zeigt sich, dass weniger Wasser erwartungsgemäß auch eine geringere Blattfläche pro Pflanze zur Folge hat.

Im Gegensatz dazu wird mehr Blattfläche gebildet, wenn eine hohe Wassermenge zur Verfügung steht (100\%), aber die Lichtzufuhr abnimmt.

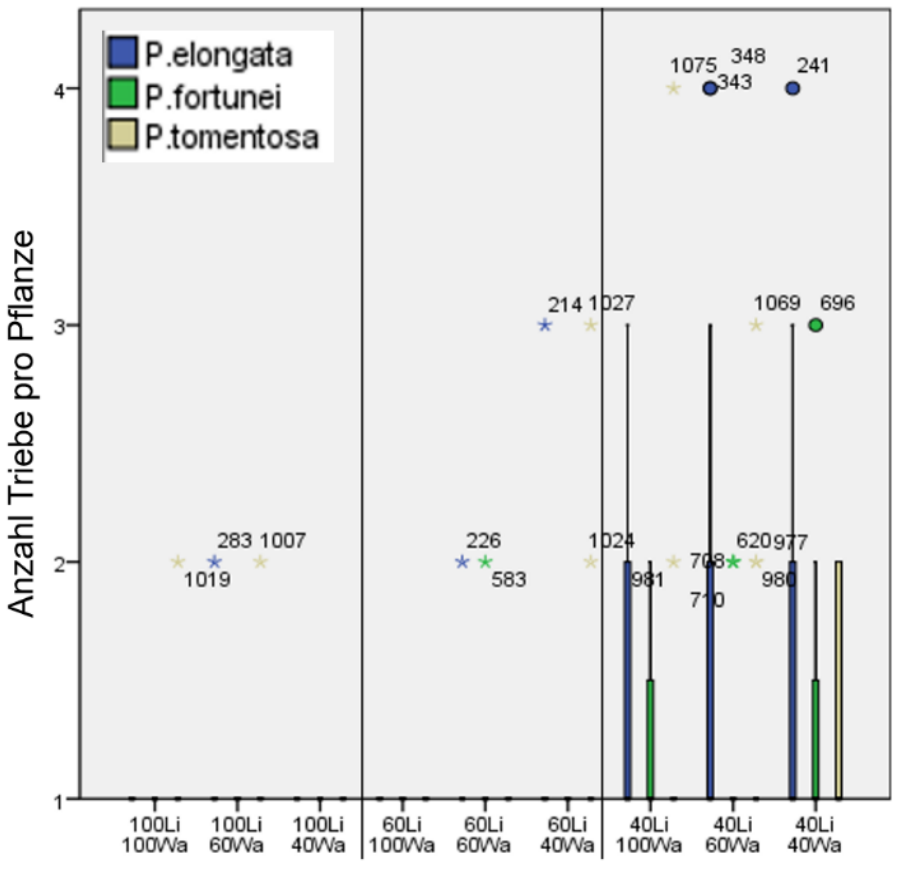

Abb. 42: Durchschnittliche Anzahl von Trieben pro Pflanze unterteilt nach Behandlungsstufen (Licht und Wasser) und aufgeschlüsselt nach Paulownia-Arten.

Die zusätzliche Ausbildung von Seitentrieben (Abb. 42) ist bei den Jungpflanzen in den ersten zwei Vegetationsperioden eher unüblich, da die Kronenbildung erst später einsetzt, und die Pflanze ihre Energie in der Jugendphase bevorzugt in einen Terminaltreib investiert.

Jedoch ist bei einer Lichtstufe von $40 \%$ zu beobachten, dass die Pflanzen in der zweiten Vegetationsperiode zusätzliche Seitentriebe ausbilden, um die verminderte Lichtzufuhr auszugleichen. Da das Licht hier der limitierende Faktor bei der Ausbildung der Seitentriebe ist, bleibt es unerheblich wie viel Wasser zur Verfügung steht. 


\subsection{Diskussion der Ergebnisse}

\subsubsection{Freilandanbau}

Um die Wachstumsreaktionen der der P.-Arten (P.elongata, P.fortunei und P.tomentosa) unter mitteleuropäischen Klimabedingungen beobachten zu können, wurden drei unterschiedliche Standorte, zum experimentellen Anbau im Freiland, ausgewählt.

Bei Brandenburg handelt es sich um einen zuvor landwirtschaftlich bearbeiteten Braunerde-Podsol Boden mit trockenen und nährstoffarmen Sanden. Das Kontinentalklima ist hier vorherrschend und bringt zumeist winterliche, sehr kalte Luftmassen aus dem Osten mit sich. Brandenburg entspricht in vielen Teilen vergleichsweise dem Klimabereich Nord- und Ostchinas, in welchem nahezu alle P.Arten wachsen können. Die an tropische Klimaverhältnisse gewohnten Arten, wie bspw. P.elongata, zeigen jedoch Wuchsprobleme bzw. Erfrierungen durch fehlende Verholzung (Zhu et al., 1986).

Die Versuchsfläche in Göttingen wurde vor dem Versuchsanbau jahrzehntelang als Lagerfläche für Bauschutt verwendet, schließlich aber stillgelegt und war dann jahrelang mit Gras überwachsen. Der Göttinger Standort bietet an Bodenverhältnissen eine Rendzina aus Kalkgestein im Wechsel mit einer flachgründigen Braunerde. Obwohl hier von einem Übergang zwischen atlantischem zu kontinentalem Klima gesprochen werden kann, ist der Göttinger Raum, durch seine Nähe zum Mittelgebirge Harz, doch sehr atlantisch beeinflusst. Ein Vergleich zu den heimischen Klimaten der Paulownia ist schwierig, entspricht aber in etwa Mittel- und Ost-China. Folglich war zu vermuten, dass die P.-Arten in Göttingen vergleichsweise am besten, auf das Klima bezogen, gedeihen würden.

Als Kontrast bzw. Erweiterung bot sich ein raues, klar atlantisch beeinflusstes Seeklima, wie es sich auf der Nordseeinsel Föhr findet, an. Dort vorherrschend sind Marschböden mit Podsol-Parabraunerden und sandigen Deckschichten über Geschiebelehm. Die Bodenverhältnisse sind denen in Brandenburg zumindest ähnlich und sollten ein gutes Wachstum der P.-Arten ermöglichen. Klimatisch lässt sich nur schwerlich ein Vergleich mit China herstellen, wobei es sich klar um einen Küstenstandort mit starken Winden handelt, der aufgrund des vorherrschenden Klimas eher nord-ost-chinesischen Verhältnissen zugeordnet werden kann.

Die Versuchsstandorte bieten folglich das Potential für sehr unterschiedliche Wachstumsreaktionen der verschiedenen P.-Arten, wobei klimatische Ähnlichkeiten zum natürlichen Verbreitungsgebiet in China durchaus gegeben und gewollt sind.

\subsubsection{Witterungsverhältnisse}

Wie die Auswertung der Daten des Deutschen Wetterdienstes zeigt, unterscheidet sich die Verteilung des Niederschlags auf den einzelnen Versuchsflächen lokal stark vom langjährigen Mittelwert, welcher in diesem Fall einen Bezugszeitraum von 30 Jahren umfasst $(1980-2010)$. 
In den Beobachtungsjahren von 2015 bis 2017 kam es innerhalb der Vegetationsperioden zu deutlich erkennbaren Frühjahrs- und Sommertrockenheiten und dass obwohl über das jeweilige Jahr verteilt der Gesamtniederschlag in Bereichen von 74 bis $140 \%$ zum langjährigen Mittel schwankte. Allerdings verschiebt sich die Verteilung dieses Niederschlages von der Vegetationszeit weg und in die Herbst- und Wintermonate hinein. Parallel dazu kann ein Temperaturanstieg beobachtet werden, der, über das Jahr gemittelt, durchschnittlich zwischen 0,6 bis $0,9^{\circ} \mathrm{C}$ liegt.

Eine höhere Temperatur innerhalb der Vegetationszeit ist den P.-Pflanzen laut Zhu et al. (1986) eher zuträglich, wobei das Optimum von $24-29^{\circ} \mathrm{C}$ nur an wenigen Sommertagen in Deutschland erreicht wird. Damit einhergehend erhöht sich natürlich auch der Wasserbedarf der Pflanzen, der aber wiederum von der Häufigkeit und der Menge der Niederschläge begrenzt wird.

Bei häufigeren Dürreereignissen innerhalb der Vegetationszeit und höheren Durchschnittstemperaturen verstärkt sich der Wasserstress für die Pflanzen zusätzlich, was sich negativ auf das Wachstum auswirkt (Abb. 43) (WiBei, 2008). Die gerade in der Anwuchsphase ungünstigen Witterungsverhältnisse im Versuchszeitraum haben negativen Einfluss auf die Überlebensrate der Jungpflanzen genommen und somit für hohe Ausfallraten gesorgt.

Auf eine Frühjahrstrockenheit reagieren die Pflanzen mit Verzögerung ihres Austriebs, was ihre Vegetationsperiode zusätzlich verkürzt. Der daraus resultierende zeitliche Wachstumsrückstand kann auch durch einen möglicherweise verstärkten Sommerniederschlag, wie es bspw. für Brandenburg und Göttingen im Jahr 2015 und 2017 der Fall ist, nicht mehr ausgeglichen werden.

Bei einem Vergleich der P.-Arten zeigt sich, dass P.elongata auf beiden Versuchsflächen die Trockenheit in der Anwuchsphase am besten übersteht und nur eine geringe Ausfallrate aufweist. Im Gegensatz dazu reagiert P.fortunei mit besonders hohen Ausfällen auf die Frühjahrstrockenheit, wie sie bspw. in Göttingen in allen drei Versuchsjahren auftritt.

Generell lässt sich feststellen, dass bei einem um ca. $30 \%$ trockeneren Frühjahr, als es im langjährigen Mittel üblich wäre, mit einer Ausfallrate der P.-Pflanzen im Bereich zwischen $20-30 \%$ zu rechnen ist.

Verstärkt sich diese Trockenheit auf über $40 \%$ des langjährigen Mittelwertes so schwanken die Ausfallraten der P.-Pflanzen in Bereichen von 20 bis $70 \%$ in Abhängigkeit von der jeweiligen Baumart.

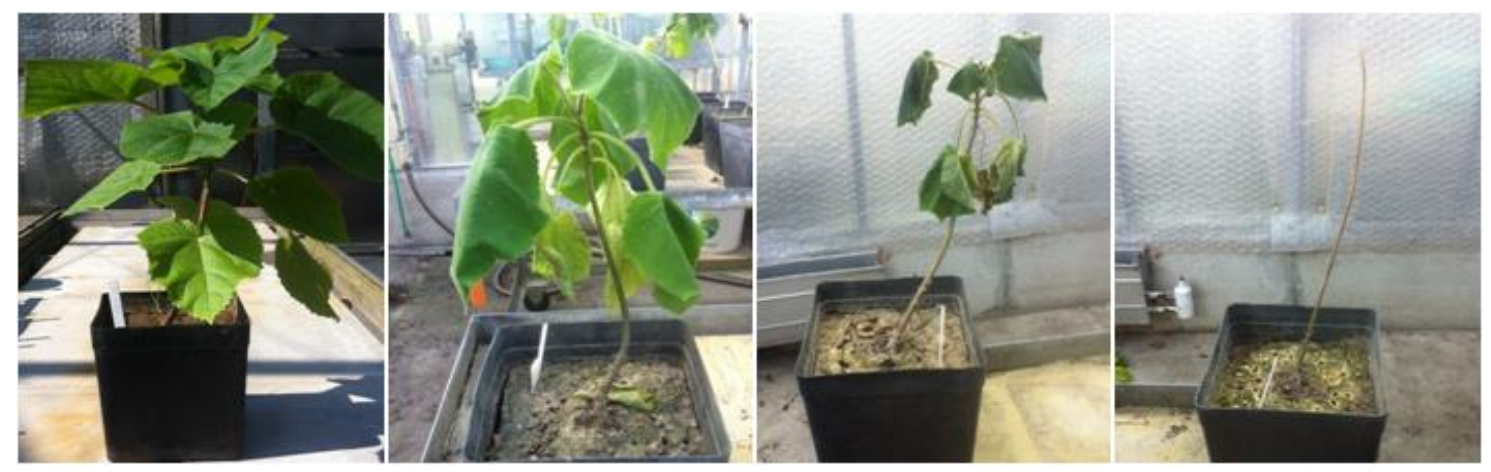

Abb. 43: Vitalitätsstufen nach Visnjic (2006), v.I.n.r.: Stufe $1=$ vitale Pflanze, Stufe 2 = mäßig vitale Pflanze, Stufe 3 = gering vitale Pflanze, Stufe 4 = vertrocknete Pflanze; verändert nach Büker (2015). 
Auf der Versuchsfläche in Brandenburg folgt im Jahr 2016 auf ein eher feuchtes Frühjahr eine starke Sommertrockenheit, was den Pflanzen große Probleme bereitet und die hohen Ausfallzahlen am Ende der Vegetationszeit erklärt.

Generell begegnen die Pflanzen einer Sommertrockenheit zuerst mit einem starken Welken und schließlich mit dem Abwurf der vergilbten Blätter, in der Reihenfolge von unten nach oben, also von den älteren hin zu den jüngeren Blättern (Abb. 44). Erst als allerletzte Maßnahme vergeht auch die Terminalknospe. Die dann evtl. nach einem Niederschlagsereignis wieder neuausgebildeten Lateral-Knospen (sympodiale Verzweigung) bilden Blätter aus, die von ihrer Oberfläche her deutlich kleiner sind, als es normalerweise in diesem Entwicklungsstadium der Fall wäre.

Es gilt der allgemeine Grundsatz, dass nur mit einer möglichst großen photosynthetisch aktiven Oberfläche, also möglichst vielen großen Blättern, die Einzelpflanze viel Biomasse erzeugen kann (Zhu et al., 1986). Da die dafür nötigen Blätter, aus den beschriebenen Gründen, nicht gebildet bzw. zwischenzeitig abgeworfen werden, bleibt die Paulownia hinter ihren, aus den heimischen Gefilden bekannten, Wuchserwartungen zurück

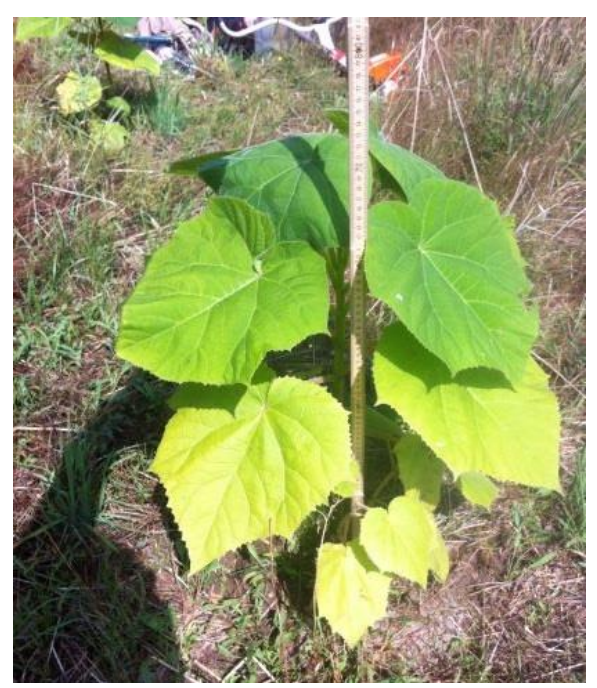

Abb. 44: Welken der Paulownia-Blätter durch Trockenstress, auf der Versuchsfläche in Brandenburg. (siehe auch Bork et al., 2015).

\subsubsection{Bodenbearbeitung und Bodenverhältnisse}

Die Paulownia-Keimlinge und die sich daraus entwickelnden Jungpflanzen sind vergleichsweise konkurrenzschwach (Bonner, 1990). Sie brauchen zur optimalen Entwicklung das volle Freilandlicht, da sie schon bei geringer Überschirmung durch Konkurrenzvegetation, in ihrem Wachstum zurückbleiben (Cathaia, 2020).

Beim Freilandanbau waren ähnliche Reaktionen zu beobachten und das, obwohl auf allen drei Versuchsflächen eine Bodenbearbeitung, in Form von Abstechen und Abtragen der Grasnarbe bzw. Umgrubbern der Gesamtfläche vor der Pflanzung, durchgeführt wurde. Auch eine in den Folgejahren regelmäßige Begleitwuchsregulierung konnte eine zeitlich begrenzte negative Beeinflussung des Wachstumsverlaufes der Pflanzen nicht ganz verhindern. Bork et al. (2015) haben ihre Freilandversuche in Bayern mit drei verschiedenen Pflegestufen (keine, mittel, hoch) behandelt und kamen dabei zu ähnlichen Erkenntnissen.

Neben der empfehlenswerten Bodenbearbeitung vor der Pflanzung haben auch die Bodenart und die damit verbundenen Bodeneigenschaften Einfluss auf den Anwuchserfolg. Speziell die Wasserspeicherkapazität der Böden kann hierbei entscheidend sein. 
Bei der Rendzina auf Kalkgestein bzw. der flachgründigen Braunerde in Göttingen zeigte sich, dass die Jungpflanzen, aufgrund der meist nur $20 \mathrm{~cm}$ Bodentiefe, zu wenig Wasser zur Verfügung hatten und kein ausreichendes Wurzelwerk ausbilden konnten (Longbrake, 2001). Es war ihnen also nicht möglich, über den natürlichen Niederschlag (50 - $90 \mathrm{~mm}$ nutzbare Feldkapazität) hinaus, sich in tieferen Bodenschichten einen Zugang zum Grundwasser zu erschließen.

Zhu et al. (1991) sind der Ansicht, dass das Grundwasser frühestens ab einer Tiefe von $1,5 \mathrm{~m}$ anstehen sollte, was die Pflanzen in ihrem natürlichen Verbreitungsgebiet im ersten Jahr ihrer Pflanzung zumeist erreichen. Sollte das Grundwasser flacher anstehen, so wirke sich dies ebenfalls negativ auf die Wurzelentwicklung aus.

Ein ähnliches Bild wie in Göttingen ergab sich auch in Brandenburg, wobei zu erwarten wäre, dass auf dem Braunerde-Podsol Boden eine gute Durchwurzelung leichter möglich ist. Allerdings reichte auch hier die nutzbare Feldkapazität, mit einem mittleren Bereich von 90 - $140 \mathrm{~mm}$ im effektiven Wurzelraum, für viele Jungpflanzen nicht aus, um mehrwöchige Dürreperioden, gerade in der empfindlichen Anwuchsphase, zu überstehen.

Die Pflanzen reagieren auf diesen wiederkehrenden Trockenstress mit dem Verwelken und schließlich mit dem Abwurf von Blättern. Die Verringerung der für die Photosynthese wichtigen Oberfläche führt zu Einbußen, sowohl beim Längenwachstum als auch in der Ausbildung des Wurzelwerkes, was im weiteren Verlauf der Vegetationsperiode nicht wieder ausgeglichen werden kann. Wenn sich dieser Kreislauf wiederholt, dann verlieren die Pflanzen von Mal zu Mal mehr an Kraft und sterben schließlich ab.

Ergänzend hierzu lässt sich, mit der frühzeitigen und ungewollten Aufgabe des Föhrer Versuchsanbaus, der Blickwinkel auf die komplett andere Seite verschieben. Es kann die Aussage von Zhu et al. (1986) bestätigt werden, wonach die P.-Pflanzen keine Staunässe vertragen und bereits nach drei Tagen der Absterbeprozess beginnt. Auf Föhr wurde im ersten Jahr der Pflanzung die regelmäßige Reinigung der Entwässerungsgräben, seitens der Gemeinde, vernachlässigt, weswegen die Pflanzen nach einem starken Niederschlagsereignis für mehrere Wochen überflutet wurden. Im Anschluss daran ist keine der Pflanzen mehr ausgetrieben.

\subsubsection{Behandlungsart}

Das Wachstum einer Pflanze wird durch sehr viele Faktoren beeinflusst, wobei bspw. der Pflanzentyp, die Standortsverhältnisse und die Art der Behandlung bei den beschriebenen Experimenten näher betrachtet wurden.

Auf den drei Versuchsflächen wurden die drei P.-Arten gleichmäßig in jeweils vier verschiedene Behandlungsarten unterteilt (siehe hierzu auch Mehrotra, 1997, 1996; Mehrotra et al., 1998). Die Pflanzlöcher von jeweils einem Viertel der Pflanzen wurden entweder mit Geohumus (G), mit Mykorrhiza-Substrat (M) oder mit der Kombination aus beidem (MG) behandelt. Das verbleibende Pflanzen-Viertel wurde unbehandelt eingepflanzt, um einen Referenzwert zu erhalten, also eine Kontrollgruppe ( $\mathrm{N}=$ Nullfläche).

Mittels einer multifaktoriellen Varianzanalyse (ANOVA) wurden die verschiedenen Variablen getestet und das Ergebnis, bezogen auf die untersuchten ersten drei Vegetationsperioden, war immer gleich. Die verschiedenen Behandlungsarten haben keinen statistisch nachweisbaren Einfluss auf das Höhenwachstum der P.-Pflanzen genommen. 
Nur zwischen den Standorten lässt sich ein Unterschied bereits zum Ende der zweiten Vegetationsperiode erkennen, der aber auch erst ab der dritten Vegetationsperiode signifikant wird.

Mehrere Gründe können ursächlich für das Ausbleiben von nachweislichen Reaktionen auf die verschiedenen Behandlungsarten sein:

- Die Menge an Geohumus bzw. Mykorrhiza-Substrat, welches in die Pflanzlöcher gegeben wurde, orientierte sich an der seitens des Herstellers empfohlenen Dosis in Abhängigkeit vom Bodenvolumen bzw. der Größe des Pflanzloches. Trotzdem kann es natürlich beim Pflanzvorgang zu Verschiebungen bzw. Verschüttungen der Substrate in tiefere Bodenschichten gekommen sein, wodurch sich ihre Wirkung evtl. nicht wie vorgesehen entfalten konnte (Visnjic, 2006). Allerdings wurden die Pflanzungen von einem professionellen Forstwirt durchgeführt, was die eigentliche Fehlerquote begrenzt.

- Auch wurde im Zusammenhang mit dem Mykorrhiza-Substrat vorab kein steriles Pflanzenmaterial verwendet. Die Pflanzenkeimlinge hatten also bereits in der Baumschule (Cathaia, 2020), aufgrund der dort vorherrschenden Bedingungen im Pflanzbeet, Kontakt mit Mykorrhiza, mit denen sie dann auch umgepflanzt wurden. Ein früherer Gewächshausversuch hatte das Vorhandensein von solchem Mykorrhiza-Geflecht an den Pflanzenwurzeln nachgewiesen (Büker, 2015). Inwiefern dann eine zusätzliche Mykorrhizierung des Pflanzloches noch zusätzliche positive Effekte mit sich bringt, bedarf des Nachweises durch einen weiteren, auf diesen Zweck spezialisierten Versuchsaufbau.

- Die durch den Einsatz der Substrate erhofften Wachstumseffekte können durch die hohen Ausfallraten verwischt bzw. überlagert worden sein. Die Stichprobenumfänge der unterschiedlichen Behandlungsarten sind schon zu Beginn der zweiten Vegetationsperiode, hervorgerufen durch die hohen Ausfallzahlen, sehr verschieden voneinander und bestehen in der dritten Vegetationsperiode zu Teilen nur noch aus Einzelpflanzen, was eine statistische Aussage beim Vergleich der Werte sehr schwächt.

- Natürlich ist es auch möglich, dass der Beobachtungszeitraum, mit insgesamt drei Vegetationsperioden, zu knapp bemessen war, um mögliche Effekte nachweisen zu können. Auch diesen Punkt kann nur ein erneuter und längerer Versuchsaufbau abschließend klären (siehe hierzu auch Bork et al., 2015).

Es ist aber auch möglich, dass die verwendeten Substrate einfach nicht die seitens des Herstellers versprochene Wirkung haben und daher nicht den erhofften Einfluss auf die Entwicklung der P.-Pflanzen nehmen (Geohumus, 2020; Mehrotra, 1997a). In dem Gewächshausversuch von Büker (2015), der sich einer ähnlichen Fragestellung widmete, konnten ebenfalls keine Entwicklungseffekte nachgewiesen werden.

\subsubsection{Pflanzenwachstum}

Um einen möglichst geraden Stamm zu erziehen, ist es beim Anbau von Paulownia üblich, die Pflanzen zum Ende der ersten Vegetationsperiode auf den Stock zu setzen (Wang and Shogren, 1992; Zhu et al., 1991, 1986). Dieser Empfehlung wurde auch auf den Versuchsflächen gefolgt und somit handelt es sich bei dem Längenwachstum in der zweiten Vegetationsperiode um einen vollständigen Neuaustrieb. 
Bereits am Ende der ersten Vegetationsperiode sind Unterschiede im Längenwachstum zwischen den beiden verbliebenen Versuchsstandorten in Brandenburg und Göttingen zu erahnen. Die Spannweiten der Brandenburger Werte sind deutlich größer als die der Göttinger, was sich von Vegetationsperiode zu Vegetationsperiode weiter verstärkt. Ursächlich hierfür sind die große Heterogenität im Längenwachstum der Brandenburger und die vergleichsweise hohe Homogenität der Göttinger P.-Pflanzen.

Alle Pflanzen in Göttingen trieben jedes Jahr neu vom Boden aus und erreichten dabei immer wieder ähnliche Höhen, weswegen sie sich nicht stark voneinander unterscheiden. Anders verhält es sich in Brandenburg, wo zu Beginn der dritten Vegetationsperiode ein Teil der P.-Pflanzen, aus den in der zweiten Vegetationsperiode ausgebildeten Stämmen, weiterwächst und nur wenige Pflanzen vom Boden neu austreiben. (siehe Bork et al., 2015)

Es ist zu vermuten, dass das um 20 \% feuchtere Frühjahr 2016 in Brandenburg den Pflanzen bei ihrem Neuaustrieb einen Wuchsvorsprung gegeben hat (Abb. 45). Durch die sich anschließende Sommertrockenheit, mit nur $50 \%$ des normalen Niederschlages, sind die Ausfallzahlen verständlicherweise sehr hoch. Trotz dessen konnte ein Teil der überlebenden Pflanzen zum Ende der Vegetationsperiode verholzen und die kalte Winterzeit ohne Zurückfrieren bzw. Austrocknung überstehen. Aus diesen Pflanzenstängeln konnten sie dann, im trockenen Frühjahr 2017 bei nur $60 \%$ des mittleren Niederschlages, wieder austreiben und sich zu größeren Pflanzen entwickeln, als im Vergleich zu Göttingen. Es gibt also eine Höhe ab der die Einzelpflanze nicht mehr zurücktrocknet, sondern weiterwächst und somit dem Schattendruck der Konkurrenzvegetation langsam entwächst. Bork et al. (2015) berichten von Rücksterbelängen des Haupttriebes in Bereichen von $40-60 \mathrm{~cm}$, was bei diesen Versuchen oft sogar der gesamten Pflanzenhöhe entsprach.

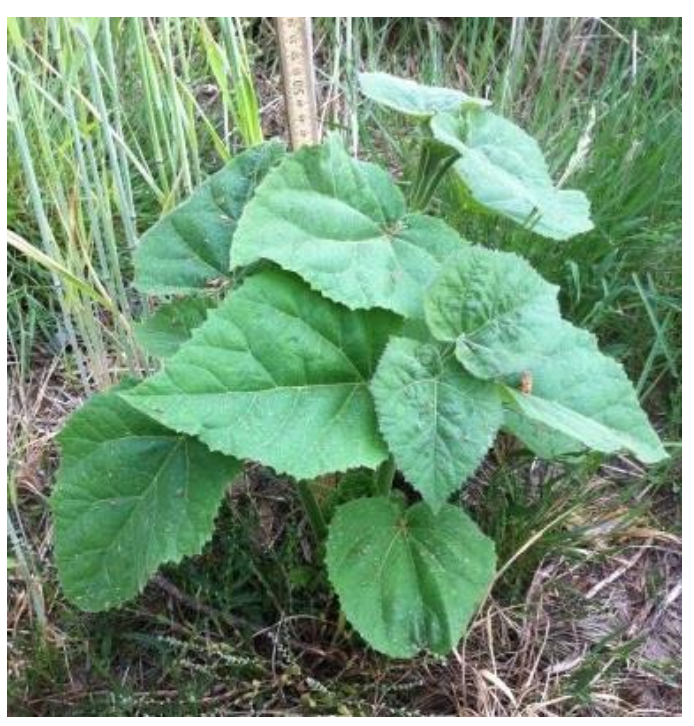

Abb. 45: Paulownia-Pflanze mit einer Höhe von $45 \mathrm{~cm}$ im Alter von 6 Monaten auf der Versuchsfläche in Brandenburg.

Ein signifikanter Unterschied im Wachstum der drei P.-Arten konnte nicht nachgewiesen werden. Zum einen war die Datengrundlage aufgrund der zu hohen Ausfallzahlen zu gering. Zum anderen konnte sich, bei einem solch geringen Wachstumsverlauf innerhalb der ersten drei Vegetationsperioden, ein phänotypischer Unterschied zwischen den Pflanzenarten nicht ausreichend herausbilden. Hierfür wären aller Voraussicht nach ein stärkeres Wachstum oder ein längerer Beobachtungszeitraum nötig gewesen.

Als zu Beginn der vierten Vegetationsperiode die Begleitwuchsregulierung eingestellt wurde, zeigte sich die geringe Konkurrenzkraft der Paulownia deutlich. Auf der Versuchsfläche in Göttingen sind auch die letzten Pflanzen nach einem erneuten Austrieb unter dem Druck der Konkurrenzvegetation eingegangen. In Brandenburg haben sich einzelne Pflanzenindividuen zu Jungbäumen herausgebildet, wobei sie von der Anzahl her weniger als $10 \%$ der ursprünglichen Anpflanzung entsprechen und sie bezüglich ihres Höhenwachstums weiterhin hinter den Erwartungen zurückbleiben (siehe Bork et al., 2015). 


\subsubsection{Gewächshausversuche}

Aufbauend auf den Freilandversuchen und zur Bearbeitung ergänzender Fragestellungen, wurde im Frühjahr 2016 ein zweijähriger Gewächshausversuch in Göttingen konzipiert. Hierbei sollte die Wechselwirkung von Licht und Wasser auf das Jugendwachstum - also der Übergang vom Keimling zur Jungpflanze - beobachtet werden.

Dabei wurden jeweils 180 Pflanzenkeimlinge der drei P.-Arten: P.elongata, P.fortunei und P.tomentosa verwendet. Das für die Paulownia übliche auf den Stock setzen, am Ende der ersten Vegetationsperiode, bot zusätzlich die Möglichkeit zur Beerntung der Stängel-Biomasse. Dieses Verfahren wurde folglich auch am Ende der zweiten Vegetationsperiode wiederholt.

Eine Abstufung des Lichtes wurde durch Schattennetze in den Stufen $60 \%$ und $40 \%$ erreicht. Hierbei orientieren sich die $100 \%$ Licht an dem zur Verfügung stehenden natürlichen Freilandlicht, welches durch das Glas der Gewächshauskonstruktion einfällt. Die Bewässerung der Pflanzen wurde händisch durchgeführt und in gleicher Abstufung unterteilt. Der $100 \%$ Wasseranteil und die Anzahl der Niederschlagsereignisse orientieren sich dabei an dem langjährigen Mittelwert des natürlichen Niederschlags in Rheinland-Pfalz, während der Vegetationszeit (Tab. 4: Wassermengen der drei Bewässerungsstufen.).

Bei Betrachtung der vorgestellten Ergebnisse zeigt sich, dass in der ersten Vegetationsperiode generell keine Reaktionen der Pflanzen auf die verschiedenen Behandlungsstufen zu beobachten sind. Erst zum Ende der zweiten Vegetationsperiode zeichnen sich Unterschiede nicht nur zwischen den Behandlungsstufen (100\%, $60 \%$ und $40 \%)$, sondern auch zwischen den Pflanzenarten ab. Hierbei nehmen die unterschiedlich hohen Ausfallraten Einfluss auf die Ergebnisdarstellung, was bspw. besonders bei der $40 \%$ Lichtstufe anhand der vielen Ausreißer-Werte erkennbar ist.

\subsubsection{Ausfallrate}

Unter Laborbedingungen und in Abhängigkeit von dem betrachteten Zeitraum kann es immer zu einem natürlichen Ausfall an Pflanzenindividuen kommen. Darüber hinaus nimmt selbstverständlich die Behandlungsart mit ihren verschiedenen Abstufungen direkten Einfluss auf die Überlebenswahrscheinlichkeit der Pflanzen.

Bezogen auf die erste Vegetationsperiode (1. VP) ähneln sich die relativ geringen Ausfallraten der beiden Behandlungsarten Licht und Wasser. In der zweiten Vegetationsperiode (2. VP) hingegen deutet sich ein größerer Effekt des Lichtes an.

Bei abnehmendem Licht, was gleichbedeutend ist mit zunehmender Beschattung, ergeben sich, über alle Behandlungsstufen und P.-Arten hinweg, in der 1. VP Ausfallraten von $2-10 \%$ und $7-49 \%$ in der 2 . VP.

Im Vergleich dazu fallen bei abnehmender Wasserverfügbarkeit zwischen 5-7\% in der 1. VP und $16-26 \%$ in der 2. VP an Pflanzen aus. Bei einer Verminderung des Lichtes zeigt sich folglich eine deutlich höhere Ausfallrate als es bei einer Verringerung der Wasserverfügbarkeit der Fall ist. 
Da die Pflanzen in der 1. VP als Keimlinge starten, ist ihre Empfindlichkeit gegenüber Beschattung und Wasserknappheit anfänglich noch gering, bzw. es steht jeweils mehr zur Verfügung, als in diesem frühen Stadium zur Entwicklung gebraucht wird. Trotz dessen sind erhöhte Ausfälle, bei einer $40 \%$ Beschattung, zum Ende der 1. VP bereits erkennbar.

Zu Beginn der zweiten Vegetationsphase treiben die Jungpflanzen aus dem etablierten Wurzelstock erneut aus, und ihre Entwicklung wird erkennbar am stärksten vom Licht begrenzt. Bei Betrachtung der Kombination von $100 \%$ Wasser und abnehmender Lichtverfügbarkeit am Ende der 2. VP scheint es im Bereich zwischen $60-40 \%$ Beschattung einen Schwellenwert zu geben, ab dem die Überlebenswahrscheinlichkeit für die Jungpflanzen kritisch wird. Die Ausfallraten summieren sich in hier von 5 auf 45 $\%$. In Abhängigkeit von der Wasserstufe ergeben sich bei $40 \%$ Lichtverfügbarkeit noch drastischere Ausfallraten in Höhe von $41-61 \%$.

Wird in Ergänzung dazu die umgedrehte Kombination aus $100 \%$ Licht und abnehmender Wasserverfügbarkeit betrachtet, dann zeigen sich Ausfallraten von nur 1 - $15 \%$, also um ca. $1 / 3$ geringer als bei den vergleichbaren Lichtwerten. Aber auch hier zeichnet sich wieder eine Schwelle im Übergang von 60 zu $40 \%$ Wasserverfügbarkeit $\mathrm{ab}$, die aber scheinbar weniger stark limitierend wirkt, als es beim Licht der Fall ist.

Einzig und allein bei der Kombinationsstufe von $100 \%$ Licht zu $60 \%$ Wasser ist in der 1. VP kein Ausfall zu verzeichnen. Damit einhergeht auch die gegenteilige Beobachtung, dass der höchste Ausfall sich am Ende der 1. VP bei $40 \%$ Licht und $100 \%$ Wasser zeigt. In dieser Konstellation reagiert P.elongata mit $30 \%$ Ausfall im Vergleich zu P.fortunei mit $0 \%$ am empfindlichsten.

In der 2. VP ist erwartungsgemäß in der Kombinationsstufe von $40 \%$ Licht und $40 \%$ Wasser die Ausfallrate mit $61 \%$ am höchsten. Hier bilden P.elongata mit $75 \%$ und P.fortunei mit $45 \%$ Pflanzenausfall die beiden gegensätzlichen Extreme ab, was verwundert, da laut Aussage von Zhu et al. (1986) P.fortunei am schattenintolerantesten von allen P.-Arten ist. In der Natur kann die Verjüngung von P.fortunei nur im Offenland auflaufen, das sie keine Beschattung übersteht (Zhu et al., 1986).

Über alle Behandlungsarten und -stufen hinweg erweist sich aber P.tomentosa mit den durchschnittlich geringsten Ausfallraten als die robusteste der P.-Arten unter den gegebenen Versuchsbedingungen.

Allerdings sind die Aussagen nur unter Vorbehalt zu treffen, da aufgrund von Platzmangel der Versuchsaufbau so arrangiert werden musste, dass die Pflanztöpfe auf den Tischen direkt nebeneinander standen. Dies Enge bedingt, dass sich die Pflanzen bereits ab einer Höhe von ungefähr $20 \mathrm{~cm}$ gegenseitig beschatten.

Diesbezüglich kann aber auch von einem natürlichen Beschattungseffekt gesprochen werden, wie er bei einer natürlichen Verjüngung im Freiland auftritt, bei der die Pflanzen noch näher nebeneinander keimen. Wie stark der Einfluss dieser natürlichen Beschattung auf das Wachstumsverhalten bzw. die Ausfallrate der Jungpflanzen ist, konnte im Rahmen der Gewächshausversuche nicht näher quantifiziert werden. 


\subsubsection{Längenwachstum}

Auch beim Längenwachstum lässt sich am Ende der 1. VP noch kein signifikanter Unterschied zwischen den Behandlungsarten und -stufen feststellen. Hierfür können die gleichen Gründe aufgeführt werden, wie sie vorab bereits erläutert wurden.

Erst in der 2. VP sind signifikante Unterschiede nachweisbar, wobei die Ergebnisse jedoch mit großen Spannweiten verbunden sind. Besonders sichtbar wird dies bei einer Betrachtung der Kombination von 40 \% Licht zu $100 \%$ Wasser anhand der zahlreichen Ausreißer-Werte.

In diesem Zusammenhang kann fast schon von einem zweischichtigen Bestand gesprochen werden. Dieser setzt sich aus Pflanzenindividuen zusammen, die ein starkes Längenwachstum zeigen und in die Höhe streben und anderen Pflanzen, die im Wachstum zurückbleiben, dadurch stark beschattet werden und nur noch wenig in der Länge zuwachsen.

Das Längenwachstum ist in der eben schon erwähnten Kombination von 40 \% Licht zu $100 \%$ Wasser am stärksten und lässt aber, mit Verminderung der Wasserverfügbarkeit auf 60 bzw. $40 \%$, kontinuierlich nach.

Im Gegensatz dazu fällt das Wachstum bei $100 \%$ Licht und $40 \%$ Wasser am geringsten aus. Die Pflanzen streben also bei wenig Licht verstärkt in die Höhe solange genügend Wasser verfügbar ist. Wenn das Verhältnis aber umgekehrt wird und das Wasser der limitierende Faktor ist, dann führt der volle Lichtgenuss zu keinem verstärkten Wachstum.

Generell lassen sich signifikante Unterschiede nur zwischen den Behandlungsstufen feststellen und nicht zwischen den drei P.-Arten.

Es ist jedoch erwähnenswert, dass bspw. P.elongata die größte Gesamtlänge in der Kombination von $40 \%$ Licht zu $100 \%$ Wasser mit $54 \mathrm{~cm}$ als Mittelwert in der 1. VP und $156,5 \mathrm{~cm}$ in der 2. VP zeigt. Dabei ist zu beachten, dass P.elongata bei dieser LichtWasser Kombination in der 1. VP eine Ausfallrate von $30 \%$ und in der 2. VP von $45 \%$ aufweist, was eine Verschiebung des Mittelwertes zur Folge hat. Im Vergleich dazu erklärt sich bei P.tomentosa, wegen der nur geringen Ausfallrate, die große Spannweite und ein Längenwachstum das geringer ausfällt als bei P.elongata.

\subsubsection{Durchmesserwachstum}

Das Wachstum des Durchmessers hat sich im Vergleich von der ersten zur zweiten Vegetationsperiode erhöht und dass, obwohl es sich hierbei um einen Neuaustrieb handelt. Dabei fällt der Zuwachs nicht im gleich hohen Maße aus wie es bspw. beim Längenwachstum zu beobachten ist.

Trotz dessen steht das Wachstum des Durchmessers auch weiterhin im Verhältnis zur Länge, wie eine Betrachtung des H/D-Verhältnisses (Höhe zu Durchmesser) zeigt.

Jedoch weist das H/D-Verhältnis in der 2. VP deutlich größere Werte und eine höhere Streuung auf. Diese beruht einerseits auf den vielen Pflanzen, die im Längenwachstum zurückgeblieben sind und jetzt beschattet werden und nur noch wenig in der Länge, aber verstärkt im Durchmesser zuwachsen. 
Anderseits gibt es auch einige Pflanzen die vergleichsweise stark in der Länge zugewachsen sind, wobei sich ihr Durchmesser aber nicht proportional dazu mitentwickelt hat. Für die Autoren Zhu et al. (1986) ist das richtige H/D-Verhältnis eines der vier entscheidenden Kriterien zur Auswahl von Setzlingen im ersten Wuchsjahr. Sie empfehlen auf ein generelles H/D-Verhältnis von $60-70$, bzw. bei P.fortunei von ca. 60 zu achten, da dies eine stabile Pflanze garantiert. Bei der Mehrzahl der Gewächshauspflanzen schwankten die H/D-Verhältnisse am Ende der ersten VP in Bereichen von 20 bis 100 und nach der 2. VP zwischen 100 bis 200 , was auf eine geringe Stabilität schließen lässt.

Bei Betrachtung der verschiedenen Kombinationsmöglichkeiten der Behandlungsstufen stellt sich heraus, dass das Wasser der limitierende Faktor in der Durchmesserentwicklung ist und nicht das Licht. Erst bei einer geringen Lichtverfügbarkeit wie $40 \%$ haben auch die verschiedenen Wasserstufen keinen nachweislichen Einfluss mehr auf die Durchmesserentwicklung.

Anders als es bspw. beim Längenwachstum zu beobachten ist, zeichnet sich mit Abnahme des Lichtes keine signifikante Zunahme des Durchmessers ab.

\subsubsection{Trockenmasse}

Mit der Bezeichnung Trockenmasse ist generell nur die oberirdische Stängelbiomasse gemeint ohne die Blattbiomasse, die in diesem Falle nicht weiter beachtet bzw. erfasst wurde. Die oberhalb des Wurzelhalsdurchmessers abgeerntete Stängelbiomasse wurde, um sie vergleichen zu können, anschließend bei $105^{\circ} \mathrm{C}$ getrocknet und gewogen.

Bei Betrachtung des Stängels fällt der Hohlraum im Querschnitt auf, der selbst noch bei der Ernte des ausgewachsenen Baumes deutlich erkennbar ist (Abb. 46). Es handelt sich bei dem Stängel folglich um eine Röhre, dessen Loch in der Mitte sich im Querschnitt beim Dickenwachstum nur marginal vergrößert und im Grunde kein Problem bei der Verwendung des späteren Stammes darstellt.

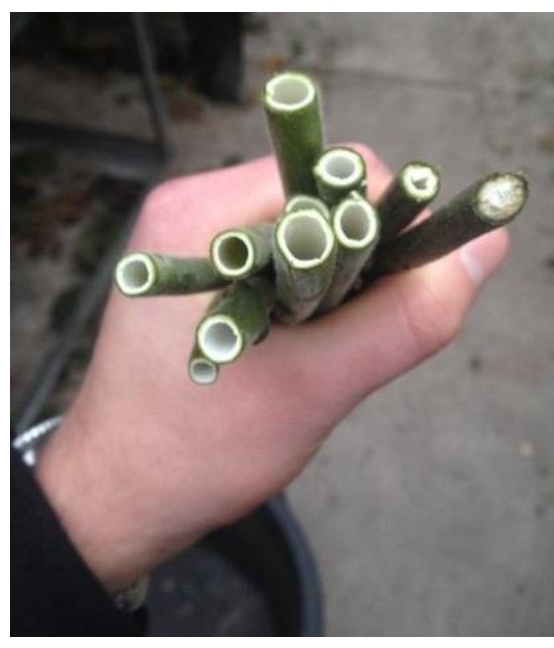

Abb. 46: Abgeerntete PaulowniaStängel aus dem Gewächshaus; erkennbar der Röhrenquerschnitt.

Generell lassen sich keine Unterschiede zwischen den P.-Arten bei der Ausbildung der Stängelbiomasse feststellen.

Bei den Behandlungsstufen, in den Kombinationen von 100 und $60 \%$ Licht zu 100 und $60 \%$ Wasser, ist kein signifikanter Unterschied zwischen den beiden Vegetationsperioden nachweisbar. Es wurde also, entgegen jeglicher Erwartung, in der 2. VP nicht mehr, sondern ähnlich viel bzw. stellenweise sogar weniger Stängelbiomasse gebildet und das auch trotz stärkerem Längen- und Dickenwachstum.

Bei der Kombinationsstufe von $40 \%$ Wasser und unabhängig von der Lichtzufuhr, wurde in der 2. VP sogar signifikant weniger Masse gebildet als in der 1. VP.

Bei Betrachtung der Höhe im Verhältnis zum Gewicht (ähnlich dem H/D-Verhältnis) zeigt sich in der 2. VP, dass nahezu die Hälfte aller Pflanzen zwar doppelt so groß (Längenwachstum) aber nur noch halb so schwer (Biomasse) sind. 
Bei gleichbleibend hoher Bewässerung von $100 \%$ und mit Abnahme der Lichtzufuhr verringert sich zwar sichtlich die Stängelbiomasse, doch die Werte sind nicht signifikant. Erst bei einer Kombination von 100 \% Licht und einer Verminderung der Bewässerung ist eine signifikante Abnahme in der 2. VP messbar.

Gleichzeitig sind die Spannweiten der P.-Arten, im Vergleich zu den Werten für die Länge und den Durchmesser, bei der Trockenmasse am geringsten ausgeprägt.

Am nächsten liegen die Messwerte bei der $40 \%$ Wasserstufe beieinander. Es findet folglich bei dieser $40 \%$ Schwelle nur eine geringe Ausdifferenzierung zwischen den Pflanzenindividuen statt.

Dies kann ein Hinweis darauf sein, dass in der 2. VP bei dieser Behandlungsstufe der Punkt erreicht ist, an dem die Pflanzen gerade noch überleben und kein zusätzliches Wuchspotential mehr aufbauen können.

Die Reaktionen der Pflanzen in der 2. VP sind eine direkte Folge der Behandlung von der 1. VP. Im Ergebnis hätten die Pflanzen in der 2. VP mehr Licht und mehr Wasser und somit indirekt mehr Wuchsraum gebraucht, als es innen der Versuchsaufbau ermöglicht hat, um bezüglich der Biomasse weiteren Zuwachs generieren zu können.

Natürlich könnte auch ein größerer Teil des Biomassezuwachses in zusätzlicher Blattbiomasse umgewandelt worden sein, was im Umfang dieses Experiments aber nicht weiter erfasst wurde.

\subsubsection{Blattfläche}

Zur Ermittlung der Blattanzahl bzw. Blattfläche wurde zum Ende der zweiten Vegetationsperiode, noch vor dem natürlichen Blattabwurf, eine Vollaufnahme durchgeführt, was in diesem Falle die Vermessung von über 5.000 Blättern beinhaltete. Im Ergebnis zeigt sich, dass sich zwischen den Behandlungsarten und -stufen deutlich stärkere Unterschiede bei der Blattfläche und weniger bei der Blattanzahl herausgebildet haben.

Bezüglich der Blattfläche sind signifikante Unterschiede zwischen den Behandlungsstufen aber nicht zwischen den P.-Arten nachweisbar. Auch hierbei sind wieder die Spannweiten der Messungen sehr groß, was indirekt auf die sehr heterogene Entwicklung der Pflanzenindividuen im Vergleich zueinander verweist.

Bei den 100 und $60 \%$ Lichtstufen sind die Unterschiede in der Anzahl der Blätter nur gering und das auch ganz gleich wie viel Wasser zur Verfügung steht. Erst bei einer sehr niedrigen Lichtzufuhr von nur $40 \%$ bilden die Pflanzen signifikant mehr Blätter aus, bzw. werfen die älteren nicht ab.

Noch stärker reagieren die Pflanzen mittels einer Zu - bzw. Abnahme der Blattfläche auf die verschiedenen Wasserstufen. Dabei bedingt generell weniger Wasser eine geringere Gesamtblattfläche pro Pflanze. Die größte Blattfläche wurde von den Pflanzen gebildet, die nur $40 \%$ Licht, aber $100 \%$ Wasser zu Verfügung hatten. Allerdings zeigen sich hierbei keine signifikanten Unterschiede zwischen den Lichtstufen, sondern nur zwischen den Wasserstufen. 
Bei einer Lichtstufe von $40 \%$ reagieren die Pflanzen in der zweiten Vegetationsperiode mit der Ausbildung von zusätzlichen Seitentrieben auf die geringe Lichtverfügbarkeit. Diese Reaktion ist bei Jungpflanzen in den ersten drei bis vier Vegetationsperioden eher unüblich, da die eigentliche Kronenbildung erst in späteren Jahren einsetzt, und die Pflanze ihre Energie in der Jugendphase verstärkt in einen Terminaltreib investiert (Zhu et al., 1986). Folglich ist die Ausbildung der Seitentriebe eine direkte Reaktion der Pflanze auf die verminderte Lichtverfügbarkeit. Dabei konnten allerdings keine Unterschiede zwischen den P.-Arten festgestellt werden.

\subsubsection{Resümee}

Wie die Freilandversuche gezeigt haben, ist ein Anbau von Paulownia unter mitteleuropäischen Klimabedingungen möglich. Es ist jedoch mit hohen Ausfällen zu rechnen, insofern es in den ersten Jahren der Pflanzung verstärkt zu Frühjahrs- bzw. Sommertrockenheit kommt. Der Anwuchserfolg ist folglich stark von den vorherrschenden Witterungsverhältnissen abhängig. Eine zusätzliche Bewässerung oder die Wahl von älteren Pflanzenindividuen (Heister) kann hier hilfreich sein, würde jedoch die Kosten eines Anbaus erhöhen (Cathaia, 2020; EnPf, 2021; WeGrow, 2020).

Neben dem Anwuchserfolg wird auch das Längenwachstum von den klimatischen und standörtlichen Bedingungen beeinflusst. $\mathrm{Zu}$ möglichen Unterschieden in den Wachstumsreaktionen und im Wachstumsverlauf zwischen den drei P.-Arten konnten die Experimente nur unzureichende Ergebnisse liefern. Dafür war das reale Wachstum der Pflanzen zu gering, da es weit hinter den Erwartungen, die mit diesen Baumarten im Allgemeinen verknüpft werden, zurückblieb. Außerdem waren die Ausfallzahlen unerwartet hoch und bedingten so einen vorzeitigen Abbruch des Freilandexperiments und eine Verkürzung des geplanten Beobachtungszeitraumes.

Die im Freiland getesteten Behandlungsarten, in Zusammenhang mit Geohumus und Mykorrhizierung, haben keinen nachweislichen Effekt auf das Wachstum der Pflanzen gehabt.

Allerdings könnten auch hierbei die hohen Ausfallzahlen mögliche Reaktionen in den Daten verwischt haben. Auch wäre ein längerer Beobachtungszeitraum hilfreich, um abschließende Aussagen zu den Hilfsstoffen treffen zu können, denn es gibt in der Literatur zahlreiche Hinweise auf deren positive Wirkungen (Mehrotra, 1996; Mehrotra et al., 1998; Mehrotra, 1997a).

Auch für den Nachweis weiterer arttypischer Unterschiede war das Wachstum zu gering bzw. der Beobachtungszeitraum zu kurz. Es ist zu erwarten, dass sich die typischen Unterschiede zwischen den P.-Arten erst in den Folgejahren herausbilden und sich diese auch von Vegetationsperiode zu Vegetationsperiode weiter verstärken würden.

Es hat sich gezeigt, dass das Wachstum der Pflanzen im Gewächshaus verschiedenartig von den gegebenen Licht- und Wasserverhältnissen beeinflusst wurde. 
Bei einer Verringerung des Lichts nehmen das Längen- und das Durchmesserwachstum der Pflanzen zu. Dabei entwickelt sich der Durchmesser aber nicht in gleicher Proportion wie die Länge. Dies führt zu einer Verschiebung des Verhältnisses der Höhe zum Durchmesser und hat längere, aber dünnere Pflanzen zur Folge, was bereits in der zweiten Vegetationsperiode erkennbar ist.

Eine Verringerung des Wassers nimmt, im Gegensatz zu einer Verringerung des Lichtes, keinen signifikanten Einfluss auf die Länge. Bei der Entwicklung des Durchmessers ist hingegen die Wasserverfügbarkeit der limitierende Faktor. Mit dem Rückgang der Wasserverfügbarkeit ist auch eine Verringerung der Stängelbiomasse und der Blattflächen verbunden.

Die Blattanzahl hingegen ist vom Licht abhängig. Erst bei starker Beschattung (40\%) erhöht sich die Anzahl der Blätter, bzw. die Pflanzen versuchen die vorhandenen Blätter so lange wie möglich zu erhalten.

Unterschiede in der Reaktion der drei P.-Arten auf die verschiedenen Behandlungsarten und -stufen konnten bei dem Gewächshausexperiment nicht nachgewiesen werden. Auch hierzu bedarf es voraussichtlich eines längeren Beobachtungszeitraumes über zwei Vegetationsperioden hinaus.

Die Gewächshausexperimente haben gezeigt, dass im Bereich zwischen $60-40 \%$ des natürlichen Lichtes eine Schwelle existiert, bei der es zu hohen Ausfallzahlen bei der Verjüngung bereits in der ersten und sich weiter verstärkend in der zweiten Vegetationsperiode kommt.

Sowohl die eigentliche Keimung als auch das spätere Heranreifen der Samen zu Jungpflanzen erfordert intensiven Lichtgenuss, da laut Zhu et al. (1986) bereits eine Beschattung von ca. $70 \%$ tödlich auf die Verjüngung wirken kann. Wie die Freilandversuche aber auch indirekt gezeigt haben, mindert der sich auf einer Freifläche, bspw. einem Windwurf, schnell ausbreitende Begleitwuchs das Wachstum der P.Verjüngung (siehe Bork et al., 2015). Es erscheint daher mehr als fraglich, ob Paulownia, aufgrund seiner geringen Schattentoleranz, das Potential für eine natürliche Verbreitung in mitteleuropäischen Wäldern besitzt. Erst recht wenn die Baumart ihr Wuchspotential nicht voll auszuschöpfen vermag. (Bork et al., 2015)

In den natürlichen Waldbeständen Chinas findet sich Paulownia meist nur einzelstammweise an exponierten Standorten mit großem Lichtangebot, was beispielsweise entlang von Flüssen oder offenen Tälern der Fall ist. Daher sind natürlich entstandene P.-Waldbestände selten und weisen dann auch nur eine geringe Stammanzahl auf, bzw. einen niedrigen Bestockungsgrad.

Sollte die Baumart in Mischung mit anderen Arten vorkommen, so ist auch das nur möglich, wenn Paulownia eine gleichgroße oder größere Kronenhöhe aufweist, als die sie umgebenden anderen Baumarten. Die Autoren Zhu et al. (1986) schlussfolgern daher, dass Paulownia eine Pionierbaumart ist, die sich nicht auf natürliche Weise in Wäldern verjüngen kann, sondern dafür immer Brachen, Kahlschläge oder ehemalige Waldbrandflächen benötigt. 


\subsubsection{Anbauwürdigkeit}

Wie die Freiland- und Gewächshausversuche verschiedenartig gezeigt haben, ist der Anbau von Paulownia möglich, jedoch bedarf die Frage nach der Anbauwürdigkeit einer etwas differenzierteren Betrachtung.

Einmal abgesehen von den typischen Vorgärten sind die in Deutschland zurzeit denkbaren Landnutzungsysteme zum aktiven Anbau von Paulownia Kurzumtriebsplantagen und Agroforstsysteme.

\subsection{P.-Anbau auf Kurzumtriebsplantagen}

Ein P.-Anbau auf Kurzumtriebsplantagen (KUP) findet in Deutschland bereits statt und wird von Firmen wie bspw. der Baumschule Cathaia (2020) als Geschäftsmodell angeboten und mit Bewirtschaftungsempfehlungen unterstützt (siehe auch WeGrow, 2020).

KUP können in den historischen Kontext als eine Spezialisierung bzw. moderne Weiterführung der altbewährten Bewirtschaftungsart des Niederwaldes eingeordnet werden (Dickmann, 2006; Meyerhof, 2014; Setzer, 2019).

Alle aufgeführten Definitionen einer KUP, sei es nun nach Thomasius (1991) oder nach Knust (2009) stehen einer Erweiterung der bestehenden Baumartenpalette um die Gattung Paulownia nicht im Wege. Ganz im Gegenteil besitzt die Gattung eine ganze Reihe von vorteilhaften Eigenschaften, die sie besonders für den Anbau in Niederwälder bzw. auf Plantagen im Kurzumtrieb empfehlen, wie Zhu et al. (1991, 1986) ausführlich darlegen (siehe auch Hofheinz, 2016).

Natürlich braucht es dafür eine Anpassung des rechtlichen Rahmens, um Paulownia in die Liste der förderfähigen KUP-Baumarten aufnehmen zu können (VwG Köln, 2014). Nach Böhm et al. (2017) sind bisher mehrere Baumarten auf ein und derselben Fläche sowieso nicht als Dauerkultur förderfähig, was indirekt auch für den Anbau von Paulownia sprechen würde, da sie sich aufgrund ihrer geringen Schattentoleranz nicht mit anderen Baumarten verträgt (Zhu et al., 1986).

Bei der klassischen Anlage einer KUP braucht es für die Paulownia keine Extraplanung, da sie ganz typisch in Reihen gepflanzt werden kann. Dabei wäre sie bezüglich des Pflanzverbandes, der sich in Bereichen von $5 \times 4 \mathrm{~m}$ (500 Pflanzen / ha) bis zu $6 \times 12 \mathrm{~m}$ (138 Pflanzen / ha) erstrecken kann, sehr flexibel einsetzbar (Zhu et al., 1986). Des Weiteren unterscheidet sie sich in Punkto Pflanzverfahren und Pflege nicht von den üblichen KUP-Gehölzen.

Die Paulownia erfüllt viele der allgemeinen Anforderungen, die laut Schildbach et al. (2009) an KUP-Baumarten gestellt werden, im besonderen Maße (siehe auch 2.2.2.2.3 Paulownia als mögliche KUP-Baumart):

- Sie ist problemlos vermehrbar (generativ wie vegetativ),

- zeigt ein besonders rasches Jugendwachstum (Pionierbaumart),

- hat dabei eine hohe Biomasseleistung, wobei

- bisher nur geringe Anfälligkeiten gegenüber abiotischen (Richter and Böcker, 2001; Stimm et al., 2013) und biotischen (Cabi, 2019; Mehrotra, 1997b) Schäden bekannt sind (siehe Hao et al., 2004) und

- $\quad$ sie besitzt ein hohes Stockausschlagvermögen (Zhu et al., 1986). 
Allerdings ist, wie die Freilandversuche gezeigt haben, eine Anwuchssicherheit bei Jungpflanzen nicht garantiert, und sie besitzen auch nur eine geringe Jugenddichtstandsverträglichkeit. Die Pflanzen sind sehr empfindlich gegenüber Staunässe, haben dafür aber eine hohe Trockenresistenz, wobei dies zumeist Einbußen bei der Biomasseproduktion mit sich bringt (Zhu et al., 1991, 1986).

Die Bewirtschaftung einer reinen Paulownia-KUP ist vergleichsweise einfach, da sich die Endprodukte sowohl zur stofflichen als auch energetischen Nutzung eignen, wie es Zhu et al. (1986) ausführlich beschreiben. Darüber hinaus ist auch der Anbau zur FurnierGewinnung möglich und von der ökonomischen Betrachtungsweise her aus auch erstrebenswert. Jedoch bedarf dies zumeist zusätzlicher Investitionen in Form von Pflegemaßnahmen zur Ästung der Bäume (siehe 1.1.5.2.2 Kronenwachstum).

Die naturschutzfachliche Bewertung einer mit P.-Baumarten begründeten KUP ist, wie das Kapitel II zeigt, schwierig, da auch sie ganz typisch für eine KUP nur einen geringen Natürlichkeitsgrad bei gleichzeitig hoher Bewirtschaftungsintensität besitzt. Generell entsteht durch die Neuanlage einer KUP, laut Schmidt und Glaser (2009), ein: „[...] neuartiges Ökosystem in der Landschaft mit Eigenschaften, die unvereinbar mit dem Naturschutz erscheinen [...]“. Natürlich verschärft sich diese Aussage noch in besonderem Maß, wenn eine nichtheimische Baumart verwendet wird. Allerdings werden hier KUP mit Gehölzökosystemen in Form von Wäldern verglichen, was nicht ganz widerspruchsfrei erscheint.

Wird hingegen ein Vergleich $\mathrm{zu}$ anderen landwirtschaftlichen Kulturen und Nutzungsformen vorgenommen, so besitzt eine Paulownia-KUP durchaus ein hohes Potential das Landschaftsbild zu bereichern und in ökologischer Hinsicht aufzuwerten.

\subsection{P.-Anbau in Agroforstsystemen}

Die jahrhundertealte, mannigfaltige Kombination von Pflanzen, Sträuchern und Gehölzen mit unterschiedlich intensiver Nutzung auf ein und derselben Fläche wird heutzutage als ein Agroforstsystem (AFS) bezeichnet (Eichhorn et al., 2006; Herzog, 1997). Viele dieser historischen Nutzungssysteme sind, durch die Trennung von Landund Forstwirtschaft, verloren gegangen (Konold and Reeg, 2009).

Der Anbau von Paulownia in AFS wird in China jedoch schon seit Jahrhunderten praktiziert und spätestens seit den 1950er intensiviert und dabei auch wissenschaftlich begleitet (Wang and Shogren, 1992; Zhu et al., 1991, 1986). Seit den 1970er Jahren wird sie in den USA verstärkt auf Plantagen angepflanzt (Snow, 2015) und seit den 1990er Jahren wird sie auch in Neuseeland großflächig genutzt (Barton, 2007).

In Deutschland hat sich die Forschung erst seit den 2010er Jahren intensiviert (Bork et al., 2015; Felbermeier et al., 2015; Mosandl and Stimm, 2015; Stimm et al., 2013). So verwundert es also nicht, dass ihre mögliche Verwendung in Form von Agroforstsystemen noch nicht sehr weit bekannt, verbreitet und erforscht ist.

In traditioneller Weise werden die Baumarten der Gattung Paulownia in China zumeist in einer Mischkultur mit Nahrungspflanzen (Intercropping) angebaut. Dabei raten die Autoren Zhu et al. (1991, 1986) davon ab Paulownia mit anderen Lichtbaumarten zu mischen und stattdessen lieber unterständige und vor allem schattentolerante Arten, wie bspw. Bambus zu nutzen. 
Natürlich verringert sich mit dem Wachstum der Bäume das Licht, welches durch die Kronen fällt, was aber im Alter von $7-8$ Jahren ca. $40-50 \%$ der Freilandstrahlung entspricht. Selbst bei einem älteren, vollentwickelten Baum dringt noch immer zwischen $20-40 \%$ des Lichtes durch die Krone, was auch weiterhin den Anbau von einer großen Zahl verschiedener Feldfrüchte ermöglicht.

Dabei stehen die Anzahl der Bäume, also der Pflanzverband und der Ertrag an Feldfrüchten in direktem Zusammenhang mit dem Bewirtschaftungsziel. Dieses kann von primärer Holzgewinnung, über eine gleiche Aufteilung zwischen Holz- und Feldfrüchteproduktion, bis hin zu einem ausschließlichen Fokus auf den Feldfrüchteanbau variieren. (Zhu et al., 1991, 1986)

Dabei könnte die Baumart aufgrund ihrer Eigenschaften sowohl für die Wertholz- als auch für die Energieholzproduktion einzelstammweise verwendet werden (siehe hierzu Grünewald and Reeg, 2009).

Die in den Gewächshausexperimenten untersuchte Problematik der geringen Lichttoleranz kann, bei einem Anbau als Heister-Pflanze im Freiland mit genügend großen Pflanzabständen wie sie bei AFS der Fall sind, vernachlässigt werden.

Allerdings ist die Wasserverfügbarkeit während der Vegetationsperiode entscheidend zum Ausschöpfen des möglichen Wuchspotentials der Baumart. Im Bereich zwischen 60 bis $40 \%$ des bei den Gewächshausversuchen bereitgestellten Wassers, erhöhte sich die Ausfallrate zunehmend. Zhu et al. (1986) raten generell bei Trockenheit in der Vegetationsperiode zu einer aktiven Bewässerung, zumindest in den Anfangsjahren des Anbaus.

Um nicht nur ein Überleben, sondern nach Möglichkeit auch ein optimales Wachstum der Pflanzen sicherstellen zu können, sind neuartige Gestaltungsmethoden wie bspw. das Keyline-Design (Schlüssellinien) in AFS ratsam (Gerhardt, 2021). Hierbei wird die Tiefensickerung des Regenwasser gefördert, was speziell den Wurzeln der Paulownia zugutekommen würde, die in den ersten Jahren ihres Anwuchses ein möglichst tiefgehendes Wurzelsystem etabliert (Longbrake, 2001).

Die Wurzeltiefe ist entscheidend für die Konkurrenzstärke gegenüber den Feldfrüchten. Da die P.-Arten generell sehr tief wurzeln sind zumeist nur ca. $12 \%$ der Wurzeln in der für Feldfrüchte relevanten Bodentiefe von $0-40 \mathrm{~cm}$ zu finden. Mehr als $76 \%$ der Wurzelmasse eines Baumes findet sich hingegen in einer Tiefe von $40-100 \mathrm{~cm}$. Wie die Autoren Zhu et al. (1986) weiter am Beispiel eines adulten P.elongata-Baumes aufzeigen, verteilen sich ca. 70 - $85 \%$ der Absorptionswurzeln in einem Radius von 40 $-100 \mathrm{~cm}$ um den Baum herum. Eine solche natürliche Wurzelentwicklung ist besonders vorteilhaft in einer Mischung mit Feldfrüchten und prädestiniert die Paulownia für den Anbau in einem AFS. Dass sich die Baumart, neben der Nutzung als Einzelbaum in einem AFS, auch als Plantagenbaum auf Dauergrünland in Deutschland ökonomisch rechnet, hat Thielen (2019) nachgewiesen. 
Zhu et al. (1986) verweisen auf die verbesserten Wachstumsbedingungen für die Paulownia, die sich als Nebeneffekt durch die Düngung der Feldfrüchte ergeben. Außerdem heben sie die ca. $100 \mathrm{~kg}$ Blattmaterial (Frischmasse) hervor, die eine $8-10$ jährige Paulownia durchschnittlich produziert und die als nahrhaftes Viehfutter oder zusätzliche Düngung der Felder eingesetzt werden kann. Des Weiteren sind bei einem geplanten Umtrieb von 10 Jahren eine durchschnittliche Holzmasse von 0,4 bis $0,5 \mathrm{~m}^{3}$ Stammholz und zwischen 350 - $400 \mathrm{~kg}$ Astmaterial (evtl. Feuerholz) pro Baum zu erwarten, was die Wirtschaftlichkeit eines solchen Anbaus noch einmal betont. Es ist davon auszugehen, dass zukünftig auch der ökologische Aspekt, der schnellen $\mathrm{CO}^{2}-$ Bindung, noch zunehmend an Bedeutung gewinnen wird (Thielen, 2019; WeGrow, 2020).

Leider ist die Etablierung von AFS in Deutschland rechtlich noch nicht abschließend geklärt (Böhm et al., 2017; Chalmin and Möndel, 2009; Zehlius-Eckert, 2018) und die Gattung Paulownia bisher nicht förderfähig (VwG Köln, 2014).

Wie aber die Freilandversuche gezeigt haben, ist ein Anbau unter Beachtung der bereits ausführlich beschriebenen Anwuchsprobleme bei den Freilandversuchen, durchaus möglich und in Abhängigkeit von der ökonomischen Zielstellung des Flächenbesitzers (Möndel et al., 2009) auch sinnvoll. Zur Vermeidung von hohen Ausfallzahlen im Jugendstadium werden seitens der Paulownia-Saatgutfirmen zumeist stärker entwickelte, also ältere Wurzelstöcke bzw. Pflanzen ab drei Jahren empfohlen (Cathaia, 2020; EnPf, 2021).

\subsubsection{Anbauwürdigkeit vs. Invasivität}

Die Baumarten der Gattung Paulownia werden schon seit Jahrtausenden in China und über die Landesgrenzen hinaus aktiv von Menschen angebaut. Zwar dauerte es noch bis 1830 bis die erste Paulownia durch Philipp Franz von Siebold nach Europa eingeführt wurde, doch fand sie auch über andere eher indirekte Wege, bspw. in Form von Verpackungsmaterial (David, 2012), ihre frühe Verbreitung in die Welt.

Heutzutage werden Paulownia-Arten ganz selbstverständlich in örtlichen Baumärkten zum Verkauf angeboten und daher zeigen sich in jedem Frühjahr erneut ihre prachtvollen weiß-lila Blüten in den Vorgärten. Aus diesen verbreiten sie sich auf natürlichem Wege und völlig unkontrolliert in das nähere Umfeld, wie es Kiermeier schon 1977 beschrieben hat. Sehr häufig finden sich aber auch Einzelexemplare in der Nähe von bereits etablierten Plantagenbäumen, wie Essel (2007) erwähnt.

P.-Arten sind also mit einer gewissen Selbstverständlichkeit schon seit geraumer Zeit in der mitteleuropäischen Naturlandschaft zu finden. Zwar gibt es Anzeichen von Invasivität, wie sie bspw. in Form von Massenvermehrungen in den USA nach Waldbränden beschrieben werden (Innes, 2009; Remaley, 2005). Doch lassen sich diese Beobachtungen laut Nehring et al. (2013) nicht einfach auf mitteleuropäische Verhältnisse übertragen.

Nach der häufig verwendeten Definition von Kowarik (2010) handelt es sich bei einer Invasion um eine: „[...] Ausbreitung von Organismen in Gebieten, die sie auf natürliche Weise nicht erreicht haben.", wozu Vor et al. (2015) ganz richtig anmerken, dass dann ja auch alle einheimischen Baumarten als invasiv gelten müssten (siehe 2.1.4.2 Versuch einer forstlichen Invasivitätsbewertung). Eher anwendbar erscheint da die Definition nach Ammer et al. (2014) nach der eine Art als invasiv zu beurteilen ist, wenn von ihr eine reale und nachweisbare Gefahr ausgeht, die sich nicht steuern lässt. 
Um diese Gefahr der Invasivität bewerten zu können, beschreiben die Autoren Vor et al. (2015) fünf Kriterien. Sowohl die Fähigkeit zur Artenverdrängung als auch die negativen Beeinträchtigungen der Besiedelungs- und Lebensbedingungen für heimische Arten können für Paulownia als gegeben angesehen werden. Denn wie Innes (2009) erwähnt, ist die Paulownia durch ihr schnelles Höhenwachstum und das damit einhergehende große Blätterdach durchaus in der Lage die Regeneration einer natürlichen Waldgesellschaft auszudunkeln. Im Gegensatz dazu empfehlen Zhu et al. (1986) die Baumart ja gerade deswegen bevorzugt in AFS anzubauen, weil vergleichsweise viel Licht durch ihr vollausgebildetes Kronendach fällt, was den Feldfrüchten zu Gute kommt. Weitere wichtige Invasivitäts-Kriterien sind nach Vor et al. (2015) das hohe Reproduktions- und Ausbreitungspotential. Natürlich entstandene P.-Waldbestände in China sind aber sehr selten, da die Paulownia zumeist nur einzelstammweise an exponierten Standorten mit großem Lichtangebot, wie beispielsweise entlang von Flüssen oder offenen Tälern vorkommt (Zhu et al., 1986).

Es gibt folglich zahlreiche Bedenken und auf Grundlage der von Nehring et al. (2013) vorgenommen Einstufung als „potenziell invasive“ Baumart, ist eine Förderung im Rahmen von landwirtschaftlichen Dauerkulturen bisher nicht möglich (VwG Köln, 2014). Anders als es im Wald der Fall ist, wäre ein Anbau auf landwirtschaftlichen Flächen, allerdings ohne finanzielle Förderung bspw. seitens des Staates, auch weiterhin jederzeit möglich (EnPf, 2021; WeGrow, 2020).

Die Autoren Vor et al. (2015) betonen, dass bei einem möglichen Anbau in Form von landwirtschaftlichen Kurzumtriebsplantagen „[...] eine unerwünschte Ausbreitung in angrenzende Gebiete" unterbleibt, bzw. in der Nähe zu empfindlichen Biotopen ganz darauf verzichtet werden sollte (Döpke et al., 2013). In diesem Zusammenhang verwundert es folglich nicht, dass bspw. die Baumart P.tomentosa als nicht anbauwürdig für die deutschen Wälder eingestuft wird (Vor et al., 2015). Aller Voraussicht nach würden einzelne auf natürliche Weise verbreitende Exemplare allein schon durch die natürliche Sukzession der mitteleuropäischen Wälder verdrängt werden (Mosandl and Stimm, 2015).

$\mathrm{Ob}$ es allgemein zu einer Neubewertung hinsichtlich der potenziellen Invasivität (Nehring et al., 2013) kommt und Paulownia evtl. in gleicher Form wie die Art Robinia pseudoacacia bewertet wird (Vor et al., 2015), und sie folglich auch genauso als „bedingt anbauwürdig" eingestuft wird, bleibt jedoch weiterhin fraglich.

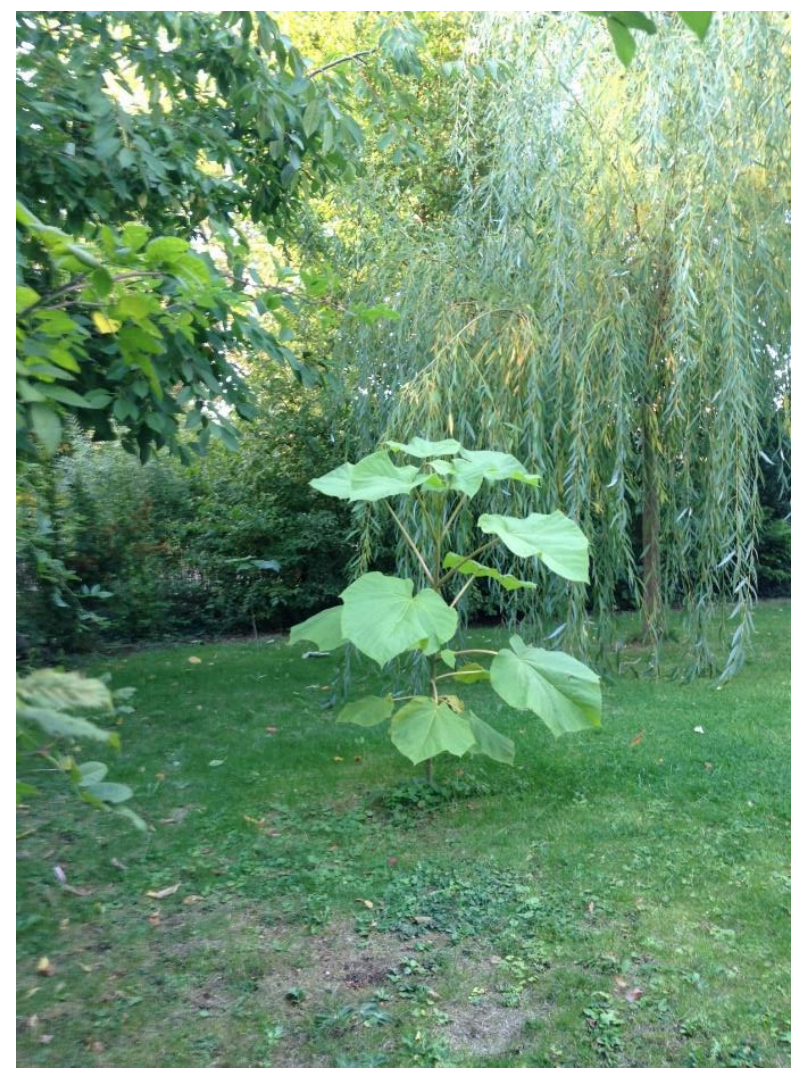

Abb. 47: Jahrestrieb einer P.elongata in einem privaten Vorgarten in der Leonard-Nelson-Str., in Göttingen. 


\section{Anhang zum Kapitel I}

Tab. 8: Bestimmungsschlüssel der Paulownia Arten im direkten Vergleich, verändert nach Zhu et al. (1986), S. 9.

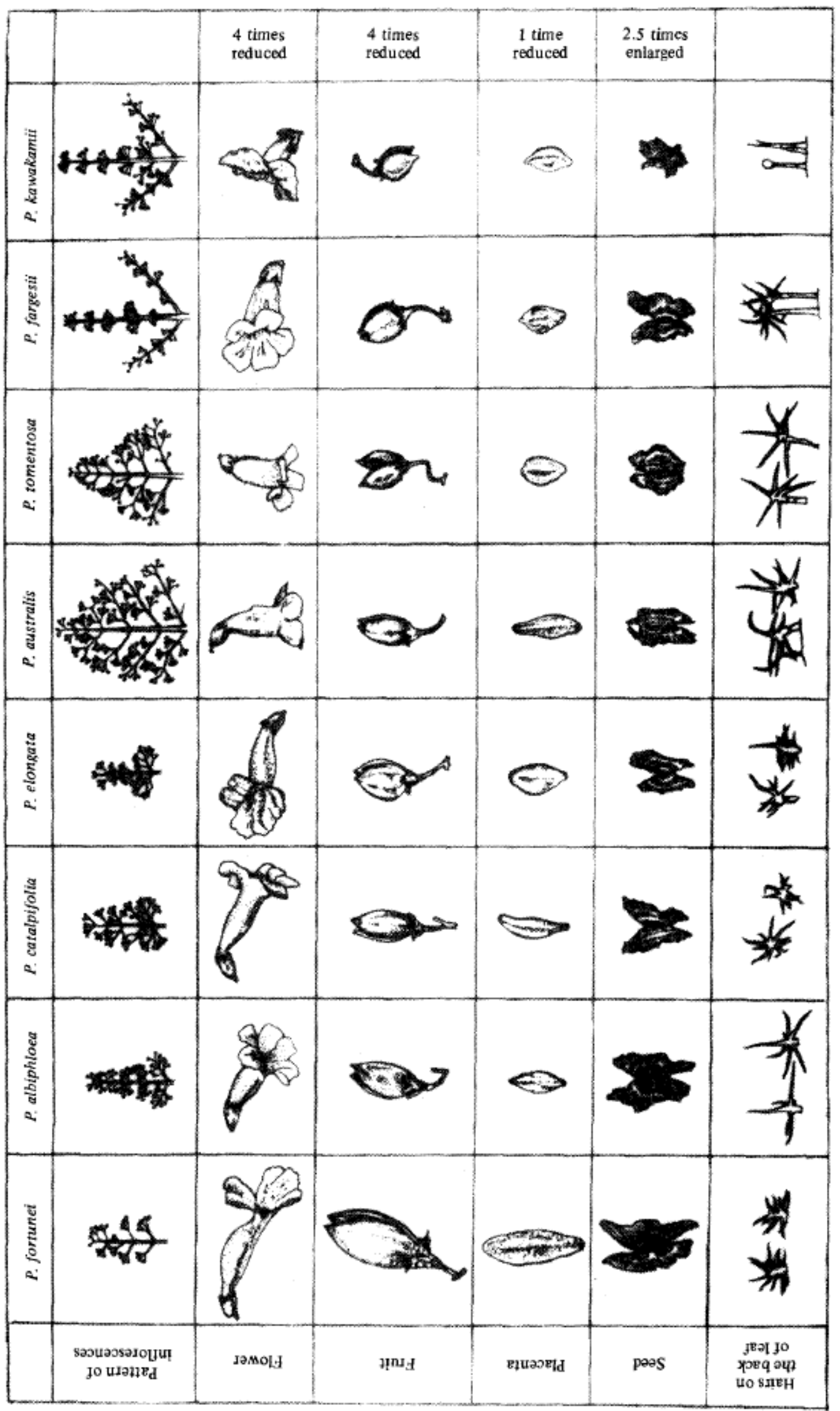


Tab. 9: Gesamtüberblick über die Arten und Hybriden die von Paulownia über International Plant Names Index (2020) bekannt sind:

Paulownia Siebold \& Zucc., Fl. Jap. (Siebold) 1: 25 (1835).

Paulownia australis T.Gong, Acta Phytotax. Sin. 14(2): 43 (1976).

Paulownia catalpifolia T.Gong, Acta Phytotax. Sin. 14(2): 41 (1976).

Paulownia catalpifolia T.Gong ex D.Y.Hong, Novon 7(4): 366 (1998).

Paulownia coreana Uyeki, Bull. Agric. Suigen, Corea i. 20, fig. 16 (1925); cf.

Hofker in Mitt.Deutsch. Dendrol. Ges. (1932), xliv. 417.

Paulownia duclouxii Dode, Bull. Soc. Dendrol. France (1908), 162.

Paulownia elongata S.Y.Hu, Quart. J. Taiwan Mus. xii. 41 (1959).

Paulownia fargesii Franch., Bull. Mus. Hist. Nat. Paris (1896), 280.

Paulownia fortunei (Seem.) Hemsl., J. Linn. Soc., Bot. 26: 180 (1890).

Paulownia glabrata Rehder, PI. Wilson. (Sargent) 1(3): 575 (1913).

Paulownia grandifolia hort. ex Wettst., Nat. Pflanzenfam. [Engler \& Prantl] iv. 3b (1891) 67 , in obs.

Paulownia $\times$ henanensis C.Y.Zhang \& Y.H.Zhao, Acta Phytotax. Sin. 33(5): 503 (1995).

Paulownia imperialis Siebold \& Zucc., Fl. Jap. (Siebold) 1: 25, t. 10 (1835), nom. illeg.

Paulownia $\times$ intermedia T.B.Lee, Bull. Kwanak Arbor. 4: 69 (1983), without latin descr. (1983).

Paulownia kawakamii T. Itô, Icon. PI. Japon. [Itô] 1(4): tt. 15-16 (1912).

Paulownia laotica Aver., PI. Diversity FI. Veg. Hin Nam No 116 (2019).

Paulownia lilacina Sprague, Bot. Mag. 147: t. 8926-7 (1938).

Paulownia longifolia Hand.-Mazz., Symb. Sin. Pt. VII. 832 (1936), in obs.; Hand.-

Mazz. in Beih. Bot. Centralbl. Ivi. B. 452 (1937).

Paulownia meridionalis Dode, Bull. Soc. Dendrol. France (1908), 162.

Paulownia mikado T. Itô, Icon. PI. Japon. [Itô] 1(3): tt. 9-12 (1911).

Paulownia recurva Rehder, PI. Wilson. (Sargent) 1(3): 577 (1913).

Paulownia rehderiana Hand.-Mazz., Anz. Akad. Wiss. Wien, Math.-Naturwiss. KI. (1921), Iviii. 153.

Paulownia shensiensis Pai, Contr. Inst. Bot. Natl. Acad. Peiping 3: 60 (1935).

Paulownia silvestrii Pamp. \& Bonati, Nuovo Giorn. Bot. Ital. xviii. 177 (1911).

Paulownia taiwaniana T.W.Hu \& H.J.Chang, Taiwania 20(2): 166 (1975).

Paulownia thyrsoidea Rehder, PI. Wilson. (Sargent) 1(3): 576 (1913).

Paulownia tomentosa (Thunb.) Steud., Nomencl. Bot. [Steudel], ed. 2. 2: 278 (1841).

Paulownia tomentosa Baill., Hist. PI. (Baillon) 9: 434 (1888).

Paulownia tomentosa var. glabrata (Rehder) S.Z.Qu, FI. Tsinling. 1(4): 319 (1983).

Paulownia tomentosa var. tsinlingensis (Pai) T.Gong, Acta Phytotax. Sin. 14(2): 43(1976).

Paulownia tomentosa f. virginea H.Ohashi, J. Jap. Bot. 92(5): 310 (2017).

Paulownia viscosa Hand.-Mazz., Sinensia 5: 7 (1934). 
Tab. 10: Tabelle über Pilze und Arthropoden die Paulownia nachweislich besiedeln. Die folgende Übersicht wurde übernommen und verändert nach Hao et al. (2004):

Legende:

\begin{tabular}{|l|l|}
\hline $\mathrm{m}=$ beschrieben auf P.tomentosa \\
\hline $\mathrm{mo}=$ beschrieben auf einer Art der Gattung Paulownia \\
\hline $\mathrm{O}=$ beschrieben auf mehr als einer Art der Gattung Paulownia, inklusive P.tomentosa \\
\hline $\mathrm{Oo}=$ beschrieben auf mehr als einer Art der Gattung Paulownia, ausgenommen \\
$\mathrm{P}$. tomentosa \\
\hline $\mathrm{p}=$ beschrieben auf Paulownia und anderen Gattungen \\
\hline $\begin{array}{l}\text { po }=\text { beschrieben auf mehr als einer Gattung, inklusive der Gattung Paulownia aber nicht } \\
\text { auf der Art P.tomentosa }\end{array}$ \\
\hline
\end{tabular}

Pilze:

\begin{tabular}{|c|c|c|c|}
\hline Phylum & Familie & Art & Quelle \\
\hline \multirow[t]{4}{*}{ Ascomycota } & \multirow[t]{3}{*}{ Erysiphaceae } & $\begin{array}{l}\text { Phyllactinia paulowniae } \\
\text { Yu }\end{array}$ & $\begin{array}{l}\text { m(Consilio Floraum, } \\
1987 \text { ) }\end{array}$ \\
\hline & & $\begin{array}{l}\text { Phyllactinia salmonii S. } \\
\text { Blumer }\end{array}$ & $\begin{array}{l}\text { oo(Consilio Floraum, } \\
1987 \text { ) }\end{array}$ \\
\hline & & Uncinula clintonii Peck & $\mathrm{p}$ (Dai, 1979) \\
\hline & Mycosphaerellaceae & $\begin{array}{l}\text { Mycosphaerella corylea } \\
\text { (Pers.) Karst. }\end{array}$ & m(Dai, 1979) \\
\hline Basidiomycota & Platygloeaceae & $\begin{array}{l}\text { Septobasidium tanakae } \\
\text { (Miyabe) Boedijn \& B.A. } \\
\text { Steinm. }\end{array}$ & $\mathrm{p}($ Dai, 1979) \\
\hline \multirow[t]{2}{*}{ Oomycota } & \multirow[t]{2}{*}{ Pythiaceae } & $\begin{array}{l}\text { Phytophthora palmivora } \\
\text { (E.J. Butler) E.J. Butler }\end{array}$ & po(Yun, 1998) \\
\hline & & $\begin{array}{l}\text { Pythium myrotylum } \\
\text { Drechsler }\end{array}$ & po(Yun, 1998) \\
\hline \multicolumn{2}{|c|}{ Anamorphic Diplocarpon } & $\begin{array}{l}\text { Gloeosporium kawakamii } \\
\text { Miyabe }\end{array}$ & m(Dai, 1979) \\
\hline \multirow{2}{*}{\multicolumn{2}{|c|}{ Anamorphic Mycosphaerella }} & $\begin{array}{l}\text { Cercospora paulowniae } \\
\text { Hori }\end{array}$ & $\begin{array}{l}\text { o(Dai, 1979), gilt als } \\
\text { Synonym von } \\
\text { Pseudocerospora } \\
\text { paulowniae Goh \& Hsieh }\end{array}$ \\
\hline & & $\begin{array}{l}\text { Pseudocercospora } \\
\text { paulowniae Goh \& W.H. } \\
\text { Hsieh }\end{array}$ & oo(Liu and Guo, 1998) \\
\hline \multicolumn{2}{|c|}{ Anamorphic Mycosphaerellaceae } & $\begin{array}{l}\text { Ascochyta paulowniae } \\
\text { Sacc. \& Brunaud }\end{array}$ & m(Dai, 1979) \\
\hline
\end{tabular}

Arthropoden:

\begin{tabular}{|l|l|l|l|}
\hline & Familie & Art & Quelle \\
\hline Coleoptera & Cerambycidae & Batocera horsfieldi (Hope) & $\begin{array}{l}\text { po(Chen et al., 1959), } \\
\text { po(Lei and Zhou, 1998), } \\
\text { po(Wu, 1995) }\end{array}$ \\
\cline { 3 - 4 } & & $\begin{array}{l}\text { Batocera lineolata } \\
\text { Chevrolat }\end{array}$ & po(Lei and Zhou, 1998) \\
\cline { 3 - 4 } & & & \\
\hline
\end{tabular}




\begin{tabular}{|c|c|c|}
\hline & Dere thoracica White & po(Lei and Zhou, 1998) \\
\hline & $\begin{array}{l}\text { Dorysthenes hydropicus } \\
\text { Pascoe }\end{array}$ & po(Lei and Zhou, 1998) \\
\hline & $\begin{array}{l}\text { Dorysthenes paradoxus } \\
\text { (Faldermann) }\end{array}$ & po(Lei and Zhou, 1998) \\
\hline & Megopis sinica White & po(Lei and Zhou, 1998) \\
\hline & Xylotrechus grayii (White) & $\begin{array}{l}\text { po(Chen et al., 1959), } \\
\text { po(Lei and Zhou, 1998) }\end{array}$ \\
\hline Cetoniidae & $\begin{array}{l}\text { Glycyphana horsfieldi } \\
\text { (Hope) }\end{array}$ & po(Huang, 1993) \\
\hline Chrysomelidae & $\begin{array}{l}\text { Aulacophora nigripennis } \\
\text { Motschulsky }\end{array}$ & po(Lei and Zhou, 1998) \\
\hline & $\begin{array}{l}\text { Gallerucida bifasciata } \\
\text { Motschulsky }\end{array}$ & mo(Lei and Zhou, 1998) \\
\hline & $\begin{array}{l}\text { Hemipyxis chinensis } \\
\text { (Weise) }\end{array}$ & mo(o.V., 1992a) \\
\hline & Hemipyxis jeanneli (Chen) & po(Yu et al., 1996) \\
\hline & $\begin{array}{l}\text { Hemipyxis plagioderoides } \\
\text { (Motschulsky) }\end{array}$ & $\begin{array}{l}\text { po(Huang, 1993), po(Lei } \\
\text { and Zhou, 1998), po(Yu } \\
\text { et al., 1996) }\end{array}$ \\
\hline & Oides bowringii (Baly) & po(Lei and Zhou, 1998) \\
\hline & Oides tarsatus (Baly) & po(Lei and Zhou, 1998) \\
\hline & $\begin{array}{l}\text { Paleosepharia fulvicornis } \\
\text { Chen }\end{array}$ & po(Lei and Zhou, 1998) \\
\hline & $\begin{array}{l}\text { Pseudespera paulowniae } \\
\text { Jiang }\end{array}$ & oo(o.V., 1992a) \\
\hline Crioceridae & $\begin{array}{l}\text { Sagra femorata purpurea } \\
\text { Lichtenstein }\end{array}$ & po(Lei and Zhou, 1998) \\
\hline & $\begin{array}{l}\text { Sagra fulgida janthina } \\
\text { Chen }\end{array}$ & po(Lei and Zhou, 1998) \\
\hline Curculionidae & $\begin{array}{l}\text { Phytoscaphus gossypii } \\
\text { Chao }\end{array}$ & po(Lei and Zhou, 1998) \\
\hline & $\begin{array}{l}\text { Piazomias fausti } \\
\text { Frivaldszky }\end{array}$ & mo(Lei and Zhou, 1998) \\
\hline & $\begin{array}{l}\text { Piazomias validus } \\
\text { Mostschulsky }\end{array}$ & $\begin{array}{l}\text { po(Chao and Chen, } \\
\text { 1980) }\end{array}$ \\
\hline & $\begin{array}{l}\text { Scythropus yasumatsui } \\
\text { Kono et Morimoto }\end{array}$ & $\begin{array}{l}\text { po(Chao and Chen, } \\
\text { 1980) }\end{array}$ \\
\hline Eumolpidae & Nodina tibialis Chen & $\begin{array}{l}\text { po(Lei and Zhou, 1998), } \\
\text { (Tang et al., 1980), } \\
\text { po(o.V., 1992a) }\end{array}$ \\
\hline Hispidae & $\begin{array}{l}\text { Basiprionota bisignata } \\
\text { (Boheman) }\end{array}$ & po(Wu, 1995) \\
\hline & $\begin{array}{l}\text { Basiprionota chinensis } \\
\text { (Fabricius) }\end{array}$ & $\begin{array}{l}\text { po(Huang, 1993), mo(Lei } \\
\text { and Zhou, 1998), } \\
\text { po(o.V., 1992a), po(Wu, } \\
\text { 1995) }\end{array}$ \\
\hline
\end{tabular}




\begin{tabular}{|c|c|c|c|}
\hline & & $\begin{array}{l}\text { Basiprionota whitei } \\
\text { (Boheman) }\end{array}$ & po(Lei and Zhou, 1998) \\
\hline & & $\begin{array}{l}\text { Laccoptera } \\
\text { quadrimaculata } \\
\text { (Thunberg) }\end{array}$ & po(Lei and Zhou, 1998) \\
\hline & Lucanidae & $\begin{array}{l}\text { Aegus laevicollis } \\
\text { Saunders }\end{array}$ & po(Huang, 1993) \\
\hline & & $\begin{array}{l}\text { Aegus parallelus Hope et } \\
\text { Westwood }\end{array}$ & po(Lei and Zhou, 1998) \\
\hline & Meloidae & Epicauta sibirica Pallas & po(Lei and Zhou, 1998) \\
\hline & Melolonthidae & $\begin{array}{l}\text { Holotrichia trichophora } \\
\text { (Fairmaire) }\end{array}$ & po(Lei and Zhou, 1998) \\
\hline & & $\begin{array}{l}\text { Maladera ovatula } \\
\text { (Fairmaire) }\end{array}$ & po(Lei and Zhou, 1998) \\
\hline & Rutelidae & $\begin{array}{l}\text { Anomala antiqua } \\
\text { (Gyllenhal) }\end{array}$ & po(Lei and Zhou, 1998) \\
\hline & Scolytidae & $\begin{array}{l}\text { Ambrosiodmus rubricollis } \\
\text { (Eichhoff) }\end{array}$ & po(Huang, 1993) \\
\hline Hemiptera & Berytidae & $\begin{array}{l}\text { Gampsocors pulchellus } \\
\text { (Dallas) }\end{array}$ & $\begin{array}{l}\text { mo(Lei and Zhou, 1998), } \\
\text { po(Zhang, 1985) }\end{array}$ \\
\hline & & Yemma signatus (Hsiao) & mo(Lei and Zhou, 1998) \\
\hline & Coreidae & Cletus tenuis Kiritshenko & po(Lei and Zhou, 1998) \\
\hline & Miridae & $\begin{array}{l}\text { Gallobelicus crassicornis } \\
\text { Distand }\end{array}$ & po(Zhang, 1985) \\
\hline & & $\begin{array}{l}\text { Nesidiocoris tenuis } \\
\text { (Reuter) }\end{array}$ & $\begin{array}{l}\text { mo(Lei and Zhou, 1998), } \\
\text { beschrieben als } \\
\text { Cyrtopeltis tenius Reuter }\end{array}$ \\
\hline & Pentatomidae & Dalpada cinctipes Walker & po(Huang, 1993) \\
\hline & & $\begin{array}{l}\text { Dolycoris baccarum } \\
\text { (Linnaeus) }\end{array}$ & $\begin{array}{l}\text { po(Huang, 1993), } \\
\text { po(Zhang, 1985) }\end{array}$ \\
\hline & & Erthesina fullo (Thunberg) & $\begin{array}{l}\text { po(Lei and Zhou, 1998), } \\
\text { po(Zhang, 1985) }\end{array}$ \\
\hline & & Eurostus grossipe Dallas & po(Huang, 1993) \\
\hline & & Eurostus validus Dallas & po(Lei and Zhou, 1998) \\
\hline & & $\begin{array}{l}\text { Lamprocoris roylii } \\
\text { (Westwood) }\end{array}$ & mo(Huang, 1993) \\
\hline & & Laprius varicornis (Dallas) & $\begin{array}{l}\text { po(Huang, 1993), po(Lei } \\
\text { and Zhou, 1998) }\end{array}$ \\
\hline & & $\begin{array}{l}\text { Menida metalliea Hsiao et } \\
\text { Cheng }\end{array}$ & m(Zhang, 1995) \\
\hline & & Menida scotti Puton & po(Zhang, 1995) \\
\hline & & $\begin{array}{l}\text { Plautia fimbriata } \\
\text { (Fabricius) }\end{array}$ & $\begin{array}{l}\text { po(Lei and Zhou, 1998), } \\
\text { po(Zhang, 1985) }\end{array}$ \\
\hline & & Rubiconia peltata Jakovlev & mo(Lei and Zhou, 1998) \\
\hline & & Stollia guttiger (Thunberg) & $\begin{array}{l}\text { po(Lei and Zhou, 1998), } \\
\text { po (Zhang, 1985) }\end{array}$ \\
\hline & Tingidae & $\begin{array}{l}\text { Eteoneus angulatus Drake } \\
\text { et Maa }\end{array}$ & $\begin{array}{l}\text { mo(Lei and Zhou, 1998), } \\
\text { m(Zhang, 1985) }\end{array}$ \\
\hline
\end{tabular}




\begin{tabular}{|c|c|c|c|}
\hline \multirow[t]{10}{*}{ Homoptera } & \multirow[t]{4}{*}{ Cicadellidae } & $\begin{array}{l}\text { Empoasca flavescens } \\
\text { (Fabricius) }\end{array}$ & po(Lei and Zhou, 1998) \\
\hline & & $\begin{array}{l}\text { Hishimonus sellatus } \\
\text { (Uhler) }\end{array}$ & po(Shen and Shi, 1998) \\
\hline & & $\begin{array}{l}\text { Tettigoniella ferruginea } \\
\text { (Fabricius) }\end{array}$ & $\begin{array}{l}\text { po(Lei and Zhou, 1998), } \\
\text { beschrieben als } \\
\text { Tettigella ferruginea } \\
\text { (Fabricius) }\end{array}$ \\
\hline & & Tettigoniella viridis (Linné) & $\begin{array}{l}\text { po(Lei and Zhou, 1998), } \\
\text { beschrieben als } \\
\text { Cicadella viridis } \\
\text { Linnaeus }\end{array}$ \\
\hline & \multirow[t]{2}{*}{ Cicadidae } & $\begin{array}{l}\text { Oncotympana } \\
\text { maculaticollis } \\
\text { (Motschulsky) }\end{array}$ & $\begin{array}{l}\text { po(Lei and Zhou, 1998), } \\
\text { po(Wu, 1995) }\end{array}$ \\
\hline & & $\begin{array}{l}\text { Platypleura kaempferi } \\
\text { (Fabricius) }\end{array}$ & po(Lei and Zhou, 1998) \\
\hline & Diaspididae & $\begin{array}{l}\text { Pseudaulacaspis } \\
\text { pentagona (Targioni- } \\
\text { Tozzetti) }\end{array}$ & $\begin{array}{l}\text { po(Lei and Zhou, 1998), } \\
\text { po(Wu, 1995) }\end{array}$ \\
\hline & Margarodidae & $\begin{array}{l}\text { Drosicha corpulenta } \\
\text { (Kuwana) }\end{array}$ & po(Lei and Zhou, 1998) \\
\hline & Membracidae & $\begin{array}{l}\text { Jingkara hyalipunctata } \\
\text { Chou }\end{array}$ & po(Lei and Zhou, 1998) \\
\hline & Ricaniidae & Ricania sublimbata Jacobi & po(Lei and Zhou, 1998) \\
\hline Isoptera & Termitidae & Macrotermes barneyi Light & po(Lei and Zhou, 1998) \\
\hline \multirow[t]{10}{*}{ Lepidoptera } & \multirow[t]{3}{*}{ Arctiidae } & Hyphantria cunea (Drury) & po(Fang, 2000) \\
\hline & & Lemyra melli (Daniel) & $\begin{array}{l}\text { po(Fang, 2000), po(o.V., } \\
\text { 1992b), dabei } \\
\text { beschrieben als } \\
\text { Spilarctia melli Daniel }\end{array}$ \\
\hline & & $\begin{array}{l}\text { Lemyra proteus (DE } \\
\text { Joannis) }\end{array}$ & po(Fang, 2000) \\
\hline & Eupterotidae & Eupterote chinensis Leech & po(Lei and Zhou, 1998) \\
\hline & \multirow[t]{4}{*}{ Geometridae } & $\begin{array}{l}\text { Ascotis selenaria dianaria } \\
\text { Hübner }\end{array}$ & po(Lei and Zhou, 1998) \\
\hline & & $\begin{array}{l}\text { Biston marginata } \\
\text { Matsumura }\end{array}$ & po(Lei and Zhou, 1998) \\
\hline & & $\begin{array}{l}\text { Culcula panterinaria } \\
\text { (Bremer et Grey) }\end{array}$ & $\begin{array}{l}\text { po(Huang, 1993), po(Lei } \\
\text { and Zhou, 1998), po(Wu, } \\
\text { 1995) }\end{array}$ \\
\hline & & Odontopera aurata (Prout) & po(Wu, 1995) \\
\hline & \multirow[t]{2}{*}{ Hepialidae } & $\begin{array}{l}\text { Phassus excrescens } \\
\text { Butler }\end{array}$ & po(Lei and Zhou, 1998) \\
\hline & & $\begin{array}{l}\text { Phassus sinifer sinensis } \\
\text { Moore }\end{array}$ & po(Lei and Zhou, 1998) \\
\hline
\end{tabular}




\begin{tabular}{|c|c|c|}
\hline \multirow[t]{3}{*}{ Limacodidae } & $\begin{array}{l}\text { Latoia hilarata } \\
\text { (Staudinger) }\end{array}$ & $\begin{array}{l}\text { po(Lei and Zhou, 1998), } \\
\text { beschrieben als Parasa } \\
\text { hilarata (Staudinger) }\end{array}$ \\
\hline & Parasa consocia Walker & po(Lei and Zhou, 1998) \\
\hline & Thosea sinensis (Walker) & $\begin{array}{l}\text { po(Huang, 1993), po(Lei } \\
\text { and Zhou, 1998), po(Wu, } \\
\text { 1995) }\end{array}$ \\
\hline \multirow[t]{8}{*}{ Lymantriidae } & Dasychira grotei Moore & $\begin{array}{l}\text { po(Huang, 1993), mo(Lei } \\
\text { and Zhou, 1998) }\end{array}$ \\
\hline & $\begin{array}{l}\text { Dasychira horsfieldi } \\
\text { Saunders }\end{array}$ & po(Zhao, 1994) \\
\hline & $\begin{array}{l}\text { Euproctis bipunctapex } \\
\text { (Hampson) }\end{array}$ & po(o.V., 1992b) \\
\hline & $\begin{array}{l}\text { Euproctis } \\
\text { pseudoconspersa Strand }\end{array}$ & po(Lei and Zhou, 1998) \\
\hline & Lymantria mathura Moore & $\begin{array}{l}\text { po(o.V., 1992b), po(Wu, } \\
\text { 1995) }\end{array}$ \\
\hline & $\begin{array}{l}\text { Porthesia atereta } \\
\text { Collenette }\end{array}$ & $\begin{array}{l}\text { po(Huang, 1993), po(Lei } \\
\text { and Zhou, 1998), po(Wu, } \\
\text { 1995) }\end{array}$ \\
\hline & $\begin{array}{l}\text { Porthesia scintillans } \\
\text { (Walker) }\end{array}$ & $\begin{array}{l}\text { po(Huang, 1993), po(Lei } \\
\text { and Zhou, 1998) }\end{array}$ \\
\hline & Porthesia similis (Fueszly) & $\begin{array}{l}\text { po(Huang, 1993), po(Lei } \\
\text { and Zhou, 1998), po(Wu, } \\
\text { 1995) }\end{array}$ \\
\hline \multirow[t]{6}{*}{ Noctuidae } & $\begin{array}{l}\text { Argyrogramma agnata } \\
\text { Staudinger }\end{array}$ & $\begin{array}{l}\text { po(Lei and Zhou, 1998), } \\
\text { beschrieben als Plusia } \\
\text { agnata (Staudinger) }\end{array}$ \\
\hline & Artena dotata (Fabricius) & $\begin{array}{l}\text { po(Lei and Zhou, 1998), } \\
\text { beschrieben als } \\
\text { Laceoptera dotata } \\
\text { Fabricius }\end{array}$ \\
\hline & Euxoa oberthuri Leech & po(Lei and Zhou, 1998) \\
\hline & $\begin{array}{l}\text { Helicoverpa armigera } \\
\text { (Hübner) }\end{array}$ & po(Lei and Zhou, 1998) \\
\hline & Prodenia litura (Fabricius) & po(Lei and Zhou, 1998) \\
\hline & $\begin{array}{l}\text { Spodoptera exigua } \\
\text { (Hübner) }\end{array}$ & $\begin{array}{l}\text { po(Lei and Zhou, 1998), } \\
\text { beschrieben als } \\
\text { Laohygama exigua } \\
\text { Hübner }\end{array}$ \\
\hline Nymphalidae & Junonia orithya Linnaeus & po(Wu, 1995) \\
\hline Psychidae & Chalia larminati Heylaerts & po(o.V., 1992b) \\
\hline
\end{tabular}




\begin{tabular}{|c|c|c|c|}
\hline & & Ctania variegata Snellen & $\begin{array}{l}\text { po(Lei and Zhou, 1998), } \\
\text { beschrieben als } \\
\text { Cryptohelea variegata } \\
\text { Snellen po (o.V., 1992b), } \\
\text { erfasst als Eumeta } \\
\text { variegata Snellen, } \\
\text { po(Wu, 1995) }\end{array}$ \\
\hline & Pyralidae & $\begin{array}{l}\text { Dichocrocis chlorophanta } \\
\text { Butler }\end{array}$ & po(Lei and Zhou, 1998) \\
\hline & & $\begin{array}{l}\text { Mimicia pseudolibatrix } \\
\text { Caradja }\end{array}$ & m(Huang, 1993) \\
\hline & & $\begin{array}{l}\text { Pycnarmon cribrata } \\
\text { (Fabricus) }\end{array}$ & $\begin{array}{l}\text { m(Huang, 1993), po(Lei } \\
\text { and Zhou, 1998), m(o.V., } \\
\text { 1992b), mo(Wang, 1980) }\end{array}$ \\
\hline & Saturniidae & Attacus atlas (Linnaeus) & $\begin{array}{l}\text { po(Huang, 1993), } \\
\text { po(Zhu, 1996) }\end{array}$ \\
\hline & & $\begin{array}{l}\text { Eriogyna pyretorum } \\
\text { cognata Jordan }\end{array}$ & po(Zhu, 1996) \\
\hline & & $\begin{array}{l}\text { Eriogyna pyretorum } \\
\text { pyretorum Westwood }\end{array}$ & po(Zhu, 1996) \\
\hline & & Samia cynthia (Drurvy) & $\begin{array}{l}\text { po(Lei and Zhou, 1998), } \\
\text { beschrieben als } \\
\text { Philosamia cynthia } \\
\text { Walker et Felder }\end{array}$ \\
\hline & & $\begin{array}{l}\text { Samia cynthia insularis } \\
\text { (Vollendofen) }\end{array}$ & po(Zhu, 1996) \\
\hline & Sphingidae & $\begin{array}{l}\text { Clanis bilineata tsingtauica } \\
\text { mell }\end{array}$ & po(Lei and Zhou, 1998) \\
\hline & & Dolbina tancrei Staudinger & po(Lei and Zhou, 1998) \\
\hline & & Parum colligata (Walker) & po(Lei and Zhou, 1998) \\
\hline & & $\begin{array}{l}\text { Psilogramma increta } \\
\text { (Walker) }\end{array}$ & po(Lei and Zhou, 1998) \\
\hline & & $\begin{array}{l}\text { Psilogramma menephron } \\
\text { (Cramer) }\end{array}$ & $\begin{array}{l}\text { po(Lei and Zhou, 1998), } \\
\text { po(o.V., 1992b), po(Zhu, } \\
\text { 1980), po(Zhu and } \\
\text { Wang, 1997) }\end{array}$ \\
\hline & & $\begin{array}{l}\text { Theretra oldenlandiae } \\
\text { (Fabricius) }\end{array}$ & po(Lei and Zhou, 1998) \\
\hline & Tortricidae & $\begin{array}{l}\text { Homona coffearia } \\
\text { (Nietner) }\end{array}$ & po(Lei and Zhou, 1998) \\
\hline Orthoptera & Pyrgomorphidae & $\begin{array}{l}\text { Atractomorpha sinensis I. } \\
\text { Bolivar }\end{array}$ & po(Lei and Zhou, 1998) \\
\hline Thysanoptera & Thripidae & $\begin{array}{l}\text { Selenothrips rubrocinctus } \\
\text { (Giard) }\end{array}$ & po(Huang, 1993) \\
\hline & & Thrips andrewsi (Bagnall) & mo(Lei and Zhou, 1998) \\
\hline
\end{tabular}


Tab. 12: Tabellarische Auflistung der Holzeigenschaften von verschiedenen PaulowniaArten, übernommen und verändert nach Zhu et al. (1986), S. 59.

\begin{tabular}{|c|c|c|c|c|c|c|c|c|c|c|c|c|c|c|c|c|c|c|c|c|c|c|}
\hline \multirow[t]{3}{*}{ Species } & \multirow[t]{3}{*}{$\begin{array}{l}\text { Location } \\
\text { (Lat.) }\end{array}$} & \multirow{3}{*}{ 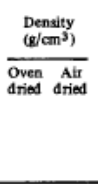 } & \multicolumn{3}{|c|}{$\begin{array}{c}\text { Shrinkage } \\
\text { coefmicient } \\
(6)\end{array}$} & \multirow{3}{*}{$\begin{array}{c}\text { Compression } \\
\text { strength } \\
\text { paraliel } \\
\text { to grain } \\
\left(\mathbf{k g f} / \mathrm{cm}^{2}\right)\end{array}$} & \multirow{3}{*}{$\begin{array}{l}\text { Bending } \\
\text { sterength } \\
\left(\mathrm{kgg}^{\left.\mathrm{g} f \mathrm{~cm}^{2}\right)}\right.\end{array}$} & \multirow{3}{*}{$\begin{array}{c}\text { Bending } \\
\text { modulus } \\
\text { of elasticity } \\
(1,000 \\
\left.\mathrm{kgt}^{2} / \mathrm{cm}^{2}\right)\end{array}$} & \multicolumn{2}{|c|}{$\begin{array}{c}\text { Shear strength } \\
\text { parallel to grath } \\
\left(\mathrm{kgf} / \mathrm{/m} \mathrm{m}^{2}\right) \\
\end{array}$} & \multicolumn{4}{|c|}{$\begin{array}{l}\text { Compression strength } \\
\text { perpendiculast to grain } \\
\left(\mathrm{kg}^{2} / \mathrm{cm}^{2}\right)\end{array}$} & \multirow{3}{*}{$\begin{array}{c}\text { Tensile } \\
\text { strength } \\
\text { parallel } \\
\text { to grain } \\
\left(\mathrm{kgg}^{\prime} f\left(\mathrm{~cm}^{2}\right)\right.\end{array}$} & \multirow{3}{*}{ 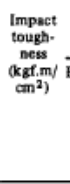 } & \multicolumn{3}{|c|}{$\begin{array}{l}\text { Hardness } \\
\left(\mathrm{kgE}_{\mathrm{E}} f \mathrm{~cm}^{2}\right)\end{array}$} & \multicolumn{2}{|c|}{$\begin{array}{c}\text { Cleavage } \\
\text { resistance } \\
\left(\mathrm{kgg}^{\prime} f\left(\mathrm{~cm}^{2}\right)\right.\end{array}$} & \multirow[t]{3}{*}{$\begin{array}{l}\text { Quality } \\
\text { coefficient }\end{array}$} \\
\hline & & & Radial & $\begin{array}{l}\text { Tang: } \\
\text { gential }\end{array}$ & Volume & & & & $\begin{array}{l}\text { Radial } \\
\text { section }\end{array}$ & $\begin{array}{c}\text { Tangential } \\
\text { section }\end{array}$ & $\begin{array}{c}\text { Para } \\
\text { commpe } \\
\text { propo } \\
\text { Int }\end{array}$ & $\begin{array}{l}\text { trial } \\
\text { tasion at } \\
\text { rtional } \\
\text { nit }\end{array}$ & $\begin{array}{c}\text { Compres } \\
\text { propor } \\
\text { lim }\end{array}$ & $\begin{array}{l}\text { esssion at } \\
\text { entrional } \\
\text { mate }\end{array}$ & & & End & $\begin{array}{l}\text { Radial } \\
\text { section }\end{array}$ & $\begin{array}{l}\text { Tan. } \\
\text { gential } \\
\text { Section }\end{array}$ & Radial & $\begin{array}{c}\text { Tan- } \\
\text { gential } \\
\text { section }\end{array}$ & \\
\hline & & & & & & & & & & & Radial & 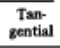 & Radial & $\underset{\substack{\text { Tan- } \\
\text { gential }}}{\text {. }}$ & & & & & & & & \\
\hline P. catalpiffolia & $\begin{array}{l}\text { Sueng County, } \\
\text { Henan Province } \\
\left(34^{\circ} S 2^{\prime}\right)\end{array}$ & 0.2330 .290 & 0.093 & 0.216 & 0.344 & 196 & 329 & 54 & 41 & 47 & 28 & 20 & 17 & 11 & 521 & 0.171 & 151 & 87 & 94 & 7.7 & 8.3 & 1,810 \\
\hline \multirow[t]{2}{*}{ P. elongata } & \multirow{2}{*}{$\begin{array}{l}\text { Fugou County, } \\
\text { Henan Provingoe } \\
\text { Lankau County, } \\
\text { Henan Provinceo } \\
\left(34^{\circ} 2 \sigma^{\prime}\right)\end{array}$} & 0.2090 .264 & 0.076 & 0.187 & 0.292 & 159 & 289 & 42 & 44 & 44 & 22 & 16 & 14 & 12 & 394 & 0.132 & 125 & 84 & 86 & 7.6 & 6.3 & 1,697 \\
\hline & & 0.2430 .283 & 0.147 & 0.269 & 0.453 & 197 & 356 & 44 & 40 & 39 & 24 & 22 & 16 & 12 & - & 0.180 & 195 & 99 & 122 & 6.5 & 6.3 & 1,954 \\
\hline P. farbesii & $\begin{array}{l}\text { Muchuan County, } \\
\text { Szzechuan Province } \\
\left(29^{\circ} 00^{\prime}\right)\end{array}$ & 0.2190 .269 & 0.107 & 0.216 & 0.334 & 160 & 363 & $\$ 2$ & 42 & 35 & 21 & 24 & 14 & 18 & 518 & 0.214 & 171 & 114 & 121 & 7.6 & 6.1 & 1.944 \\
\hline P. fortunel & $\begin{array}{l}\text { Gultu County, } \\
\text { Szzechuan Province } \\
\text { (28 }\end{array}$ & 0.2580 .309 & 0.110 & 0.210 & 0.320 & 188 & 405 & 63 & 56 & so & 29 & 27 & 21 & 19 & 563 & 0.325 & 215 & 124 & 124 & 7.6 & 7.4 & 1,919 \\
\hline \multirow[t]{2}{*}{ P. tomentosa } & \multirow{2}{*}{$\begin{array}{l}\text { Fugou County, } \\
\text { Henan Province } \\
\text { Shu County. } \\
\text { Anhei Province } \\
\left(34^{\circ} 20^{\prime}\right)\end{array}$} & 0.2360 .315 & 0.105 & 0.203 & 0.327 & 223 & 406 & 48 & 51 & 56 & 35 & 28 & 20 & 20 & 605 & 0.348 & 183 & 117 & 135 & 10.9 & 9.6 & 1,997 \\
\hline & & 0.2310 .278 & 0.079 & 0.164 & 0.261 & 200 & 381 & 50 & 47 & 45 & 23 & 22 & 16 & 13 & 343 & 0.240 & 189 & 98 & 106 & 7.0 & 6.0 & 2,090 \\
\hline $\begin{array}{l}\text { P. tomentosa } \\
\text { var. } \\
\text { tsintingensis }\end{array}$ & $\begin{array}{l}\text { Fugou County, } \\
\text { Henan Province } \\
\left(34^{\circ} 20^{\prime}\right)\end{array}$ & 0.2790 .347 & 0.107 & 0.208 & 0.333 & 220 & 415 & 58 & 59 & 54 & 30 & 30 & 17 & 20 & 568 & 0.416 & 198 & 142 & 143 & 10.2 & 9.8 & 1,830 \\
\hline
\end{tabular}

Tab. 11: Bewertung der Invasivität von Baumarten aus Sicht der Forstwissenschaften, entnommen und verändert nach Vor et al. (2015).

\begin{tabular}{|c|c|c|c|c|c|c|c|c|}
\hline \multirow[b]{2}{*}{ Baumarten } & \multicolumn{5}{|c|}{ Invasivitätskriterien } & \multicolumn{2}{|c|}{$\begin{array}{l}\text { Gesamt- } \\
\text { bewertung }\end{array}$} & \multirow[b]{2}{*}{$\begin{array}{l}\text { Ausschlussgründe } \\
\text { für } \\
\text { Anbauwürdigkeit }\end{array}$} \\
\hline & 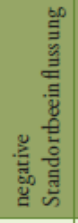 & 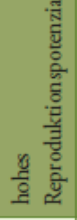 & 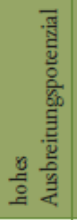 & 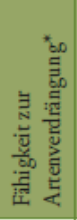 & 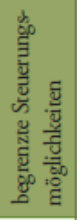 & 新 & 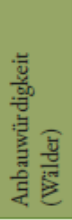 & \\
\hline $\begin{array}{l}\text { Abies grandis } \\
\text { Große Küstentanne }\end{array}$ & O & 1 & O & 0 & 0 & nein & ja & - \\
\hline $\begin{array}{l}\text { Acer negundo } \\
\text { Eschenahorn }\end{array}$ & 0 & ○ & ○ & ○ & ○ & ja & nein & invasiv in Auwäldern \\
\hline $\begin{array}{l}\text { Ailanthus altissima } \\
\text { Götterbaum }\end{array}$ & 1 & - & - & - & - & ja & nein & $\begin{array}{l}\text { konkurrenzschwach in Wäl- } \\
\text { dern, invasiv im Offenland }\end{array}$ \\
\hline $\begin{array}{l}\text { Fraxinus pennsylvanica } \\
\text { Rotesche }\end{array}$ & 0 & ○ & ○ & 1 & - & ja & nein & invasiv in Auwäldern \\
\hline $\begin{array}{l}\text { Gleditsia triacanthos } \\
\text { Gleditschie }\end{array}$ & 1 & - & 1 & 1 & 1 & $\begin{array}{l}\text { be- } \\
\text { dingt }\end{array}$ & nein & $\begin{array}{l}\text { konkurrenzschwach in Wäl- } \\
\text { dern, invasiv im Offenland }\end{array}$ \\
\hline $\begin{array}{l}\text { Larix kaempferi } \\
\text { Japanlärche }\end{array}$ & 1 & 1 & 0 & 0 & 0 & nein & ja & - \\
\hline $\begin{array}{l}\text { Paulownia tomentosa } \\
\text { Paulownie }\end{array}$ & 1 & - & 1 & 1 & 1 & $\begin{array}{l}\text { be- } \\
\text { dingt }\end{array}$ & nein & $\begin{array}{l}\text { konkurrenzschwach in Wäl- } \\
\text { dern, invasiv im Offenland }\end{array}$ \\
\hline $\begin{array}{l}\text { Pinus nigra } \\
\text { Schwarzkiefer }\end{array}$ & 1 & 0 & 0 & 0 & 0 & nein & ja & - \\
\hline $\begin{array}{l}\text { Pinus strobus } \\
\text { Strobe }\end{array}$ & O & ○ & 1 & O & 0 & nein & nein & $\begin{array}{l}\text { Schädigung durch } \\
\text { Strobenrost }\end{array}$ \\
\hline $\begin{array}{l}\text { Populus } x \text { canadensis } \\
\text { Hybridpappel }\end{array}$ & O & - & O & 1 & 0 & $\begin{array}{l}\text { be- } \\
\text { dingt }\end{array}$ & $\begin{array}{l}\text { be- } \\
\text { dingt }\end{array}$ & $\begin{array}{l}\text { Introgression } \\
\text { Schwarzpappel }\end{array}$ \\
\hline $\begin{array}{l}\text { Prunus serotina } \\
\text { Spät. Traubenkirsche }\end{array}$ & 0 & - & - & - & - & ja & nein & $\begin{array}{l}\text { invasiv in lichten Eichen- } \\
\text { und Kiefernwäldern }\end{array}$ \\
\hline $\begin{array}{l}\text { Pseudotsuga menziesii } \\
\text { Douglasie }\end{array}$ & 0 & 0 & ○ & 0 & 0 & nein & ja & - \\
\hline $\begin{array}{l}\text { Quercus rubra } \\
\text { Roteiche }\end{array}$ & 0 & 1 & 0 & 0 & 0 & nein & ja & - \\
\hline $\begin{array}{l}\text { Rhus typhina } \\
\text { Essigbaum }\end{array}$ & O & - & 1 & 1 & 1 & $\begin{array}{l}\text { be- } \\
\text { dingt }\end{array}$ & nein & $\begin{array}{l}\text { konkurrenzschwach in Wäl- } \\
\text { dern, invasiv im Offenland }\end{array}$ \\
\hline $\begin{array}{l}\text { Robinia psendoacacia } \\
\text { Robinie }\end{array}$ & 1 & - & 1 & 1 & 1 & $\begin{array}{l}\text { be- } \\
\text { dingt }\end{array}$ & $\begin{array}{l}\text { be- } \\
\text { dingt }\end{array}$ & $\begin{array}{l}\text { invasiv im Offenland, Stand- } \\
\text { orteinfluss (N-Fixierung) }\end{array}$ \\
\hline
\end{tabular}

* ausgenommen sind Tendenzen auf Sonderstandorten

Symbole:

trifft nicht $\mathrm{zu}$

1 trifft bedingt zu

- trifft zu 


\section{Anhang zum Kapitel II}

Abb. 48: Naturschutzfachliche Invasivitätsbewertung von P.tomentosa, entnommen und verändert nach Nehring et al. (2013).

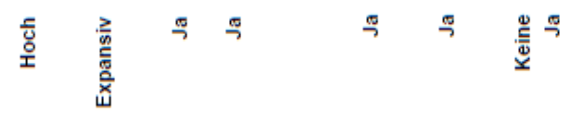
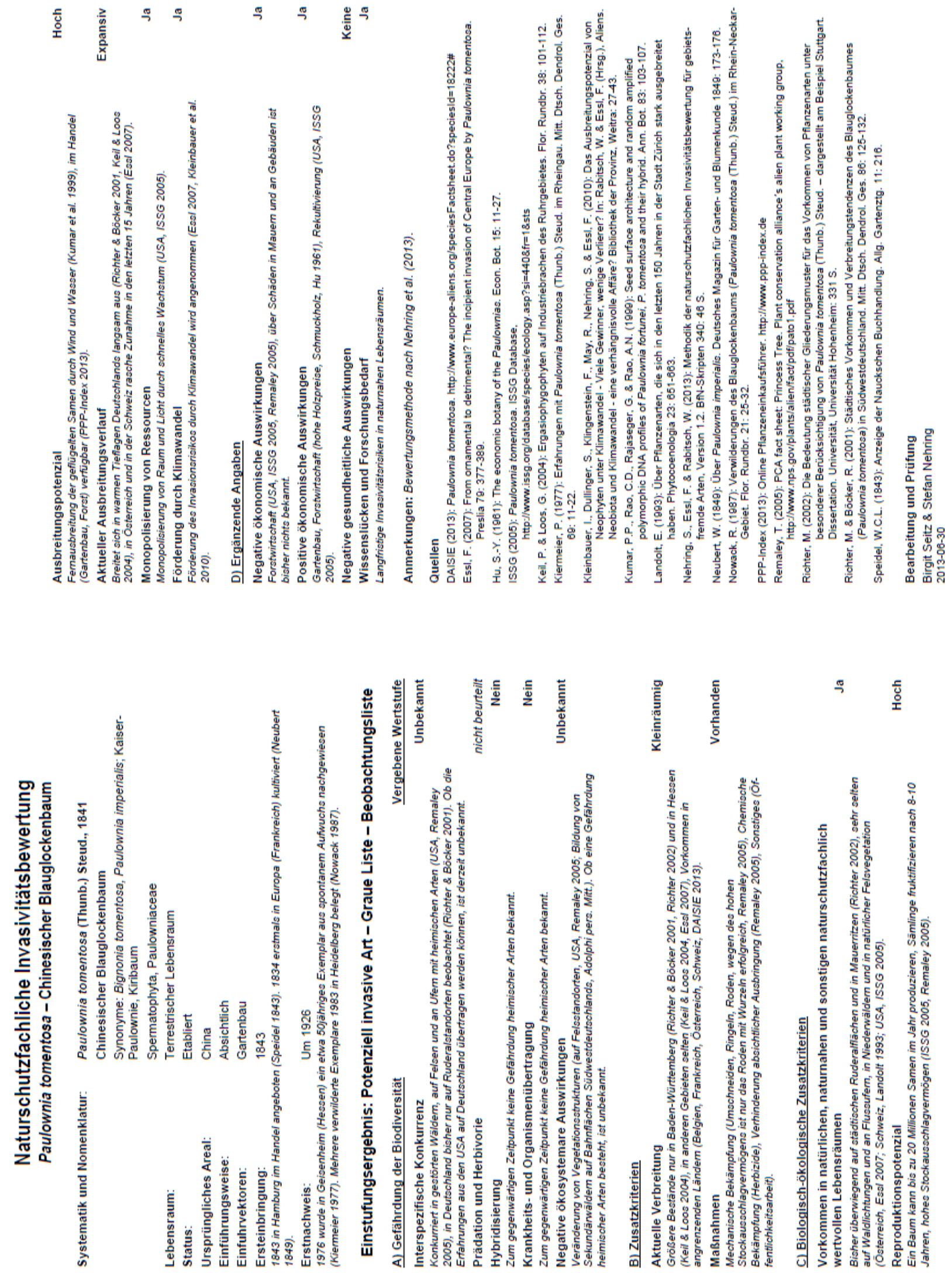

$\stackrel{5}{\circ} \quad \frac{5}{x}$ 


\section{Anhang zum Kapitel III}

Abb. 49: Informationsblätter zum Bodenhilfsstoff: Geohumus.
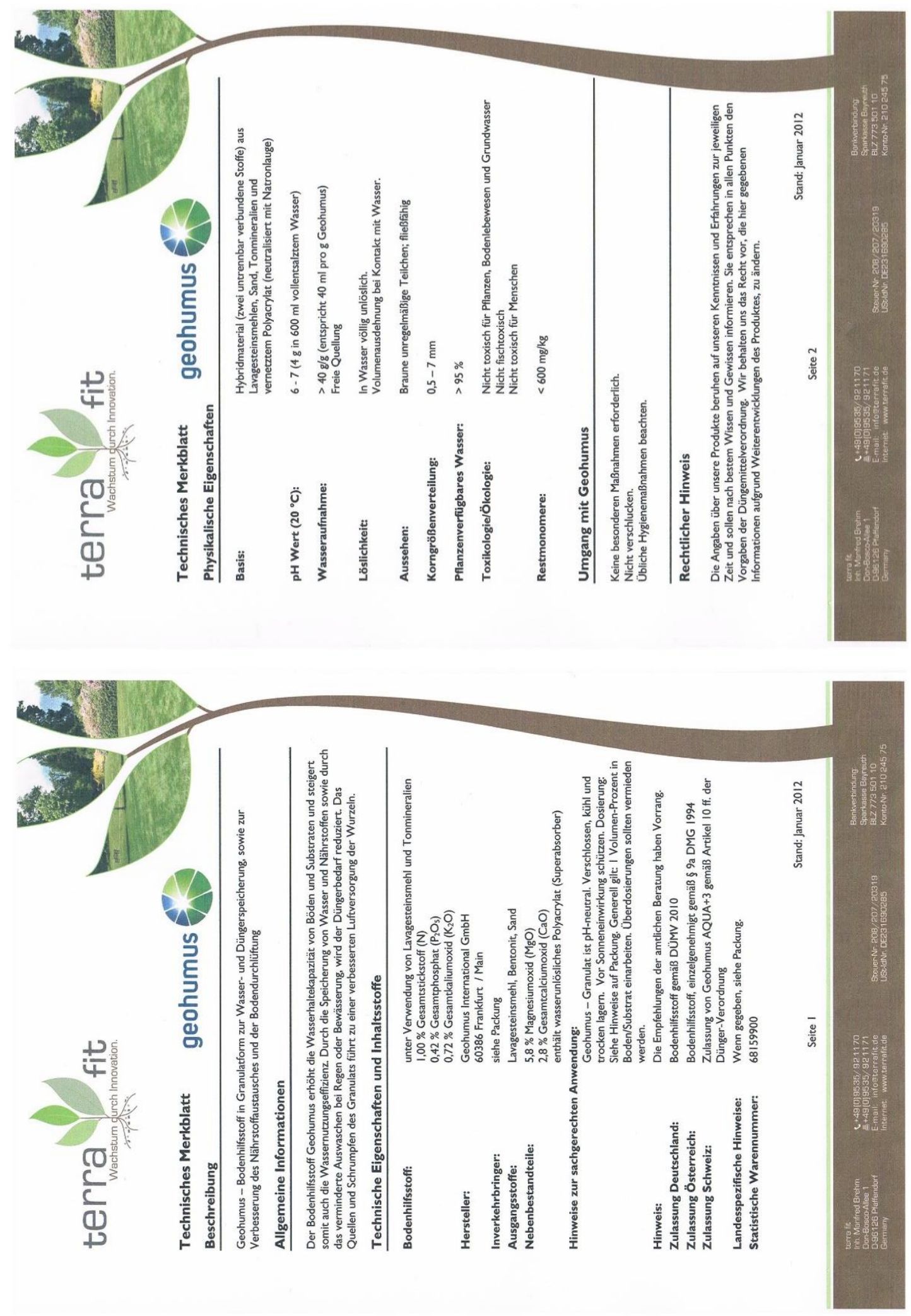
Abb. 50: Informationsblatt zum Mykorrhizapilz-Konzentrat.
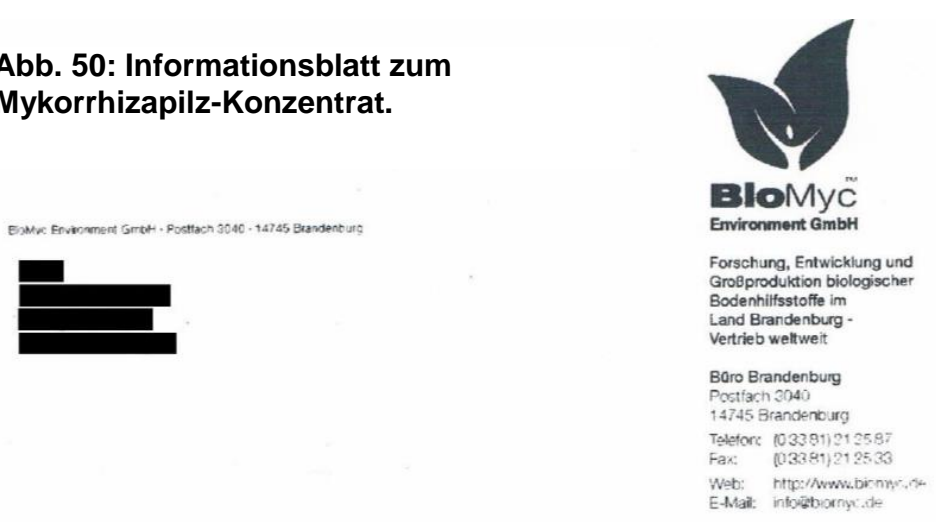

Brandenburg, 2015-05-13

Kunden-Nr.

Rechnungs-Nr.

Vielen Dank für Ihre Bestellung .

Wir berechnen Ihnen:

\begin{tabular}{|c|c|}
\hline Artikel: & Mykorrhizapilz-Konzentrat \\
\hline Bezeichnung: & BioMyc ${ }^{\mathrm{TM}}$ Vital \\
\hline Verpackungsinhalt: & 25 Liter zzügl 5 Liter Bonus \\
\hline Einzelpreis pro Liter: & $6,76 €$ incl. $7 \%$ MWST \\
\hline Bezeichnung: & BioMyc ${ }^{r M}$ Vital \\
\hline Einzelpreis pro Liter: & $4,98 \in$ incl. $7 \%$ MWST \\
\hline Verpackungsinhalt: & 50 Liter zzügl. 10 Liter Bonus \\
\hline Rechnungsendpreis: & $418,00 \in$ incl. $7 \%$ MWST $27,35 €$ \\
\hline Frühjahrsbonus: & 15 Liter \\
\hline
\end{tabular}

Nach Zahlungseingang wird die Ware sofort versendet.

Abb. 51: Karten zu den Versuchsflächen in Brandenburg, Föhr und Göttingen.

\begin{tabular}{|llll|}
\hline Legende: & \multicolumn{3}{l|}{} \\
Pe & Paulownia elongata & Pa & Pappelklon Max 4 \\
Pf & Paulownia fortunei & Ro & Robinia pseudoacacia \\
Pt & Paulownia tomentosa & We & Weidenklon Tordis \\
\cline { 4 - 5 } M & Mykorrhiza & weiß & Paulownia \\
G & Geohumus & blau & Pappel \\
N & Normal = Nullfläche & rot & Robinie \\
& & gelb & Weide \\
\hline & & Position der Wetterstation & \\
\hline
\end{tabular}

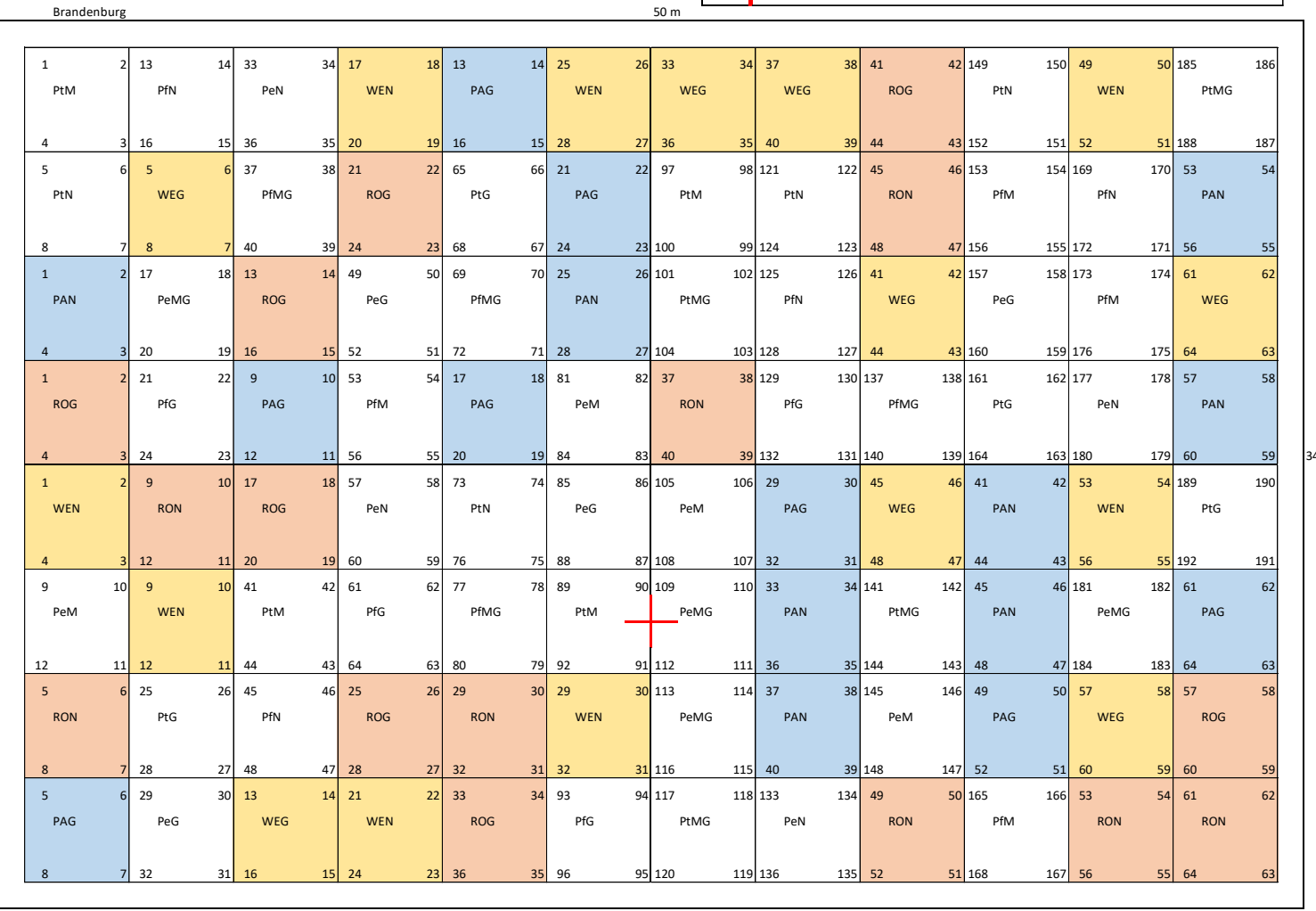



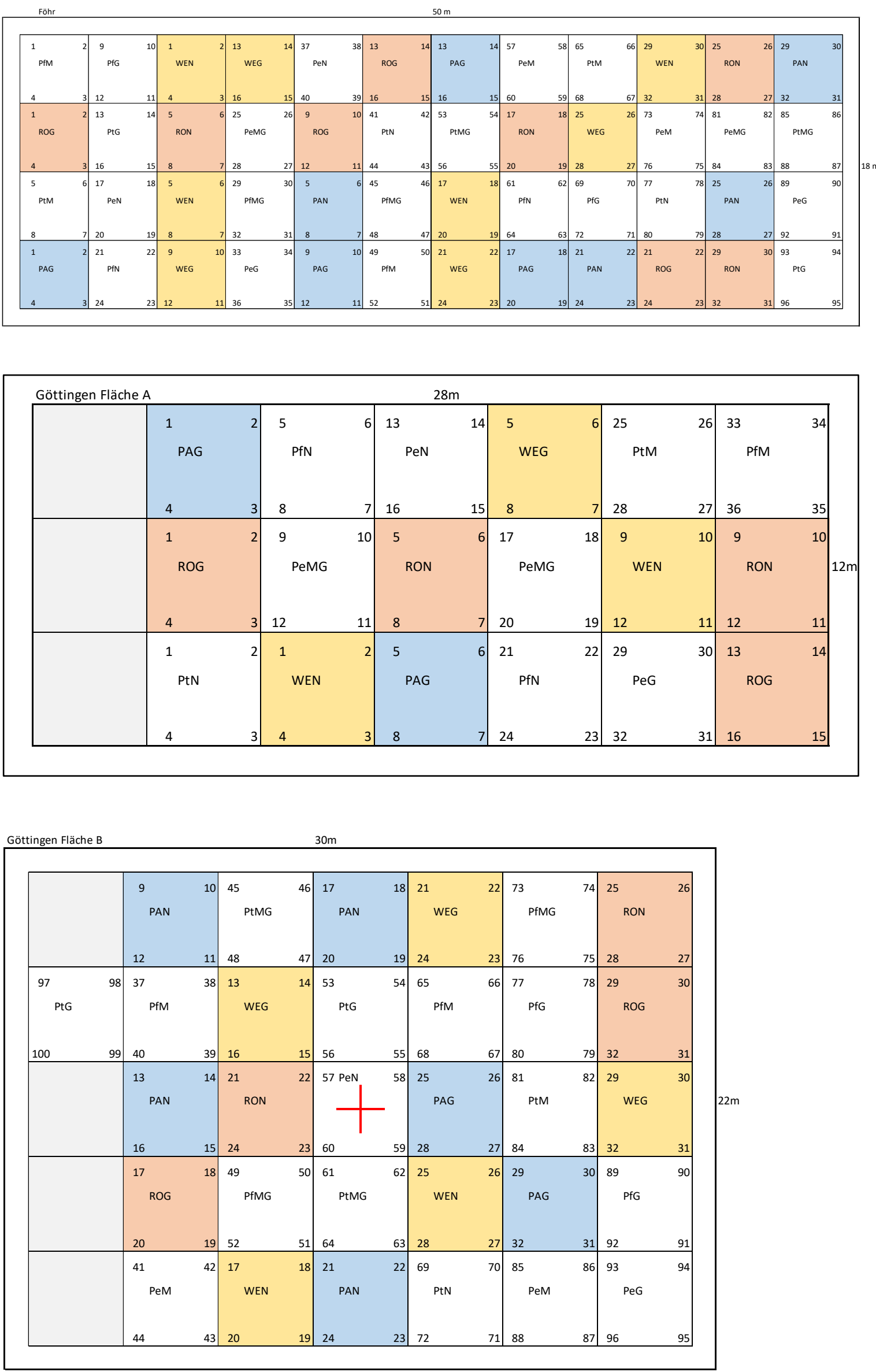
Tab. 13: Tabellarische Auflistung der Signifikanzen (Mittelwertvergleiche) zwischen den verschiedenen Baumarten und Behandlungsarten, unterteilt nach den Vegetationsperioden 2015, 2016 und 2017 und nach den Standorten Brandenburg (BB) und Göttingen (Gö). Leere Felder zeigen keine Signifikanzen. Graue Felder markieren fehlende Daten wegen der hohen Ausfallraten.

Voraussetzungen für den Post-hoc-Test: es handelt sich um ungleiche Stichprobenumfänge mit gleichen Varianzen (siehe Levene-Test zur Homogenität der Varianzen) Da der Stichprobenumfang eine große Differenz aufweist ( $\mathrm{N}$ von 3 bis 14 ) findet der Hochbergs GT2 - Test Anwendung.
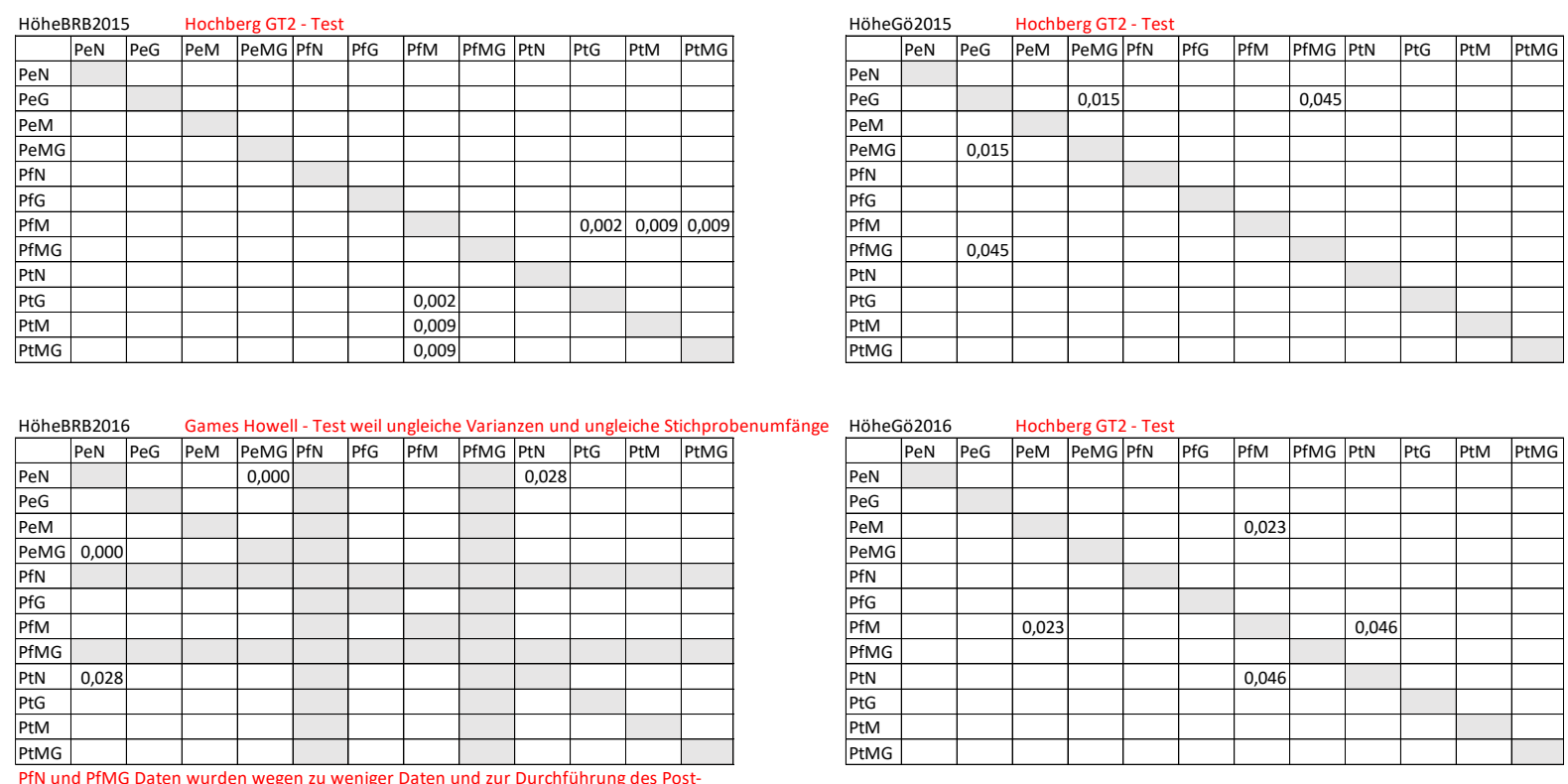

hoc-Test (mindestens 2 Werte nötig) aus der Berechnung entfernt
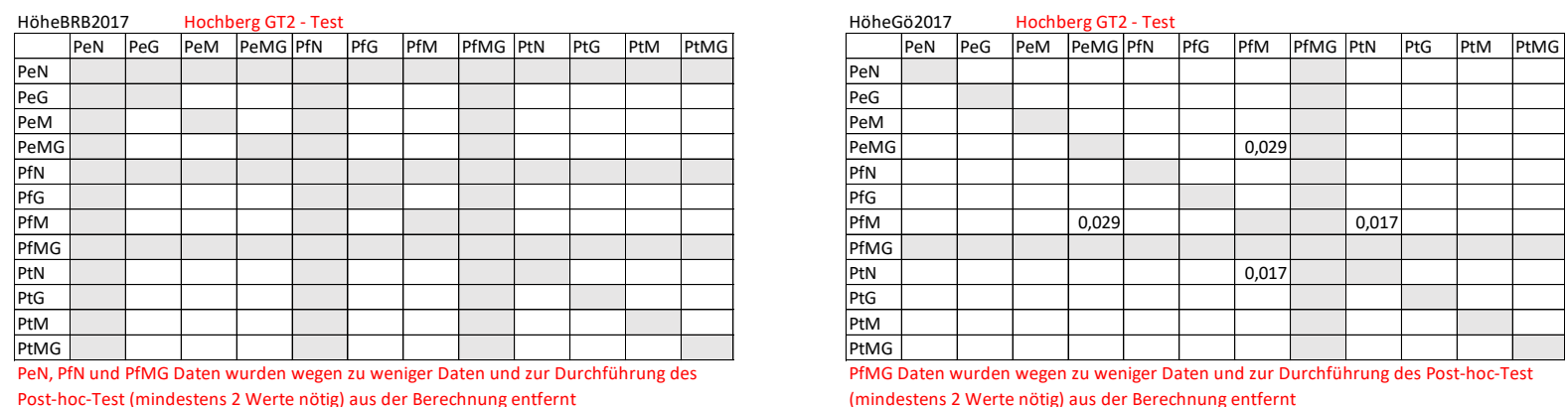


\section{Literaturverzeichnis}

Adolphi, K., 1997. Neophytische Kultur- und Anbaupflanzen als Kulturflüchtlinge des Rheinlandes, 1. Nachtrag. Osnabrücker Naturwissenschaftliche Mitteilungen, Band 23, S. 27-36 11.

Ammer, C., Arenhövel, W., Bauhus, J., Bolte, A., Degen, B., Dieter, M., Erhart, H.P., Erler, J., Hein, S., Kätzel, R., Konnert, M., Leder, B., Mosandl, R., Spellmann, H., Schölch, M., Schmidt, O., Schmidt, W., Schmitt, U., Spathelf, P., Teuffel, K. v, Vor, T., 2014.

Erhebliche Zweifel an der naturschutzfachlichen Invasivitätsbewertung einiger forstlich relevanter Baumarten - offener Brief. AFZ-Der Wald 69 (14), 12-14.

APA, 1991. American Paulownia Association [WWW Document]. URL https://paulowniatrees.org/ (accessed 3.5.20).

APG III, 2009. An update of the Angiosperm Phylogeny Group classification for the orders and families of flowering plants: APG III. Botanical Journal of the Linnean Society 161, 105121. https://doi.org/10.1111/j.1095-8339.2009.00996.x

Bartels, H., 1993. Gehölzkunde: Einführung in die Dendrologie, UTB. Ulmer, Stuttgart.

Barton, I.L.S., 2007. Paulownia, FRI bulletin 1992-2007 ; ZDB-ID: 1326063-7. New Zealand Forest Research Institute, Rotorua.

BArtSchV, 2020. Bundesartenschutzverordnung (BArtSchV - nichtamtliches Inhaltsverzeichnis) der Bundesrepublik Deutschland, Neufassung 2005 [WWW Document]. URL https://www.gesetze-im-internet.de/bartschv_2005/index.html (accessed 7.1.20).

Bean, W.J., 1973. Trees and shrubs hardy in the British Isles, Vol. II, D-M.

Beasley, R.R., Pijut, P.M., 2010. Invasive plant species in hardwood tree plantations. FNR-230-W West Lafayette, IN: Purdue University Extension Service, Hardwood Tree Improvement Center. 25 p. FNR-230W, 1-25.

Bergström, R., Guillet, C., 2002. Summer browsing by large herbivores in short-rotation willow plantations. Biomass and Bioenergy 23, 27-32. https://doi.org/10.1016/S09619534(02)00027-2

Berry, S., 2005. Romanov prophecy. Ballantine Books, New York, NY.

BfN, 2020a. Neobiota: Rechtliche Rahmenbedingungen, Bundesamt für Naturschutz [WWW Document]. URL https://neobiota.bfn.de/grundlagen/rechtlicher-rahmen.html (accessed 7.1.20).

BfN, 2020b. Berner Konvention: Erhaltung wild lebender Pflanzen und Tiere und ihrer Lebensräume [WWW Document]. URL https://www.bfn.de/themen/artenschutz/regelungen/berner-konvention.html (accessed 6.24.20).

BfN, 2012. Daten zur Natur 2012, Bundesamt für Naturschutz. Landwirtschaftsverlag, Münster: $446 \mathrm{~S}$.

BLfL, 2003. (Bayrische Landesanstalt für Landwirtschaft): Streuobst in der Kulturlandschaft. Fachtagung des Institutes für Agrarökologie, ökologischen Landbau und Bodenschutz zusammen mit der Bayerischen Landesanstalt für Weinbau und Gartenbau am 09./10.07.2003 in Kirchehrenbach, Landkreis Forchheim. Tagungsband, Schriftenreihe der LfL, $85 \mathrm{~S}$.

BMEL, 2017. BWaldG - Gesetz zur Erhaltung des Waldes und zur Förderung der Forstwirtschaft Bundesministerium für Ernährung und Landwirtschaft [WWW Document]. URL https://www.gesetze-im-internet.de/bwaldg/BJNR010370975.html (accessed 7.6.20).

BNatSchG, 2009. Gesetz über Naturschutz und Landschaftspflege (Bundesnaturschutzgesetz). Verkündet 29.07.2009, BGBI. I S. 2542, das zuletzt durch Artikel 1 des Gesetzes vom 4.März 2020 (BGBI. I S. 440) geändert worden ist; Inkrafttreten am 01.03.2010.

Boelcke, B., 2007. Grundsätze des Anbaus schnellwachsender Baumarten und Ertragspotenziale in Mecklenburg-Vorpommern. Vortrag zur Fachtagung „Energieholzproduktion auf 
landwirtschaftlichen Flächen“, 9. Mai 2007 in Güstrow. www.agrarnetmv.de/index.php?/content/view/ full/3363, Stand 05.08.08.

Böhm, C., Tsonkova, P., Zehlius-Eckert, W., 2017. Wie können Agroforstsysteme praktikabel in das deutsche Agrarförderrecht eingebunden werden? In: Tagungsband. Mit Beiträgen des 5. Forums Agroforstsysteme 30.11. bis 01.12.2016 in Senftenberg (...). Bäume in der Land(wirt)schaft - von der Theorie in die Praxis. Herausgeber: Dr. Christian Böhm Brandenburgische Technische Universität Cottbus-Senftenberg Fachgebiet für Bodenschutz und Rekultivierung. http://agroforst-info.de/wpcontent/uploads/2017/03/Tagungsband_5_Forum_Agroforstsysteme_.pdf.

Bonner, F.T., 1990. Paulownia tomentosa (Thunb.) Sieb. \& Zucc. ex Steud. Royal Paulownia 2.

Boote, D.N., Beile, P., 2005. Scholars Before Researchers: On the Centrality of the Dissertation Literature Review in Research Preparation. Educational Researcher 34, 3-15. https://doi.org/10.3102/0013189X034006003

Bork, J., Walter, E., Mosandl, R., Stimm, B., 2015. Paulownia - vorläufige Ergebnisse zu Möglichkeiten und Grenzen des Anbaus im Wald. Forstarchiv 102-106. https://doi.org/10.4432/0300-4112-86-102

Bourier, G., 2013. Beschreibende Statistik: Praxisorientierte Einführung. Springer-Verlag.

Brandle, J.R., Hodges, L., Zhou, X.H., 2004. Windbreaks in North American agricultural systems, in: Nair, P.K.R., Rao, M.R., Buck, L.E. (Eds.), New Vistas in Agroforestry: A Compendium for 1st World Congress of Agroforestry, 2004, Advances in Agroforestry. Springer Netherlands, Dordrecht, pp. 65-78. https://doi.org/10.1007/978-94-017-2424-1_5

Brix, M., Bender, B., Spiecker, H., 2009. Wertholzproduktion in Agroforstsystemen, in: Reeg, T., A. Bemmann, W. Konold, D. Murach und H. Spiecker (Hrsg.), Anbau und Nutzung von Bäumen auf landwirtschaftlichen Flächen, John Wiley \& Sons, Weinheim. p. 1.

Brodtbeck, T., Zemp, M., Frei, M., Kienzle, U., Knecht, D., 1999. Flora von Basel und Umgebung 1980-96. Sonderdruck der Mitteilungen der Naturforschenden Gesellschaften bei der Basel 2: 543-1003.

Browne, D.J., 1851. The Trees of America: Native and Foreign, Pictorially and Botanically Delineated, and Scientifically and Popularly Described ... Illustrated by Numerous Engravings. Harper \& brothers.

Büker, F., 2015. Jugendwachstum und Trockenresistenz von verschiedenen Paulownia-Arten, Bachelorarbeit an der Fakultät für Forstwissenschaften und Waldökologie der GeorgAugust-Universität Göttingen, unveröffentlicht.

Butin, H., 2011. Krankheiten der Wald- und Parkbäume. Leitfaden zum Bestimmen von Baumkrankheiten. Georg Thieme Verlag Stuttgart. New York.

Buttler, K.P., Thieme, M., 2013. Florenliste von Deutschland - Gefäßpflanzen [WWW Document]. URL https://www.kp-buttler.de/florenliste/index.htm (accessed 5.13.20).

Cabi, 2019. Invasive Species Compendium: Paulownia tomentosa [WWW Document]. URL https://www.cabi.org/isc/datasheet/39100 (accessed 5.12.20).

Cathaia, 2020. Paulownia Baumschule Schröder, eine Marke der Cathaia International GmbH\&Co.KG, Orleansstr. 2, 81669 München. [WWW Document]. URL http://www.paulownia-baumschule.de/ (accessed 7.21.20).

CBD Secretariat (UN), 2020. The Convention on Biological Diversity [WWW Document]. URL https://www.cbd.int/convention/ (accessed 6.24.20).

CBD Secretariat (UN), 2002. Alien species: guiding principles for the prevention, introduction and mitigation of impacts - United Nations Environment Probramme [WWW Document]. URL https://www.cbd.int/kb/record/recommendation/7021?Event=SBSTTA-05 (accessed 6.24.20).

Chalmin, A., 2009. Produktionsaspekte in Agroforstsystemen mit Werthölzern landwirtschalftliche Produktion, in: Reeg, T., A. Bemmann, W. Konold, D. Murach und H. Spiecker (Hrsg.), Anbau und Nutzung von Bäumen auf landwirtschaftlichen Flächen, John Wiley \& Sons, Weinheim. p. 1. 
Chalmin, A., Möndel, A., 2009. Rechtliche Rahmenbedingungen für Agroforstsysteme, in: Reeg, T., A. Bemmann, W. Konold, D. Murach und H. Spiecker (Hrsg.), Anbau und Nutzung von Bäumen auf landwirtschaftlichen Flächen, John Wiley \& Sons, Weinheim. p. 1.

Chao, Y., Chen, Y., 1980. Economic insect fauna of China. Fasc. 20 Coleoptera. Curculionidae (I). Science Press. Beijing, China, p. 184.

Chen, S., Xie, Y., Deng, G., 1959. Economic insect fauna of China. Fasc. 1, in: Coleoptera. Cerambycidae. Science Press. Beijing, China, p. 120.

CITES.org, 2020. Washingtoner Artenschutzübereinkommen (CITES = Convention on International Trade in Endangered Species of Wild Fauna and Flora, von 1973) [WWW Document]. URL https://www.cites.org/ (accessed 7.1.20).

Clay, D.V., Dixon, F.L., 1997. Effect of ground-cover vegetation on the growth of poplar and willow short-rotation coppice. Aspects of Applied Biology 53-60.

Consilio Floraum, 1987. Consilio Floraum Cryptogamarum Sinicarum Academiae Sinicae. Erysiphales, Science Press, Beijing, China.

Cook, D.J., Mulrow, C.D., Haynes, R.B., 1997. Systematic Reviews: Synthesis of Best Evidence for Clinical Decisions. Annals of Internal Medicine 126, 376-380. https://doi.org/10.7326/0003-4819-126-5-199703010-00006

Cotta, H. von, 1819. Die Verbindung des Feldbaues mit dem Waldbau oder die Baumfeldwirthschaft. Arnold'sche Buchhandlung, Dresden, $56 \mathrm{~S}$.

Dai, F., 1979. Sylloge Fungorum Sinicorum. Science Press. Beijing, China, p. 1527.

Dannenberg, F.S.M., 2016. Jugendwachstum von Paulownia elongata, fortunei und tomentosa bei unterschiedlicher Lichtintensität, Masterarbeit an der Fakultät für Forstwissenschaften und Waldökologie der Georg-August-Universität Göttingen, unveröffentlicht.

Datenblatt geohumus.pdf [WWW Document], n.d. URL http://www.terrafit.de/media/products/Datenblatt\%20geohumus.pdf (accessed 9.21.15).

David, H., 2012. Paulownia - empress tree [WWW Document]. Growing History. URL https://growinghistory.wordpress.com/category/paulownia-empress-tree/ (accessed 3.2.20).

DB, 2017. Regelungen zu Kurzumtriebsplantagen, Aktenzeichen WD 5 - 3000 - 087 / 17, Fachbereich: WD5: Wirtschaft und Verkehr, Ernährung, Landwirtschaft und Verbraucherschutz. Wissenschaftlicher Dienst des Deutschen Bundestages.

Dening, K., 1937. Zur Kenntnis von Paulownia tomentosa (Thunb.) Steud. Mitt. Dtsch. Dendrol. Ges. 49, 127-129.

Dickmann, D.I., 2006. Silviculture and biology of short-rotation woody crops in temperate regions: Then and now. Biomass and Bioenergy, Proceedings of the 5th Biennial meeting of the Short Rotation Woody Crops Operations Working Group in conjunction with International Energy Agency Bioenergy, Task 30, Short Rotation Crops for Bioenergy Systems and International Union of Forest Research Organization, Working Unit 1.09.01, Integrated Research in Temperate Short-Rotation Energy Plantations 30, 696-705. https://doi.org/10.1016/j.biombioe.2005.02.008

DirektZahIDurchfV, 2020. Verordnung zur Durchführung der Direktzahlungen an Inhaber landwirtschaftlicher Betriebe im Rahmen von Stützungsregelungen der Gemeinsamen Agrarpolitik (Direktzahlungen-Durchführungsverordnung - DirektZahlDurchfV) § 30 Flächen mit Niederwald mit Kurzumtrieb (Artikel 46 Absatz 2 Buchstabe g der Verordnung (EU) Nr. 1307/2013): [WWW Document]. URL https://www.gesetze-iminternet.de/direktzahldurchfv/_30.html (accessed 7.8.20).

Dirlmeier, U., 1982. Review of Deutsche Agrargeschichte. Vom Neolithikum bis zur Schwelle des Industriezeitalters (Wissenschaftliche Paperbacks 12, Sozial- und Wirtschaftsgeschichte). Zeitschrift für Historische Forschung 9, 476-478.

DMN, 1975. This tree a shade versatile, Dallas Morning News. Sect. C:9. 
Döpke, K., Moschner, C.R., Hartung, E., 2013. Die ökologischen Aspekte von Kurzumtriebsplantagen: eine Literaturstudie. Landtechnik, 33-37.

Drew, A.P., Zsuffa, L., Mitchell, C.P., 1987. Terminology relating to woody plant biomass and its production. Letter to the Editor. Biomass 12: 79 - 82.

DVFFA, 2020. Deutscher Verband Forstlicher Forschungsanstalten [WWW Document]. URL http://www.dvffa.de/ (accessed 3.12.21).

DWD, 2020. (DWD) Niederschlag: langjährige Mittelwerte 1981 - 2010. von: http://www.dwd.de/DE/leistungen/klimadatendeutschland/mittelwerte/nieder_8110_ akt_html.html?view=nasPublication\&nn=16102; abgerufen Januar 2016.

Eckardt, D., 2018. Reaktionen von zweijährigen Stockausschlägen der Arten Paulownia elongata, fortunei und tomentosa auf unterschiedliche Beschattung, Bachelorarbeit an der Fakultät für Forstwissenschaften und Waldökologie der Georg-August-Universität Göttingen, unveröffentlicht.

eFloras, 2014. Flora of China. Missouri Botanical Garden, St. Louis, MO \& Harvard University Herbaria, Cambridge, MA. [WWW Document]. URL http://www.efloras.org/florataxon.aspx?flora_id=2\&taxon_id=124177 (accessed 5.12.20).

Eichhorn, M.P., Paris, P., Herzog, F., Incoll, L.D., Liagre, F., Mantzanas, K., Mayus, M., Moreno, G., Papanastasis, V.P., Pilbeam, D.J., Pisanelli, A., Dupraz, C., 2006. Silvoarable Systems in Europe - Past, Present and Future Prospects. Agroforest Syst 67, 29-50. https://doi.org/10.1007/s10457-005-1111-7

Einheitserde, 2020. Einheitserde Werkverband e.V. URL https://www.einheitserde.de/produkte/spezial-substrate/ (accessed 3.16.21).

Elton, C.S., Pyšek, P., Richardson, D.M., 1958. The ecology of invasions by animals and plants. London: Methuen. Progress in Physical Geography: Earth and Environment 31, 659666. https://doi.org/10.1177/0309133307087089

EnPf, 2021. EnergiePflanzen.com: Paulownia-online-kaufen, Paulownia, Kiribaum, Blauglockenbaum oder Kaiserbaum. Energiepflanzen. URL https://www.energiepflanzen.com/paulownia/ (accessed 2.27.21).

Essel, F., 2007. From ornamental to detrimental? The incipient invasion of Central Europe by Paulownia tomentosa. Preslia 79, 377-389.

EU-Biodiversitätsstrategie, 2011. Biologische Vielfalt in Europa, Einzelziele der EUBiodiversitätsstrategie für 2020, Europäische Kommission [WWW Document]. Bundesministerium für Umwelt, Naturschutz und nukleare Sicherheit. URL https://www.bmu.de/themen/natur-biologische-vielfalt-arten/naturschutzbiologische-vielfalt/biologische-vielfalt-international/biologische-vielfalt-in-europa/ (accessed 7.1.20).

Executive Order 13112, 1999. Executive Orders for Invasive Species | National Invasive Species Information Center | USDA [WWW Document]. URL https://www.invasivespeciesinfo.gov/executive-orders-invasive-species (accessed 3.6.20).

Fang, C., 2000. Fauna Sinica, Insects. Vol. 19. Lepidoptera, Arctiidae. Science Press. Beijing, China, p. 589.

Felbermeier, B., Höllerl, S., Stimm, B., Abt, A., Binder, F., Kateb, H.E., Mosandl, R., 2015. Waldbauliche Versuche in Bayern; LWF Wissen, 76. 18.

FFH-Richtlinie, 1992. Richtlinie 92/43/EWG des Rates vom 21. Mai 1992 zur Erhaltung der natürlichen Lebensräume sowie der wildlebenden Tiere und Pflanzen, 206.

Flora Japonica, 1835. Flora Japonica, Sectio Prima (Textband I) (1835) [WWW Document]. URL http://www.biolib.de/siebold/flora1/high/CRW_6270_RT8.html (accessed 3.8.20).

Foster, S., Duke, J.A., 2000. A Field Guide to Medicinal Plants and Herbs of Eastern and Central North America. Houghton Mifflin Harcourt.

Friedrich, E., 1999. Anbautechnische Untersuchungen in forstlichen Schnellwuchsplantagen und Demonstration des Leistungsvermögens schnellwachsender Baumarten. In: Hofmann, 
M. (Hrsg.): Modellvorhaben „Schnellwachsende Baumarten“ - Zusammenfassender Abschlussbericht. Schriftenreihe Nachwachsende Rohstoffe 13: 19-150.

Geohumus, 2020. Home [WWW Document]. Geohumus GmbH. URL https://www.geohumus.com/ (accessed 8.25.20).

Gerhardt, P., 2021. Baumfeldwirtschaft.de: Agroforst, Keyline Design, ReLaWi. URL http://baumfeldwirtschaft.de/ (accessed 2.28.21).

Gerold, D., Landgraf, D., Wolf, H., Schildbach, M., 2009. Bewirtschaftungsstrategien von Kurzumtriebsplantagen, in: Reeg, T., A. Bemmann, W. Konold, D. Murach und H. Spiecker (Hrsg.), Anbau und Nutzung von Bäumen auf landwirtschaftlichen Flächen, John Wiley \& Sons, Weinheim. p. 1.

Gordon, A.M., Newman, S.M., Coleman, B., 2018. Temperate Agroforestry Systems. CABI. Gradmann, R., 1950. Das Pflanzenleben der Schwäbischen Alb. 4. Auflage. Kohlhammer, Stuttgart, $449 \mathrm{~S}$.

Grünewald, H., Reeg, T., 2009. Überblick über den Stand der Forschung zu Agroforstsystemen in Deutschland, in: Reeg, T., A. Bemmann, W. Konold, D. Murach und H. Spiecker (Hrsg.), Anbau und Nutzung von Bäumen auf landwirtschaftlichen Flächen, John Wiley \& Sons, Weinheim. p. 1.

Hall, T., 2008. Trees for Life Journal - Paulownia: An Agroforestry Gem [WWW Document]. URL https://www.tfljournal.org/article.php/20080418100402327 (accessed 3.5.20).

Hao, Z., Yun, W., Jianqing, D., Denise, D., Weidong, F., Reardon, R., 2004. Invasive Plants of Asian Origin Established in the United States and Their Natural Enemies. U.S. Department of Agriculture, Forest Service, Forest Health Technology Enterprise Team.

Härtel, C., 2007. Agroforstsysteme. Fast nebenbei: Wertholz vom Acker. Schweizer Holz-Börse 32: 36-38 [WWW Document]. URL https://scholar.google.de/scholar?hl=de\&as_sdt=0\%2C5\&q=Agroforstsysteme. + Fast $+n$ ebenbei\%3A+Wertholz+vom+Acker\&btnG= (accessed 7.29.20).

Hecker, U., Weisgerber, H., 2014. Paulownia tomentosa, in: Enzyklopädie der Holzgewächse: Handbuch und Atlas der Dendrologie. American Cancer Society, pp. 1-10. https://doi.org/10.1002/9783527678518.ehg2003018

Helbig, C., Müller, M., 2009. Abiotische und biotische Schadfaktoren in Kurzumtriebsplantagen, in: Reeg, T., A. Bemmann, W. Konold, D. Murach und H. Spiecker (Hrsg.), Anbau und Nutzung von Bäumen auf landwirtschaftlichen Flächen, John Wiley \& Sons, Weinheim. p. 1.

Helbig, C., Müller, M., 2008. Potenzielle biotische Schadfaktoren in Kurzumtriebsplantagen. Cottbuser Schriften zur Ökosystemgenese und Landschaftsentwicklung 6: 101-116.

Herzog, F., 1998. Streuobst: a traditional agroforestry system as a model for agroforestry development in temperate Europe. Agroforestry Systems 42, 61-80. https://doi.org/10.1023/A:1006152127824

Herzog, F., 1997. Konzeptionelle Überlegungen zu Agroforstwirtschaft als Landnutzungsalternative in Europa. Zeitschrift für Kulturtechnik und Landentwicklung 38 (1), 32-35 [WWW Document]. URL https://scholar.google.de/scholar?hl=de\&as_sdt=0\%2C5\&q=Konzeptionelle+\%04berleg ungen+zu+Agroforstwirtschaft+als+Landnutzungsalternative+in+Europa\&btnG= (accessed 7.21.20).

Hoffmann, C.I., 2019. Biomassenzuwachs von Jungpflanzen der Arten Paulownia elongata, Paulownia fortunei und Paulownia tomentosa unter modifizierten Standortbedingungen, Masterarbeit an der Fakultät für Forstwissenschaften und Waldökologie der Georg-August-Universität Göttingen, unveröffentlicht.

Hoffmann, C.I., 2017. Reaktionen von zweijährigen Paulownia-Stockausschlägen auf unterschiedliche Wasserversorgung, Bachelorarbeit an der Fakultät für Forstwissenschaften und Waldökologie der Georg-August-Universität Göttingen, unveröffentlicht. 
Hofheinz, F., 2016. Umtriebszeiten von Kurzumtriebsplantagen, Bachelorarbeit an der Fakultät für Forstwissenschaften und Waldökologie der Georg-August-Universität Göttingen, unveröffentlicht.

Hofmann, M., 2007. Energieholzproduktion in der Landwirtschaft. Fachagentur Nachwachsende Rohstoffe e. V., $42 \mathrm{~S}$.

Hoppe, J.R., 2020. Allgemeine Botanik, Vorlesung Bio.0001.003, 5. Anatomie des Blattes - Ulm University [WWW Document]. URL https://www.uniulm.de/en/nawi/sysbot/lehre/grundlagen/allgemeinebotanik/blattanatomie/ (accessed 3.13.20).

Hu, S.-Y., 1961. The economic botany of the paulownias. Econ Bot 15, 11-27. https://doi.org/10.1007/BF02906759

Hu, S.Y., 1959. A monograph of the genus Paulownia. Quarterly Journal of the Taiwan Museum $12,1-54$.

Huang, F., 1993. Insects of Wuling Mountains Area, Southwestern China. Science Press. Beijing, China, p. 777.

Hubo, C., Jumpertz, E., Krott, M., Nockemann, L., Steinmann, A., Bräuer, I., 2007. Grundlagen für die Entwicklung einer nationalen Strategie gegen invasive gebietsfremde Arten. Bundesamt für Naturschutz, BfN-Skripten 213, p. 387.

Innes, R.J., 2009. Paulownia tomentosa. In: Fire Effects Information System. U.S. Department of Agriculture, Forest Service, Rocky Mountain Research Station, Fire Sciences Laboratory [WWW Document]. URL https://www.fs.fed.us/database/feis/plants/tree/pautom/all.html (accessed 3.5.20).

International Plant Names Index [WWW Document], 2020. URL https://www.ipni.org/?f=f_specific\%2Cf_infrageneric\%2Cf_infrafamilial\%2Cf_familial\% 2Cf_infraspecific\%2Cf_generic\&q=paulownia (accessed 3.26.20).

Johnson, J.E., Mitchem, D.O., Kreh, R.E., 2003. Establishing royal paulownia on the Virginia Piedmont. New Forests 25, 11-23. https://doi.org/10.1023/A:1022349231557

Johnson, K., 2005. Fact Sheet: Princess Tree (SUGGESTED ALTERNATIVE PLANTS), Plant Conservation Alliance ${ }^{\circledR}$ S Alien Plant Working Group http://www.nps.gov/plants/alien/.

Kays, J., 1996. How to Produce \& Market Paulownia [WWW Document]. URL https://www.docdeveloppement-durable.org/file/Arbres-Bois-de-RapportReforestation/FICHES_ARBRES/Paulownia/How\%20to\%20Produce\%20\&\%20Market\%2 OPaulownia.pdf (accessed 3.5.20).

Keil, P., Loos, G.H., 2004. Ergasiophygophyten auf Industriebrachen des Ruhrgebietes. Floristische Rundbriefe, 38, Bochum.

Keller, S., Häni, F., 2000. Ansprüche von Nützlingen und Schädlingen an den Lebensraum. In: W. Nentwig (Hrsg.): Streifenförmige ökologische Ausgleichsflächen in der Kulturlandschaft: Ackerkrautstreifen, Buntbrache, Feldränder. Bern Hannover, vaö Verlag Agrarökologie: 199-217.

Kiermeier, P., 1977. Erfahrungen mit Paulownia tomentosa (Thunb. Steud. im Rheingau. Mitt. Dtsch. Dendrol. Ges. 69, 11-22.

Knust, C., 2009. Kurzumtriebsplantagen - Stand des Wissens, in: Reeg, T., A. Bemmann, W. Konold, D. Murach und H. Spiecker (Hrsg.), Anbau und Nutzung von Bäumen auf landwirtschaftlichen Flächen, John Wiley \& Sons, Weinheim. p. 1.

Kobayashi, S., Asai, T., Fujimoto, Y., Kohshima, S., 2008. Anti-herbivore Structures of Paulownia tomentosa: Morphology, Distribution, Chemical Constituents and Changes During Shoot and Leaf Development. Ann Bot 101, 1035-1047. https://doi.org/10.1093/aob/mcn033

König, W., Radkau, J., 2013. Technik in Deutschland. Vom 18. Jahrhundert bis zur Gegenwart, 1989, in: Nachdenken Über Technik. Nomos Verlagsgesellschaft mbH \& Co. KG, pp. 309-312. https://doi.org/10.5771/9783845269238-309 
Konold, W., Reeg, T., 2009. Historische Agroforstsysteme in Deutschland, in: Reeg, T., A. Bemmann, W. Konold, D. Murach und H. Spiecker (Hrsg.), Anbau und Nutzung von Bäumen auf landwirtschaftlichen Flächen, John Wiley \& Sons, Weinheim. p. 1.

Kowarik, I., 2010. Biologische Invasionen. Neophyten und Neozoen in Mitteleuropa., 2 erweiterte Auflage. ed. Ulmer Verlag.

Kowarik, I., 1995. Time lags in biological invasions with regard to the success and failure of alien species. Pyšek, P., Prach, K., Rejmánek, M. \& Wade, M. (Eds.), Plant invasions. General aspects and special problems. SPB Academic Publ., Amsterdam 15-38.

Lacy, A., 2000. In a green schade: writings from homeground. New York, NY: Houghton Mifflin Company.

Landgraf, D., Ertle, C., Böcker, L., 2005. Wuchspotenzial von Stockausschlägen der Robinie auf Bergbaufolgeflächen. AFZ - Der Wald 14: 748 - 749.

Landolt, E., 1993. Über Pflanzenarten, die sich in den letzten 150 Jahren in der Stadt Zürich stark ausgebreitet haben. Phytocoenologia, Band 23, Heft 1-4 651-663. https://doi.org/10.1127/phyto/23/1993/651

Lee, T.B., 1983. Endemic plants and their distribution in Korea.

Lei, C., Zhou, Z., 1998. Insect Records of Hubei, China. Hubei Science and Technology Publishing House. Wuhan, China, p. 650.

Lenssen, N.J.L., Schmidt, G.A., Hansen, J.E., Menne, M.J., Persin, A., Ruedy, R., Zyss, D., 2019. Improvements in the GISTEMP Uncertainty Model. J. Geophys. Res. Atmos. 124, 63076326. https://doi.org/10.1029/2018JD029522

LexBio, 2020. Lexikon der Biologie; Spektrum Akademischer Verlag, Heidelberg [WWW Document]. URL https://www.spektrum.de/lexikon/biologie/ (accessed 3.11.21).

Liu, X., Guo, Y., 1998. Flora Fungorum Sinicorum, in: Pseudocercospora. Science Press. Beijing, China, p. 473.

Longbrake, A.C.W., 2001. Ecology and Invasive potential of Paulownia Tomentosa (Scrulariaceae) in a Hardwood Forest Landscape. Ohio University.

Machatschek, M., 2002. Laubgeschichten: Gebrauchswissen einer alten Baumwirtschaft, Speise- und Futterlaubkultur, Laubgeschichten. Böhlau Verlag, Wien, 544 S.

Mayr, H., 1906. Fremdländische Wald- und Parkbäume für Europa. Berlin.

McCracken, A.R., Dawson, W.M., 1997. Growing clonal mixtures of willow to reduce effect of Melampsora epitea var. epitea. European Journal of Forest Pathology 27, 319-329. https://doi.org/10.1111/j.1439-0329.1997.tb01086.x

Mehrotra, M.D., 1997a. Fantastic growth response of Paulownia to mycorrhization and fertilization, The Indian forester - a monthly journal of forestry and forest research, 123 873-875.

Mehrotra, M.D., 1997b. Diseases of Paulownia and their management, The Indian forester - a monthly journal of forestry and forest research, 123 67-72.

Mehrotra, M. D., 1996. Multiplication of VAMF on Paulownia - a veritable possibility, Indian Journal of Forestry 1996, 122, 9, pp 858-860.

Mehrotra, M.D., 1996. Multiplication of VAMF on Paulownia - a veritable possibility. The Indian Forester 858-860.

Mehrotra, M.D., Sachan, S.N., Mehotra, A., 1998. Effect of VAM, fertilizer and deoiled neem cake application on the growth of Paulownia tomentosa raised from root cuttings. Indian Journal of Forestry 111-114.

Mehrotra, M. D., Sachan, S.N., Mehrotra, A., 1998. Effect of VAM, fertilizer and deoiled neem cake application on the growth of Paulownia tomentosa raised from root cuttings, Indian Journal of Forestry Vol. 21, S. 111-14.

Meyer, M., 2012. The night parade of one hundred demons: a field guide to Japanese Yokai. unbekannt, unbekannt.

Meyerhof, J., 2014. Die Bedeutung der Niederwaldbewirtschaftung in ausgewählten Ländern Europas, Bachelorarbeit an der Fakultät für Forstwissenschaften und Waldökologie der Georg-August-Universität Göttingen, unveröffentlicht. 
Mezzalira, G., Colonna, M.B., 2002. Paulownia, a multifunctional wood variety. Informatore Agrario 58, 65-73.

Michaelis, F., 2013. Herstellung von Spanplatten auf Basis von Kiri-Holz = Production of particleboards based on kiri wood, 80 S., Masterarbeit an der Fakultät für Forstwissenschaften und Waldökologie der Georg-August-Universität Göttingen, unveröffentlicht.

Möndel, A., Brix, M., Chalmin, A., 2009. Ökonomische Bewertung von Agroforstsystemen, in: Reeg, T., A. Bemmann, W. Konold, D. Murach und H. Spiecker (Hrsg.), Anbau und Nutzung von Bäumen auf landwirtschaftlichen Flächen, John Wiley \& Sons, Weinheim. p. 1.

Moore, J.E., Lacey, E.P., 2009. A Comparison of Germination and Early Growth of Four Early Successional Tree Species of the Southeastern United States in Different Soil and Water Regimes. The American Midland Naturalist 162, 388-394. https://doi.org/10.1674/0003-0031-162.2.388

Mosandl, R., Stimm, B., 2015. Kurzportrait Blauglockenbaum (Paulownia tomentosa), http://www.waldwissen.net. 4.

Murach, D., Kindermann, C., Hirschl, B., Aretz, A., Schneider, B.U., Grünewald, H., Schultze, B., Quinkenstein, A., Bilke, G., Muchin, A., Eberts, J., Grundmann, P., Jochheim, H., Scherzer, J., Hagemann, H., 2007. Zukunftsrohstoff Dendromasse - Hintergrund und erste Ergebnisse des Verbundforschungsprojekts DENDROM. Forstarchiv 78: 88-94.

Nakai, T., 1949. Die Familie Paulowniaceae. Journal of Japanes Botany 24, S. 13.

Nehring, S., Essel, F., Klingstein, F., Nowack, C., Rabitsch, W., Stöhr, O., Wiesner, C., Wolter, C., 2010. Schwarze Liste invasiver Arten: Kriteriensystem und Schwarze Listen invasiver Fische für Deutschland und für Österreich. Bundesamt für Naturschutz, BfNSkripten 285.

Nehring, S., Essl, F., Rabitsch, W., 2015. Methodik der naturschutzfachlichen Invasivitätsbewertung für gebietsfremde Arten: Version 1.3 ; unter Verwendung von Ergebnissen aus den F+E-Vorhaben FKZ 80682 330, FKZ 3510860500 und FKZ 351186 0300 und FKZ 351486 0200, BfN-Skripten. Bundesamt für Naturschutz, BfN-Skripten 401, Bonn.

Nehring, S., Kowarik, I., Rabitsch, W., Essel, F. (Eds.), 2013. Naturschutzfachliche Invasivitätsbewertungen für in Deutschland wild lebende gebietsfremde Gefäßpflanzen: unter Verwendung von Ergebnissen aus den F+E-Vorhaben FKZ 80682 330, FKZ 3510860500 und FKZ 351186 0300, BfN-Skripten. Bundesamt für Naturschutz, BfN-Skripten 352, Bonn.

Neobiota, 2020. Neobiota: Was sind Neobiota? Was sind invasive Arten? [WWW Document]. URL https://neobiota.bfn.de/grundlagen/neobiota-und-invasive-arten.html (accessed 5.30.20).

Nitsch, H., Röder, N., Oppermann, R., Baum, S., Schramek, J., Gundlach, J., 2016. Naturschutzfachliche Ausgestaltung von Ökologischen Vorrangflächen. Merkblatt 6. Kurzumtriebsplantagen als Ökologische Vorrangflächen (ÖVF). Praxishandbuch. Gefördert durch das Bundesamt für Naturschutz mit Mitteln des Bundesministeriums für Umwelt, Naturschutz, Bau und Reaktorsicherheit. Institut für Ländliche Strukturforschung an der Goethe-Universität Frankfurt am Main (IfLS); http://p125587.mittwaldserver.info/fileadmin/user_upload/Abbildungen/Projekte/Han dbuch_OEVForsch/Handbuch_OEVForsch_mit_Merkblaettern.pdf.

Nobis, L., 2014. Anbaumöglichkeiten und Risiken von Paulownia spec., Bachelorarbeit an der Fakultät für Forstwissenschaften und Waldökologie der Georg-August-Universität Göttingen, unveröffentlicht.

o.V., 1992a. The comprehensive scientific expedition to the Qinghai-Xizang Plateau, Chinese Academy of Sciences, in: Insects of the Hengduan Mountains Region, Vol. 1. Science Press. Beijing, China, pp. 1-865. 
o.V., 1992b. The comprehensive scientific expedition to the Qinghai-Xizang Plateau, Chinese Academy of Sciences, in: Insects of the Hengduan Mountains Region, Vol. 2. Science Press. Beijing, China, pp. 867-1547.

Palma, J., Graves, A., Bregt, A., Bunce, R., Burgess, P., Garcia, M., Herzog, F., Mohren, G., Moreno, G., Reisner, Y., 2005. Integrating Soil Erosion and Profitability in the Assessment of Silvoarable Agroforestry at the Landscape Scale. Conference paper: International Farming Systems Association (IFSA) European Symposium on Farming and Rural Systems, Vila Real, 4.-7.4.2004: 817-827 12.

Petzold, R., Feger, K.-H., Schwärzel, K., 2009. Wasserhaushalt von Kurzumtriebsplantagen, in: Reeg, T., A. Bemmann, W. Konold, D. Murach und H. Spiecker (Hrsg.), Anbau und Nutzung von Bäumen auf landwirtschaftlichen Flächen, John Wiley \& Sons, Weinheim. p. 1.

Poetsch, J., 2001. Permakultur - Ideologie oder praktische Landwirtschaft? Seminararbeit zur Pflanzenproduktion, Fakultät für Agrarwissenschaften, Georg-August-Universität Göttingen, $26 \mathrm{~S}$.

Pyšek, P., Jarošík, V., 2005. Residence time determines the distribution of alien plants, in: Inderjit (Ed.), Invasive Plants: Ecological and Agricultural Aspects. Birkhäuser, Basel, pp. 77-96. https://doi.org/10.1007/3-7643-7380-6_5

Rabitsch, W., Gollasch, S., Isermann, M., Starfinger, U., Nehring, S., 2013. Erstellung einer Warnliste in Deutschland noch nicht vorkommender invasiver Tiere und Pflanzen. Bundesamt für Naturschutz, BfN-Skripten 331.

Radkau, J., 2007. Holz: Wie ein Naturstoff Geschichte schreibt (Stoffgeschichten). oekom verlag.

Rauer, G., Driesch, M. von den, Lobin, W., Ibisch, P.L., Barthlott, W., 2000. Beitrag der deutschen Botanischen Gärten zur Erhaltung der Biologischen Vielfalt und Genetischer Ressourcen - Bestandsaufnahme und Entwicklungskonzept. Landwirtschaftsverlag, Münster: $246 \mathrm{~S}$.

Reeg, T., Bemmann, A., Konold, W., Murach, D., Spiecker, H., 2009a. Anbau und Nutzung von Bäumen auf landwirtschaftlichen Flächen. Wiley-VCH, Weinheim.

Reeg, T., Hampel, J., Hohlfield, F., Mathiak, G., Rusdea, E., 2009b. Agroforstsysteme aus Sicht des Naturschutzes, in: Reeg, T., A. Bemmann, W. Konold, D. Murach und H. Spiecker (Hrsg.), Anbau und Nutzung von Bäumen auf landwirtschaftlichen Flächen, John Wiley \& Sons, Weinheim. p. 1.

Remaley, T., 2005. Non-native Plants - Rock Creek Park (U.S. National Park Service) [WWW Document]. URL https://www.nps.gov/rocr/learn/nature/non-native-plants.htm (accessed 3.6.20).

Richter, M., Böcker, R., 2001. Städtisches Vorkommen und Verbreitungstendenzen des Blauglockenbaumes (Paulownia tomentosa) in Südwestdeutschland. Mitteilungen der Deutschen Dendrologischen Gesellschaft 86, 125-132.

Rock, J., Lasch, P., Kollas, C., 2009. Auswirkungen von absehbarem Klimawandel auf Kurzumtriebsplantagen, in: Reeg, T., A. Bemmann, W. Konold, D. Murach und H. Spiecker (Hrsg.), Anbau und Nutzung von Bäumen auf landwirtschaftlichen Flächen, John Wiley \& Sons, Weinheim. Wiley-VCH, Weinheim, p. 1.

Röhle, H., 2013. Standortleistungsschätzung und Biomasseermittlung in Kurzumtriebsplantagen. Ein Beitrag zum Gedenkkolloquium anlässlich des 10. Todestages von Prof. Dr. Friedrich Franz am 27. Juli 2012. Aus dem Institut für Waldwachstum und Forstliche Informatik, TU Dresden. In: Allg. Forst- u. J-Ztg. 11/12. Online verfügbar unter http://waldwachstum.wzw.tum.de/fileadmin/publications/2013_Standortleistungsschaetzung_Roehle.pdf.

Rösler, S., 2003. Natur- und Sozialverträglichkeit des Integrierten Obstbaus. Arbeitsberichte des Fachbereichs Architektur, Stadtplanung, Landschaftsplanung 151, Universität Kassel, 166 S. PhD thesis. University of Kassel. 
Schildbach, M., Grünewald, H., Wolf, H., Schneider, B.-U., 2009. Begründung von Kurzumtriebsplantagen: Baumartenwahl und Anlageverfahren, in: Reeg, T., A. Bemmann, W. Konold, D. Murach und H. Spiecker (Hrsg.), Anbau und Nutzung von Bäumen auf landwirtschaftlichen Flächen, John Wiley \& Sons, Weinheim. Wiley-VCH, Weinheim.

Schmidt, P.A., Gerold, D., 2008. Kurzumtriebsplantagen - Ergänzung oder Widerspruch zur nachhaltigen Waldwirtschaft? | Short-term rotation plantations - supplement or in contradiction to sustainable forest management? Schweizerische Zeitschrift fur Forstwesen 159, 152-157. https://doi.org/10.3188/szf.2008.0152

Schmidt, P.A., Glaser, T., 2009. Kurzumtriebsplantagen aus Sicht des Naturschutzes, in: Reeg, T., A. Bemmann, W. Konold, D. Murach und H. Spiecker (Hrsg.), Anbau und Nutzung von Bäumen auf landwirtschaftlichen Flächen, John Wiley \& Sons, Weinheim. p. 1.

Schmitz, U., Ristow, M., May, R., Bleeker, W., 2008. Hybridisierung zwischen Neophyten und heimischen Pflanzenarten in Deutschland. Nat. Landsch. 83: 444 - 451.

Scholz, V., Hellebrand, H.J., Höhn, A., 2004. Energetische und ökologische Aspekte der Feldholzproduktion. Bornimer Agrartechnische Berichte 35, 15-32.

Scholz, V., Lorbacher, F.R., Spikermann, H., 2009. Technologien der Ernte und Rodung von Kurzumtriebsplantagen, in: Reeg, T., A. Bemmann, W. Konold, D. Murach und H. Spiecker (Hrsg.), Anbau und Nutzung von Bäumen auf landwirtschaftlichen Flächen, John Wiley \& Sons, Weinheim. p. 1.

Scholz, V., Lücke, W., 2007. Stand der Feldholz-Erntetechnik. Landtechnik 62 (4): 222-223.

Setzer, R.J., 2019. Niederwaldumwandlung / - überführung am Beispiel der SIEGERLÄNDER Hauberge, Bachelorarbeit an der Fakultät für Forstwissenschaften und Waldökologie der Georg-August-Universität Göttingen, unveröffentlicht.

Shen, X., Shi, Z., 1998. The Fauna and Taxonomy of Insects in Henan. Vol. 2., in: Insects of the Funiu Mountains Region (I). China Agricultural Scientech Press, Beijing, China, p. 368.

Smiley, C.J., 1961. A Record of Paulownia in the Tertiary of North America. American Journal of Botany 48, 175-179. https://doi.org/10.1002/j.1537-2197.1961.tb11622.x

Snow, W.A., 2015. Ornamental, crop, or invasive? The history of the Empress tree (Paulownia) in the USA. Forests, Trees and Livelihoods 24, 85-96.

https://doi.org/10.1080/14728028.2014.952353

SRU, 2007. Sachverständigenrat für Umweltfragen: Sondergutachten Klimaschutz durch Biomasse. Sondergutachten des Sachverständigenrats für Umweltfragen ist ein wissenschaftliches Beratungsgremium der Bundesregierung, www.umweltrat.de/ 02gutach/downlo02/sonderg/SG_Biomasse_2007_Hausdruck.pdf [WWW Document].

Stimm, B., Stiegler, J., Genser, C., Wittkopf, S., Mosandl, R., 2013. Paulownia - Hoffnungsträger aus Fernost? - Eine schnellwachsende Baumart aus China in Bayern auf dem Prüfstand. LWF aktuell (96), 18-21. 4.

Stuber, M., Bürgi, M., 2002. Agrarische Waldnutzungen in der Schweiz 1800-1950. Nadel- und Laubstreue | Agricultural use of forests in Switzerland 1800-1950. Needles and leaves for litter harvesting. Schweizerische Zeitschrift fur Forstwesen 153, 397-410. https://doi.org/10.3188/szf.2002.0397

Tang, J., Yu, P., Li, H., Wang, S., 1980. Economic insect fauna of China. Fasc. 18 Coleoptera. Chrysomeloidea (I). Science Press. Beijing, China, p. 213.

Thielen, K., 2019. "Paulownia tomentosa - der Blauglockenbaum in der landwirtschaftlichen Nebennutzung auf Dauergrünland - Eine Beispielrechnung", Masterarbeit an der Fakultät für Forstwissenschaften und Waldökologie der Georg-August-Universität Göttingen, unveröffentlicht.

Thomasius, H., 1991. Anlage und Bewirtschaftung von Fichten-Holzproduktionsplantagen. In: Die Fichte, Bd. 2 Teil 3. Verlag Paul Parey, Hamburg, Berlin.

Unseld, R., 2009. Kombinierter Anbau von Wertholz- und Kurzumtriebsbäumen, in: Reeg, T., A. Bemmann, W. Konold, D. Murach und H. Spiecker (Hrsg.), Anbau und Nutzung von Bäumen auf landwirtschaftlichen Flächen, John Wiley \& Sons, Weinheim. p. 1. 
Unseld, R., Seidl, F., Nahm, M., Karopka, M., Zürcher, A., Möndel, A., 2014. Anlage und Bewirtschaftung von Kurzumtriebsflächen in Baden-Württemberg, 4. vollständig aktualisierte Auflage.

Visnjic, C., 2006. Aufforstung von sommertrockenen Standorten mit heimischen Baumarten in Bosnien. Dissertation, 1. Aufl. Göttingen, Cuvillier Verlag.

Vor, T., Spellmann, H., Bolte, A., Ammer, C., 2015. Potenziale und Risiken eingeführter Baumarten, in: Potenziale und Risiken eingeführter Baumarten - Baumartenportraits mit naturschutzfachlicher Bewertung, Göttinger Forstwissenschaften. Universitätsverlag Göttingen, p. 233.

VwG Köln, 2014. Verwaltungsgericht Köln: Klage zur Aufnahme der Baumart Paulwonia in die Liste der KUP-Baumarten. Privatklage und daher hier ohne Aktenzeichen angegeben.

Wagner, P., Heinrich, J., Kröber, M., Schweinle, J., Große, W., 2009. Ökonomische Bewertung von Kurzumtriebsplantagen und Einordnung der Holzerzeugung in die Anbaustruktur landwirtschaftlicher Unternehmen, in: Reeg, T., A. Bemmann, W. Konold, D. Murach und H. Spiecker (Hrsg.), Anbau und Nutzung von Bäumen auf landwirtschaftlichen Flächen, John Wiley \& Sons, Weinheim. p. 1.

Wahren, A., Schwärzel, K., Feger, K.H., Münch, A., Dittrich, I., 2007. Identification and model based assessment of the potential water retention caused by land-use changes. Advances in Geosciences 11, 49-56.

Wang, P., 1980. Economic insect fauna of China. Fasc. 21. Lepidoptera, Pyralidae. Science Press. Beijing, China, p. 229.

Wang, Q., Shogren, J.F., 1992. Characteristics of the crop-paulownia system in China. Agriculture, Ecosystems \& Environment 39, 145-152. https://doi.org/10.1016/01678809(92)90050-L

Weber, E., Broennimann, O., 2013. Invasive Pflanzen der Schweiz: erkennen und bekämpfen. Haupt, Bern.

WeGrow, 2020. WeGrow-GmbH, Düsseldorf, Kehn 20, 47918 Tönisvorst - www.wegrow.de [WWW Document]. WeGrow GmbH. URL https://www.wegrow.de/ (accessed 7.21.20).

Wermelinger, B., Wyniger, D., Forster, B., 2008. First records of an invasive bug in Europe: Halyomorpha halys Stål (Heteroptera: Pentatomidae), a new pest on woody ornamentals and fruit trees? MITTEILUNGEN DER SCHWEIZERISCHEN ENTOMOLOGISCHEN GESELLSCHAFT BULLETIN DE LA SOCIÉTÉ ENTOMOLOGIQUE SUISSE 81: $1-8,9$.

WiBei, 2008. Klimawirkungen auf Naturraum und menschliche Nutzung; Wissenschaftlicher Beirat der Bundesregierung Globale Umweltveränderungen, in: Welt im Wandel: Sicherheitsrisiko Klimawandel, Welt im Wandel. Springer, Berlin, Heidelberg, pp. 59-80. https://doi.org/10.1007/978-3-540-73248-8_6

Wilhalm, T., Stockner, W., Tratter, W., 2002. Für die Flora Südtirols neue Gefäßpflanzen (2), Ergebnisse der floristischen Kartierung vornehmlich aus den Jahren 1998-2002 2, 24.

Williams, C.E., 1993. Age structure and importance of naturalized Paulownia tomentosa in central Virginia streamside forest. [WWW Document]. URL https://www.cabi.org/isc/abstract/19960601461 (accessed 5.20.20).

Wojtkowski, P.A., 2002. Agroecological Perspectives in Agronomy, Forestry and Agroforestry. Enfield, USA: Science Publishers Inc. (2002), pp. 356. Experimental Agriculture 39, 450450. https://doi.org/10.1017/S0014479703241417

Wortmeier, A., 2017. Biomasseentwicklung von Paulownia elongata, fortunei und tomentosa bei unterschiedlichen Bewässerungsstufen, Bachelorarbeit an der Fakultät für Forstwissenschaften und Waldökologie der Georg-August-Universität Göttingen, unveröffentlicht.

Wu, H., 1995. Insects of Baishanzu Mountain, Eastern China. China Forestry Publishing House, Beijing China, p. 586.

Young, A., 1997. Agroforestry for soil management. CABI, New York, 320 S. 
Yu, P., Wang, S., Yang, X., 1996. Economic insect fauna of China. Fas. 54. Coleoptera: Chrysomelodidea (II). Science Press. Beijing, China, p. 324.

Yun, Y., 1998. Flora Fungorum Sinicorum, in: Peronosporales. Science Press. Beijing, China, p. 530.

Zehlius-Eckert, W., 2018. Bestehende rechtliche Regelungen und wünschenswerte Verbesserungen für eine verstärkte Förderung von Agroforstsystemen in Deutschland. 6. Forum Agroforstsysteme: „Brücken bilden“ - Agroforst als Bestandteil einer zukunftsgerechten und regional angepassten Landnutzung - Status quo, Bedarf und Perspektiven am 9.-10.10.2018 in Göttingen.

Zhang, S., 1995. Economic insect fauna of China. Fasc. 50 Hemiptera (II). Science Press. Beijing, China, p. 169.

Zhang, S., 1985. Economic insect fauna of China. Fasc. 31 Hemiptera (I). Science Press. Beijing, China, p. 242.

Zhao, Z., 1994. Economic insect fauna of China. Fasc. 42. Lepidoptera, Lymantriidae (II). Science Press. Beijing, China, p. 165.

Zhu, H., 1996. Fauna Sinica, Insects, Vol. 5. Lepidoptera. Bombycidae, Saturniidae, Thyrididae. Science Press. Beijing, China, p. 302.

Zhu, H., 1980. Economic insect fauna of China. Fasc. 22. Lepidoptera, Sphingidae. Science Press. Beijing, China, p. 84.

Zhu, H., Wang, L., 1997. Fauna Sinica. Insects. Vol. 11. Lepidoptera Sphingidae. Science Press. Beijing, China, p. 410.

Zhu, Z.H., Cai, M., Wang, S., Jiang, Y., 1991. Agroforestry Systems in China.

Zhu, Z.H., Chao, C.J., Chao, C.J., Xiong, Y.G., 1986. Paulownia in China: cultivation and utilization. Asian Network for Biological Sciences and International Development Research Centre, Chines Academy of Forestry Staff, Beijing.

\section{Selbständigkeitserklärung}

$\begin{array}{ll}\text { Name: } & \text { Ray Wollenzien } \\ \text { Matrikelnummer: } & 21028835\end{array}$

Ich versichere hiermit, dass ich die vorliegende Arbeit ohne fremde Hilfe selbstständig verfasst und nur die von mir angegebenen Quellen und Hilfsmittel verwendet habe. Wörtlich oder sinngemäß aus anderen Werken entnommene Stellen habe ich unter Angabe der Quellen kenntlich gemacht.

Des Weiteren versichere ich, dass von mir anderweitig keine entsprechende Promotion beantragt wurde. Auch wurde die hier vorliegende Dissertation oder Teile davon nicht woanders eingereicht.

Die Richtlinien zur Sicherung der guten wissenschaftlichen Praxis an der Universität Göttingen wurden von mir beachtet. Eine eingereichte digitale Version stimmt mit der schriftlichen Fassung überein. Mir ist bewusst, dass bei Verstoß gegen diese Grundsätze die Prüfung mit nichtbestanden bewertet wird. 


\section{Curriculum Vitae}

Persönliche Daten

Name

Ray Wollenzien

Geburtsdatum

Geburtsort

Staatsangehörigkeit

AKADEMISCHE AUSBILDUNG

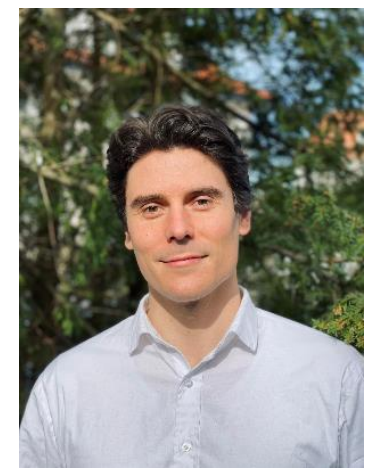

BERUfLICHE AUSBILDUNG

SCHULISCHE AUSBILDUNG \& SONSTIGES 